PFC/RR-94-12

DOE/ETf51013-307

\title{
Measurement of Particle Transport Coefficients on Alcator C-Mod
}

\author{
Thomas Cho-Tak Luke \\ Plasma Fusion Center \\ Massachusetts Institute of Technology \\ Cambridge, MA 02139
}

October 1994

\section{DISCLAIMER}

This report was prepared as an account of work sponsored by an agency of the United States Government. Neither the United States Government nor any agency thereof, nor any of their employees, makes any warranty, express or implied, or assumes any legal liability or responsibility for the accuracy, completeness, or usefulness of any information, apparatus, product, or process disclosed, or represents that its use would not infringe privately owned rights. Reference herein to any specific commercial product, process, or service by trade name, trademark, manufacturer, or otherwise does not necessarily constitute or imply its endorsement, recommendation, or favoring by the United States Government or any agency thereof. The views and opinions of authors expressed herein do not necessarily state or reflect those of the United States Government or any agency thereof.

\section{MASTER}

This work was supported by the U. S. Department of Energy Contract No. DE-AC0278ET51013. Reproduction, translation, publication, use and disposal, in whole or in part by or for the United States government is permitted. 


\section{DISCLAIMER}

Portions of this document may be illegible in electronic image products. Images are produced from the best available original document. 


\title{
MEASUREMENT OF PARTICLE TRANSPORT COEFFICIENTS ON ALCATOR C-MOD
}

\author{
by \\ Thomas Cho-Tak Luke \\ B.Sc., Physics, California Institute of Technology \\ (1986) \\ S.M., Sloan School of Management, Massachusetts Institute of Technology \\ (1994) \\ Submitted to the Department of Physics \\ in Partial Fulfillment of the Requirements of the Degree of \\ Doctor of Philosophy in Physics \\ at the \\ Massachusetts Institute of Technology \\ October 1994 \\ (C) 1994 Thomas C.T. Luke. All rights reserved.
}

The author hereby grants to MIT permission to reproduce and to distribute publicly paper and electronic copies of this thesis document in whole or in part.

Signature of Author

Department of Physics

October 27, 1994

Certified by

Dr. James H. Irby

Thesis Supervisor

Accepted by 


\title{
Measurement of Particle Transport Coefficients on Alcator C-Mod
}

\author{
by \\ Thomas Cho-Tak Luke \\ Submitted to the Department of Physics \\ on October 27, 1994 in partial fulfillment of \\ the requirements of the degree of \\ Doctor of Philosophy in Physics
}

\begin{abstract}
The study of particle transport is increasingly important for fusion research. During the 1993 and 1994 campaigns of Alcator C-Mod, one observed phenomenon was the divertor detachment. This phenomenon was characterized by reduced particle and energy flows to the divertor plates and is of interest as a solution to the heat loading problem of the first wall. A sharp rise in the central electron density was observed after detachment. The goal of this thesis was to study the behavior of the plasma transport during the divertor detachment in order to explain this density rise.

The measurement of particle transport coefficients requires sophisticated diagnostic tools. A two color interferometer system was developed and installed on Alcator C-Mod to measure the electron density with high spatial $(\approx 2 \mathrm{~cm})$ and high temporal $(\leq 1.0 \mathrm{~ms})$ resolution. The system consists of $10 \mathrm{CO}_{2}(10.6 \mu \mathrm{m})$ and $4 \mathrm{HeNe}(.6328 \mu \mathrm{m})$ chords that are used to measure the line integrated density to within $0.08 \mathrm{CO}_{2}$ degrees or $2.3 \mathrm{x}$ $10^{16} \mathrm{~m}^{-2}$ theoretically.

Using the two color interferometer, a series of gas puffing experiments were conducted. The density was varied above and below the threshold density for detachment at a constant magnetic field and plasma current. Using a gas modulation technique, the particle diffusion, D, and the convective velocity, V, were determined. Profiles were inverted using a SVD inversion and the transport coefficients were extracted with a time regression analysis and a transport simulation analysis. Results from each analysis were in good agreement.
\end{abstract}


Measured profiles of the coefficients increased with the radius and the values were consistent with measurements from other experiments. The values exceeded neoclassical predictions by a factor of 10 . The profiles also exhibited an inverse dependence with plasma density. The scaling of both attached and detached plasmas agreed well with this inverse scaling. This result and the lack of change in the energy and impurity transport indicate that there was no change in the underlying transport processes after detachment. On the contrary, measurements of the $\mathrm{H}_{\alpha}$ radiation and argon transport were consistent with a change in the electron source at the edge. Thus, the rise in central density was due to an increase in the overall electron source or a improvement in fueling efficiency and not due to an improvement in the core particle transport.

Advisor: Dr. J. H. Irby

Reader: Dr. G. Bekefi

Reader: Dr. M. Porkolab 


\section{Acknowledgments}

In the course of a thesis, many people play an important role. I would like to sincerely express my thanks and gratitude to those people that have made this thesis possible. First and foremost, I must acknowledge the efforts of Jim Irby. Without his support, patience and sound advice, this thesis would not have been possible. His support through the dark days of Alcator C-Mod made this thesis a reality. In addition, the Alcator group has been an essential part of my education. I would like to thank the many people who have helped $m e$ in the process. Their fruitful conversations and teachings have been invaluable. I'd like to thank S. Wolfe, M. Greenwald, B. Labombard, J. Goetz, M. Porkolab, S. Horne, B. Lipshultz, E. Marmar, J. Rice, R. Boivin, J. Snipes and G. Bekefi. Their support, encouragement and, of course, data have been instrumental to this thesis. I would also like to thank the other slaves. We have traveled quite an adventure together. Without P. O'Shea, M. Graf, P. Stek, A. Niemczewski, T. Hsu, C. Kurz, J. Urbann, D. Jablonski, J. Tinios, S. Tsui, D. Garnier, J. Schacter and L. Wang, no one would have carried me home from the Miracle of Science. My partners in crime have been a great source of support in the past few years. Finally, I would like to thank the many people who have supported the Alcator effort. Thanks goes to R. Murray, T. Toland, B. Childs, F. Silva, J. Nicholson, M. Iverson, R. Danforth, and S. Tambini. Your dedication and hard work has made my work easier.

Throughout the years, my friends have proven to be true and a great source of strength. I can never forget the support of Will Evans, Mike Yoo, Terry Grimm, Jim Gelb, Alex Becker, Carson Chow, Terry Hwa, Kin Cheung, Gib Gibson, and Steve Fromm. So ends the era of Somerville Tech. Special thanks goes to Steve Hsu. From our earliest days at CalTech, his support has been instrumental to where I am today. In addition, I wish to thank A. Lo, J. Wang, P. Vasan, Z. Amrolia, G. Sharp, C. Pandolfino, M. Sullivan, and J. Sia. They have taken the pressure off me in the past few weeks and I am eternally grateful. Also I would like to thank M. Brennan for his encouragement and proofreading. It has been truly inspirational.

Finally, I would like to thank my family. They have supported and encouraged me with infinite patience. My parents, Brian and May Luke taught me to persevere and reach my full potential. They said it would be worth it and they were right. I'd like to thank my siblings, Anthony and Brigid. They have been there when I needed it. I would also like to thank Jack and "Mother" Tsui. They didn't know what they were getting in to when they let me marry their daughter but their support has been never-ending. Most of all, I would like to acknowledge with love and gratitude the patience and support of my wife, Amy. In the annals of human endeavor, no one has gone through so much for so little and I will be forever grateful.

This is the end of a long journey starting at CalTech and many people have made it a success. If I have forgotten anyone, let it be known now that I am grateful for their help. 
With love and gratitude, I dedicate this thesis to my wife, Amy. 


\section{Table of Contents}

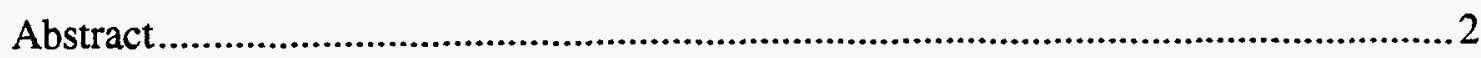

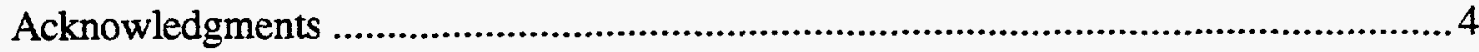

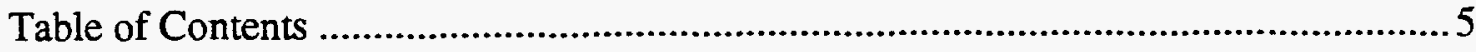

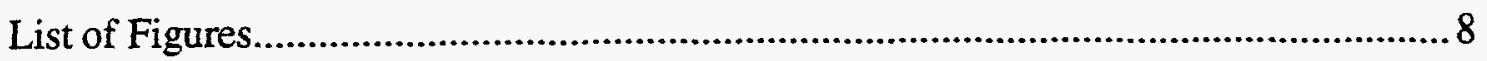

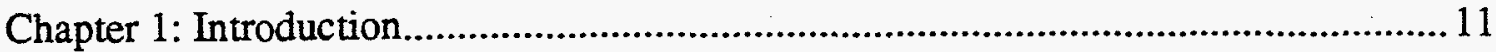

1.1 The Transport Problem ........................................................................................... 11

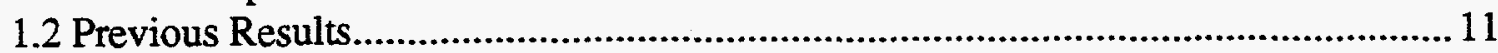

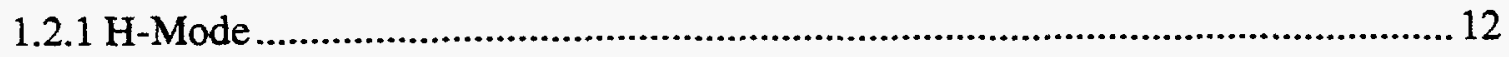

1.2.2 Peaked Confinement Modes ................................................................................ 14

1.2.2.1 Pellet Mode

1.2.2.2 Improved Ohmic Confinement (IOC) ............................................................ 14

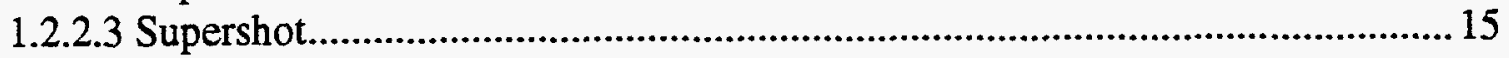

1.3 Transport Studies for Alcator C-Mod................................................................... 16

1.3.1 Detached Divertor................................................................................................16

1.3.2 Density Effects Due to Detachment.........................................................................17

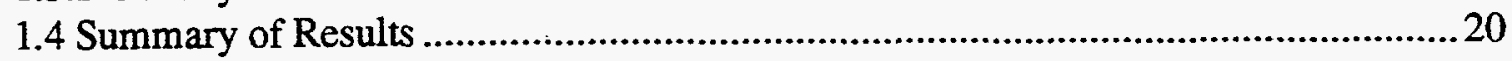

1.5 Thesis Outline

Chapter 2: Alcator C-Mod ...................................................................................25

2.1 Alcator C-Mod: Specifications and Mechanical Features ........................................25

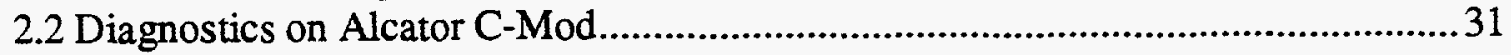

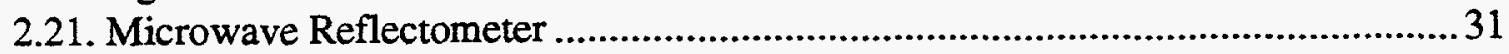

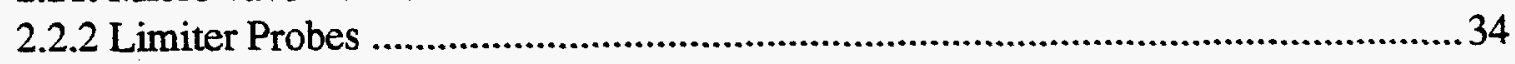

2.2.3 Fast Scanning or Reciprocating Probe ……............................................................. 34

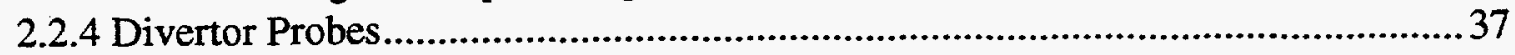

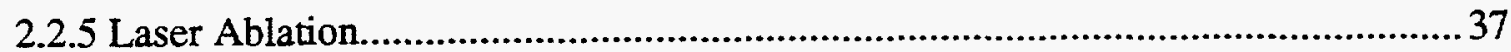

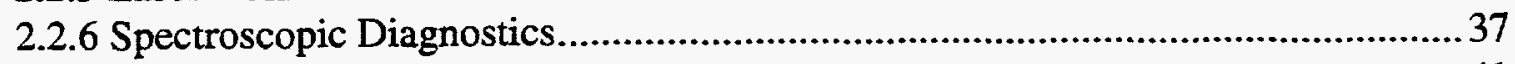

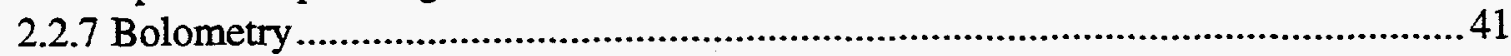

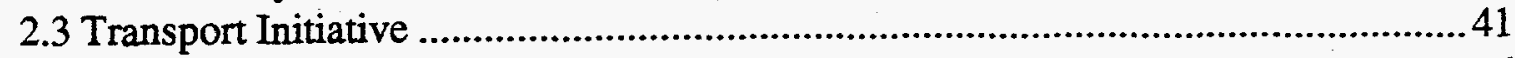

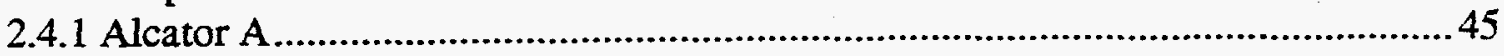

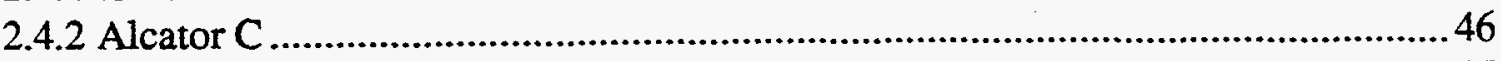

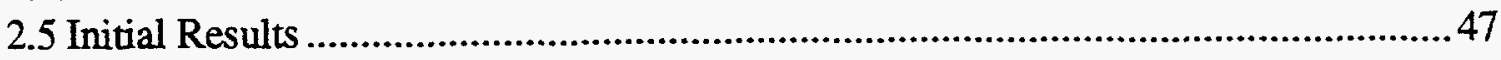

Chapter 3: Two Color Interferometer System............................................................51

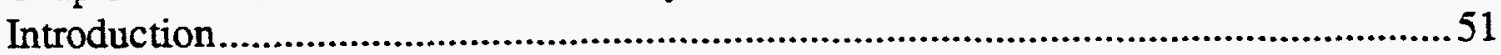

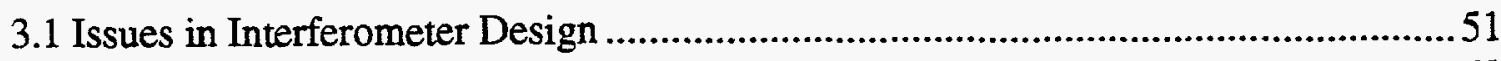

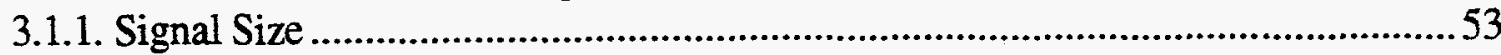

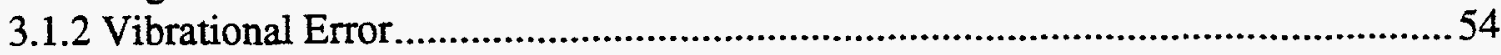




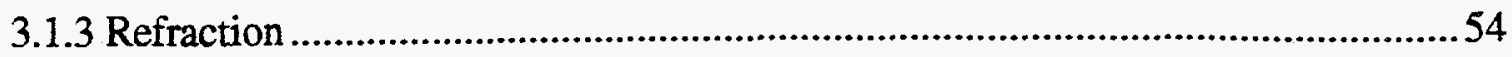

3.2 Design of the Two Color Interferometer for Alcator C-Mod ...................................56

3.2.1 Vibrational Compensation ...................................................................................56

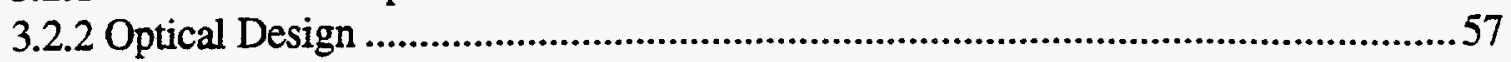

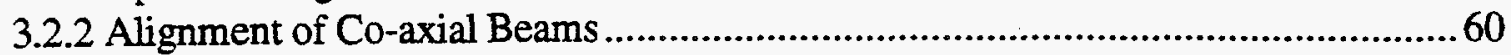

3.2.3 Plasma Beam Design .....................................................................................62 62

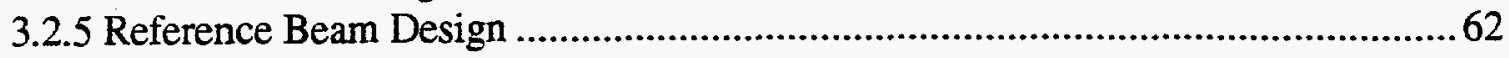

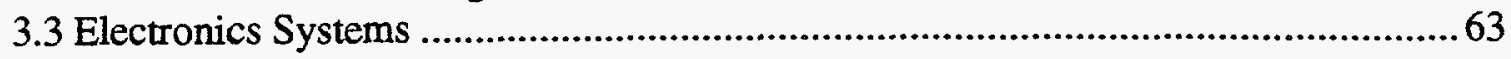

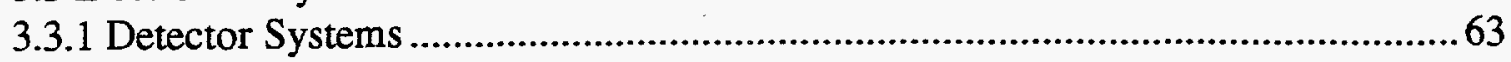

3.3.2 Phase Demodulators.....................................................................................65

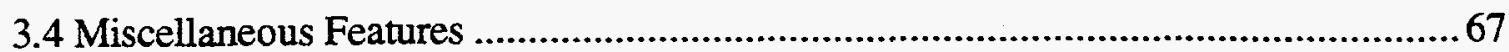

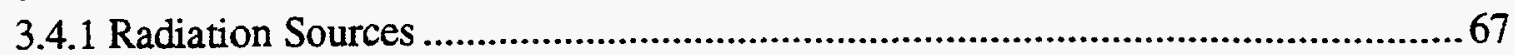

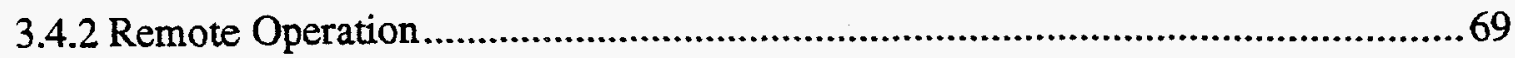

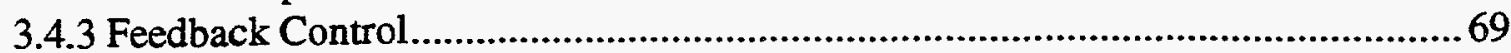

3.4.4 Acoustic and Thermal Insulation ...................................................................... 70

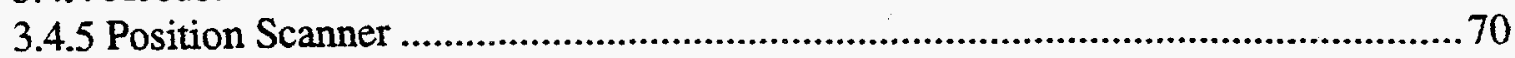

Chapter 4: Profile Inversions ................................................................................. 71

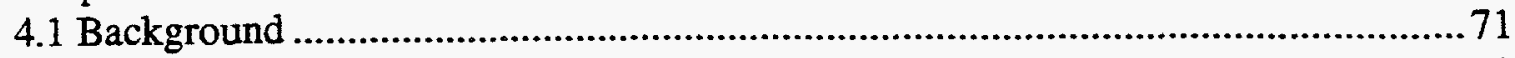

4.2 Inversion Analysis for Alcator C-Mod ...................................................................

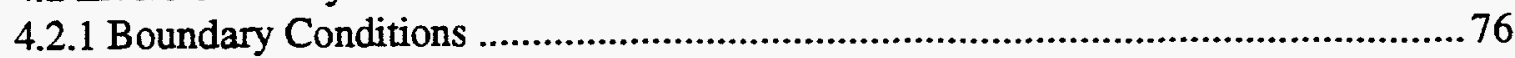

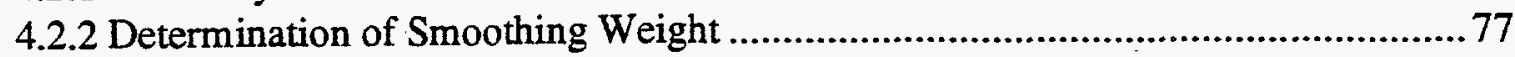

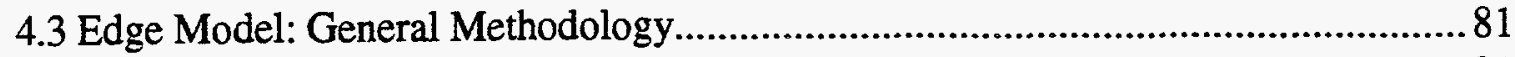

4.3.1 Scalings from Fast Scanning Probes. ................................................................... 81

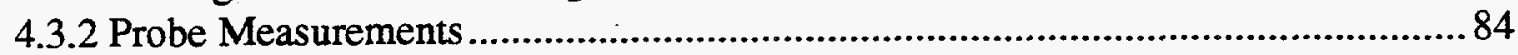

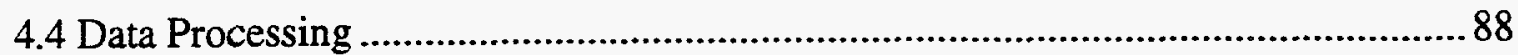

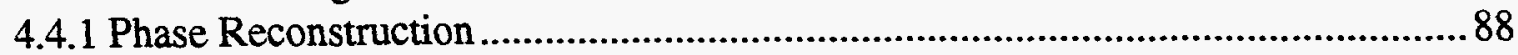

4.5 Experimental Checks of the Inversion................................................................... 89

Chapter 5: Transport Analysis ................................................................................97

5.1 Framework for Transport Analysis ........................................................................97

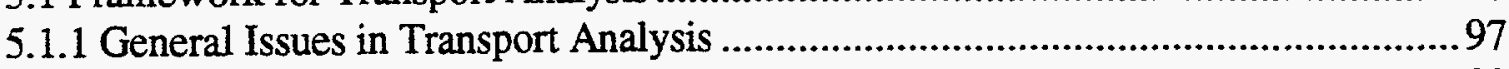

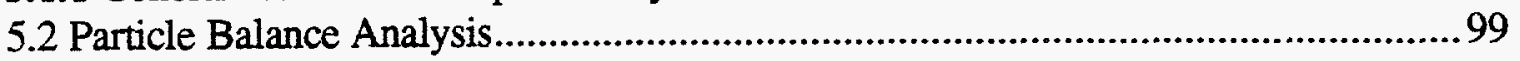

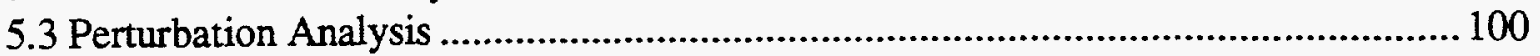

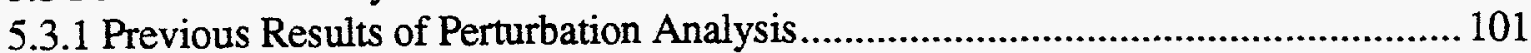

5.4 Effects of Non-linearity of the Diffusion Coefficients.............................................. 103

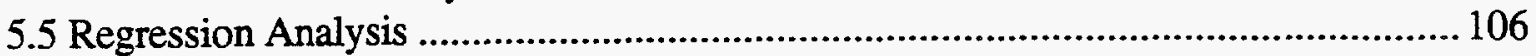

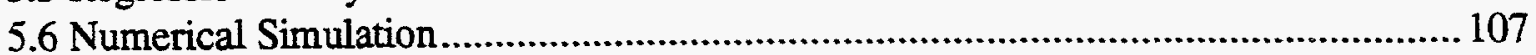

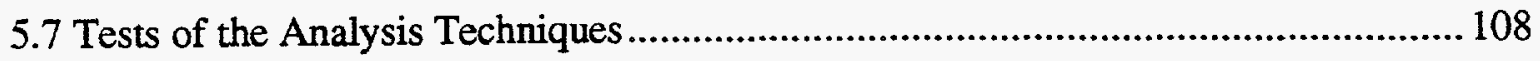

Chapter 6: Experimental Observations ....................................................................112

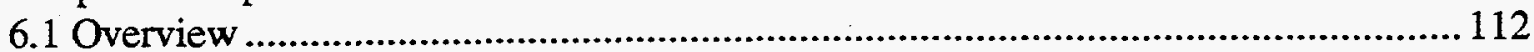

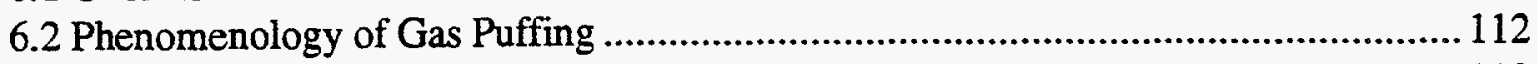

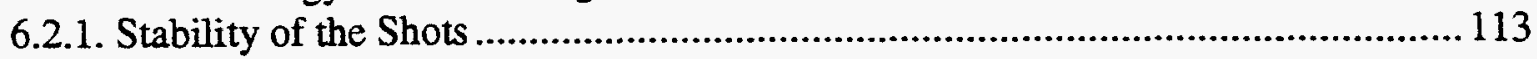

6.2.2 Profile Inversions ...........................................................................................116 
6.2.3 Results of Analysis to Determine Smoothing Weight .............................................116

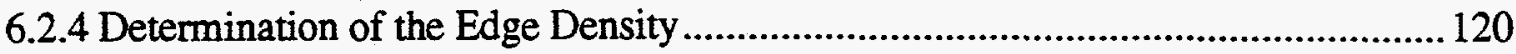

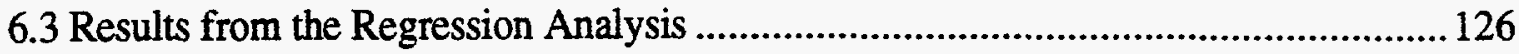

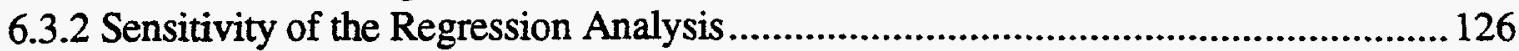

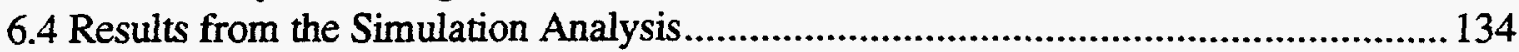

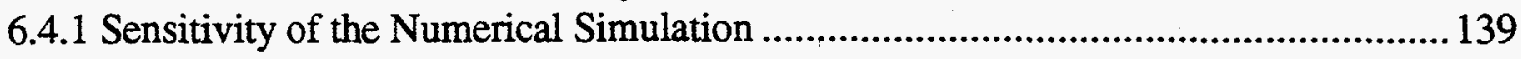

Chapter 7: Interpretation of Results .......................................................................... 143

7.1 Interpretation of Core Particle Measurements on C-Mod......................................... 143

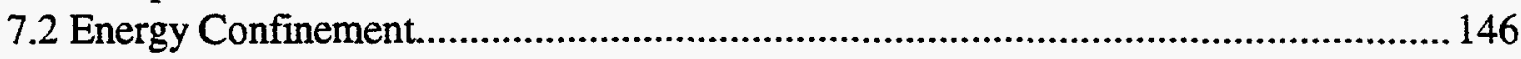

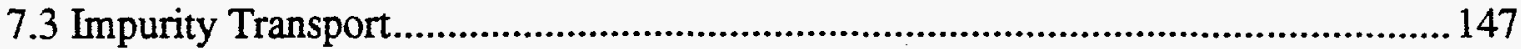

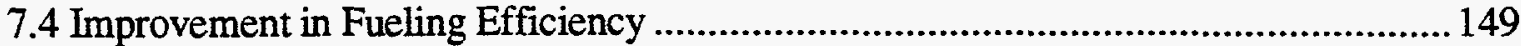

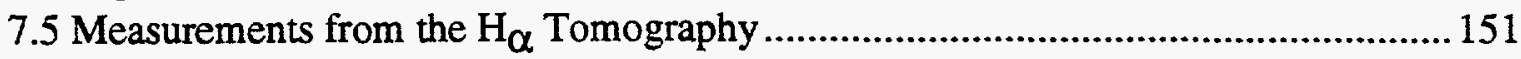

7.6 Hypothesis for the Rise in Central Density After Detachment ................................ 155

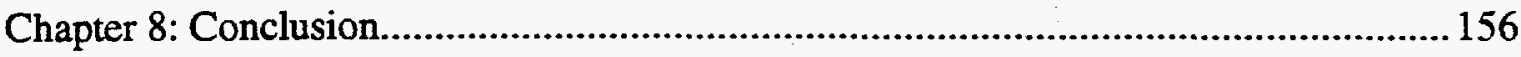

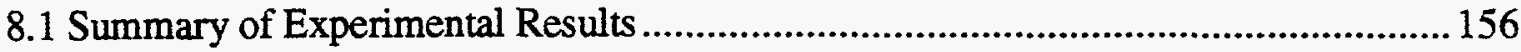

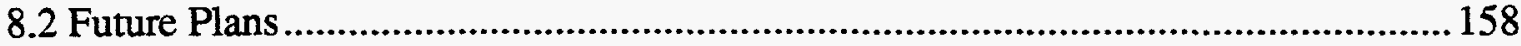

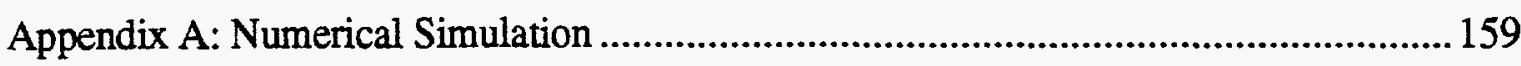

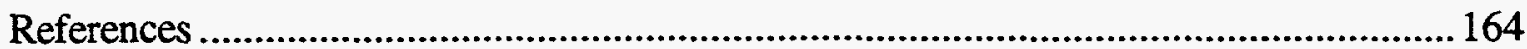




\section{List of Figures}

1.1: Ion saturation currents from the outer divertor Probes [89] ..................................18

1.2: Measured plasma parameters during detachment. Note the rise in line integrated density, $\mathrm{H}_{\alpha}$, and radiated power. Detachment occurs at $800 \mathrm{~ms}$.

1.3: Comparison of transport coefficient profiles from both the regression analysis and the numerical simulation for a range of background densities.

1.4: Scaling of transport coefficients at $\Psi=0.5$ with the inverse density.

2.1: 3-D schematic of Alcator C-Mod with cross sectional view of the ports and vacuum vessel.

2.2: Cross Sectional view of Alcator C-Mod at the midplane.........................................28

2.3: Vertical cross section of Alcator C-Mod showing the location of major system components.

2.4: Cross sectional view of Alcator C-Mod showing the location of the

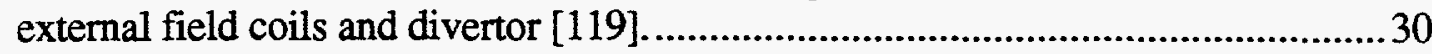

2.5: Location of the major diagnostics on Alcator C-Mod [117].................................32

2.6: Schematic of Reflectometer system [59].................................................................33

2.7: Front and side views of the RF limiters [118] ........................................................35

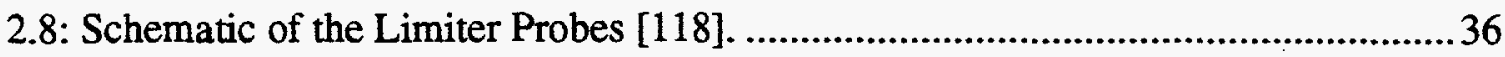

2.9: Location of reciprocating probe and divertor probes in the divertor

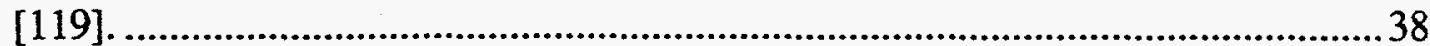

2.10: The four probe head of the reciprocating probe [119].............................................

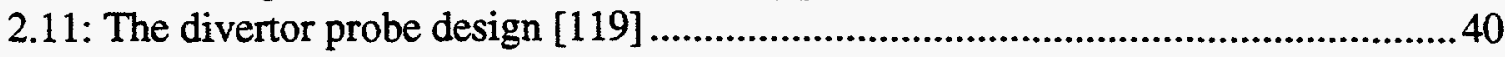

2.12: Layout of the view for the VUV Spectrometer [62]...............................................42

2.13: Location of the toroidally viewing bolometer array and corresponding

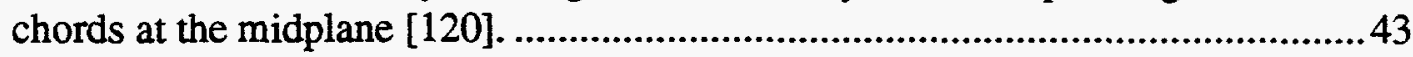

2.14: Location of the divertor bolometer arrays [120] ...............................................44

2.15: Comparison of the measured energy confinement time on Alcator CMod during the 1993/1994 campaign with L-Mode and Neo-Alcator

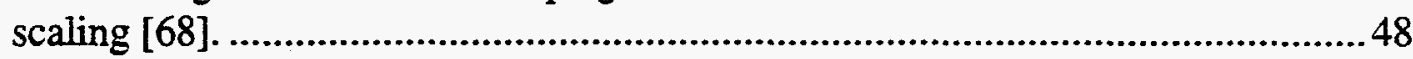

2.16: Comparison of H-Mode threshold on Alcator C-Mod with the power scaling from ASDEX-Upgrade and DIII-D [68]................................................50

3.1: Location of TCI chords relative to the plasma................................................52

3.2: Raytracing results for a simulated pellet injection using 10.6 and $119 \mu \mathrm{m}$ wavelengths. Shifts in the chord location due to refraction are listed under

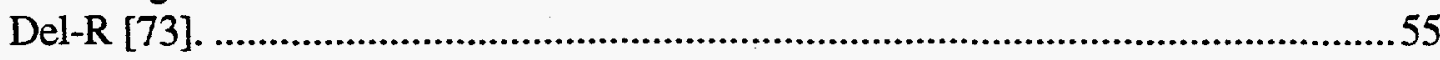

3.3: Optical layout for the TCI. .................................................................................5

3.4: Results of geometric raytracing of the beam through the plasma and reference

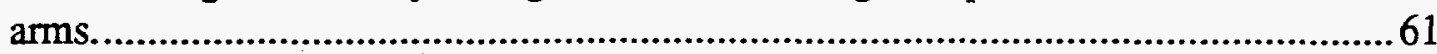

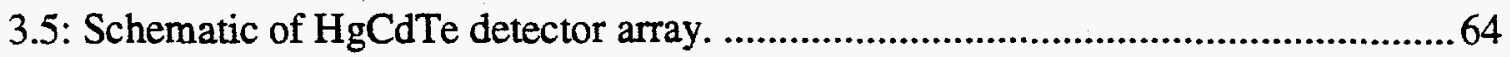

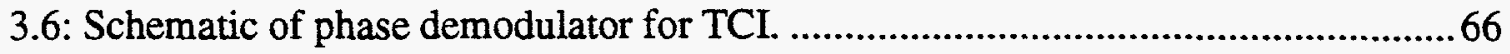


3.7: Location of TCI system relative to the vacuum vessel. 68

4.1: Comparison of $n_{e}$ and $T_{e}$ measurements from the divertor probes and reciprocating probe. .73

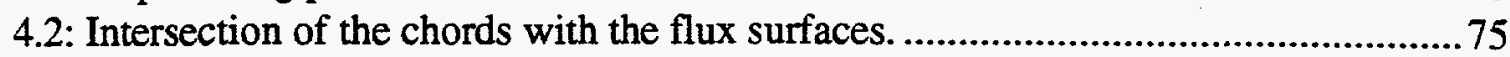

4.3: Typical result from the epsilon analysis. Error in the edge density must be $<0.1$ and the chisq error must be $<0.05$.

4.4: Measurement of the density profile in the scrapeoff layer from the reciprocating probe. 82

4.5: Scaling of the edge density and the scrapeoff length with central density and plasma current using the reciprocating probe.

4.6: Comparison of the particle inventory as calculated from the inversion using two edge densities.

4.7: Comparison of the inverted and initial density profiles for a) a parabolic profile

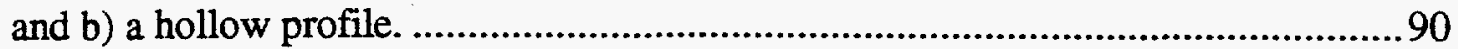

4.8: Inversion of double pellet shot. ............................................................................

4.9: Effect of shifts in the magnetic geometry on the inverted profile............................93

4.10: Effect of the smoothing weight, $\varepsilon$, on the inverted profile....................................94

4.11: Effect of the edge density on the inverted profile..............................................95

4.12: Effect of the edge gradient on the inverted profile. .................................................96

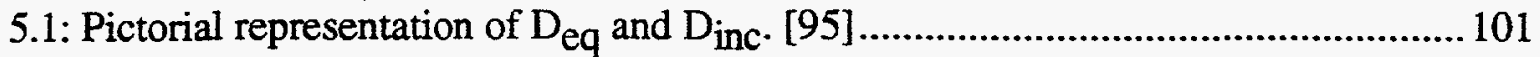

5.2: Comparison of coefficient profile as a function of $\mathrm{C} 0$............................................. 109

5.3: Comparison of simulated coefficient profile with regressed profiles using $0 \%$ and $1 \%$ noise.

6.1: Interferometer signals on chords $1,3,4,5$, and 6 during gas puff. .....................114

6.2: Interferometer signals for repeated gas puffs. ..................................................115

6.3: Time histories of plasma parameters during gas modulation. All parameters exhibit good stability during the puffing.

6.4: Standard outputs of inversion analysis: particle inventory, peak density, line averaged and peaking factor.

6.5: Time evolution of density profile during a gas puff.

6.6: Errors from epsilon analysis for inversions with different edge densities: a)0.1, b) 0.2, c) 0.3 , d) 0.4 , and e) 0.5 .

6.7: Comparison of predicted and measured reflectometer signals for the best estimate of the edge density from the epsilon analysis.

6.8: Comparison of predicted and measured reflectometer signals for the 2 times the best estimate of the edge density from the epsilon analysis.

6.9: Transport coefficient profiles from regression

6.10: Comparison of transport coefficient profiles for line averaged densities of $7.4 \times 1019$ to $1.31 \times 1020 \mathrm{~m}-2$.

6.11: Variation of regressed transport coefficients with edge density.............................130

6.12: Variation of regressed V/D ratio with edge density...

6.13: Variation of transport coefficients profiles with smoothing weight, $\varepsilon$. 132

6.14: Comparison of reconstructed time derivative of the particle inventory from 
the regression and the measured time derivative.

6.15: Comparison of regressed coefficient profiles for regressions over three puffs individually and over all three puffs simultaneously.

6.16: Variation of coefficient profiles with shifts in the magnetic geometry.

6.17: Comparison of transport coefficient profiles from the regression analysis and the numerical simulation.

6.18: Comparison of transport coefficient profiles from the regression analysis and the numerical simulation for the same background densities as fig. 6.9 .

6.19: Comparison of simulated and measured particle inventory time histories............. 140

6.20: Comparison of the measured and simulated time histories of the interferometer signals.

6.21: Variation of the simulated phase signals when the transport coefficients are varied by $\pm 10 \%$ while maintaining the V/D ratio.

7.1 Scaling of transport coefficients at $\Psi=0.5$ with the inverse density.

7.2: Time history of the brightness of the Scandium line emission as

measured by xrays and VUV diagnostics.

7.3: Rise in the central Argon density after detachment.

7.4: Spatial distribution of the $\mathrm{H}_{\alpha}$ radiation before detachment at $800 \mathrm{~ms}$.

7.5: Spatial distribution of the $\mathrm{H}_{\alpha}$ radiation after detachment at $800 \mathrm{~ms}$.

The source increases and moves inside the LCFS.

7.6: Number of ionizations inside the LCFS. Detachment occurs at $800 \mathrm{~ms}$.

A.1: Sensitivity of simulated transport coefficient profiles with source width. 162

A.2: Neutral density profile from FRANTIC code. 


\section{Chapter 1}

\section{Introduction}

\subsection{The Transport Problem}

As it has been from the very beginning of fusion research, the problem of particle and energy confinement remains an active area of interest [1]. As experimental machines reach reactor relevant densities and temperatures, a clear understanding of the underlying transport process is crucial. The ability to control not only the central density but also the profile shape itself will be a necessity for a feasible fusion reactor. The density problem is twofold. The electron and corresponding ion density must be maintained and confined so that the number and energy of collisions is sufficient for fusion reactions. However, at the same time, the impurity density must be controlled to prevent their buildup in the core and the resulting radiation of energy. Also, the issue of alpha particle transport must be solved to prevent dilution of the fuel. The answer to these questions represents an underlying goal for the fusion program and provides the motivation for transport studies in order to gain a deeper understanding of different transport phenomena.

\subsection{Previous Results}

The early description of transport in tokamaks relied on classical and neo-classical theory $[2,3]$. However, experimental observations have proven both theories to be inadequate in explaining the anomalously large fluxes. Electron energy and particles fluxes have been found to be up to a factor of 100 greater than neoclassical predictions [4]. Typically, only current diffusion and, in special cases, impurity/ion transport can be explained by neoclassical theories $[5,6]$. As a result, most transport models rely on 
empirical scalings in order to make predictions. Goldston and Neo-Alcator confinement scalings are representative of this type of result $[7,8]$.

In addition to the development of scalings laws, recent experiments have discovered new confinement regimes in which the particle and energy confinement exceeds that predicted by either theory or empirical scalings. L-Mode scaling has been theorized to be a manifestation of several underlying transport mechanisms and a lower bound for confinement [9]. When any of these mechanisms are suppressed, an enhanced confinement mode is entered. The most famous of these is the H-Mode which was first discovered on ASDEX [10]. Similarly other modes such as the Improved Ohmic Confinement ( IOC) [11, 12], Pellet Enhanced Performance (PEP) [13,14] and Supershot on TFTR [15, 17] have been found. These enhanced modes can be broken into two categories: 1) those due to changes in the edge plasma (e.g. H-Mode), and 2) those that involve a peaking of the density profile ( e.g., IOC, PEP, Supershot): One possible explanation for the enhancement of confinement in the latter category is the suppression of ion temperature gradient (ITG) or $\eta_{\mathrm{i}}$ modes due to the steepening of the edge gradients $[18,19,20]$. The study of these improved confinement regimes offers an opportunity for a better understanding of the underlying causes of anomalous transport. The following two sections will review the phenomenology of enhanced confinement modes.

\subsubsection{H-Mode}

First observed at ASDEX, the H-mode is a clearly observed transition from Lmode in which particle confinement improves as evidenced by reduced $H_{\alpha}$ or $D_{\alpha}$ emission, increased density confinement, and increased energy confinement by up to a factor of 3 over L-mode [10]. There exists a threshold in heating power above which a plasma can enter the $\mathrm{H}$-mode. This phenomenon has been observed in both diverted and limited plasmas with the $\mathrm{H}$-mode threshold increasing for limiter operation [16]. Various 
scalings have also shown an increasing threshold with the toroidal field, $\mathrm{B}_{\mathrm{T}}$, no dependence with the plasma current, $\mathrm{I}_{\mathrm{p}}$, and an ambiguous correlation with electron density, $\mathrm{n}_{\mathrm{e}}$. Edge effects play a crucial role in H-modes. A reduction in recycling of the particles at the edge is a necessity in achieving the transition. The recycling cools the edge of the plasma and prevents the high edge temperature necessary for H-mode. Experiments on PDX [21] and ASDEX [22] have shown that decreasing the conductance paths from the main vacuum chamber to the divertor improved the H-mode considerably. Also, sufficient power flow to the walls is required for H-mode. Heating of the main plasma can be accomplished by neutral beams [10,23, 24, 25, 26], ICRF [28, 29], and even by ohmic power [29].

An edge localized mode or ELM has been observed during H-Mode [30, 31, 32]. These fast disruptions at the edge are characterized by spikes in the $\mathrm{H}_{\alpha}$ light and the observation of fast particle and energy transport into the scrape off layer. Impurities flow inward when the electron density is peaked immediately after the ELM. As the electron density relaxes and flattens between ELMs the impurities reverse direction and flow to the edge. The activation of the next ELM then expels the contaminants from the plasma. This results in an effective control mechanism for cleansing the plasma of impurities since impurity recycling is low $[30,31]$.

Real improvement in confinement is also observed. Transport is greatly reduced and measured diffusion coefficients are very small as the edge gradients peak up sharply in $\mathrm{H}$-mode. Edge fluctuation measurements observe a decrease in turbulence levels [34]. Experiments on ASDEX [36], JET [37] and PDX [35] have demonstrated improvement in both core transport as well as at the edge. In fact, observations on ASDEX indicate that the H-mode originates inside the LCFS and the effects in the edge plasma are a response to the changes in the power and particle fluxes across the separatrix [38]. 


\subsubsection{Peaked Confinement Modes}

In addition to the $\mathrm{H}$-mode, other enhanced confinement modes have been observed. The most notable feature of these modes is the peaking of the density profiles during improved confinement. Typically, it has been thought that the increased gradients at the edge suppress the $\eta_{\mathrm{i}}$ modes and reduce transport. These modes can be initiated by either pellet injection (Alcator C, JET), NBI heating or systematic gas puffing.

\subsubsection{Pellet Mode}

The pellet mode was first seen in Ohmic experiments on Alcator C. Later studies showed that this phenomena occurs for ICRF or neutral beam heated plasmas as well [ 41, 42 43]. It was found that pellet injection allowed the circumvention of the saturation in energy and particle confinement that resulted from gas fueling. The observed improvement in energy confinement is consistent with the ITG model in which $\chi_{i}$ is reduced to neoclassical levels [40]. Experiments at JET show peaking of both core $T_{i}$ and $\mathrm{T}_{\mathrm{e}}$ profiles indicating a possible improvement in $\chi_{e}$ also. At the same time, particle confinement is improved. The ratio of the diffusion coefficients, V/D, increases although individually $\mathrm{V}$ and $\mathrm{D}$ decrease after injection. Studies on TEXT have shown the impurity transport to be consistent with neoclassical theory [44]. Further support of the ITG model is seen in the reduction or suppression of density fluctuations in TEXT following pellet injection $[45,46]$. Thus, experimental evidence points to a reduction in the ion channel as the profiles peak resulting in improved confinement for both particles and energy. Furthermore, in the pellet mode the peaking is due to direct fueling of the core as opposed to a change in the edge conditions as will be seen in the following two modes.

\subsubsection{Improved Ohmic Confinement (IOC)}

Peaked profiles due to rapid puffing have also exhibited improved confinement [11 12]. Particle confinement improved as the ratio of the transport coefficients, V/D, 
increased. $\mathrm{D}$ was reduced by a factor of 10 and $\mathrm{V}$ by a factor of 3 . Impurity transport was also reduced by a factor of 3 . Again, a reduction of $\chi_{\mathrm{i}}$ to neoclassical levels was responsible for the improvement in confinement. The triggering mechanism of this mode is related to the edge density. If the edge neutral density was high due to low fueling efficiency, the transport processes were altered so that confinement was poor. However, when the fueling was adjusted to maintain a low edge neutral density, the V/D ratio increased and resulted in more peaked profiles. In turn, the larger edge gradients resulted in the suppression of the ITG modes and led to improved confinement [47].

\subsubsection{Supershot}

On TFTR, high power neutral beam injection has produced a peaked density mode known as the Supershot. Similar to the IOC mode, the energy confinement and peaking factor were strongly correlated to the edge density and the recycling rate. Studies have shown that when the edge density is increased, density profiles broaden and energy confinement remains at L-Mode levels [15]. A low edge density was a necessity for the supershot. At TFTR, this had been achieved by altering the recycling efficiency by preconditioning of the walls so that enhanced wall pumping was achieved. In the Supershot, peaking factors of 3 have been achieved, with a similar factor of 3 improvement in confinement. L-mode scaling was violated as the Supershot exhibited weak current and power scaling. Energy confinement improved by increasing the stored energy of both the bulk plasma and the fast ion tail. This resulted in equal increases in both total energy and thermal energy confinement time. Measurements of the transport showed that $\chi_{\mathrm{e}}$ decreased by at least a factor of two at $a / 2$ and that $\chi_{i}$ decreased by a factor of 5-8 [ 48 , 50, 51]. These reductions were well correlated with increased density peaking [49]. 


\subsection{Transport Studies for Alcator C-Mod}

Enhanced confinement modes offer a unique opportunity to study the underlying transport mechanisms. In all of the modes, one of the most important consequences is the improved particle confinement. Improving and maintaining peaked profiles is directly reactor relevant since the central fusion parameter, $\langle\mathrm{nT} \tau\rangle$, is dependent on the peaking factor of the density [47]. Thus, improvement in both the magnitude and the shape of the density profile is crucial to the future of fusion. The discovery and study of any new enhanced confinement modes would be extremely beneficial to the overall understanding of transport.

\subsubsection{Detached Divertor}

During the 1993 and 1994 campaigns of Alcator C-Mod, one of the frequently observed phenomena was the divertor detachment. This was a relatively new effect and studies have been done on only a few tokamaks $[52,53]$. The phenomena can be characterized by a sharp drop in the energy and particle fluxes to the divertor surfaces. The flows out of the last closed flux surface, LCFS, and those to the divertor tiles have become "detached" from one another.

The divertor, a specific region in the upper or lower half of the vacuum vessel to which the magnetic field lines are deliberately diverted, was originally developed to help solve the problem of impurity recycling and power dissipation at the first wall. The divertor was designed to disperse the heat flux on the first surface. Also, in a closed divertor configuration (see fig. 2.4), the conductance from the divertor back to the main plasma decreases so that impurities swept into or created in the divertor can not recycle back into the plasma. For ITER, the parallel heat flow in the scrapeoff layer is expected to be $500-1000 \mathrm{MW} / \mathrm{m}^{2}$. The heat load to the wall, however, should not exceed $5 \mathrm{MW} / \mathrm{m}^{2}$. Thus, any technique that can help dissipate the power in the scrapeoff layer is valuable. Divertor detachment on Alcator C-Mod has resulted in a drop of up to a factor of 20 in 
the power to the divertor plates [54]. Thus, detachment is a topic of interest because it supports the concept of a radiative divertor as a solution to the heat loading problem of the first wall.

This phenomena is induced by strong gas puffing at either the midplane or in the divertor and is characterized by a drop in the ion saturation currents measured by the divertor Langmuir probes [55]. Figure 1.1 shows the ion saturation current signal on the langmuir probes located at different parts of the divertor nose. Detachment occurs at 715 ms. The probes located in the closed divertor show a sudden drop in the current collected indicating a decrease in the particle and energy flux to the divertor tiles. The electron temperature at the target plates is observed to be low, $\mathrm{T}_{\mathrm{e}}<5 \mathrm{eV}$. Measurements by the fast scanning probe (FSP) of the density and temperature profiles in the scrapeoff layer indicate that the profiles at the edge broaden after detachment. Pressure along the field lines, which is conserved in normal operation, may no longer be a constant. Furthermore, there is an increase in the radiation from impurities during the detachment and the location of this emission moves closer towards the $\mathrm{x}$-point.

\subsubsection{Density Effects Due to Detachment}

The most interesting feature from the point of view of density measurements, is a dramatic rise in the line integrated signals on all the interferometer chords. Figure 1.2 shows a detachment at $800 \mathrm{~ms}$. A clear break in the slope of the interferometer signal is seen. The line averaged densities can increase by up to $50 \%$ after detachment.

Simultaneously, there is a rise in the $\mathrm{H}_{\alpha}$ and bolometer signals and the characteristic drop in the divertor probe signals [60]. This raises a clear question. Is the rise in the central density a consequence of changing edge conditions or is it the result of a change in the underlying transport? Could divertor detachment be an enhanced confinement mode? Although there is a rise in the total $\mathrm{H}_{\alpha}$ emission which would indicate an increasing source term for the core, this may be due to increases in radiation from the 


\section{Outer Divertor Probe Currents vs. Time}

Divertor Detachment occurs at $.715 \mathrm{sec}$

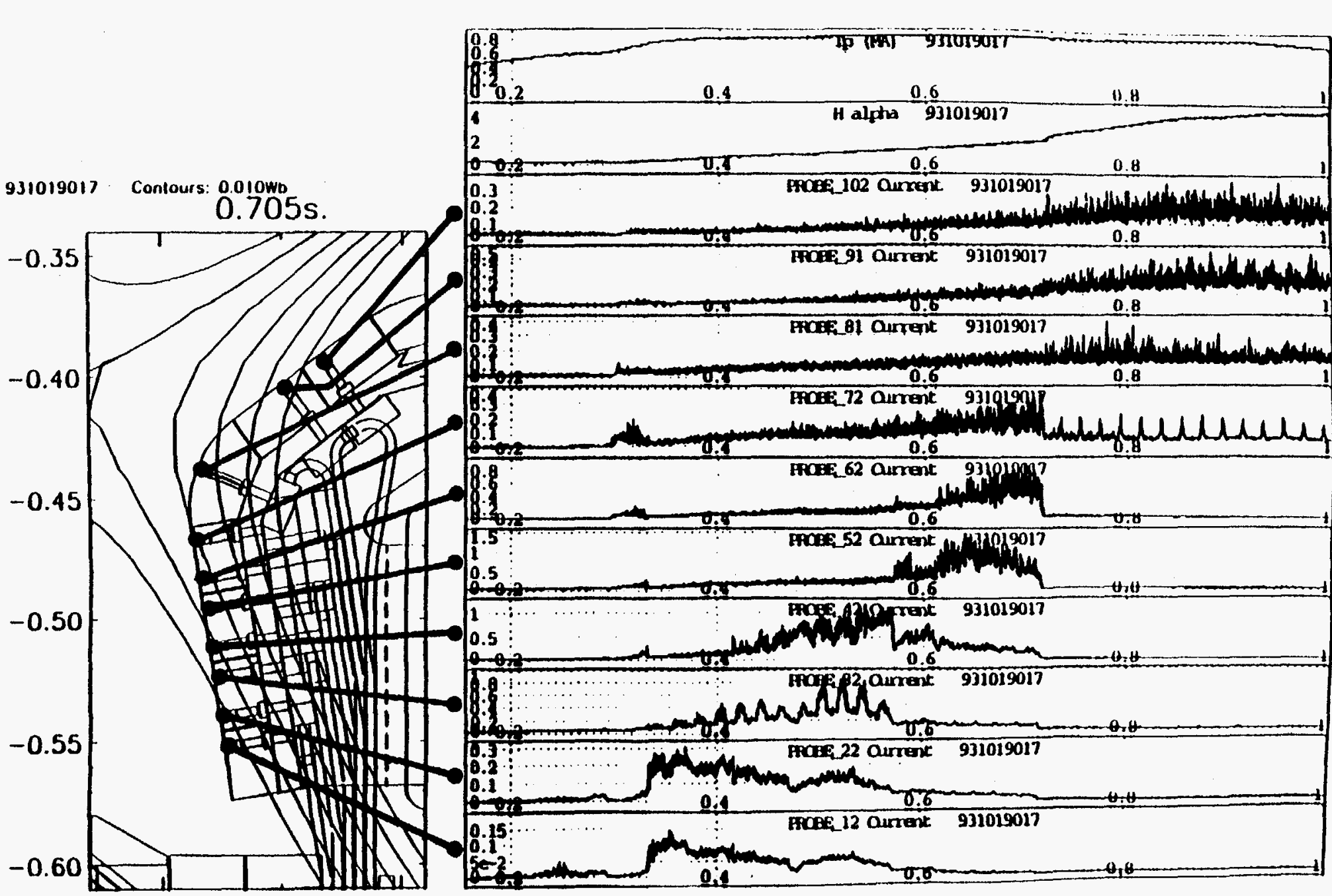

0.6

0.7 


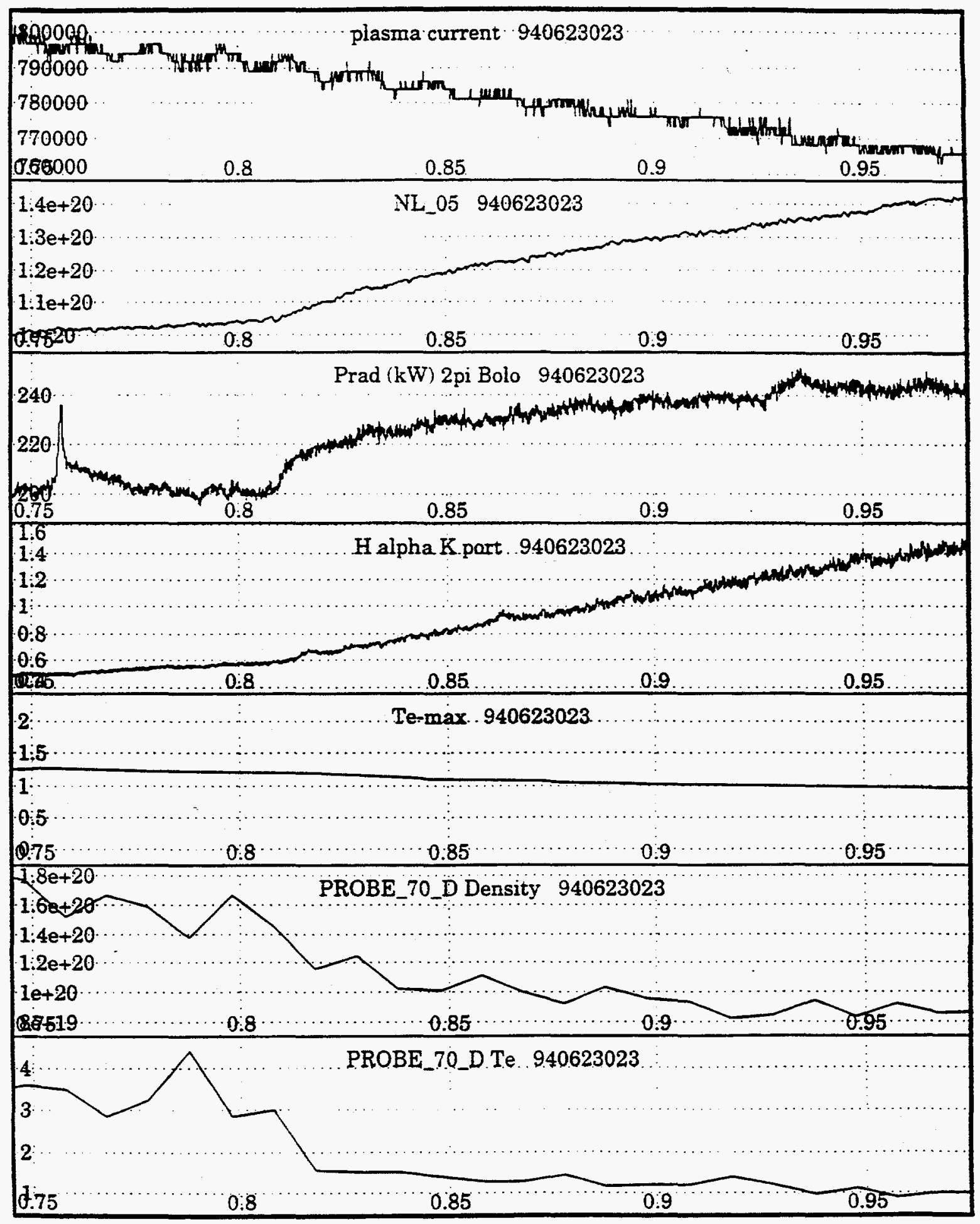

Figure 1.2: Measured plasma parameters during detachment. Note the rise in line integrated density, $\mathrm{H}_{\alpha}$, and radiated power. Detachment occurs at $800 \mathrm{~ms}$. 
closed divertor region. Most of the electrons created in the divertor would not get into the plasma and the effect of increasing the number of neutral ionizations in the divertor on core confinement is uncertain. Therefore, the true change in the electron source cannot be inferred from just the total $\mathrm{H}_{\alpha}$ emission. It is necessary to investigate the actual transport occurring in the plasma core during detachment to explain the density effects.

\subsection{Summary of Results}

In order to answer to the above mentioned question, this thesis will focus on a series of gas modulation experiments that were performed at densities near the threshold density for detachment. Gas modulation techniques are well established as a valuable method to measure the transport coefficients by creating small perturbations of the plasma. The resulting changes in the density were measured by a vertically viewing Two Color Interferometer with $10 \mathrm{CO}_{2}(10.6 \mu \mathrm{m})$ and $4 \mathrm{HeNe}(.6328 \mu \mathrm{m})$ chords. The experiment was done in a series of shots at a constant magnetic field of 5.3 Tesla and a plasma current of $630 \mathrm{kA}$. The density was varied between $7.0 \times 10^{19}-1.4 \times 10^{20} \mathrm{~m}^{-3}$ with half the shots being attached and half detached. The line integrated measurements were inverted using a SVD inversion methodology and the transport coefficients were extracted by two separate analysis techniques: a time regression analysis and a numerical simulation analysis. Both analyses gave comparable results as can be seen in figure 1.3.

The measured profiles increased in radius with values a factor of 10 greater than neoclassical values. The most significant result of the experiment was an inverse scaling of the coefficients with plasma density. In figure 1.4 , the coefficients at roughly half the minor radius are plotted as an inverse function of density. Both the attached and detached results followed the same scaling. This result was consistent with a hypothesis that there is no change in the fundamental transport when the plasma detaches. Instead the rise in 

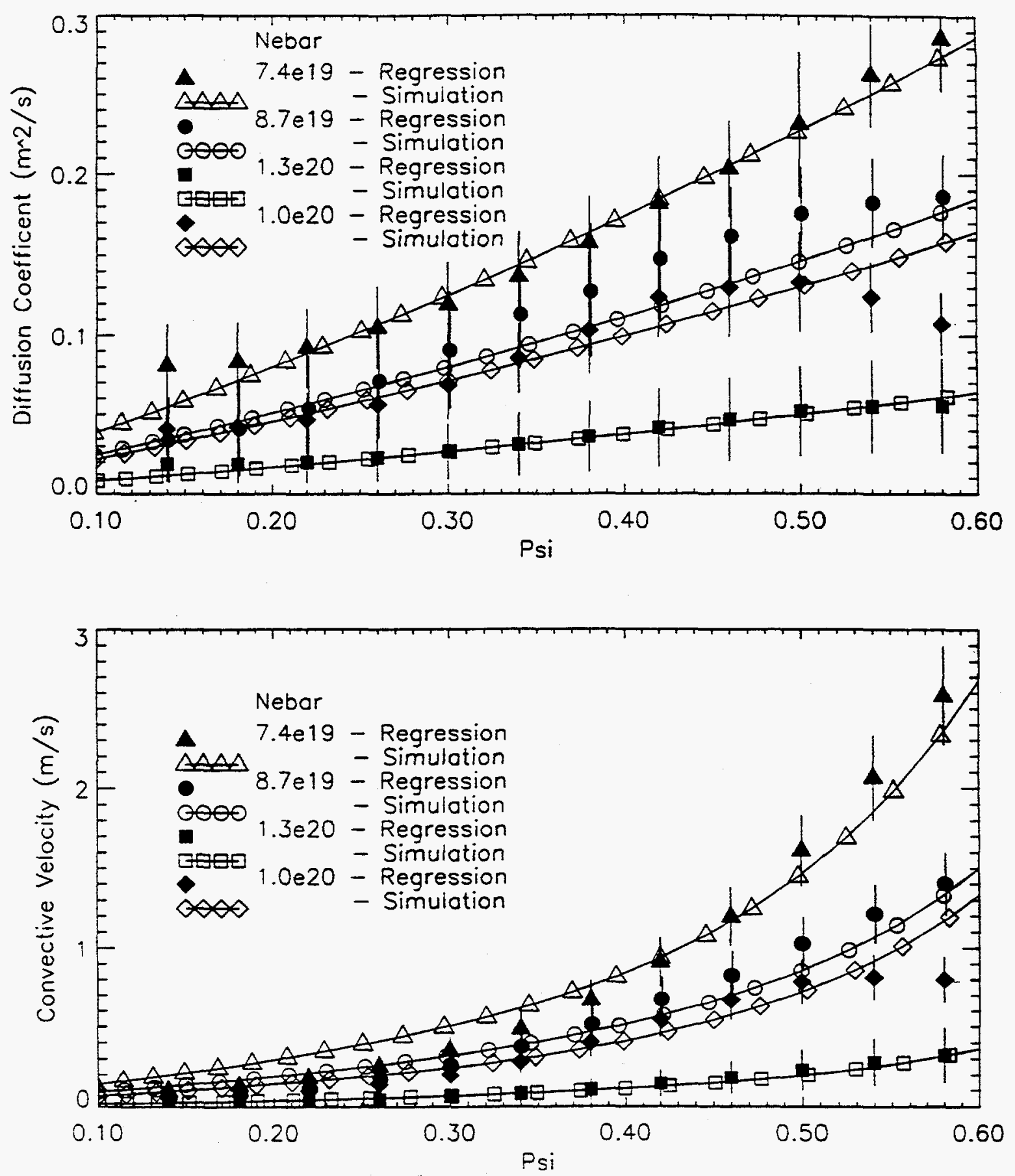

Figure 1.3: Comparison of transport coefficient profiles from both the regression analysis and the numerical simulation for a range of background densities. 

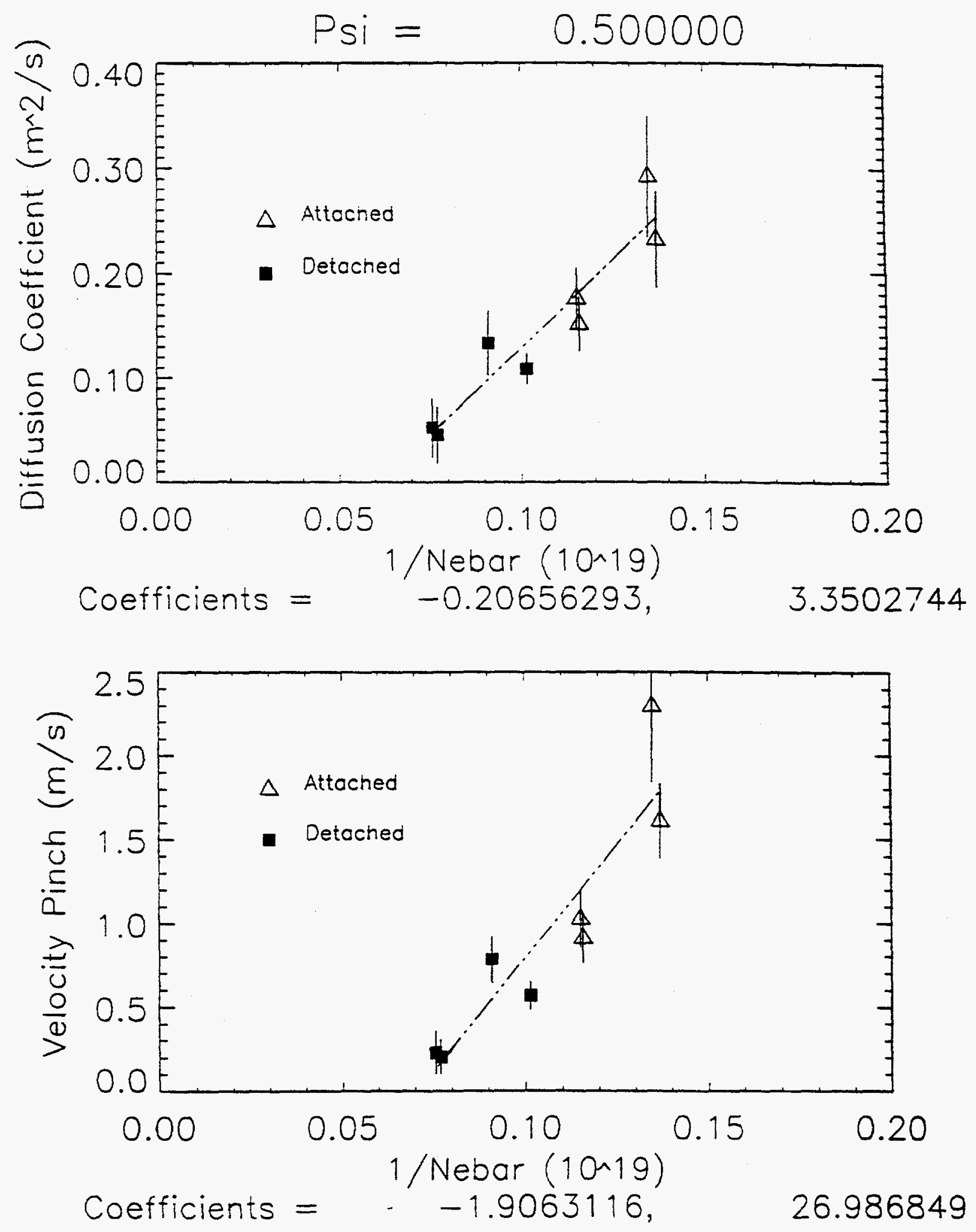

Figure1.4: Scaling of transport coefficients at $\Psi=0.5$ with the inverse density. 
central density was due to a change in the electron source term. Measurements of the $H_{\alpha}$ radiation showed an increase in the number of ionizations inside the last closed flux surface, LCFS, occurring after detachment. Numerical simulations estimated that an increase in the source by a factor of 2 would be consistent with the observed density profiles. The observed lack of change in the energy and impurity transport is also consistent with no change in the underlying transport. Thus, the experimental measurements indicate that the divertor detachment increases the electron source term inside the LCFS which results in the rise in central electron density.

\subsection{Thesis Outline}

This thesis can be divided into three distinct sections:

1) The design and construction of the two color interferometer, TCI.

2) The development and implementation of an inversion technique that provides useful density profiles and other processed data.

3) The performance of gas modulation experiments and the development of a transport analysis technique to determine transport coefficients, $\mathrm{D}$ and $\mathrm{V}$.

Each stage of the thesis will be described in detail. The introduction has presented the thesis topic and reviewed the phenomenology of previously observed enhanced confinement modes. Chapter 2 describes the Alcator C-Mod experiment and the relevant diagnostic systems that will be used. A historical perspective of the transport results from Alcator $A$ and $C$ is presented as well as a review of the initial results from the 1993 campaign. The two color interferometer is the fundamental diagnostic for electron transport studies. This diagnostic was designed and built specifically for Alcator C-Mod. The specifications of the optical layout, electronics and specific features are described in chapter 3. 
The inversion of chordal data from the interferometer into density profiles is a essential step. Because the view of the interferometer is limited to the central plasma, the determination of the edge density is crucial for the accurate measurement of the transport coefficients. In chapter 4 , the singular value decomposition (SVD) technique used to invert the phase measurements is discussed. The analysis for the selection of the appropriate weight for smoothing the inverted profile and the determination of the edge density is presented. The sensitivity of the inversion to the various assumptions is investigated. Two techniques to determine the diffusion coefficients have been developed and implemented. A regression analysis can be applied to the time histories of the profiles. Also a numerical simulation of the transport can be used to calculate the time dependent phase measurements and these results are compared with the actual measurements. The details of these two analyses are outlined in chapter 5 .

Experimental results are discussed in chapter 6 . The sensitivity of the diffusion coefficient profiles to various assumptions and systematic sources of error is examined. The diffusion coefficients determined by the regression technique are compared to those from the simulation. Interpretation of these results is presented in chapter 7.

Comparisons of energy, impurity and particle transport are made in order to formulate a consistent explanation for the rise in central density after detachment. Chapter 8 summarizes the findings of this thesis. 


\section{Chapter 2}

\section{Alcator C-Mod}

\subsection{Alcator C-Mod: Specifications and Mechanical Features}

Alcator C-Mod represents the third tokamak in a series of experimental machines at MIT designed to investigate the high field, high density operating regime. Alcator C, the immediate predecessor operated with toroidal fields, $\mathrm{B}_{\mathrm{T}}$, of greater than 9 Tesla and electron densities of $1.0 \times 10^{21} \mathrm{~m}^{-3}$. It achieved a record Lawson parameter, $\mathrm{n} \tau$, of $8.0 \times$ $10^{-19} \mathrm{~s} / \mathrm{m}^{-3}[56,57]$. Alcator C-Mod has built upon that experience and additional developments from other tokamak programs. The design parameters of Alcator C-Mod are:

\begin{tabular}{|c|c|}
\hline Toroidal Field $\left(\mathrm{B}_{\mathrm{T}}\right)$ & 9 Tesla \\
\hline Electron Density $\left(\mathrm{n}_{\mathrm{e}}\right)$ & $1.0 \times 10^{21} \mathrm{~m}^{-3}$ \\
\hline Plasma Current $\left(\mathrm{I}_{\mathrm{n}}\right)$ & $3 \mathrm{MA}$ \\
\hline Minor Radius (a) & $.21 \mathrm{~m}$ \\
\hline Major Radius $\left(\mathrm{R}_{0}\right)$ & $.67 \mathrm{~m}$ \\
\hline Plasma Elongation $(\kappa)$ & 1.8 \\
\hline
\end{tabular}

Table 1: Major Specifications of Alcator C-Mod [58].

Alcator C-Mod presently features $4 \mathrm{MW}$ of ICRF heating power ( $8 \mathrm{MW}$ in the future) and the ability to operate in either a closed upper or open upper divertor mode. A metal first wall constructed of molybdenum tiles constitutes the plasma facing surface. The tokamak was designed to provide ample access for diagnostics. There are 10 large 
horizontal ports that give access to the plasma midplane as well as two pairs of tangential ports. Twenty vertical ports are evenly spaced toroidally to provide additional views into the plasma.

The magnets for Alcator C-Mod are made of copper which are cooled by liquid nitrogen. This cooling reduces the resistance of the copper by up to a factor of 7 and helps to reduce both the required loop voltage and the ohmic heating of the coils themselves. A unique engineering feature is the sliding finger joints that join the different legs of the toroidal field (TF) coils. These joints perform the dual task of carrying the high currents necessary for high $\mathrm{B}_{\mathrm{T}}$ and relieving the stress due to magnetic forces during an experimental shot. Because of this multisectional TF coil design, Alcator C-Mod is able to use a single piece vacuum vessel which provides increased mechanical rigidity and integrity for the vessel. However, this also results in significant forces due to the induced eddy currents in the vacuum vessel. The effect of these currents on the magnetic field structure must be balanced by the external field coils. Alcator C-Mod employs 5 pairs of external field or control coils that control both the quality of the plasma null at breakdown as well as the vertical and radial position of the plasma. All except one pair of these coils are nested inside the toroidal field coils and the outer cylinder allowing for a very compact design. This small size increases the structural strength of the assembly in order to withstand the very large mechanical and thermal stresses during a shot.

Gas is injected into Alcator C-Mod through piezo-electric gas valves mounted on the horizontal ports. These valves are remote controlled and can be opened and closed on a millisecond timescale [33]. Additional fueling is supplied by a capillary gas system in the divertor. It is possible to use either set of valves in a continuous mode to provide background fueling or in a pulsed mode for oscillating gas puff experiments. Core fueling is also possible through a twenty barrel pellet injector capable of firing either $\mathrm{H}_{2}$ or $\mathrm{D}_{2}$ pellets. Figures 2.1-2.3 shows cross sectional views of the vacuum vessel and the locations 


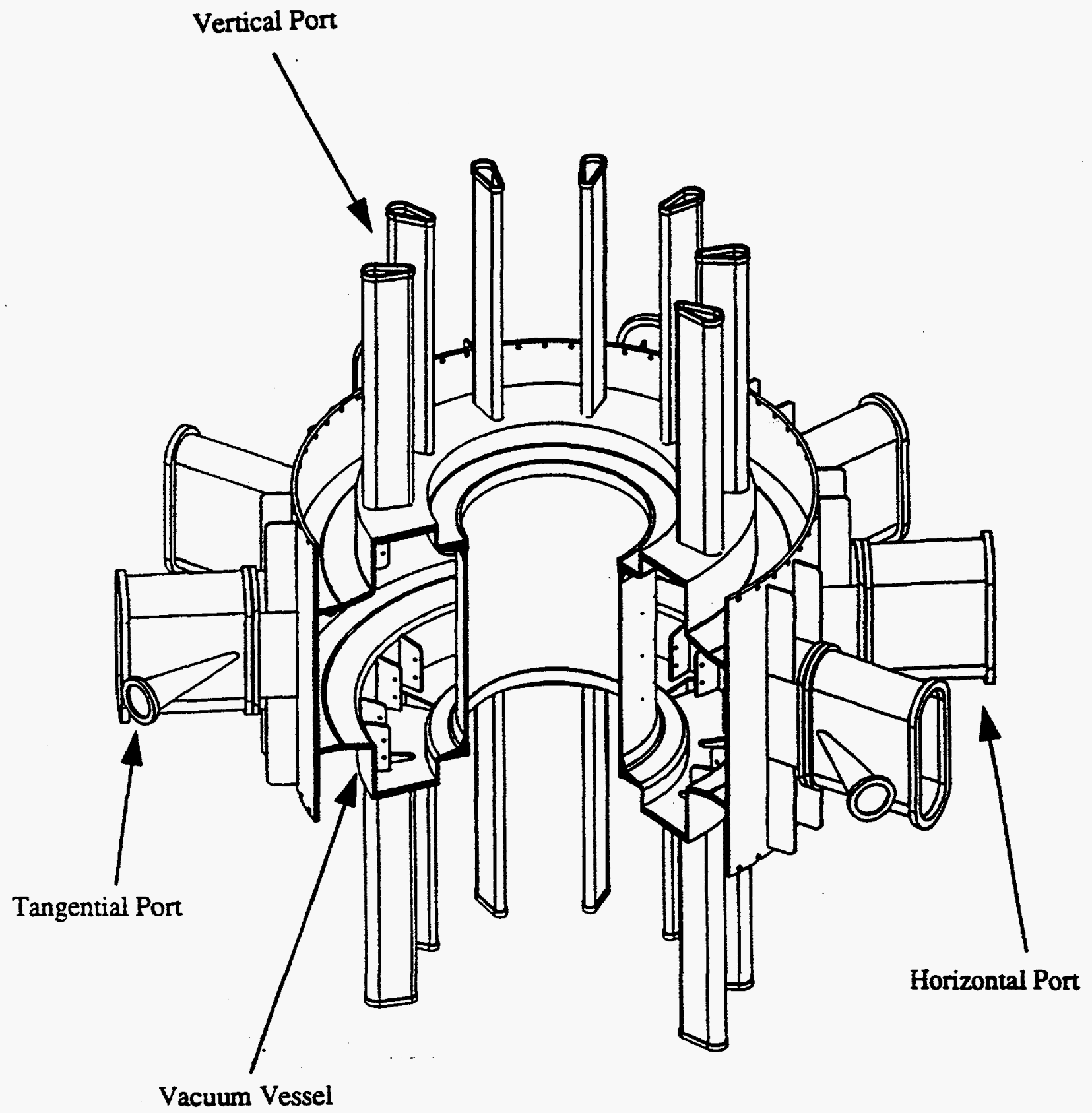

Figure 2.1: 3-D schematic of Alcator C-Mod with cross sectional view of the ports and vacuum vessel. 


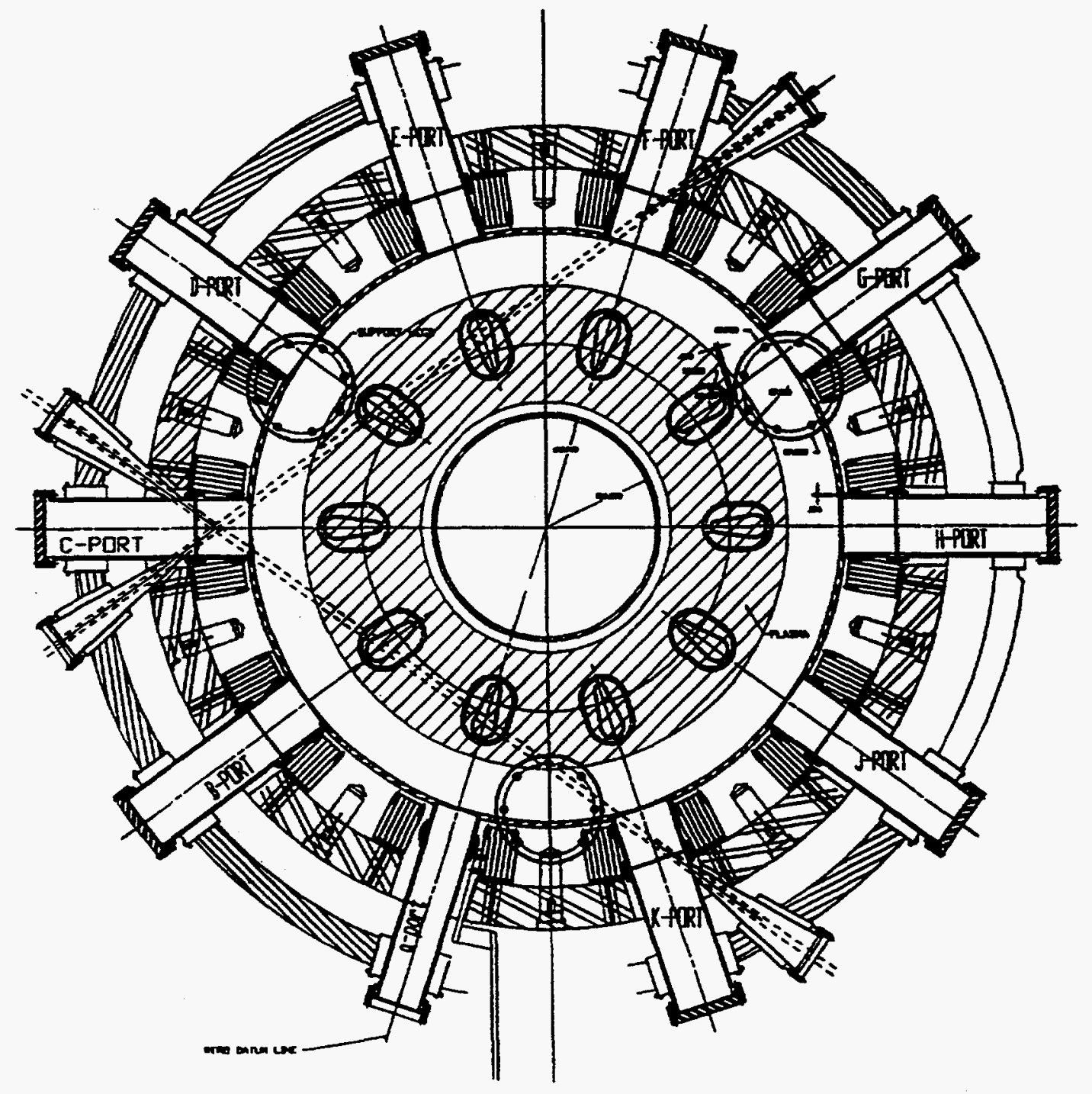

Figure 2.2: Cross Sectional view of Alcator C-Mod at the midplane. 


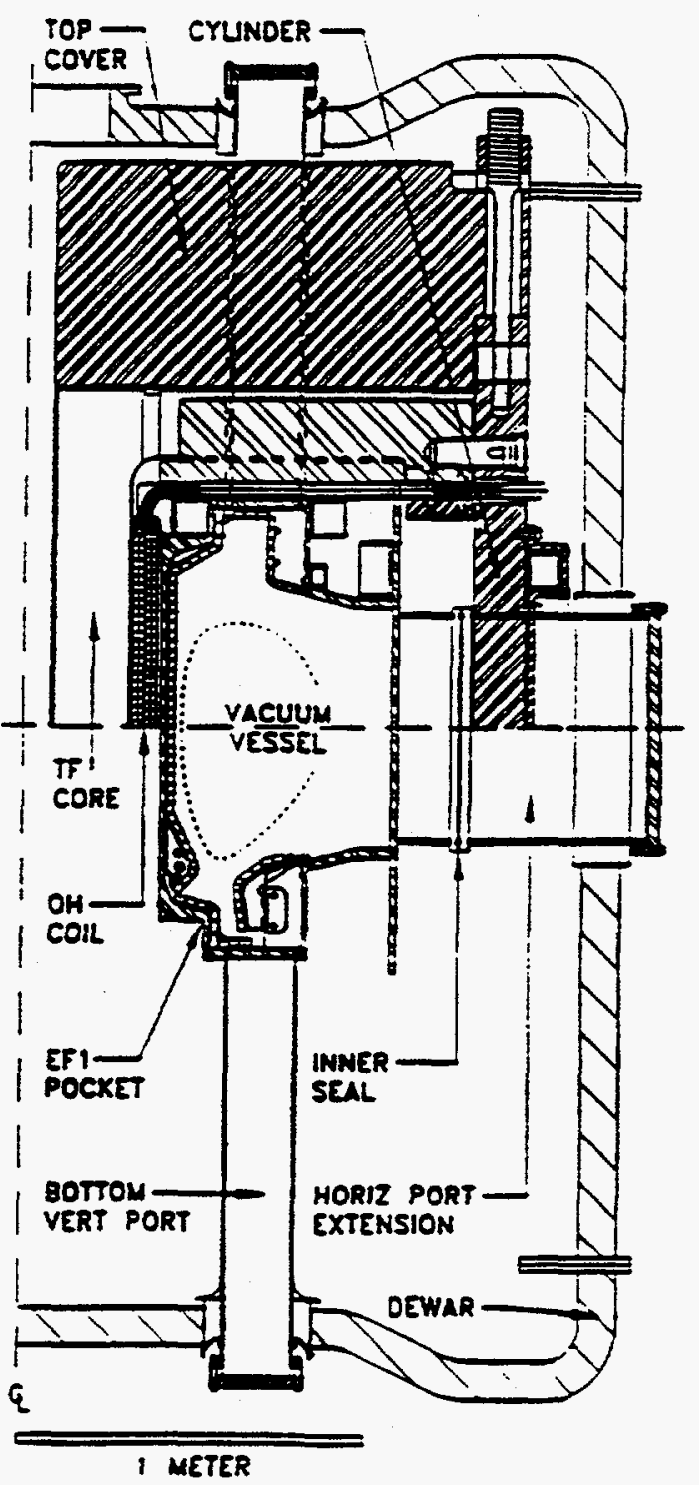

Figure 2.3: Vertical cross section of Alcator C-Mod showing the location of major system components. 


\section{Alcator C-Mod Crossection}

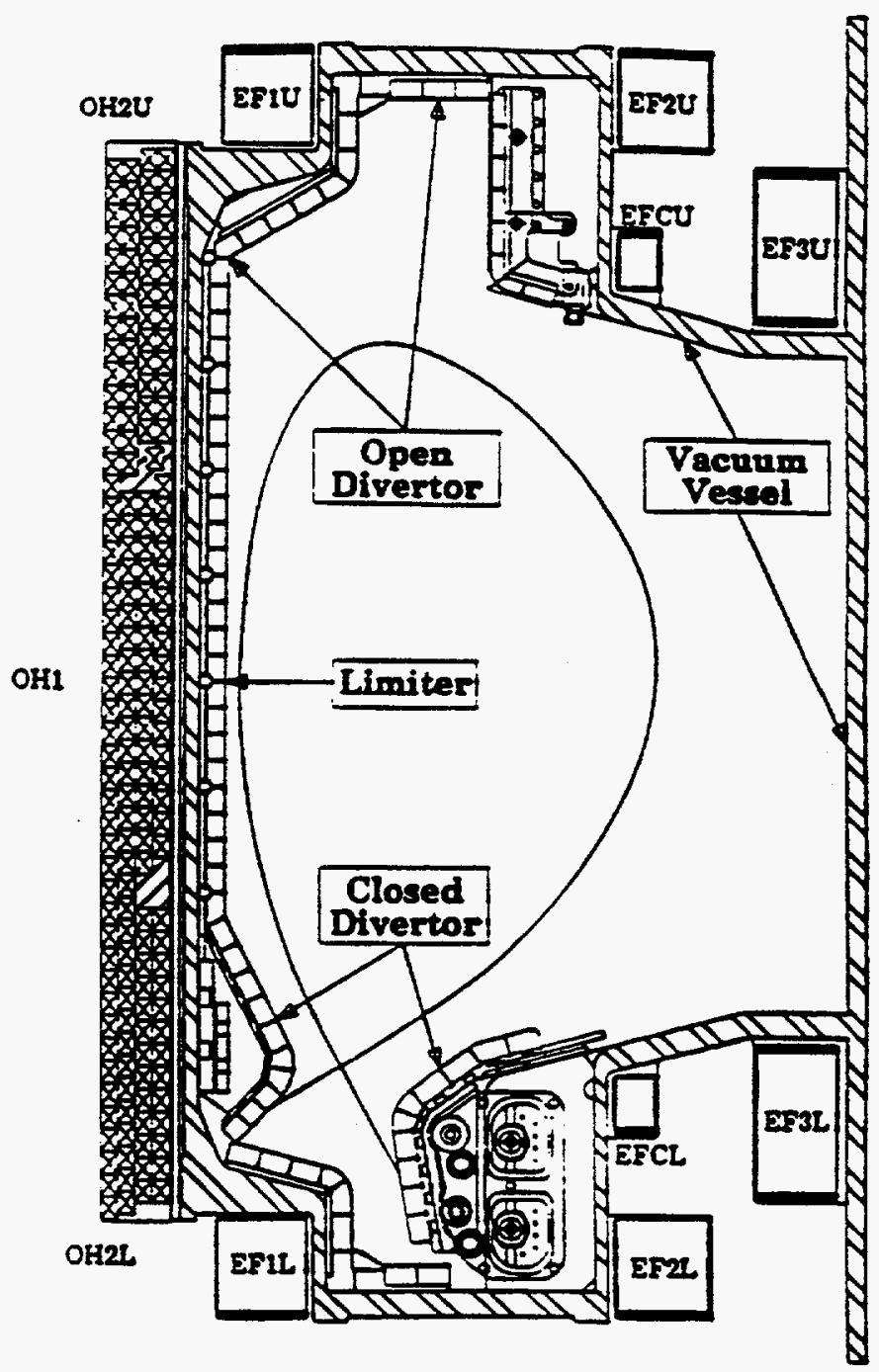

Figure 2.4: Cross sectional view of Alcator C-Mod showing the location of the external field coils and divertor [119]. Note the open configuration of the upper divertor. The lower divertor is in a closed configuration. 
of the diagnostic ports. Figure 2.4 presents the location of the divertor and external field coils relative to the vacuum vessel and plasma[58].

\subsection{Diagnostics on Alcator C-Mod}

To aid in the diagnosis of the plasma, an extensive set of dedicated diagnostics has been designed and built for Alcator C-Mod. Figure 2.5 gives a schematic of the location of each diagnostic relative to the machine. The diagnostics that were utilized in this thesis will be described in greater detail in this section.

\subsection{Microwave Reflectometer}

A four channel millimeter wave, amplitude modulated reflectometer was operational during the gas modulated runs. A reflectometer diagnostic is based on the principle that microwave radiation in the ordinary mode, $\mathrm{O}$-mode, has a critical density which reflects incoming beams. The value of the critical surface is given as a function of $\omega,[71]$

$$
n_{c}=\frac{\omega^{2} m \varepsilon_{0}}{e^{2}}
$$

Measurement of group phase delays provides information about fluctuations and density profiles. Receivers and transmitters are located on the midplane $15 \mathrm{~cm}$ from the LCFS. Spatial resolution of the measurements is $2 \mathrm{~cm}$ with temporal resolution of better than 10 ms. The system for Alcator C-Mod uses four frequencies at $50,60,75$ and $88 \mathrm{GHz}$ which correspond to electron densities of $3.13,4.6,6.5$ and $9.5 \times 10^{19} \mathrm{~m}^{-3}$. An amplitude modulation technique is applied to the signals to reduce the sensitivity to small fluctuations. Also, the use of an appropriate modulation frequency makes the system less sensitive to temporary loss of phase information [59]. A schematic of the reflectometer 


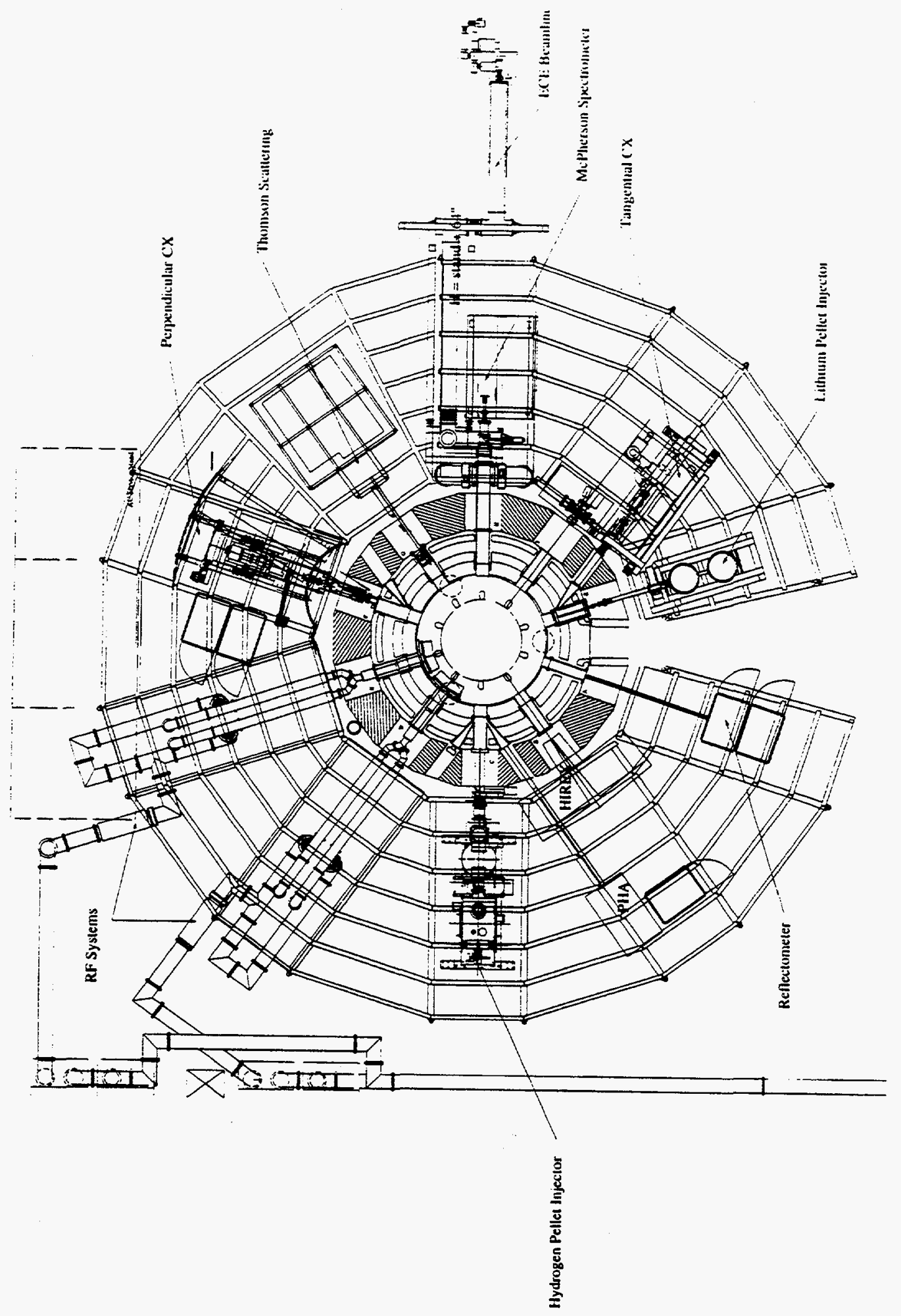

Figure 2.5: Location of the major diagnostics on Alcator C-Mod [117]. 


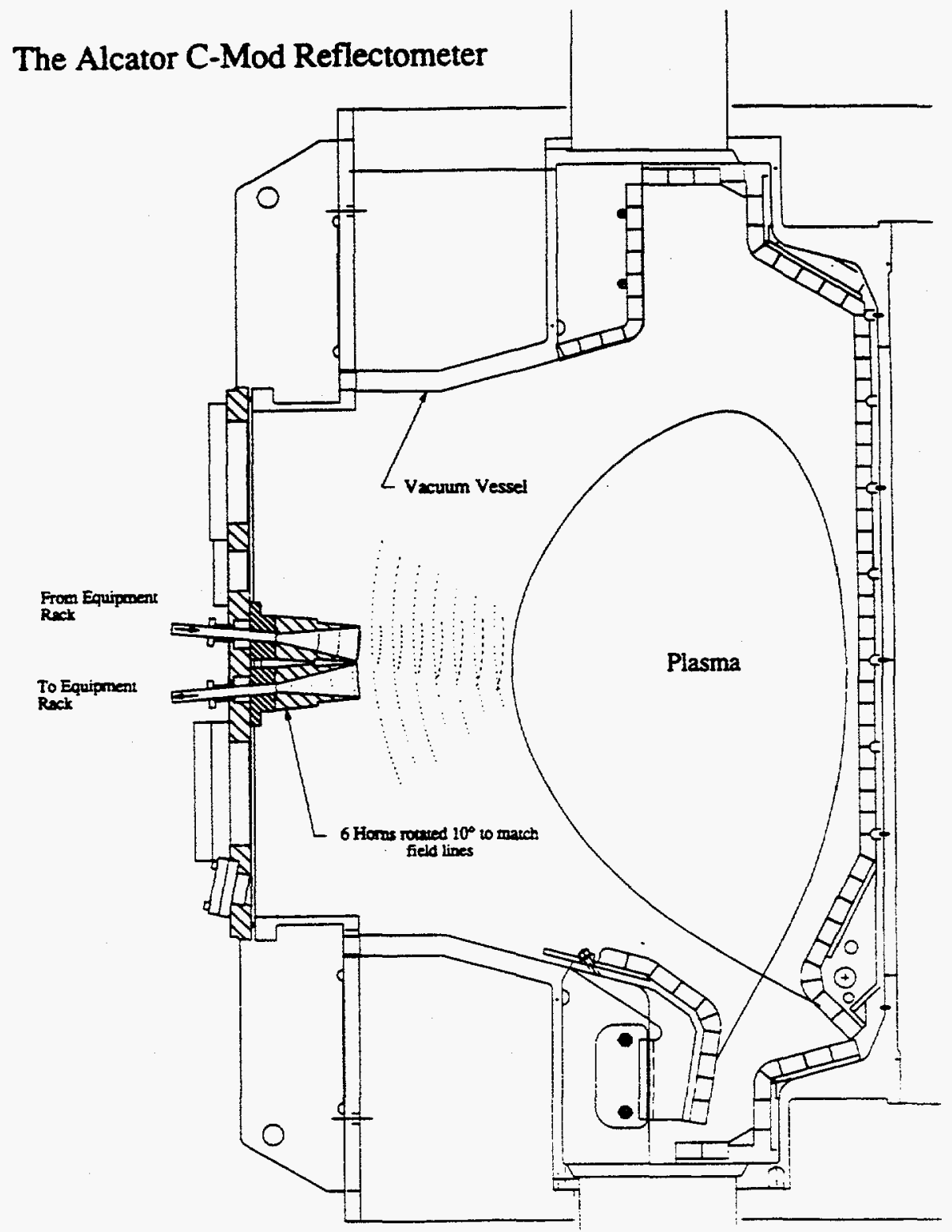

Figure 2.6: Schematic of Reflectometer system [59]. 
system is presented in fig. 2.6 .

\subsubsection{Limiter Probes}

Alcator C-Mod has two two-strap RF antenna capable of injecting up to $4 \mathrm{MW}$ of ICRF power. These antennas are located on $\mathrm{D}$ and $\mathrm{E}$ ports and are protected by two RF limiters. The limiters are located between ports $A$ and $B$ and $G$ and $H$. The limiter between $A$ and $B$ is a full limiter while the other is split with an open gap at the midplane. Embedded in each limiter are 8 Langmuir probes which are located at different toroidal and poloidal positions. Figure 2.7 shows a frontal view of the limiters and the probe locations in addition to a cross-sectional view of the limiters relative to the plasma. On the upper half of the full limiter, the lower set of probes is located $24.67 \mathrm{~mm}$ above the machine midplane. Figure 2.8 presents a schematic of the Langmuir probe embedded in the limiter tiles. These probes are operated in a swept configurations and can provide $\mathrm{n}_{\mathrm{e}}$ and $T_{e}$ measurements in the scrapeoff layer with a $20 \mathrm{~ms}$ time resolution.

\subsubsection{Fast Scanning or Reciprocating Probe}

A reciprocating probe has been installed of Alcator C-Mod for the purpose of measuring the spatial density and temperature profiles in the scrape-off layer.(see figure 2.9 ) This probe consists of four Langmuir probes attached to a pneumatically driven shaft that is fitted with stiff return springs so as to minimize the total round-trip time. ( $\approx$ $150 \mathrm{~ms}$ ) The entire moving assembly weights $2 \mathrm{~kg}$ and can reach a speed of $2.0 \mathrm{~m} / \mathrm{s}$. With a $7.5 \mathrm{~cm}$ travel, the fast scanning probe can provide measurements up to the LCFS. Since the probe will be subject to a hot plasma, it was designed to withstand a parallel heat flux of $50 \mathrm{~kW} / \mathrm{cm}^{2}$ which represents a safety margin of 2.5 . Within the probe tip assembly, four probes are available which can be operated in a variety of configurations. This is illustrated in fig. 2.10. In a triplet mode ( two probes biased and the third floating), continuous measurements of temperature, density and plasma potential are possible [55]. 


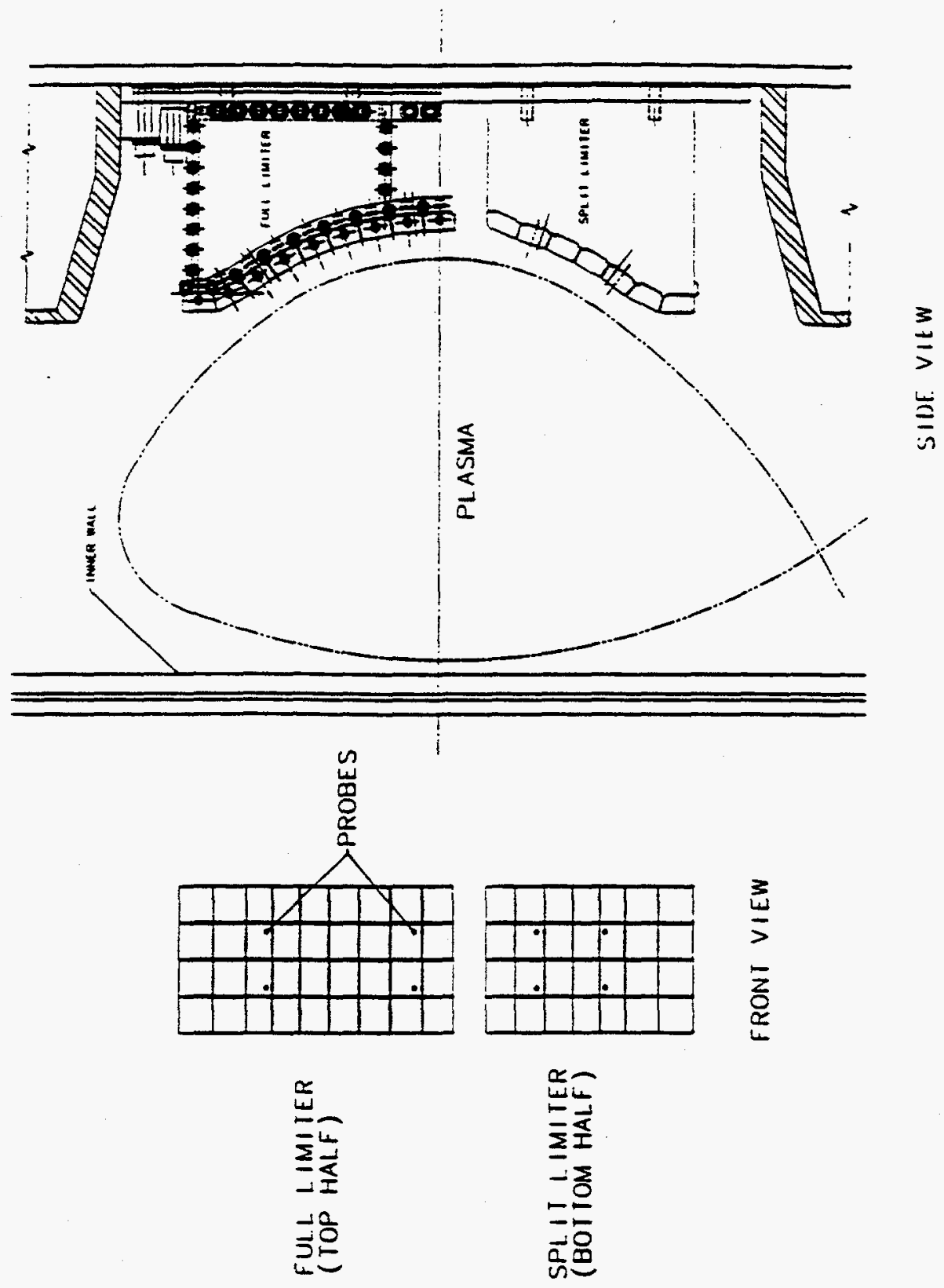

Figure 2.7: Front and side views of the RF limiters [118]. The upper half of the diagram represents a full limiter while the lower half shows a split limiter. 


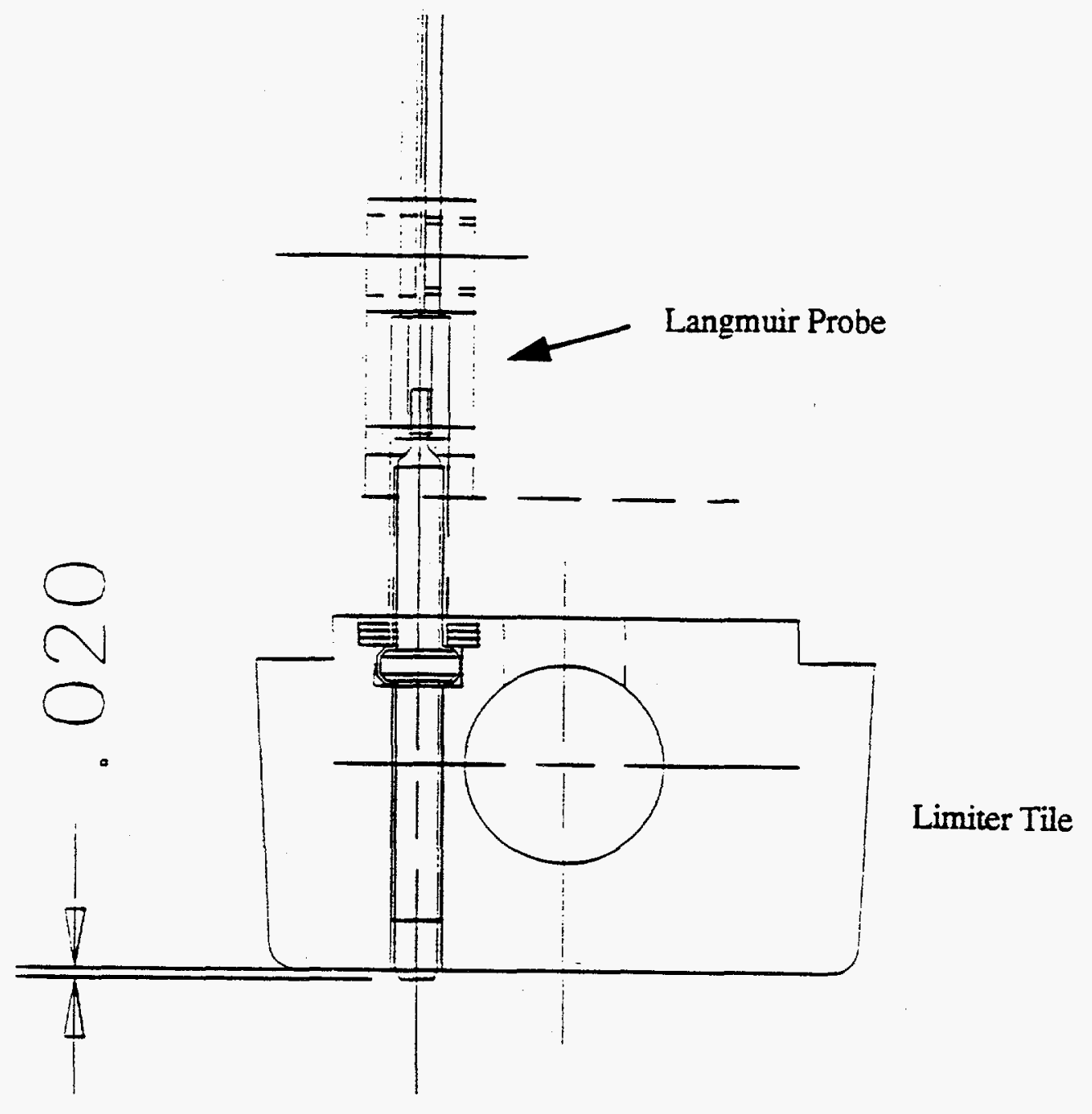

Figure 2.8: Schematic of the Limiter Probes [118]. 


\subsubsection{Divertor Probes}

The study of diverted plasmas and energy and particles flows into the divertor is a main objective of the Alcator C-Mod Program. As a result, a significant effort to diagnose the divertor plasma has been made. One of the main divertor diagnostics is the array of Langmuir probes recessed into the molybdenum tiles. Figure 2.9 indicates the location of these probes relative to the Alcator vacuum vessel and the fast scanning probe. There are sixteen sets of probes covering different poloidal locations in the divertor: six set are located on the inner divertor tiles and ten sets are on the outer divertor [60]. Figure 2.11 illustrates the probe design. The probes are aligned toroidally with each probe spaced 6.6 $\mathrm{mm}$ from the next. Probes are either flush with the tile surface or have a small dome projection for a greater collection area. Operation of these probes is similar to those in the fast scanning probe. They can be used in either a singlet, doublet or triplet mode in order to provide $n_{e}$ and $T_{e}$ at the divertor surface.

\subsubsection{Laser Ablation}

A laser ablation diagnostic has been installed on $\mathrm{J}$ port for impurity studies. This diagnostic consists of a $3 \mathrm{~J}$ Q-switched ruby laser that is incident on thin films $(\approx 1 \mu \mathrm{m})$ of metallic and non-metallic materials located $1 \mathrm{~m}$ from the plasma. The ablated material is blown into the plasma and by controlling the beam size or the beam energy, this provides a controlled source of impurities. For a typical spot size of $5 \mathrm{~mm}$ diameter, roughly $2 \times$ $10^{17}$ neutral atoms are ablated into the plasma [61].

\subsubsection{Spectroscopic Diagnostics}

For the observation of impurity line emission, a $2.2 \mathrm{~m}$ grazing incidence VUV spectrograph has been utilized. This diagnostic uses a micro-channel plate image intensifier and a 1024 element Reticon detector to view $50 \mathrm{~A}$ bands in the spectral range of 50-1000 A with a resolving power or 400-2000. Time resolution of the diagnostic is 


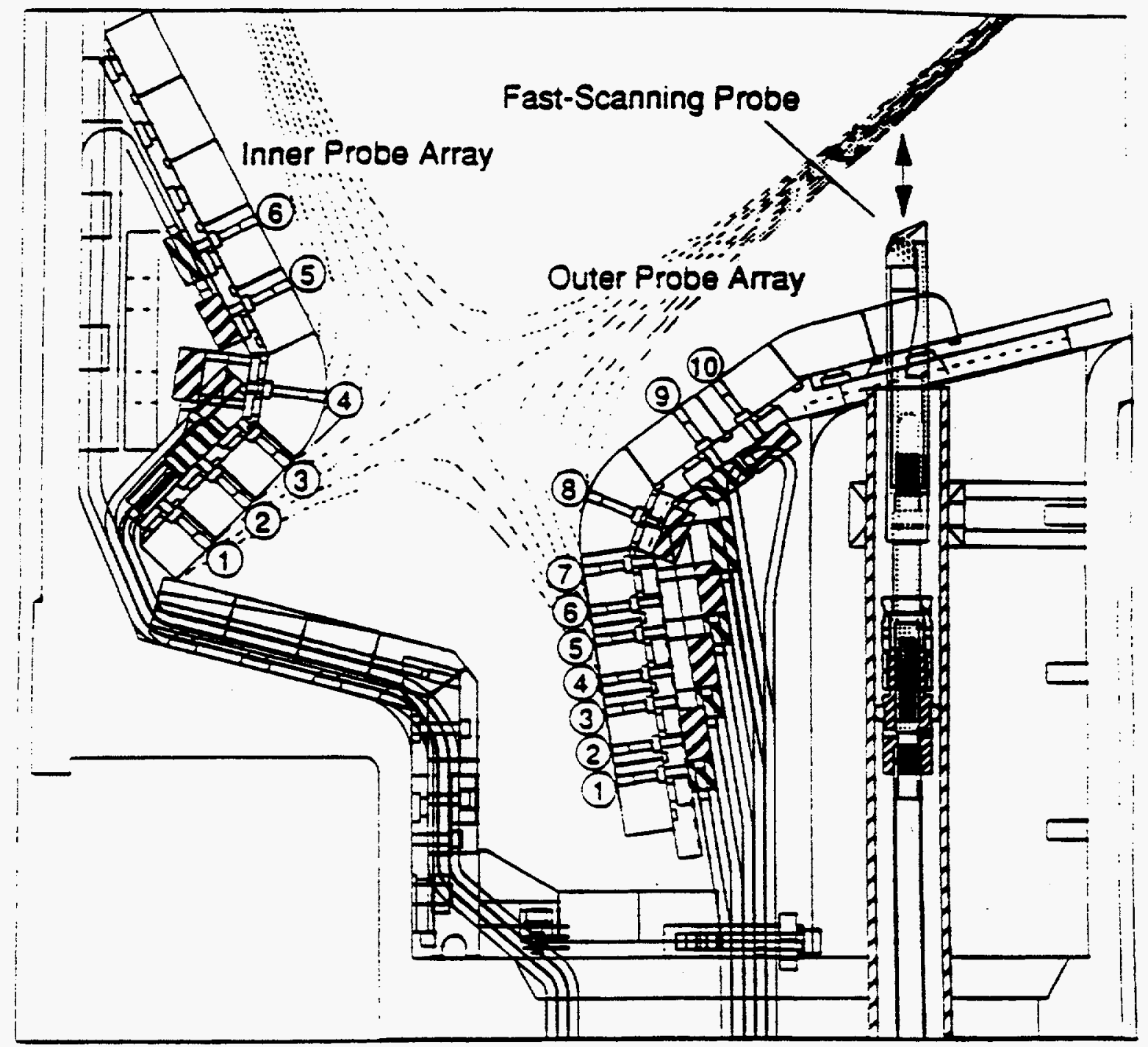

Figure 2.9: Location of reciprocating probe and divertor probes in the divertor [119]. 


\section{Fast-Scanning Probe Head Geometry}

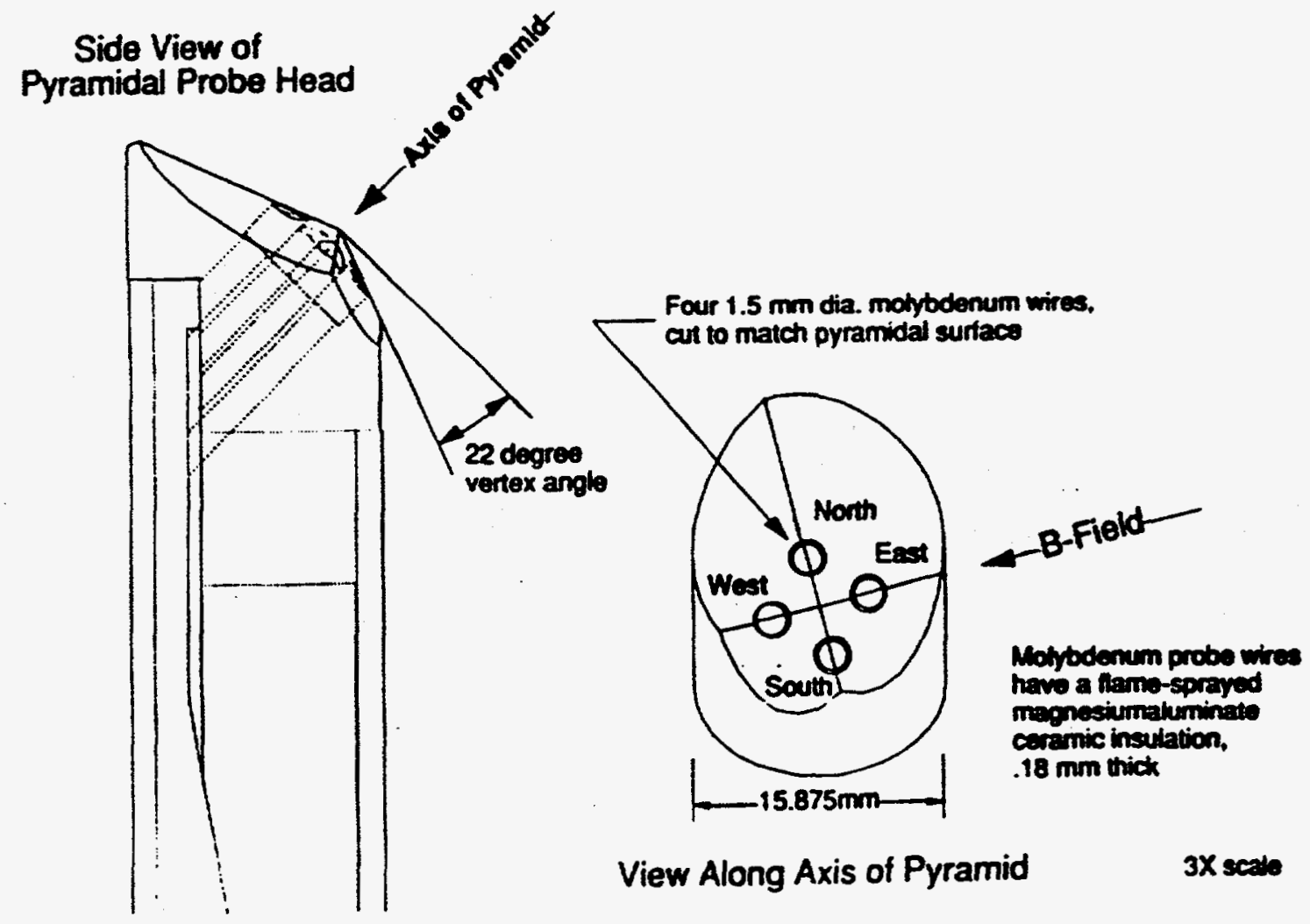

Figure 2.10: The four probe head of the reciprocating probe [119]. 


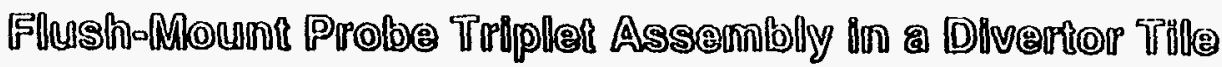

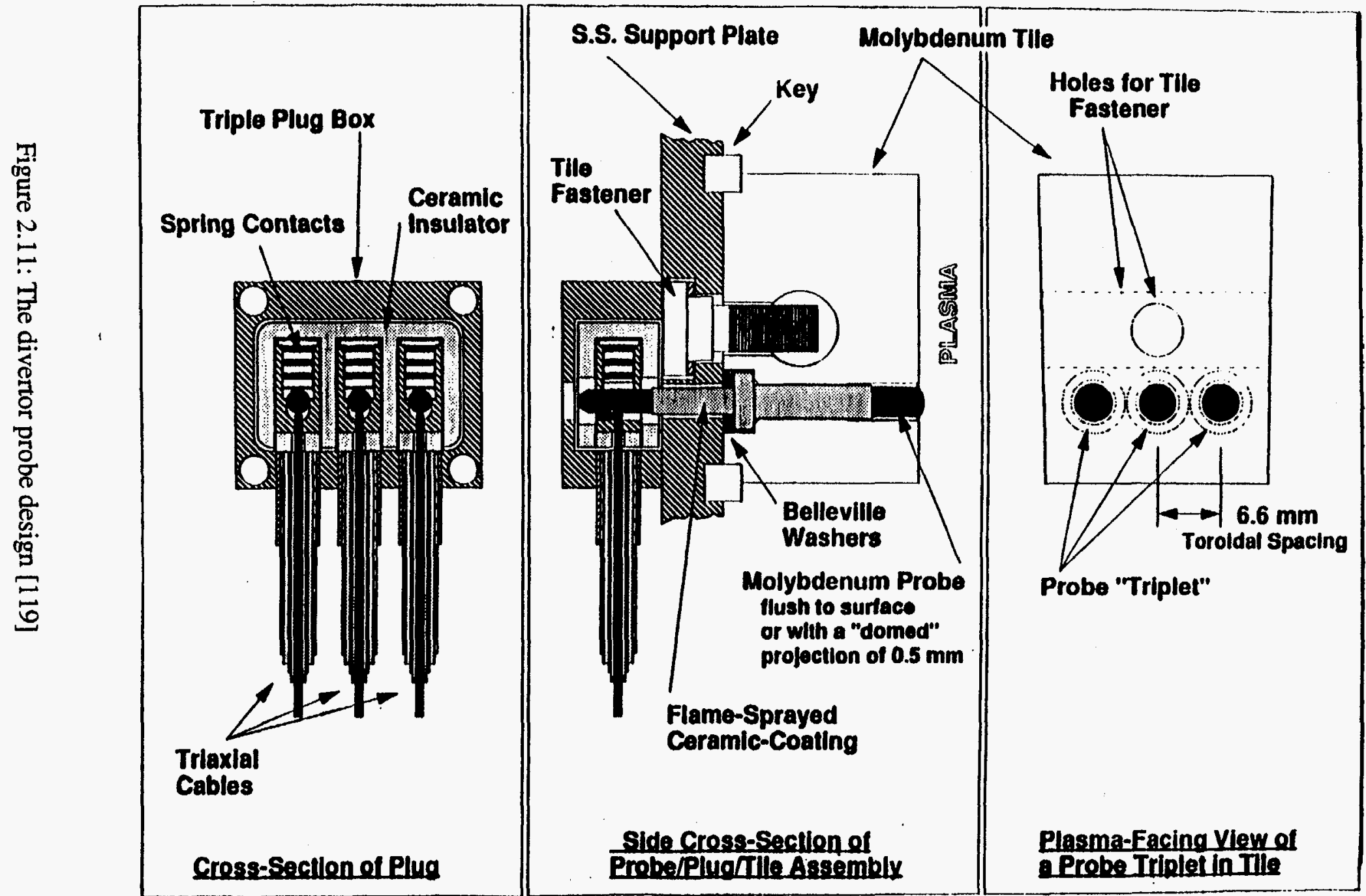


up to $0.5 \mathrm{~ms}$. The instrument is scannable and can view from $10 \mathrm{~cm}$ above to $25 \mathrm{~cm}$ below the midplane. The view range is illustrated in fig 2.12. The spectrograph has been absolutely calibrated with an accuracy of $15 \%$ up to $100 \mathrm{~A}$ and $30 \%$ up to $400 \mathrm{~A}$. This diagnostic can be used in concert with the five chord crystal x-ray spectrometer array measuring in the 2.5-4.0 A and the set of soft x-ray diode arrays to study impurity transport in Alcator C-Mod [62].

\subsubsection{Bolometry}

To measure the radiation from the visible to the soft $\mathrm{x}$-rays spectrum, a toroidally viewing bolometer array has been installed on the midplane. The diagnostic has 24 chords that cover from the inner wall to the outside edge of the plasma. Figure 2.13 gives a schematic of chord locations and a blow-up of one of the detector arrays. The vertical and horizontal positions of the arrays can be adjusted to change the view of the plasma. Spatial resolution is $2 \mathrm{~cm}$ and the time resolution is $1 \mathrm{~ms}$. The bolometer design is based on a gold foil absorber/Wheatstone resistance bridge concept. This allows measurement of incident powers as low as $50 \mu \mathrm{W} / \mathrm{cm}^{2}$. Inversion of the line integrated power measurements will provide details of the total power and emissivity profiles of the plasma.

In addition to the arrays in the main vacuum chamber, three additional arrays of 4 detectors each are located in the divertor. These arrays will provide information about the spatial distribution and magnitude of the total radiation from the divertor. The view of these arrays includes the $\mathrm{x}$-point of the plasma as well as the inner and outer divertor strike points, the positions on the divertor tiles where the field lines end. This is shown in figure 2.14 which shows the location of the divertor bolometer arrays [120].

\subsection{Transport Initiative}

The program to study tokamak transport processes has been an ongoing concern at MIT. Previous studies on Alcator $\mathrm{A}$ and Alcator $\mathrm{C}$ have already led to numerous 


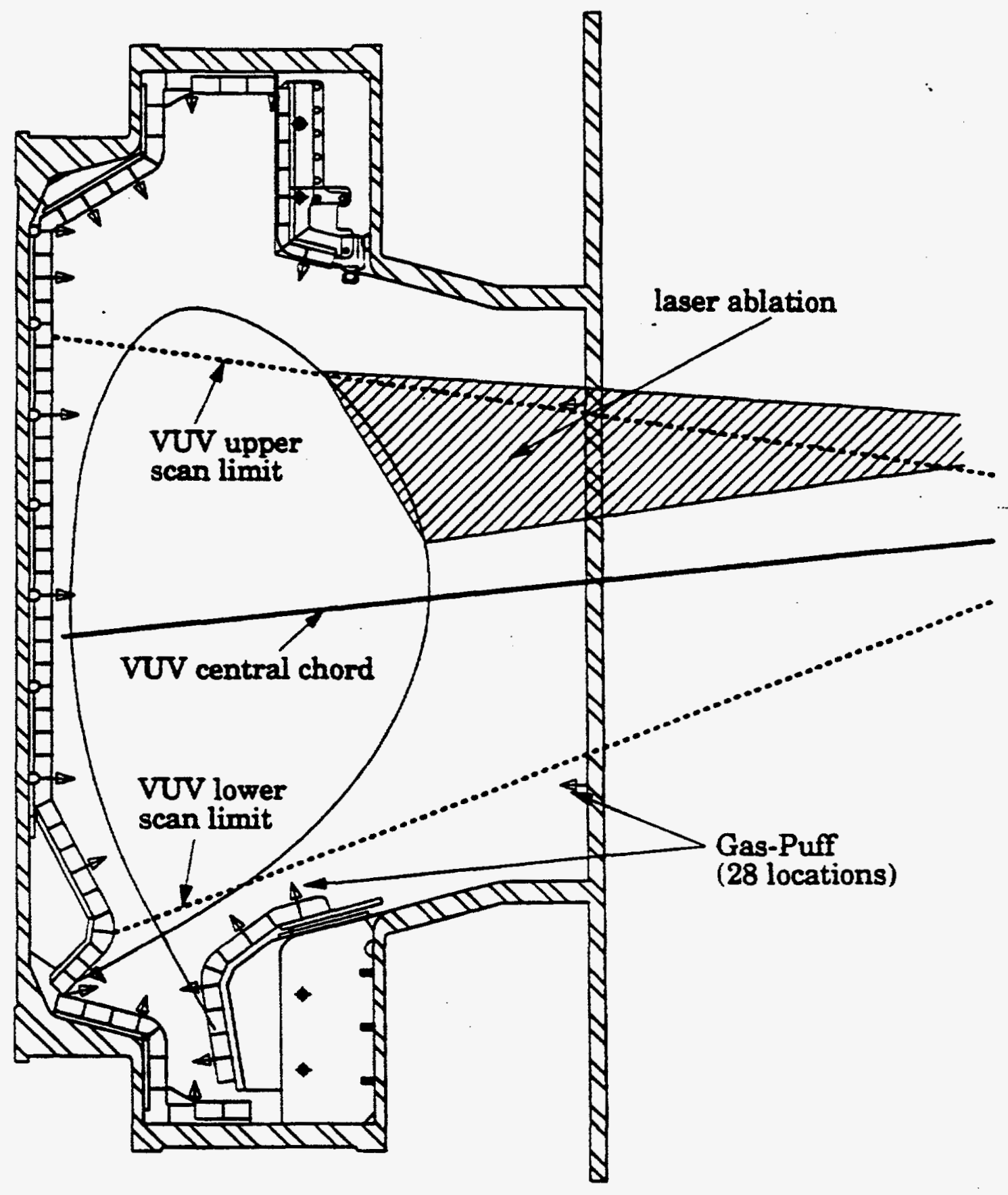

Figure 2.12: Layout of the view for the VUV Spectrometer [62]. 


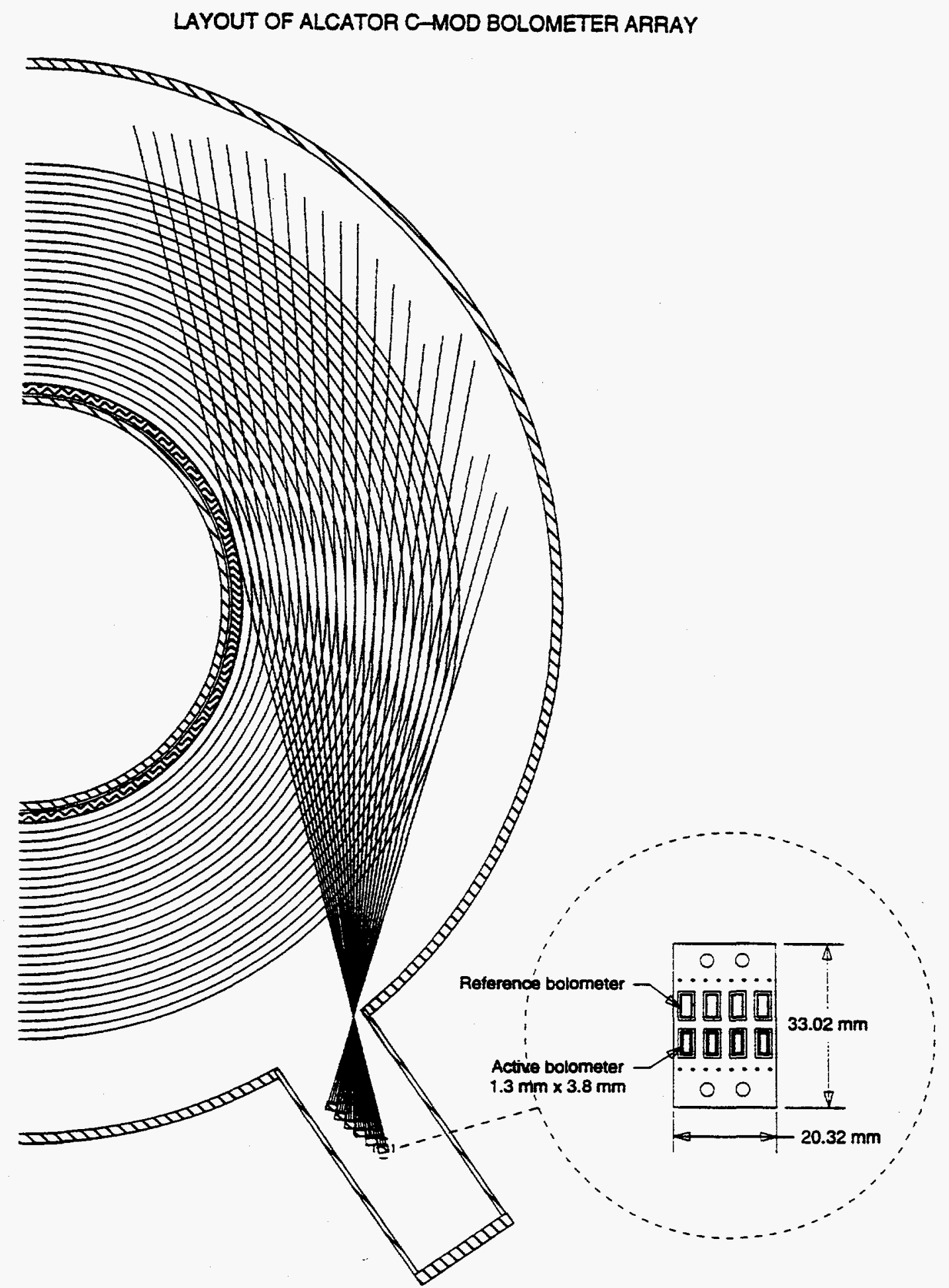

Figure 2.13: Location of the toroidally viewing bolometer array and corresponding chords at the midplane [120]. 


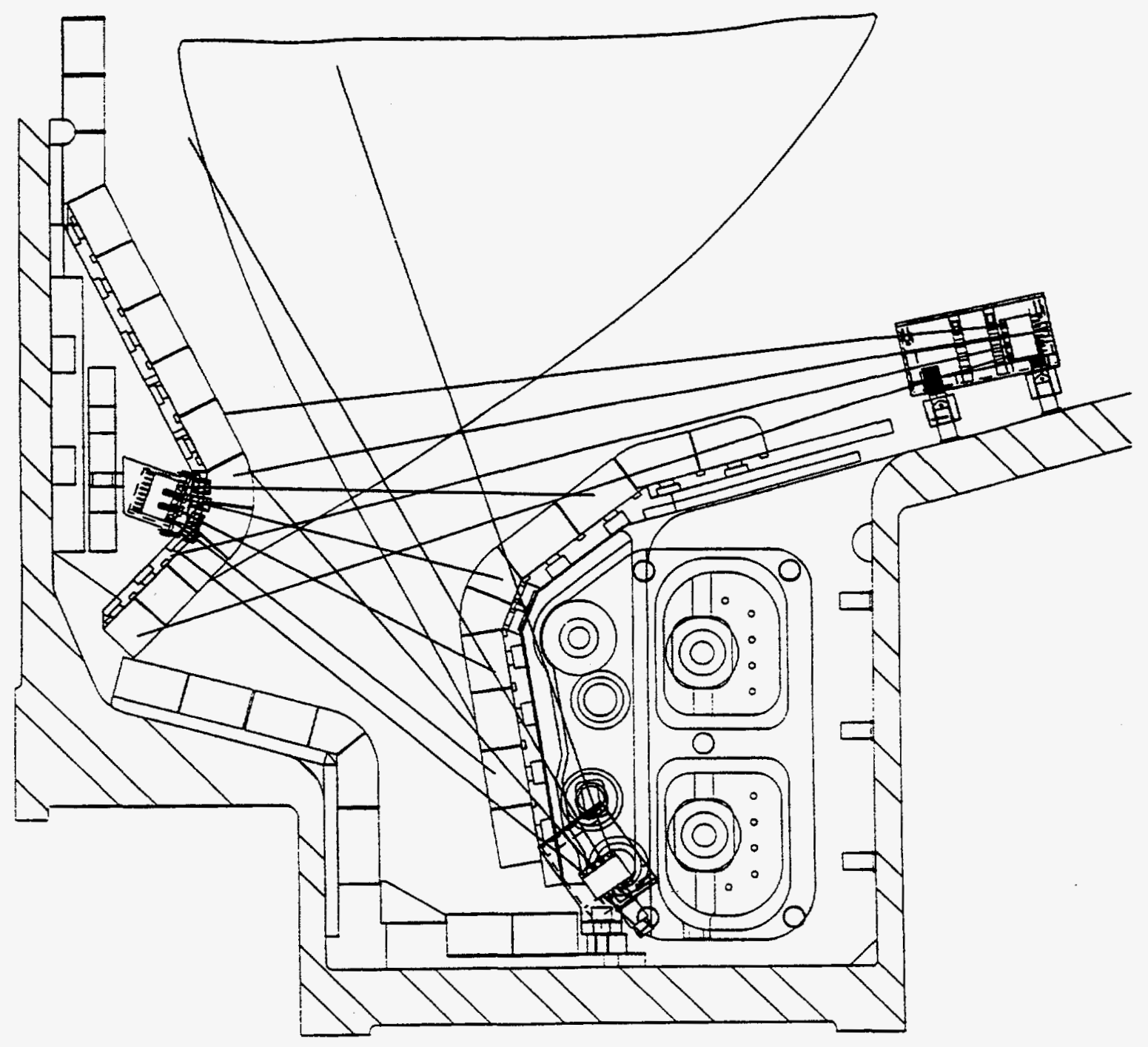

Figure 2.14: Location of the divertor bolometer arrays [120]. 
advances in the understanding of particle and energy transport. In this section, the achievements of these two machines at MIT will be reviewed in order to put Alcator CMod into some historical perspective.

\begin{tabular}{|c|c|c|}
\hline Plasma Parameter & Alcator A & Alcator C \\
\hline Minor radius (m) & 0.1 & $0.1-0.17$ \\
\hline Major Radius (m) & 0.54 & $0.58-0.71$ \\
\hline $\mathrm{B}_{\mathrm{T}}(\mathrm{T})$ & 9 & 13 \\
\hline $\mathrm{I}_{\mathrm{n}}(\mathrm{kA})$ & 300 & 800 \\
\hline $\mathrm{n}_{\mathrm{e} 0}\left(\mathrm{~m}^{-3}\right)$ & $1.0 \times 10^{21}$ & $2.0 \times 10^{21}$ \\
\hline $\mathrm{T}_{\mathrm{e} 0}(\mathrm{keV})$ & 2 & 3 \\
\hline
\end{tabular}

Table 2.2: Typical operating parameters of Alcator A and C [8].

\subsubsection{Alcator A}

Alcator A began operation in 1972 and was the first tokamak to achieve a Lawson parameter of $1 \times 10^{19} \mathrm{~s} \mathrm{~m}^{-3}[56,57,63]$. It was also the first tokamak to observe that the energy confinement scaled linearly with density. This behavior was contrary to the previous neoclassical prediction of an inverse density scaling. Instead, it was observed that electron energy diffusivity, $\chi_{e}$, scaled as $n_{e}{ }^{-1}$ and that $\chi_{e}$ ranges from 10 to 200 times the neoclassical value $[2,3,4]$. 


\subsubsection{Alcator C}

Continuation of experiments on Alcator $C$ resulted in the discovery of the saturation in $\tau_{\mathrm{e}}$ as $\bar{n}_{e}$ exceeds $2.5 \times 10^{20} \mathrm{~m}^{-3}$ [63]. At this density, although an increase in ion losses was predicted by neoclassical theory, an anomalously high loss rate was measured; an extra factor of 3 increase was necessary to explain experimental results [64, 65]. This anomaly scaled linearly with plasma current. Later experiments with the injection of hydrogen and deuterium pellets deposited $7.0 \times 10^{19} \mathrm{~m}^{-3}$ atoms directly in the core. The resulting peaked density profiles enabled the saturation of $\tau_{\mathrm{e}}$ as was seen with gas fueled shots to be overcome and the linear increase of $\tau_{\mathrm{e}}$ with $\bar{n}_{e}$ to be continued. In fact, for the higher densities, $\mathrm{n} \tau$ exceeded the $6.0 \times 10^{19} \mathrm{~s} \mathrm{~m}^{-3}$ required for break-even. This was the first example of the pellet enhanced confinement mode.

Another major result of Alcator $C$ was the investigation of the scaling of $\tau_{\mathrm{e}}$ with plasma parameters. A strong dependence with the major radius of the plasma was discovered and this in combination with findings from other experiments culminated in the Neo-Alcator scaling [66],

$$
\tau_{e}^{N e o}=1.92 \times 10^{22} \bar{n}_{e} R^{2} a^{1.04} .
$$

This result represented the first experimental support for a dependence of $\tau_{e}$ on the major radius.

The Alcator program has always been at the forefront of transport studies and Alcator C-Mod continues that long and successful commitment. While, the effort has previously been focused on energy confinement, the unique set of high spatial and temporal resolution diagnostics on Alcator C-Mod offers an ideal opportunity to continue and extend these studies to particle transport. 


\subsection{Initial Results}

Alcator C-Mod began operation as a research tokamak in May 1993. Since then, the initial campaign of 1993 has been completed and a summary of results will be presented. The campaign was quite successful with advances in all program objectives being made [54]. Typically, the machine operated at a magnetic field of $5.3 \mathrm{~T}$, a plasma current of up to $1 \mathrm{MA}$, plasma densities up to $9 \times 10^{20} \mathrm{~m}^{-3}$ and electron and ion temperatures of 2.6 and $1.6 \mathrm{keV}$ respectively. Various phenomena such as "snakes", a localized $\mathrm{m}=1$ density phenomena, and marfes, a phenomena characterized by poloidally asymmetric radiation from the edge, have been observed [112]. Operation in both limited and diverted mode has been achieved. An e-folding length of $1.5 \mathrm{~mm}$ in the scrapeoff layer close to the LCFS has been measured. This length increases to $4 \mathrm{~mm}$ further out radially. Divertor studies have observed the phenomena of both localized and fully detached plasmas on Alcator C-Mod. Finally, ICRF experiments with a single strap antenna were performed and up to $0.75 \mathrm{MW}$ of RF power have been coupled to the plasma [67].

In the realm of transport studies two interesting results have emerged. The first is the discovery that in ohmic operation, Alcator C-Mod behaves in a much different manner than Alcator-C. The previous experiment obeyed Neo-Alcator scaling which has a strong dependence on density. However, even though many of the engineering parameters (e.g. minor radius) are comparable, Alcator C-Mod confinement exhibits little dependence on density, rather, a strong dependence on the plasma current is observed. Figure 2.15 illustrates the better fit of $\tau_{E}$ with the ITER89-P or L-Mode scaling,

$$
\tau_{E}=0.048 I_{p}^{0.85} R^{1.2} a^{0.3} \kappa^{0.5} B^{0.2} n_{e}^{0.1}\left(\frac{m_{i}}{m_{p}}\right)^{0.5} P^{-0.5},
$$


$T_{E}$ VS $T_{\text {ITER89- }}$
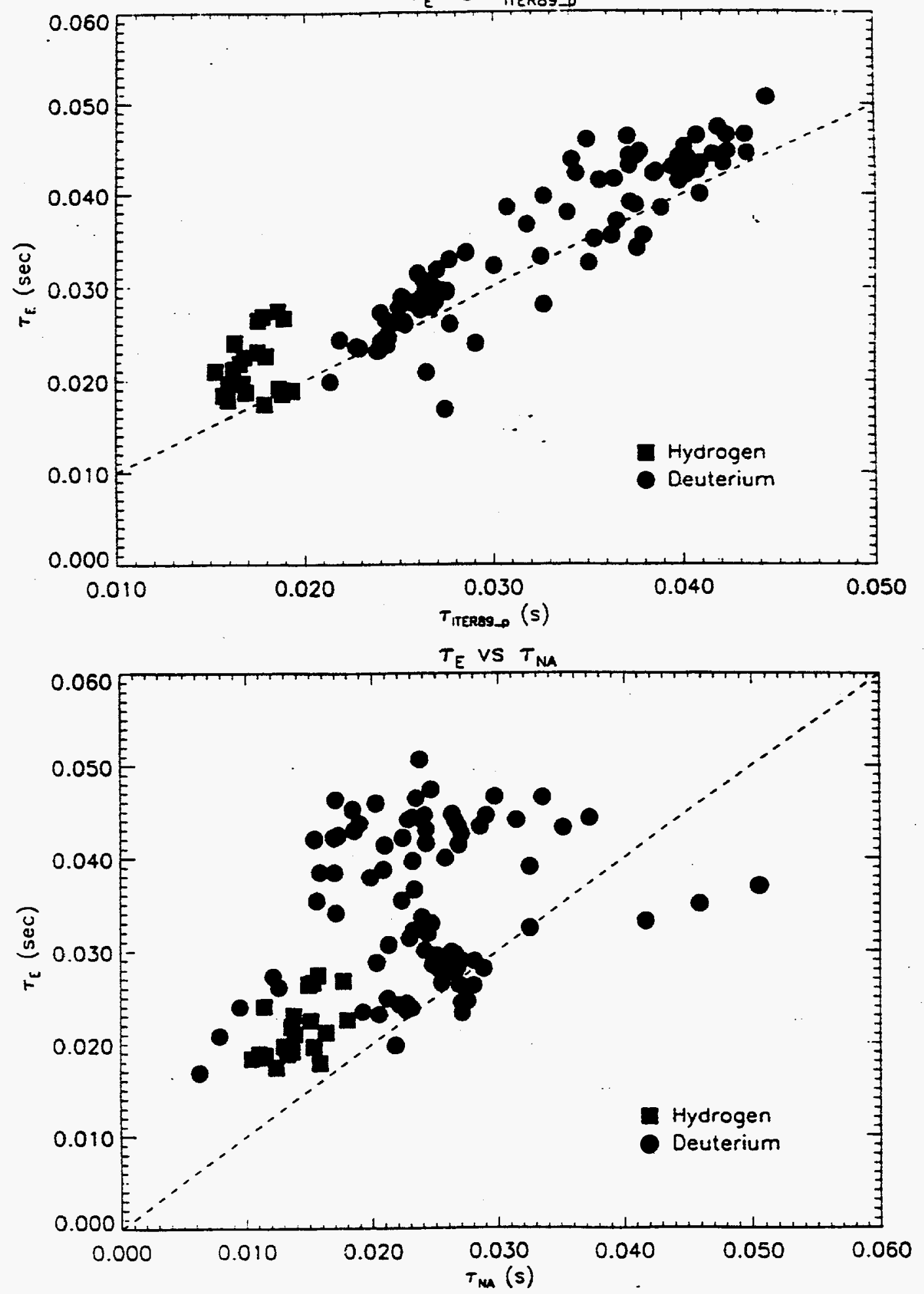

Figure 2.15: Comparison of the measured energy confinement time on Alcator C-Mod during the 1993/1994 campaign with L-Mode and Neo-Alcator scaling [68]. 
than with neo-Alcator scaling. In eqn. $1.2, \mathrm{I}_{\mathrm{p}}$ is the plasma current in $\mathrm{MA} ; \mathrm{R}$ is the major radius; $a$ is the minor radius; $K$ is plasma elongation; $B$ is the magnetic field; $m_{i}$ is the ion mass; $\mathrm{m}_{\mathrm{p}}$ is the proton mass; $\bar{n}_{e}$ is the line averaged electron density in units of $10^{20} \mathrm{~m}^{-3}$; and $P$ is the power input in $M W[68]$.

For lower density plasmas, confinement in Alcator C-Mod can exceed that of NeoAlcator by a factor of 2 . Reasons for this improvement are yet to be determined but several notable differences between Alcator-C and Alcator C-Mod are: 1) the shaping of the plasma, elongated vs. circular, 2) the limiter configuration, and 3) the proximity of the vacuum chamber.

The other notable observation during the first campaign is the achievement of ohmic H-Modes. Contrary to the prediction from other tokamaks that indicated that high field, high density machines would have difficulty achieving H-Modes [69], $\mathrm{H}$-modes in Alcator have been observed with $\mathrm{B}_{\mathrm{T}}$ up to $5.25 \mathrm{~T}, \bar{n}_{e}$ up to $1.4 \times 10^{20} \mathrm{~m}^{-3}$ and less than 1MW of total input power. ELM-free H-modes have been achieved with toroidal fields ranging from $2.9<\mathrm{B}_{\mathrm{T}}<3.2 \mathrm{~T}$ and allowable densities falling between $0.75<\bar{n}_{e}<1.1 \times$ $10^{20} \mathrm{~m}^{-3}$ while ELMy H-modes were seen at higher $\mathrm{B}_{\mathrm{T}}$ and density $\left(0.9<\bar{n}_{e}<1.4 \times\right.$ $10^{20} \mathrm{~m}^{-3}$ ) [69]. Marked by the characteristic drop in $\mathrm{H}_{\alpha}$ radiation, a rise in central density and steeper edge gradients, Alcator C-Mod $\mathrm{H}$-modes typically last $50 \mathrm{~ms}$ with increases in $\tau_{\mathrm{E}}$ of $60 \%$. The H-mode thresholds on Alcator C-Mod agree well with the power scaling from ASDEX-Upgrade and DIII-D(see figure 2.16) [68],

$$
P=4.4 \times 10^{-3} \bar{n}_{e} B_{T} S
$$

where $\mathrm{P}$ is the power input in MW; $\mathrm{B}$ is the magnetic field in Tesla; $\bar{n}_{e}$ is the line averaged electron density in unit of $10^{19} \mathrm{~m}^{-3}$; and $\mathrm{S}$ is the plasma surface area. H-Modes with Li pellet injection can exhibit a threshold that is lower by a factor of 2 . 


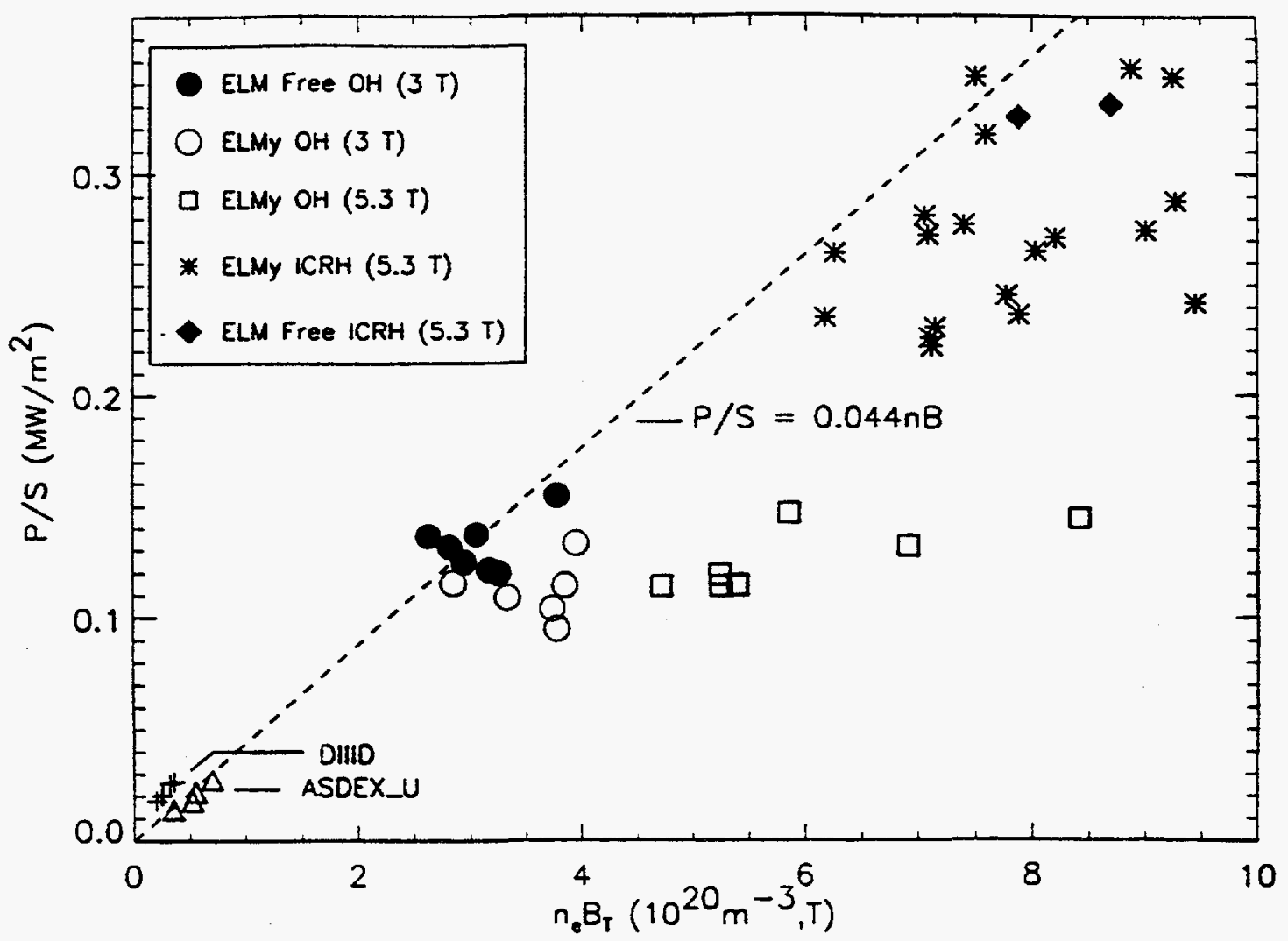

Figure 2.16: Comparison of H-Mode threshold on Alcator C-Mod with the power scaling from ASDEX-Upgrade and DIII-D [68]. 


\section{Chapter 3}

\section{Two Color Interferometer System}

\section{Introduction}

One of the baseline diagnostics on Alcator C-Mod is the two-color interferometer or TCI. This instrument was designed and built specifically for Alcator in order to provide density information with a fine spatial and temporal resolution. In addition to providing profile information, the TCI supplies a signal to the hybrid control system for density feedback. Ten $\mathrm{CO}_{2}$ and four $\mathrm{HeNe}$ channels view the central $20 \mathrm{~cm}$ of the plasma through a vertical port. Figure 3.1 shows the location of the beam relative to the plasma and vacuum vessel. The interferometer is in a two pass Michelson configuration. ECL electronics used in the phase demodulators allow a time resolution of up to $1 \mu \mathrm{s}$ which is fast enough to follow pellet injection without loss of signal. The TCI can follow fringes with a theoretical resolution of $0.08 \mathrm{CO}_{2}$ degrees which corresponds to $2.3 \times 10^{16} \mathrm{~m}^{-2}$ in integrated line density. This chapter will review the design and special features of the TCI system.

\subsection{Issues in Interferometer Design}

In the design of an interferometer, there are several issues that need to be considered. References by Veron [70] and Hutchinson [71] summarize these concerns. The three main factors that must be balanced in interferometer design and wavelength selection are:

1) Signal Size,

2) Refraction,

3) Vibrational Error. 


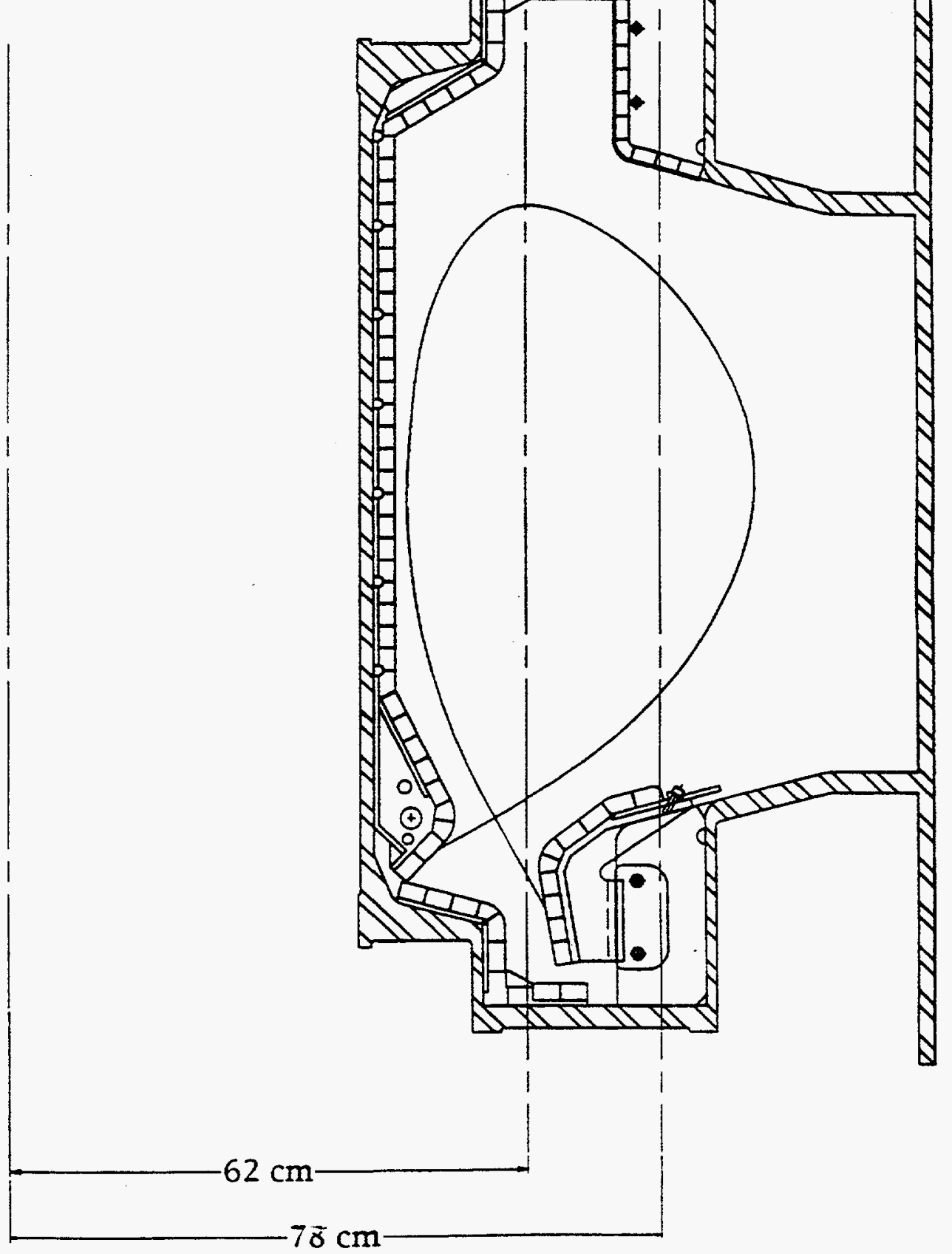

Figure 3.1: Location of TCI chords relative to the plasma. 


\subsubsection{Signal Size}

In general, for the frequencies used as probing beams, the ordinary -mode ( $\mathrm{O}$ mode) dispersion relationship is described by [71],

$$
n^{2}=\frac{c^{2} k^{2}}{\omega^{2}}=1-\frac{\omega_{p}^{2}}{\omega^{2}}
$$

Using this definition for the index of refraction, the phase shift induced by the plasma is given by

$$
\Delta \phi=\int \Delta k d l=\frac{\omega}{c} \int \Delta n d l .
$$

For the interferometer, the phase shift is due to the effect of the plasma so

$$
\Delta n=n_{\text {plasma }}-n_{\text {air }}
$$

For a typical probing frequency $\omega^{2}>\omega_{p e}^{2}$ so eqn 3.1 can be expanded as

$$
n=1-\frac{1}{2} \frac{\omega_{p}^{2}}{\omega^{2}}
$$

Thus, the phase shift can be written as

$$
\begin{aligned}
& =\frac{\omega}{c} \int\left(1-\frac{1}{2} \frac{\omega_{p}^{2}}{\omega^{2}}-1\right) d l \\
& =\frac{\omega}{c} \int \frac{\omega_{p}^{2}}{2 \omega^{2}} d l
\end{aligned}
$$




$$
=\frac{\lambda e^{2}}{4 \pi m \varepsilon_{0} c^{2}} \int n_{e} d l
$$

Thus, the measurable phase shift is proportional to the free space wavelength, $\lambda$, which should then be chosen as large as possible to guarantee a sufficient signal to noise.

\subsubsection{Vibrational Error}

In any optical system there will be many optical elements that can contribute to the error in the signal. Any movement of a lens or mirror that changes the effective pathlength of the beam will appear as a phase shift of magnitude

$$
\Delta \phi=\frac{\Delta l}{\lambda} .
$$

Again, a large wavelength will reduce the error due to these vibrations.

\subsubsection{Refraction}

The final consideration concerns refractive effects when a beam passes through a plasma. Because access to the plasma is usually through a port which is limited, when the beam is subject to very large density gradients it may be refracted. This loss of signal level can affect measurement of such phenomena like pellet injection. The amount of refraction is proportional to $\lambda^{2}$ and is given by [72],

$$
\theta(\rho)=-\frac{\rho \lambda^{2} e^{2}}{4 \pi^{2} c^{2} \varepsilon_{0} m} \int_{0}^{\sqrt{a^{2}-\rho^{2}}} \frac{1}{r} \frac{d n}{d r} d z,
$$

where $\rho$ is the minor radius at which the chord intercepts the midplane. 

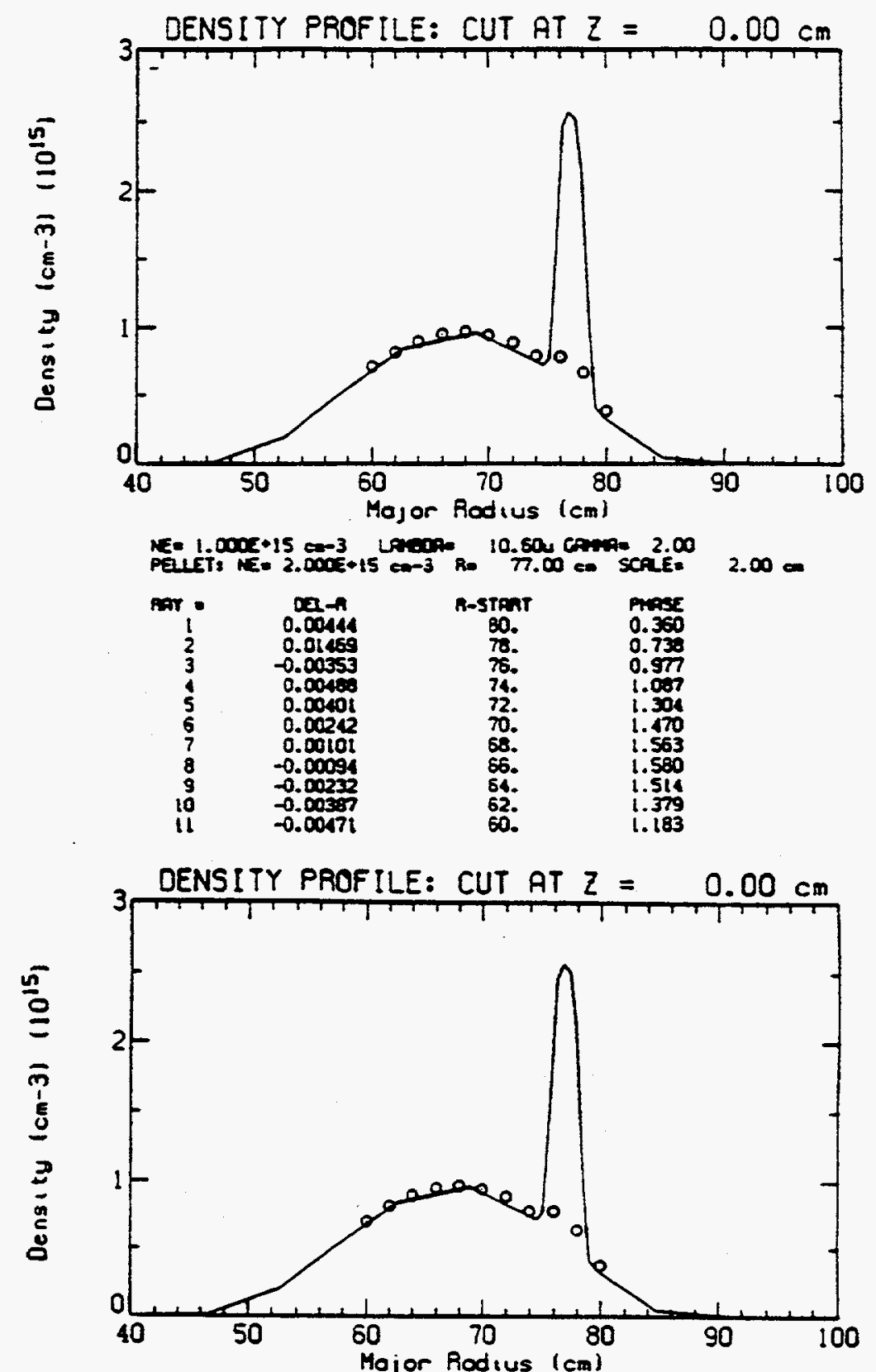

\begin{tabular}{|c|c|c|c|}
\hline $\begin{array}{c}\text { Par } \\
1 \\
2 \\
3 \\
4 \\
5 \\
6 \\
7 \\
8 \\
9 \\
10 \\
11\end{array}$ & $\begin{array}{r}04-\mathrm{A} \\
0.56950 \\
1.84057 \\
-0.38160 \\
0.03928 \\
0.50465 \\
0.31295 \\
0.12753 \\
-0.11846 \\
-0.29004 \\
-0.48757 \\
-0.80455\end{array}$ & $\begin{array}{c}\text { A-st6. } \\
80 . \\
78 . \\
76 . \\
74 . \\
72 . \\
70 . \\
68 . \\
65 . \\
64 . \\
62 . \\
60 .\end{array}$ & $\begin{array}{l}\text { Prase } \\
3.951 \\
7.644 \\
10.989 \\
12.040 \\
14.530 \\
16.453 \\
17.534 \\
17.733 \\
16.987 \\
15.397 \\
13.173\end{array}$ \\
\hline
\end{tabular}

Figure 3.2: Raytracing results for a simulated pellet injection using 10.6 and $119 \mu \mathrm{m}$ wavelengths. Shifts in the chord location due to refraction are listed under Del-R [73]. 
Studies by Irby during the design phase of the Alcator C-Mod system showed that, even for pellet injection, there is little refraction of $\mathrm{CCO}_{2}(10.6 \mu \mathrm{m})$ beam while there is substantial refraction for a $\mathrm{HCN}$ laser $(119 \mu \mathrm{m})$ [73]. Figure 3.2 shows the effect of a pellet on the two wavelengths based on a raytracing code. A pellet is added to a parabolic background density profile with a peak density of $1.0 \times 10^{21} \mathrm{~m}^{-3}$. The column titled "DEL-R" indicates the radial deviation in the path of 11 chords passing through the center of the plasma. The expected phase shift in fringes is also indicated. At the $\mathrm{CO} 2$ wavelength, even for the chord (\#2) passing through the pellet, there is negligible deviation. However, for the $119 \mu \mathrm{m}$ beam the refraction is significant.

\subsection{Design of the Two Color Interferometer for Alcator C-Mod}

\subsubsection{Vibrational Compensation}

In order to accomplish the goal of following pellet ablation in the plasma, minimization of refraction was crucial. $\mathrm{A} \mathrm{CO}_{2}$ wavelength of $10.6 \mu \mathrm{m}$ was chosen as a compromise between signal strength and refraction. However, since vibrational error would then be significant, an additional $\mathrm{HeNe}$ wavelength of $.6328 \mu \mathrm{m}$ was added to correct for any errors. The beams would be run co-axially so that phase shift due to vibrations could be separated from that due to the plasma [71]. Intuitively, since there are two contributions to the total phase shifts, two measurements or equations must be required.

$$
\begin{aligned}
& \Phi_{\mathrm{CO}_{2}, \text { Total }}=\Phi_{\mathrm{CO}_{2} \text {, plasma }}+\Phi_{\mathrm{CO}_{2} \text {, vibration }} \\
& \Phi_{\mathrm{HeNe}, \text { Total }}=\Phi_{\text {HaNe, plasma }}+\Phi_{\text {HeNe,vibration }}
\end{aligned}
$$

where

$$
\Phi_{\mathrm{CO}_{2}, \text { plasma }} \propto \lambda_{\mathrm{CO}_{2}} \int n d l
$$




$$
\begin{aligned}
& \Phi_{\text {HeNe , plasma }} \propto \lambda_{\text {HeNe }} \int n d l \\
& \Phi_{\mathrm{CO}_{2} \text {,vibration }} \propto \frac{\Delta l}{\lambda_{\mathrm{CO}_{2}}} \\
& \Phi_{\text {HeNe ,vibration }} \propto \frac{\Delta l}{\lambda_{\mathrm{HeNe}}}
\end{aligned}
$$

Solving equations (3.4) for the plasma phase shift, the effect of the plasma is

$$
\Phi_{\mathrm{CO}_{2, \text { plasma }}}=\frac{\Phi_{\mathrm{CO}_{2}, \text { Total }}+\alpha \Phi_{\text {HeNe,Total }}}{1-\alpha^{2}}
$$

where $\alpha=\frac{\lambda_{\text {HeNe }}}{\lambda_{\mathrm{CO}_{2}}}$. An additional twist can be added to this compensation scheme. Since there are optical elements that are used prior to the combination of the two wavelengths, there remains an error due to vibrations from these components. Innocente and Martini have suggested that for a heterodyne system, it is possible to create a local reference signal at the point of wavelength combination rather that to use an external reference oscillator [80]. Such a system was tested on Alcator C-Mod using a novel fiber optic coupler. Since any vibration would affect the HeNe system more, a optical HeNe reference signal was created with the coupler. No significant improvement in the compensation to the $\mathrm{CO}_{2}$ beam was observed, indicating that the error due to uncompensated optics in the TCI is negligible.

\subsubsection{Optical Design}

Having determined the appropriate wavelengths for the TCI, the interferometer was designed to facilitate the propagation of an expanded beam. This method has been successfully used on MTX and TEXT [74, 75]. Interferometer systems on JET and DIII- 
D use a more conventional technique of splitting the beam into various chords with beam splitters $[76,77]$. This method allows for easier alignment of the beams since each chord is individually controlled. However, it is more difficult to change chord locations and spatial resolution. With an expanded beam, there is a one to one correspondence between a radial location in the plasma and a location in the returning optical beam. Thus chord location is determined by the position of the detectors in the detector array and spatial resolution is determined by the separation of those detectors. Changes in chord location can be easily accomplished by moving the detector array position. Furthermore, spatial resolution can be improved by taking repeated shots with the detector array in slightly different positions. Figure 3.3 gives a schematic of the optical layout of the TCI $[78,79]$.

The implementation of this expanded beam concept was performed with a commercial geometric optics package, BEAM3 and then verified with Gaussian optics. A basic design was conceived and the exact specifications determined by propagating a geometric cone of rays representing the $\frac{1}{e}$ surface of the beam. The proper divergence and beam size as determined by the laser specifications was used as the initial starting point. Figure 3.4 presents the various resulting beam shapes at different points in the beam path. The final design was then checked and optimized by tracing the beam through the system using the propagation equations for Gaussian optics. The free propagation of the Gaussian waist, $w(z)$, is given by

$$
w(z)^{2}=w_{0}^{2}\left[1+\left(\frac{\lambda z}{\pi w_{0}}\right)^{2}\right] .
$$

The transformation of the waist size and location through focusing optics is

$$
w_{1}^{2}=\left[\frac{1}{w_{0}^{2}}\left(\frac{f-d_{1}}{f}\right)^{2}+\frac{1}{f^{2}}\left(\frac{\pi w_{0}}{\lambda}\right)^{2}\right]^{-1}
$$

and 


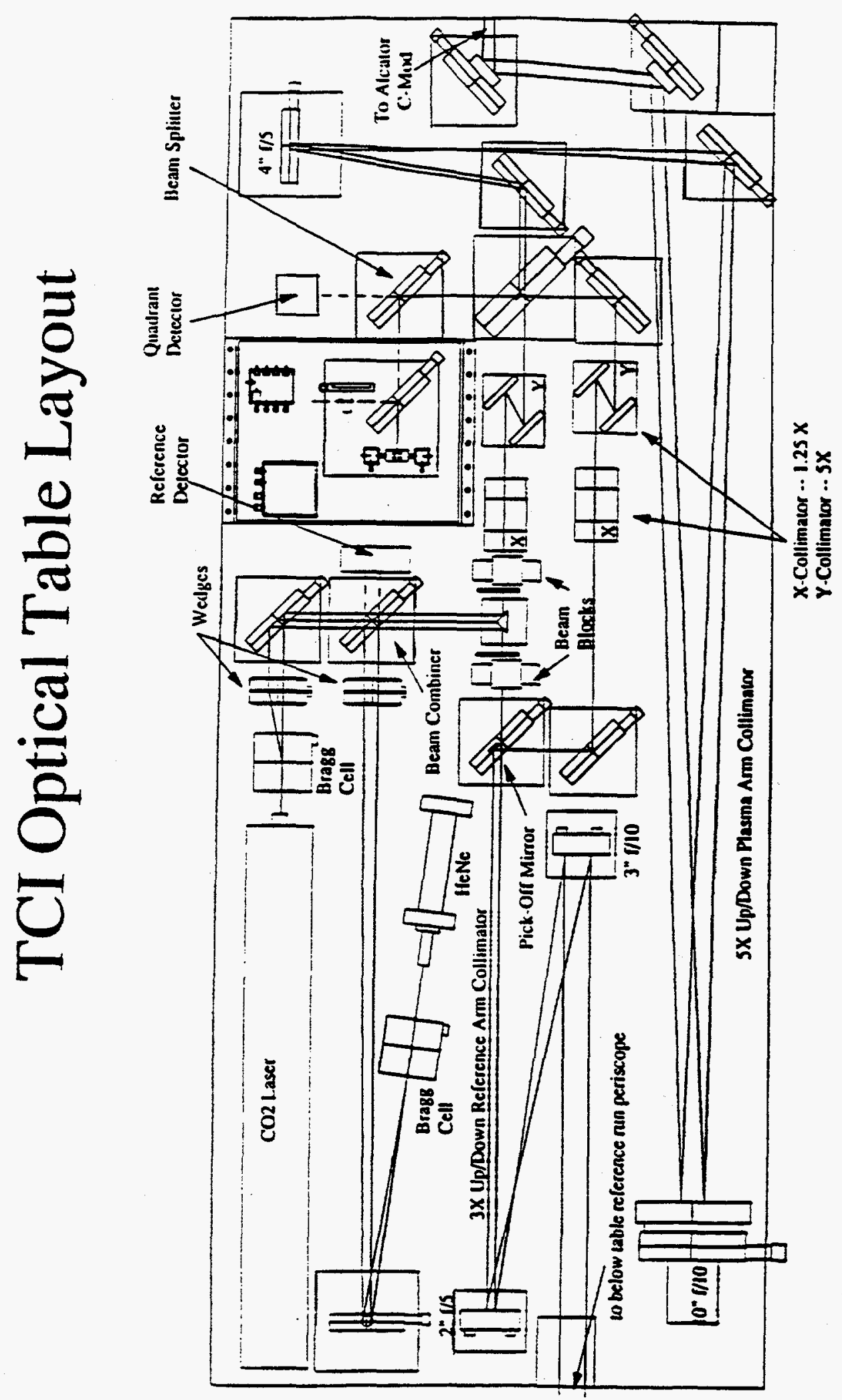

Figure 3.3: Optical layout for the TCI. 


$$
d_{1}=f+\frac{\left(d_{0}-f\right) f^{2}}{\left(d_{0}-f\right)^{2}+\left(\frac{\pi w_{0}^{2}}{\lambda}\right)^{2}}
$$

where 0 refers to the initial values and 1 refers to the transformed values. $f$ is the focal length of the optical component and $\lambda$ is the wavelength of the beam [70].

\subsubsection{Alignment of Co-axial Beams}

In order to minimize the number of uncompensated optical elements, the optics were designed to combine the beams as soon as possible. Both the $\mathrm{CO}_{2}$ and $\mathrm{HeNe}$ beams are passed through acoustic-optic modulators ( Isomet model \# 1207B and \#1201E). These modulators separate the input into a $0^{\text {th }}$ and $1^{\text {st }}$ order beam with a $40 \mathrm{MHz}$ frequency separation. An acoustic-optic modulator or "Bragg cell" is a device that consists of a crystal, typically glass or germanium, through which an acoustic wave is passed transversely to the optical beam. The traveling sound wave acts as a moving diffraction grating which results in the $40 \mathrm{MHz}$ Doppler shift of the first order beam.

The first order beams are then sent through a pair of optical wedges that steer the beams until they are parallel to the $0^{\text {th }}$ order. The $0^{\text {th }}$ and $1^{\text {st }}$ order beams initially have a angle of $77 \mathrm{mrad}$ for the $\mathrm{CO}_{2}$ and $7 \mathrm{mrad}$ for the HeNe between the beams. The two sets of beams are combined on a $\mathrm{ZnSe}$ beam combiner which has the property of transmitting $\mathrm{CO}_{2}$ light and reflecting ( $>90 \%$ ) HeNe radiation. The beams are aligned one on top of each other until both $0^{\text {th }}$ and $1^{\text {st }}$ order beams are traveling co-axially. A mirrored right angle prism then directs the $0^{\text {th }}$ order beams toward the plasma beam optics and the $1^{\text {st }}$ order pair towards the reference arm. 
- Loitia Beaen

\section{Plasma Arm}
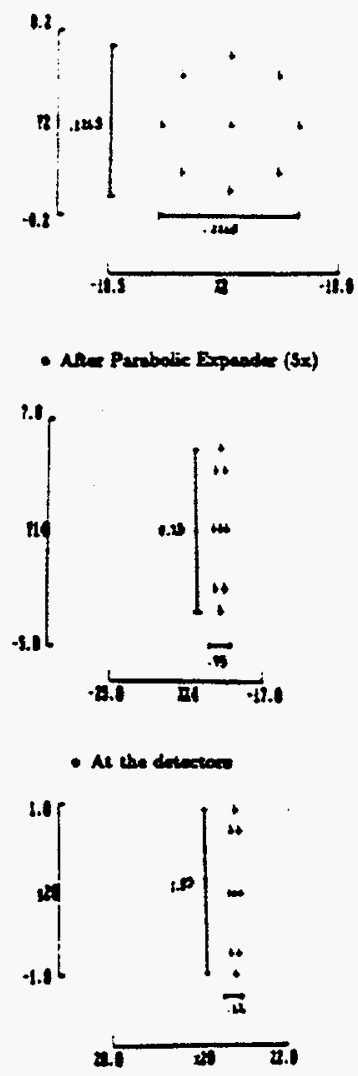

- Ater X-Expader (1.25x)
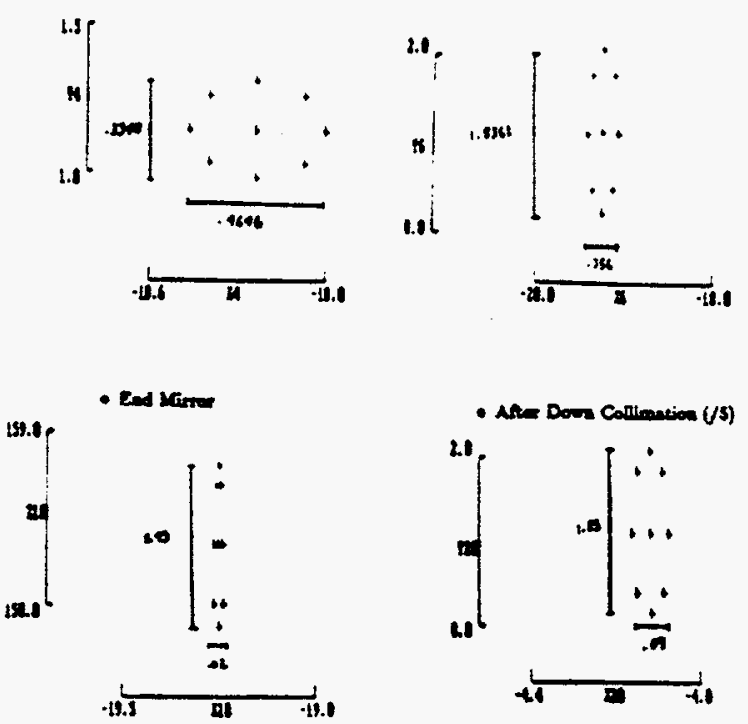

- Nter Y-Expander (5x)

\section{Reference Arm}

- Initiaj Bears
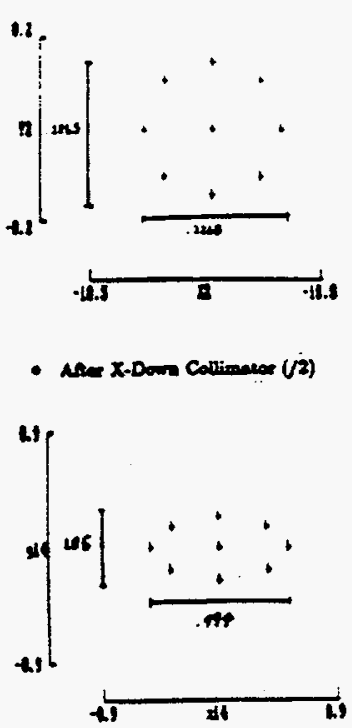
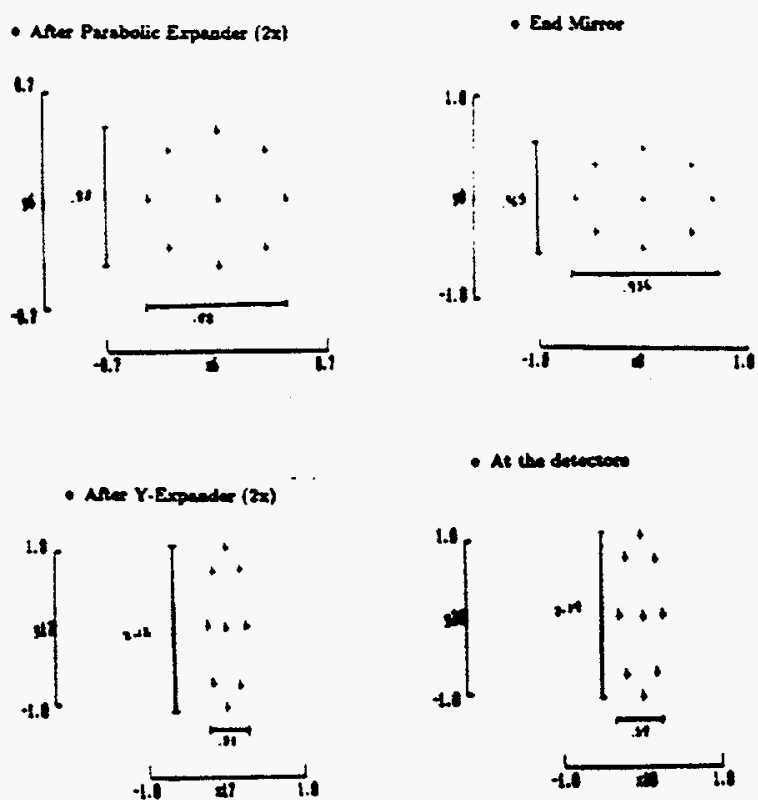


\subsubsection{Plasma Beam Design}

Access to the vacuum vessel and plasma is through tear-shaped vertical ports. Measuring roughly $20 \mathrm{~cm}$ long, with a wide end of $7.0 \mathrm{~cm}$ and a narrow end of $3.2 \mathrm{~cm}$, these ports provide a central view of the plasma in the radial direction. To take advantage of the long port, the interferometer was designed so that the expanded beam is elliptical and makes a double pass through the plasma. Elongation is done by expanding the beam through two sets of cylindrical mirrors and a final set of parabolic mirrors. The first set of mirrors expands the beam horizontally by a factor of $1.25 \mathrm{x}$. The second set stretches the beam vertically by $5 x$ and the final parabola pair provide a $4.76 x$ expansion in both dimensions. (Note the horizontal and vertical directions are referenced to the plane of the optical bench.) The beam is then steered with large rectangular mirrors $(7.6 \times 20.3 \mathrm{~cm})$ through a beam pipe to $45^{\circ}$ mirror under the tokamak.

The beam enters and leaves the vacuum vessel through $\mathrm{ZnSe}$ windows which have anti-reflection coatings at both wavelengths. Transmission for the $\mathrm{CO}_{2}$ beams is greater than $90 \%$ and greater than $95 \%$ for the HeNe beams. A flat mirror housed in a specially constructed G-10 gimbal mount and anchored to the top of the machine igloo directs the beam back through the plasma for a second pass. The beam is given a slight toroidal angle so that after passing back through the parabola pair the outward going and return beams are separated by approximately $1.9 \mathrm{~cm}$. This enables the return beam to strike a $\mathrm{ZnSe}$ beam splitter where it is recombined with the reference beam and directed into the detector box.

\subsubsection{Reference Beam Design}

The reference arm of the Alcator C-Mod TCI incorporates a fully adjustable matching pathlength. The two arms need only be within a coherence length in pathlength ( $\approx 300 \mathrm{~m}$ for the $\mathrm{CO}_{2}$ and $\approx .2 \mathrm{~m}$ for the HeNe), but on the TCI, the reference arm is matched to within $1 \mathrm{~cm}$ of the plasma arm. After being separated from the $0^{\text {th }}$ order 
beams at the prism, the reference beam undergoes a $3 \mathrm{x}$ expansion by a parabolic pair until the beam is roughly $1.88 \mathrm{~cm}$ in diameter. The beam leaving the parabola is adjusted to focus on the end mirror of the reference arm. This ensures that the return beam will be slightly expanding. The beam is directed into a periscope that translates the beam below the optical bench where it is bounced back and forth until the full pathlength is matched. Mirrors below the optic table are mounted on rails to give full adjustability.

The last mirror redirects the beam back along its path but at a slight angle so that after the beam goes through the parabola pair, the return beam is separated from the outgoing beam by $1.9 \mathrm{~cm}$. It strikes a mirror that steers the beam, which is approximately $0.64 \mathrm{~cm}$ in diameter at this point, through $\mathrm{X}$ and $\mathrm{Y}$ collimators which are similar to those in the plasma arm. The beam is expanded into a $4.6 \times 0.64 \mathrm{~cm}$ ellipse that will be combined with the plasma beam on the beam splitter. Once the beams are recombined, they are steered through a small slit in the shielded detector box. Inside, a beam divider, which reflects $\mathrm{HeNe}$ and transmits $\mathrm{CO}_{2}$, directs the beams onto the appropriate detector systems.

\subsection{Electronics Systems}

The TCI electronics is comprised of two main components: The first is the detection systems which generate the $40 \mathrm{MHz}$ beat signals, and the second is the phase demodulator systems which extract the actual phase measurement.

\subsubsection{Detector Systems}

The TCI employs a thermoelectrically cooled Mercury Cadmium Telluride ( $\mathrm{HgCdTe}$ ) detector array to detect the $\mathrm{CO}_{2}$ beams. The array was produced by ElectroOptic Systems ( model \#MCT10TE-010) specifically for Alcator and consists of 10 detectors, each $1 \times 1 \mathrm{~mm}$ in area and mounted on $4 \mathrm{~mm}$ centers. With the expansion 


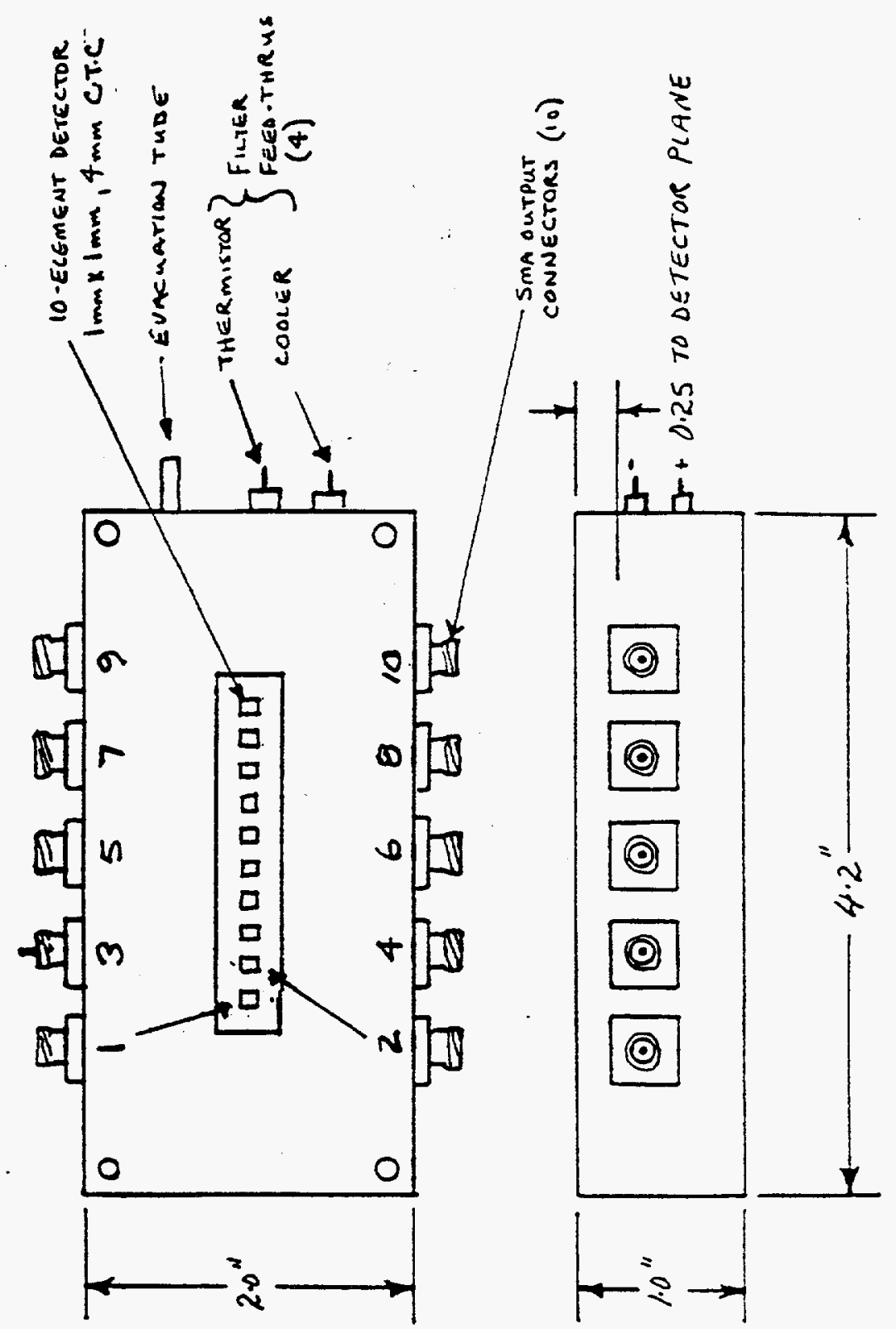

Figure 3.5: Schematic of $\mathrm{HgCdTe}$ detector array. 
ratio of the parabola pair being $4.76 \mathrm{x}$, this gives an effective chord separation through the plasma of $1.8 \mathrm{~cm}$. The detector is thermo-electrically cooled and is inside a nitrogen filled housing fitted with a $\mathrm{ZnSe}$ window. The detectivity of this detector is $2 \times 10^{7}\left(\mathrm{~Hz}^{\frac{1}{2}} \mathrm{~W}^{-1}\right)$ with a responsivity of $200 \mathrm{mV} / \mathrm{W}$ at $10.6 \mu \mathrm{m}$. (see figure 3.5 )

The HeNe beams are detected by avalanche photodiodes produced commercially by RCA Electronics ( model C30950FL). These detectors feature an integrated lightpipe to guide the incoming radiation onto the detection area and built in pre-amplifiers. The units are air cooled with a responsivity of $1.9 \times 10^{5} \mathrm{~V} / \mathrm{W}$. The HeNe detectors are arranged to be aligned with channels $1,4,7$, and 9 of the $\mathrm{CO}_{2}$. Because of space constraints, the detectors can not be mounted directly in the interfering beams. Instead, the signal is guided by short $(\approx 20 \mathrm{~cm})$ lengths of fiber optic cable which couple directly into the light pipes using special alignment adapters.

Both detector systems are housed in an RF shielded box. The beams enter through a narrow $(0.5 \times 7.5 \mathrm{~cm})$ slit with the bias voltages and signals being brought out through a shielded metal conduit to the RF shielded rack.

\subsubsection{Phase Demodulators}

The phase demodulators on the TCI system were designed at MIT based on a Los Alamos National Laboratory design. The demodulators were built by Jorway Corporation (model \# J1819) and are standard single width CAMAC modules with single pin LEMO connector inputs. They employ ECL logic for extremely fast $(\approx n s)$ electronics switching. The basic idea behind the phase demodulation is to have fast counters counting the zero crossings of both $40 \mathrm{Mhz}$ reference and beat signals. One counter increments positively with the other counts down. The sum of the two counts then represents the number of full fringes in the phase shift between the two signals. The divide by 2 counter outputs are used as inputs for an XOR circuit which is then integrated to create an analog 


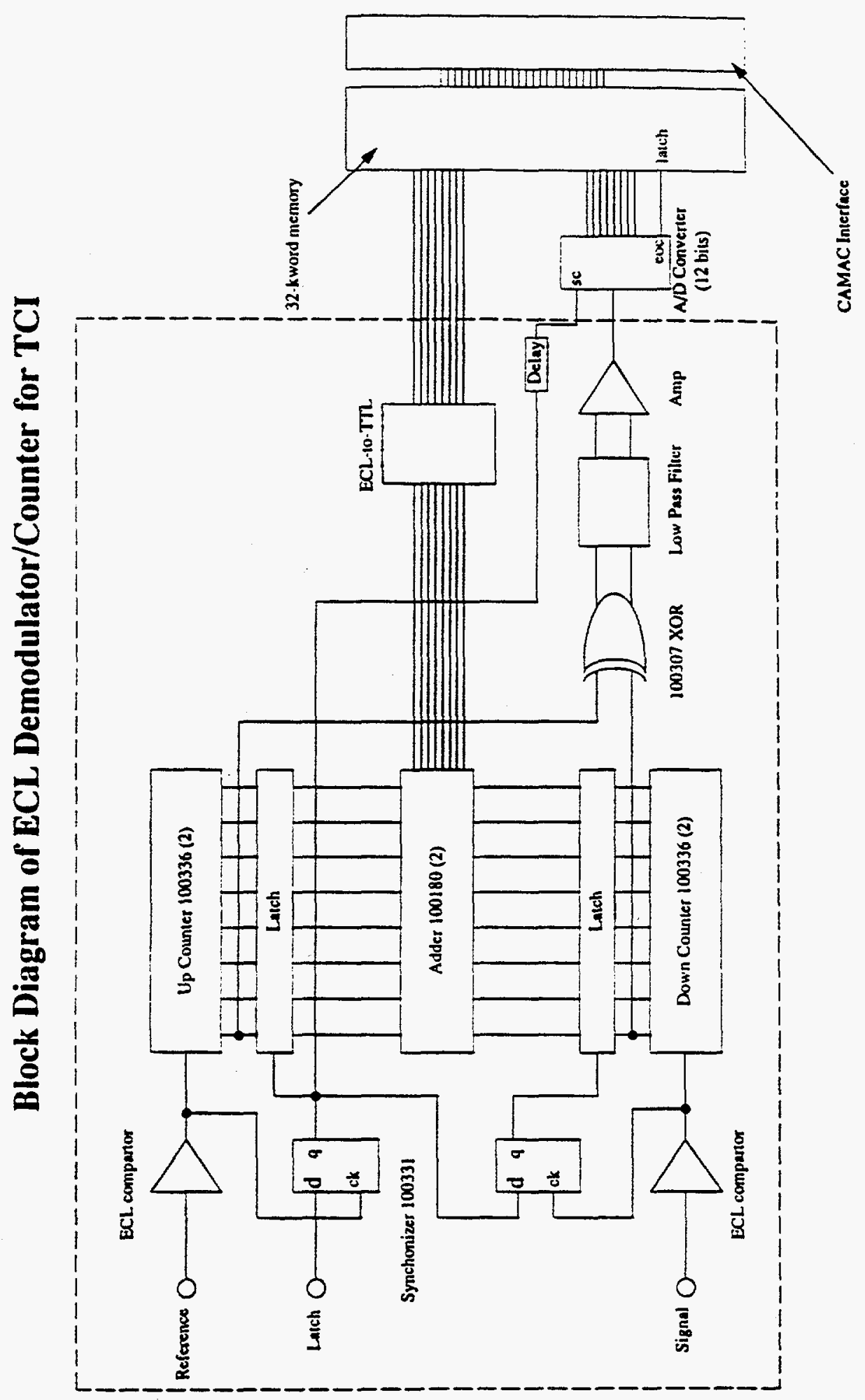

Figure 3.6: Schematic of phase demodulator for TCI. 
voltage signal proportional to the partial phase difference between the two signals.

Combination of the full and partial fringe outputs represents the total phase shift signal. A schematic of the demodulator system is given in figure 3.6.

The demodulators have a range of 256 fringes with a theoretical sensitivity of $0.08^{\circ}$

which corresponds to a line integrated density of $2.33 \times 10^{16} \mathrm{~m}^{-3}$. Each unit has a buffer of $32 \mathrm{~K}$ which provides for $3.2 \mathrm{~s}$ worth of data at $10 \mathrm{kHz}$ digitization. Currently data is taken at a typical rate of $2 \mathrm{kHz}$ and only $8 \mathrm{~K}$ samples for $4 \mathrm{~s}$ of data. The temporal resolution of the demodulator is $1 \mu$ s and is limited by the integrator.

\subsection{Miscellaneous Features}

Because the TCI is located in the cell, beneath the support stand and within close proximity to the vacuum vessel, several special features have been incorporated into the design of the interferometer to accommodate remote operation in high magnetic fields.

\subsubsection{Radiation Sources}

Having chosen a primary wavelength of $10.6 \mu \mathrm{m}$, a commercially produced $\mathrm{CO} 2$ laser was purchased from MPB Technologies ( Model GN802). This is a high power (20 W) continuous wave laser with extremely good frequency stability ( $\leq 1 \mathrm{Mhz}$ variation long term). However, due to the location of the TCI in the cell and the high magnetic fields associated with that position ( $500-1000$ gauss), it was necessary to modify the laser. (see Figure 3.7) The magnetostrictive Invar rods supporting the laser cavity were replaced by custom made carbon extruded rods. Furthermore, to ensure frequency stability of better than $1 \mathrm{MHz}$, the electro-magnetic translator (EMT) that regulated the cavity length was replaced by a PZT modulator that would not be affected by magnetic fields. The PZT uses a dithering feedback system in which the voltage to the PZT is slightly modulated at $512 \mathrm{~Hz}$. As the cavity length changes slightly, the output power of 


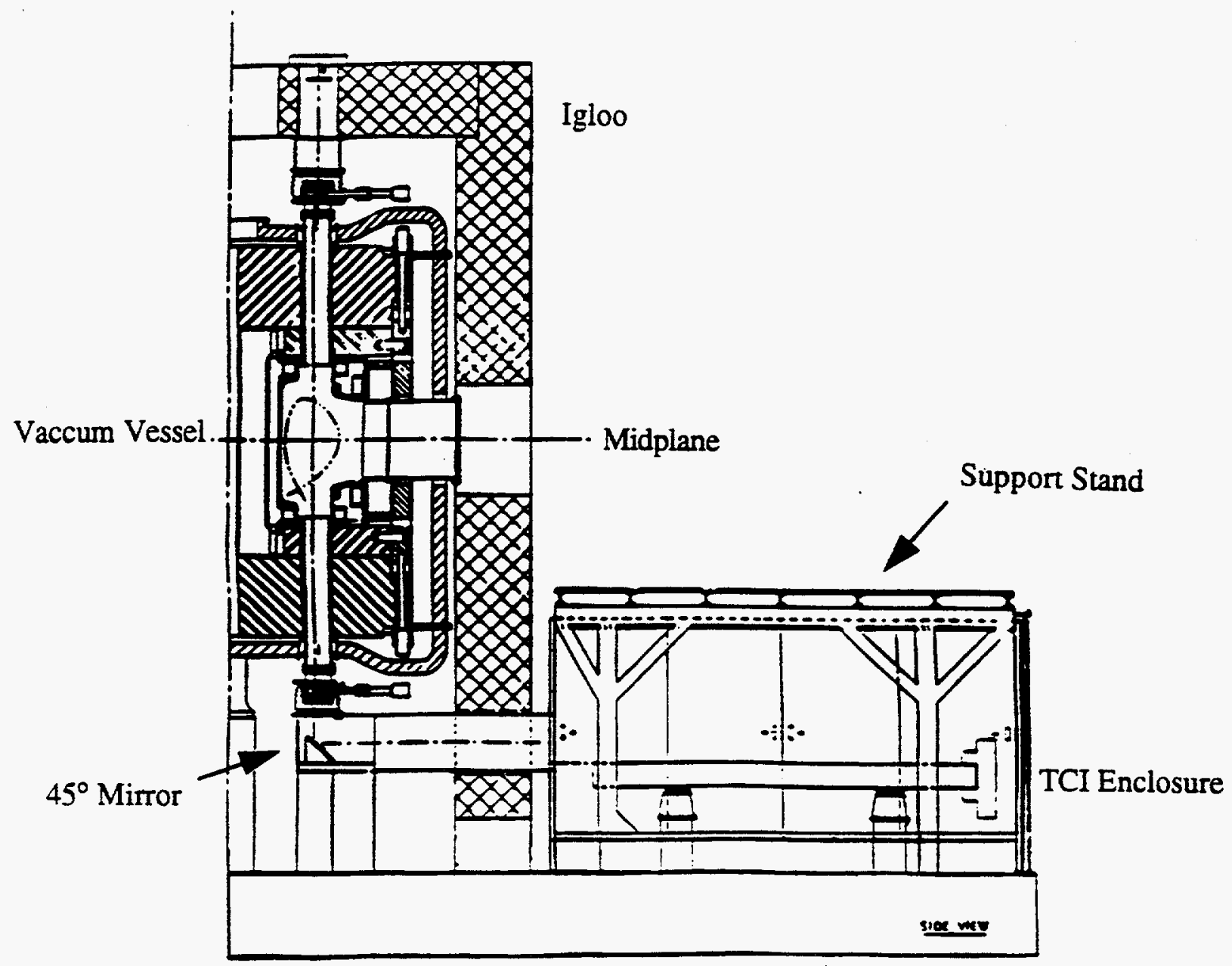

Figure 3.7: Location of TCI system relative to the vacuum vessel. 
the laser is monitored and fed into a lock-in amplifier that generates a feedback signal to the PZT. In this way, the laser is kept tuned to the P20 $(10.6 \mu \mathrm{m})$ line.

\subsubsection{Remote Operation}

Limited access to the TCI during operation of the tokamak requires remote control of the system. Thus, both the $45^{\circ}$ mirror and the top igloo mirror can be operated remotely as well as the end mirror on the reference beam. A CCD TV camera is used to monitor changes in the beam position. An Allen Bradley PLC has also been programmed to operated the gate valves and beam blocks. Vacuum pressures at the $\mathrm{ZnSe}$ windows and cooling water flows through the laser and Bragg cell are all monitored and interlocked.

\subsubsection{Feedback Control}

The alignment of any interferometer is crucial to successful operation. Given the proximity of the interferometer to Alcator C-Mod, the TCI is subject to vibrations and contractions of the tokamak vessel during shots and to thermal effects due to cooling. Furthermore, access to the system is restricted during tokamak operations To ensure continuous proper alignment, a feedback system was developed. The reference beam is assumed to remain fixed since all the optical components of that beam are attached to the main optical bench. Ten percent of the returned HeNe plasma beam is focused onto a UDT quadrant detector. The detector measures both the direction and magnitude of beam position changes and feedbacks to the end mirror on top of the igloo. The system can respond on a $1 \mathrm{~ms}$ timescale which is more than sufficient for maintaining long term stability. 


\subsubsection{Acoustic and Thermal Insulation}

There are significant thermal gradients in the cell due to the cooling of the tokamak magnetic coils. Furthermore, the lower mirror mount is subject to flows of liquid and gaseous $\mathrm{N}_{2}$. To insulate the $\mathrm{TCI}$, a complete enclosure of fire retardent thermai insulation with aluminum backing was constructed. The enclosure has sliding doors to allow access to the optic bench. To prevent dust from entering the room, a pair of filtered fans maintain positive pressure inside.

The beam pipe leading to the lower $45^{\circ}$ mirror mount is also fully insulated and the mount itself is housed within an insulated G-10 box. The mirror is supported by carbon rods and the box is weighted with several hundred pounds of sand to prevent accidental bumping and shifting of mirror position. A G-10 and fibercast interface with the $\mathrm{ZnSe}$ window flange on the vertical port provides protection for the lower mirror mount from condensation and the $\mathrm{LN}_{2}$ cooling. A similar fibercast and $\mathrm{G}-10$ pipe guides the beam from the upper window flange, through the igloo cover and onto the upper mirror mount. The mirror mount is bolted to a heavy (2") G-10 plate which itself is attached to the igloo cover for stability. The entire assembly is covered with the foam insulation with a plastic backing. Both upper and lower mirror assemblies are purged by dry nitrogen to prevent dirt buildup and condensation.

\subsubsection{Position Scanner}

It is necessary to be able to determine the chord locations in an expanded beam configuration. As mentioned each position in the beam corresponds to a single major radial location. To calibrate the chord location, a scanning beam block has been installed. Locally or remotely, this block can be activated to scan and block the beam. The data system is set to simultaneously measure the chordal signal levels. The time that the signal drops can be correlated with the scan position which can then be related to a major radial location with the appropriate calibration. 


\section{Chapter 4}

\section{Profile Inversions}

Having previously described the interferometer and data acquisition systems used to measure the phase shifts, this section will give the details of the data analysis used to obtain useful information from these density measurements. The analysis can be separated into two stages. The first task is to invert line-integrated data into density profiles. This methodology will be presented in this chapter. The second task will be to extract the diffusion coefficients which will be described in the following chapter.

\subsection{Background}

There have been various techniques developed to invert line integrated data into density profiles $[81,82,83,84,85]$. The most straight forward of these is the Abel inversion [71]. In this method, the inversion is performed through a pair of transform integrals:

$$
\begin{aligned}
& N_{L}(r)=2 \int_{r}^{a} n(\rho) \frac{d \rho}{\sqrt{\left(\rho^{2}-r^{2}\right)}} \\
& n(\rho)=\frac{-1}{\pi} \int_{\rho}^{a} \frac{d\left(N_{L}(r)\right)}{d r} \frac{d r}{\sqrt{\left(r^{2}-\rho^{2}\right)}} .
\end{aligned}
$$

$\mathrm{N}_{\mathrm{L}}$ is the line integrated density; $\mathrm{n}$ is the density; $\mathrm{r}$ is the radial location of the chord; $\mathrm{a}$ is the minor radius; and $\rho$ is the flux co-ordinate. This method is most easily applicable to systems with a circular geometry or those that are easily transformable to a circular geometry (e.g. elliptical flux surfaces). The technique has been extended by Yasutomo to systems with density asymmetries [84]. 
In more complicated co-ordinates and especially for shaped and diverted plasmas, no analytic transform exists and numerical techniques must be used. Several techniques have been developed that employ a method intuitively referred to as "peeling an onion" $[82,85]$. In this methodology, phase measurements on the outside of the plasma are used to unfold the outer density. This contribution is then subtracted from all other phase signals. The next layer is then unfolded until the center is reached. In this way, if measurements exist on both sides of the plasma center, in/out asymmetries can be taken into account [86]. Error analysis has shown that the largest uncertainties for such a method come from spline interpolation and errors in the magnetic geometry. Errors in the geometry are second order effects. Spline errors can be significant, but Park has showed that they are usually localized and spatially decay away quickly [86].

\subsection{Inversion Analysis for Alcator C-Mod}

For Alcator C-Mod, the technique used for density profile inversions is a SVD matrix inversion analysis [87]. Inside the last closed flux surface (LCFS), where the plasma is confined, parallel transport is much faster than radial cross-field transport. As a result, it is a reasonable assumption that density and temperature equilibrate quickly on a flux surface and, therefore, are flux quantities. In the SVD technique, this assumption is extended to the scrapeoff layer; poloidal asymmetries are ignored and density is assumed to be constant on flux surfaces beyond the LCFS. Measurements made by the divertor and reciprocating probes indicate that under certain conditions, the pressure, density and temperature do remain constant on the flux surface. This is illustrated in fig. 4.1 where for $\mathrm{n}_{\mathrm{e}} / \mathrm{I}_{\mathrm{p}}<1.5 \times 10^{6} \mathrm{~m}^{-3} / \mathrm{A}$, the density at the divertor probe and the upstream density as measured by the reciprocating probe are equal [55]. However, when a MARFE or detachment exists, there may be poloidal asymmetries and density gradients along a flux line in the scrape-off layer [112]. Nevertheless, in order to be able to perform an inversion of the chordal data with the SVD technique, the assumption that the density is a flux 


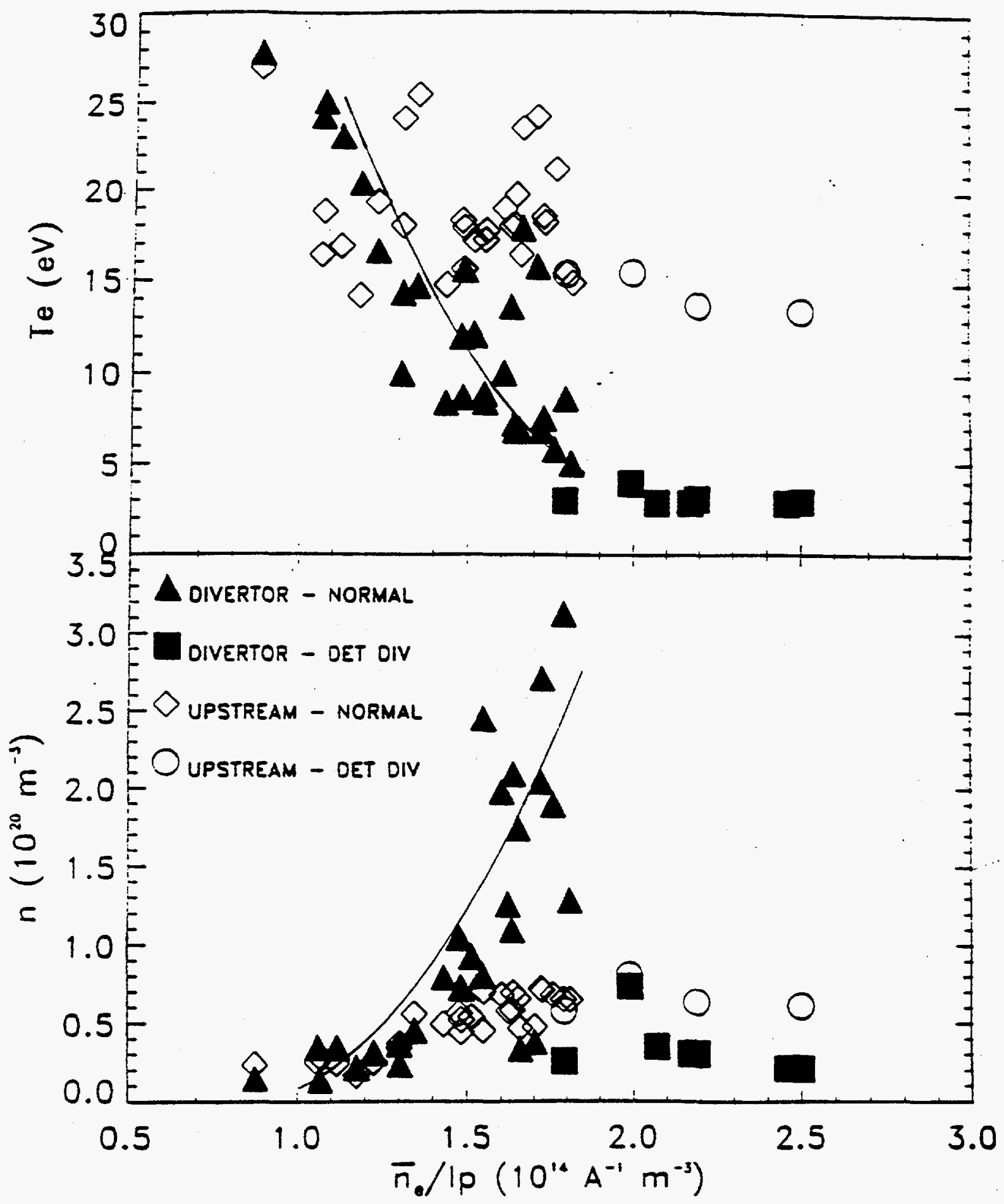

Figure 4.1: Comparison of ne and Te measurements from the divertor probes and reciprocating probe. 
quantity outside the LCFS must be made. It will be shown later that this assumption is not crucial for two reasons: 1) the scrapeoff-layer is a small contribution to the overall phase measurement and 2) the analysis is insensitive to the details of the profile shape outside the LCFS; only the magnitude of the density at the LCFS is important.

The basic philosophy behind the SVD inversion is to invert the pathlength matrix. The flux geometries are measured with magnetics diagnostics and reconstructed with an EFIT or FFIT analysis [88]. Flux surfaces are then defined at 16 locations in psi, $\Psi$, the variable denoting the flux surfaces. Typically, there is at least one flux surface defined to cover the scrape-off layer. The analysis is performed in $\Psi$ coordinates in order to allow for transiational movements of the plasma. By performing the analysis in flux coordinates, the reference frame is not static so that no explicit term is necessary to keep track of the position of the plasma center. The density is tied to a flux surface and moves with that surface. The path length contribution, $\mathrm{dl}$, to each chord is calculated from each of these flux surfaces by determining the intersection point of each chord with the flux surfaces as shown in fig. 4.2. Thus the phase measurements can be represented by the equation:

$$
\tilde{A} n_{\psi}=\Phi
$$

where $\tilde{A}$ represents the pathlength matrix, $\mathrm{n}$ is the vector of densities on the flux surfaces and $\Phi$ is the vector of measured phase shifts. Through the SVD inversion, the inverse of the $\tilde{A}$ matrix is constructed and multiplied through (4.3) to obtain

$$
n_{\psi}=\tilde{A}^{-1} \Phi
$$




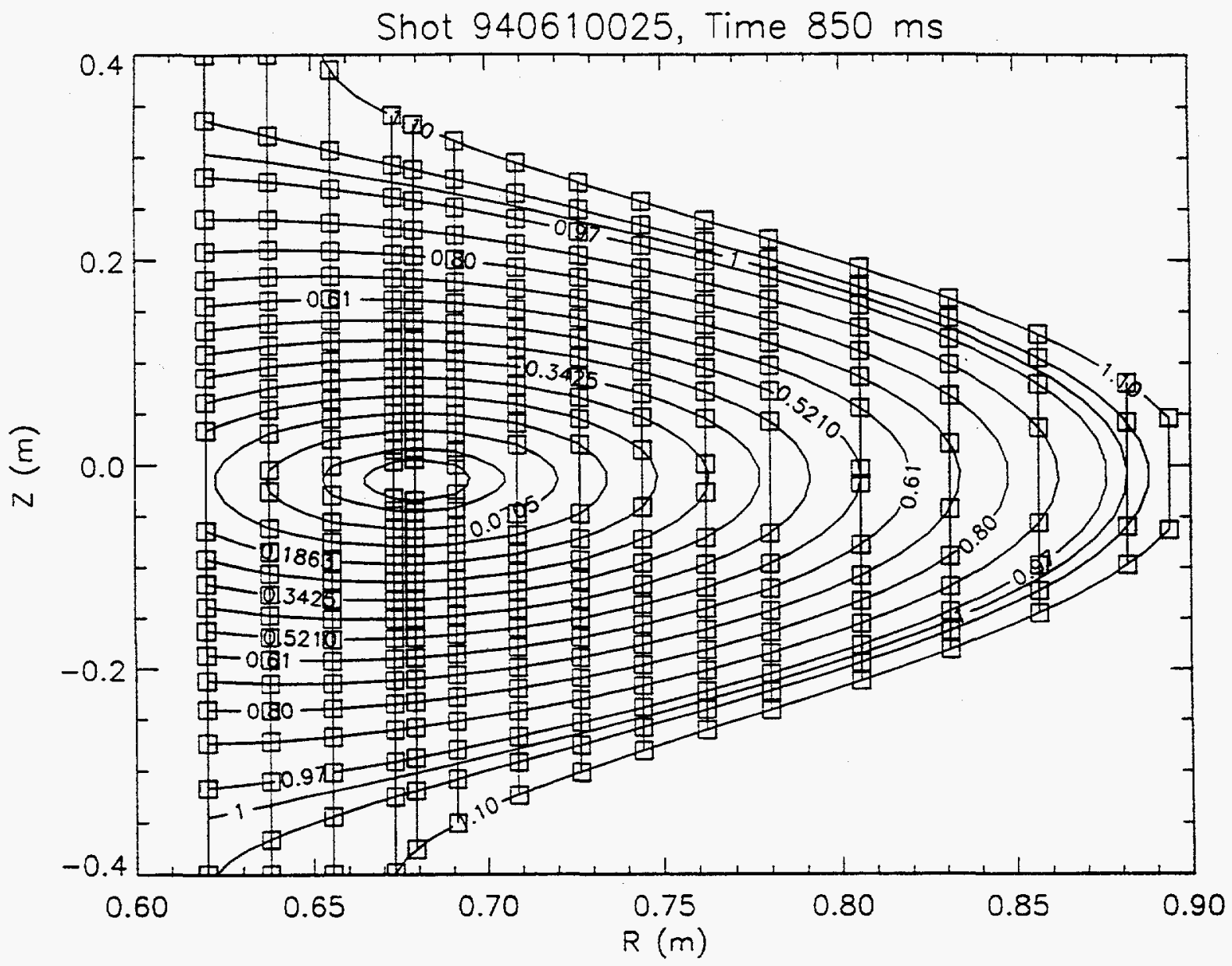

Figure 4.2: Intersection of the chords with the flux surfaces. 
The construction of the pathlength matrix is straightforward. The only complication that can arise is when the plasma is diverted. Care must be taken to account for plasma in the divertor. Furthermore, the intersection of the chords with flux surfaces in the divertor region can be confusing since the flux surface may be tangent to the chord. In these cases, the pathlength contribution is unphysically long and must be truncated.

\subsubsection{Boundary Conditions}

In addition to the path length matrix equation, boundary and smoothness conditions can be imposed on the solution. The most obvious boundary conditions are that at the magnetic axis the density gradient vanishes and at the plasma edge the density vanishes, namely

$$
\begin{aligned}
& \left.\frac{d n}{d r}\right|_{r=0}=0, \\
& n(a)=0 .
\end{aligned}
$$

Here $a$ is the minor radius that defines the edge of the plasma, i.e. the limiter radius or several e-folding lengths beyond the LCFS.

Similarly a smoothness condition requiring the minimization of the second derivative or the quantity, $\mathrm{S}$,

$$
S=\min \left(\left.\sum \frac{d^{2} n}{d r^{2}}\right|_{\substack{\text { Evaluated } \\ \text { on each } \\ \text { flux surface }}}\right)
$$

can be imposed on the system. The relative weight of these boundary and smoothness equations, $\varepsilon$, can be adjusted. This will be discussed in the next section. With the addition 
of boundary constraints and smoothness criteria, the system is now overdetermined and can be inverted with the SVD methodology. The resulting inversion analysis is equivalent to that of a least squares fit for such overdetermined systems and the quantity, $Q$, to be minimized is given by [74],

$$
Q=\left|\tilde{A} n_{\psi}-\Phi\right|+\varepsilon S
$$

\subsubsection{Determination of Smoothing Weight}

The smoothing equations are essential to the inversion problem. Without these extra conditions the problem would be underdetermined. Thus, it is crucial that the appropriate weighting, $\varepsilon$, be placed on the smoothness of the solution.

Epsilon multiplies the smoothing equations in eqn 4.7 and there is a clear tradeoff between fitting the data and the smoothness of the solution. As $\varepsilon$ increases from zero the smoothness of the solution increases until for high values of $\varepsilon$ the fit is a straight line. In order to choose the proper $\varepsilon$, several schemes were considered. In all cases, it was constrained that the inverted profile match the desired edge density to within $10 \%$. Only in this way, would a sensitivity study with the edge density be meaningful. The most straightforward approach to selecting $\varepsilon$ is to choose the value that minimizes the residuals of the entire SVD problem, both chord measurements and smoothness constraints. This would be equivalent to a standard least-squares problem. However, the smoothness represents a desired attribute as opposed to a real measured quantity. Thus, a bias would be introduced if the smoothness equations were weighted on an equal basis to the phase measurements. The solution should fit the phase measurements and the smoothness of the profile should be regarded as a secondary consideration. Similarly, a minimization of the residuals of the chord measurements alone would bias the fit to low $\varepsilon$ and result in a profile with too much structure which is clearly unphysical. 
A manual selection of $\varepsilon$ at several trial timesteps was also examined. The selection was based on the following criteria. The largest possible $\varepsilon$ was chosen such that at the test timeslices the profile fit the measured phases to within three degrees on average. This is equivalent to the average measured subtraction error. The maximum error on any chord was less than seven degrees and the desired edge density was matched. This method yielded limited success. It was found that a factor of two change in $\varepsilon$ can occur over several timesteps so that there is high sensitivity of this method to the selection of trial timeslices themselves. The inversion was quite reasonable for any single timestep but the chosen values of $\varepsilon$ were not consistent in time.

Choosing an individual $\varepsilon$ for each timeslice was attempted under the condition that the reduced $\chi^{2}$ of the fit was equal to one. This scheme was rejected based on two observations. Chi-squared should follow a $\chi^{2}$ distribution rather than having a constant value of 1. Also, in the SVD technique each timeslice is inverted as an independent problem. There is no correlation between timeslices except through the chord measurements and the smoothing parameter. However, for similar sets of data, similar inversions are expected. By selecting independent $\varepsilon$ at each time, the time correlation of the inverted profiles would be further weakened. In an actual test, it was observed that the variation in the inverted profiles was indeed more pronounced. The inversion was fitting the random noise in the data.

The selected scheme incorporates features from all these methods. A single $\varepsilon$ is chosen for the period of interest, $600-1000 \mathrm{~ms}$ It is selected by scanning through the range of $\varepsilon$ and computing the time dependent $\chi^{2}$ for each value. Appropriate limits are determined for the $95 \%$ confidence level of $\chi^{2}$ using the exact number of degrees of freedom. This number is typically around eight since the SVD solution typically has a rank of 2. A value of $\varepsilon$ is considered to be acceptable if the $\chi^{2}(t)$ of the fit is within the limits of the confidence interval. The best value of $\varepsilon$ can then be chosen from the intersection of this set of $\varepsilon$ 's and the set of $\varepsilon$ 's that give a good fit to the edge density. It 
can be chosen as the largest value of the intersection set or the average value. Thus, the result is a set of inverted profiles that statistically fit the phase measurements according to a $\chi^{2}$ distribution. The solution is also sufficiently smooth since the smoothing weight can be biased towards larger values in the set of allowable $\varepsilon$. Finally, there remains some correlation between timeslices through a constant value of smoothing. Thus, this technique resolved the issues mentioned above and was applied to all gas modulated shots.

Studies with this analysis revealed several interesting trends. When $\varepsilon$ is small, the system is effectively underdetermined. As a result, very small values of $\chi^{2}$ can be measured; in fact, they are so low that they are statistically unlikely. As $\varepsilon$ increases, $\chi^{2}(t)$ increases and lies within the limits of the $95 \%$ confidence level. As $\varepsilon$ increases further, $\varepsilon \geq$ 2 , the fits start to worsen and $\chi^{2}$ again exceeds the statistical limits. As expected, the fit to the desired edge density also worsens as $\varepsilon$ increases. The fractional error monotonically increases and for certain values of the edge density, errors of greater than $50 \%$ have been observed for large values of $\varepsilon$. In figure 4.3 , a typical example is shown. The top plot is the fractional error of the inverted edge density and the lower plot represents the fraction of $\chi^{2}$ outside of the $95 \%$ confidence level. For acceptable $\varepsilon$ this fraction must be $<.05$. The range of acceptable $\varepsilon$ is between 0.7 and 1.6. A value of 1.1 would represent the minimum in the $\chi^{2}$ error.

The range of $\varepsilon$ for which the fit to the edge value and the chords are satisfied increases and then decreases as a function of the desired edge density. This is expected since for the true value of the edge density the chord measurements should fit easily. Slightly incorrect edge values can also be fit by adjusting the smoothness parameter. However, when the edge density required increases or decreases beyond a certain point, no reasonable value of smoothing will allow a fit to the chords and the density simultaneously. An $\varepsilon$ of zero would fit the phase measurements but the $\chi^{2}$ of the resulting profile would be very small and statistically significant. The profile would contain too many bumps to be plausible. 

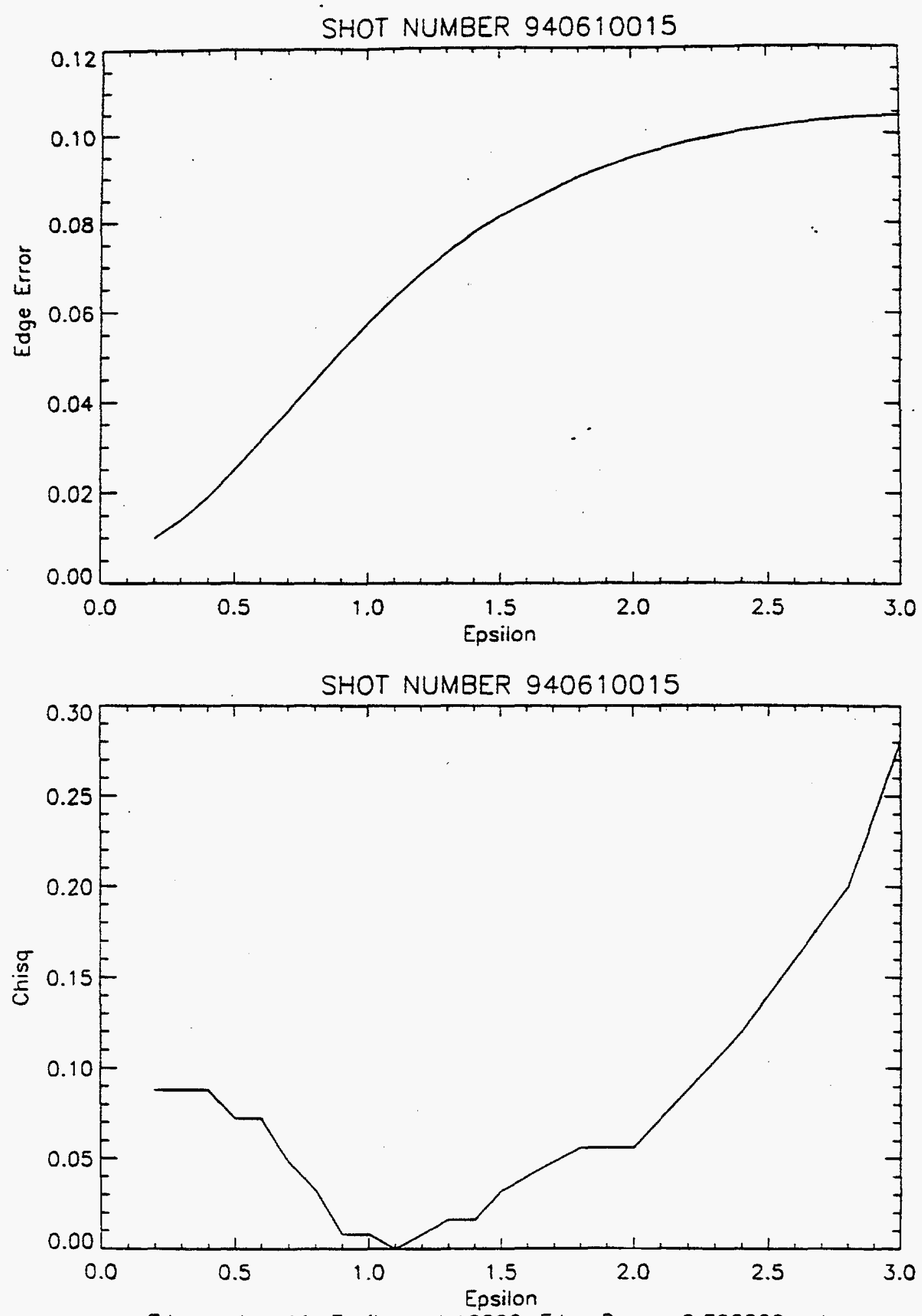

Figure 4.3: Typical result from the $\varepsilon$ analysis. Error in the edge density must be $<0.1$ and the chisq error must be $<0.05$. 
Thus, the analysis of the appropriate $\varepsilon$ yields additional information about the edge density. For certain values of edge density, the chord measurements can not be fit regardless of what smoothness parameter is used. Table 6.1 lists the best values of $\mathrm{n}_{\text {edge }}$ as determined by this $\varepsilon$ analysis.

\subsection{Edge Model: General Methodology}

Because the view that the TCI covers on Alcator C-Mod is restricted to the central plasma $(r / a<0.5)$, edge measurements or at least an edge model are needed to uniquely invert the profile. In the future, the microwave reflectometer and Thomson Scattering (core and edge) systems can supply data about both the edge density and its gradient. Currently such measurements are not available and only the measurements from the divertor and fast scanning probes or limiter probes can be incorporated into the inversion. Thus, several methods have been developed and implemented in order to estimate the edge density. In the SVD inversion matrix, two flux surfaces have been defined. One covers the LCFS and the second spans the scrapeoff layer. By appropriately defining the densities at these two surfaces, both the edge density and gradient information can be incorporated into the inversion.

\subsubsection{Scalings from Fast Scanning Probes.}

Studies of the edge have been performed with the fast scanning probe for both attached and detached diverted plasmas. The density profiles have been measured up to the LCFS for a range of plasma currents and central densities. Figure 4.4 gives an example of the density profile within the scrapeoff layer. $\rho$ is the distance from the LCFS. A database has been built up that allows scaling of the density at the LCFS and the scrapeoff e-folding length with the plasma current, Ip, and the line averaged central density, $\bar{n}_{e}$. From figure $4.5 \mathrm{a}$ and $\mathrm{b}$, the scalings are 


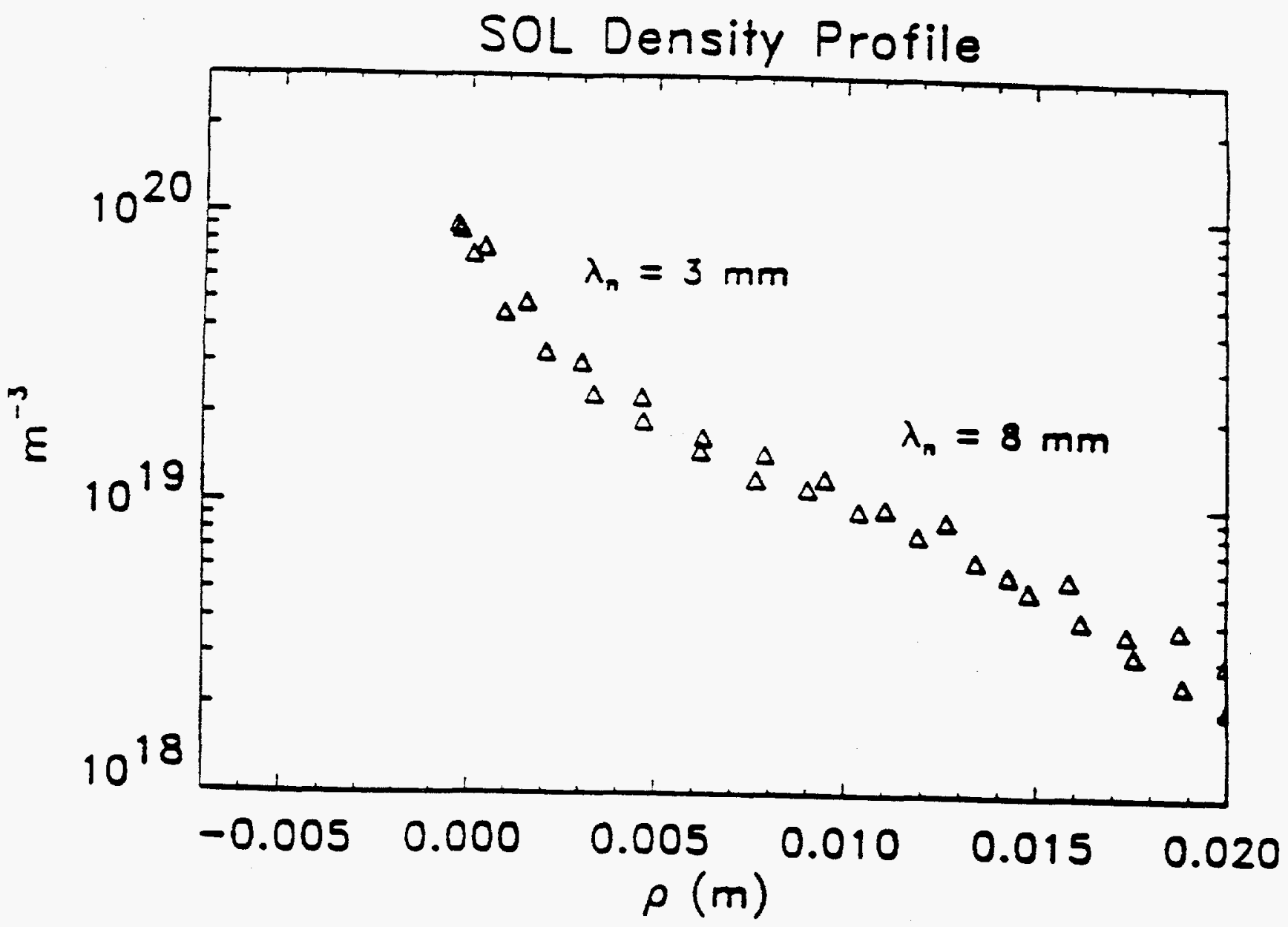

Figure 4.4: Measurement of the density profile in the scrapeoff layer from the reciprocating probe. 

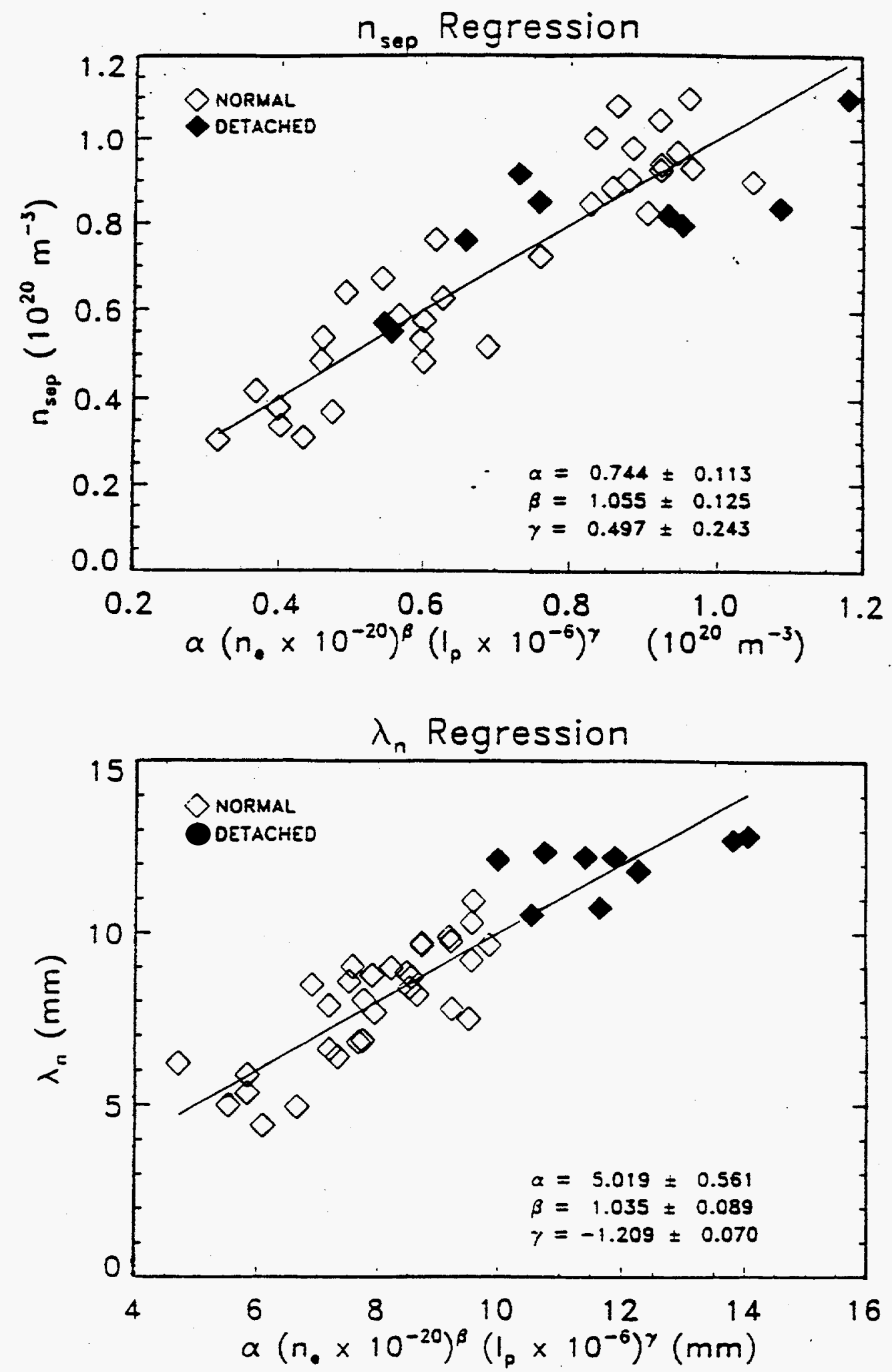

Figure 4.5: Scaling of the edge density and the scrapeoff length with central density and plasma current using the reciprocating probe. 


$$
\begin{aligned}
& n_{\text {lcfs }}=0.744\left(\bar{n}_{e} \times 10^{20}\right)^{1.055}\left(I_{p} \times 10^{-6}\right)^{.497} \quad\left(10^{20} \mathrm{~m}^{-3}\right), \\
& \lambda_{\text {lcfs }}=5.019\left(\bar{n}_{e} \times 10^{20}\right)^{1.035}\left(I_{p} \times 10^{-6}\right)^{-1.209} \quad(\mathrm{~mm}),
\end{aligned}
$$

where $n_{l c f s}$ and $\lambda_{\mathrm{lcfs}}$ are the density and scrapeoff e-folding length at the LCFS [90]. Through these scalings, the edge profile can be defined in terms of central plasma parameters. This provides edge densities on the same timescale as the chordal measurements (i.e. typically $0.5 \mathrm{~ms}$ ) which is significantly better than most probe diagnostics.

Changes in the edge density have a finite propagation time into the central plasma, typically on the order of $10 \mathrm{~ms}$. Thus, if central plasma parameters are used to determine the edge density, the time histories of the edge can be distorted and delayed. Fast changes in the edge will appear to be slower due to the effect of the bulk plasma. In general, the quantities of interest are some functional of the inverted density profile (e.g. total number of particles) and this distortion in the time history will not pose a serious problem. In fact figure 4.6 shows the total particle number for two inversions in which the edge density values differed by a factor of 4 . Less than a $10 \%$ difference can be seen. However, in transport analysis where the information about the transport coefficients is contained in the time history of the profiles, greater accuracy in the time histories of the edge density is required. Thus, a second model was developed with data from the probe measurements.

\subsubsection{Probe Measurements}

It was observed that the plasma did not detach above the nose of the divertor and that the pressure remained a flux quantity for probes 8,9 and 10 . As a result, a continuous measurement of the plasma pressure can be made at the divertor probe and extrapolated back to the midplane along the field lines in the scrapeoff layer that end on these probes. Figure 4.1 shows, as a function of $\bar{n}_{e} / I_{\mathrm{p}}$, the regime in which pressure 


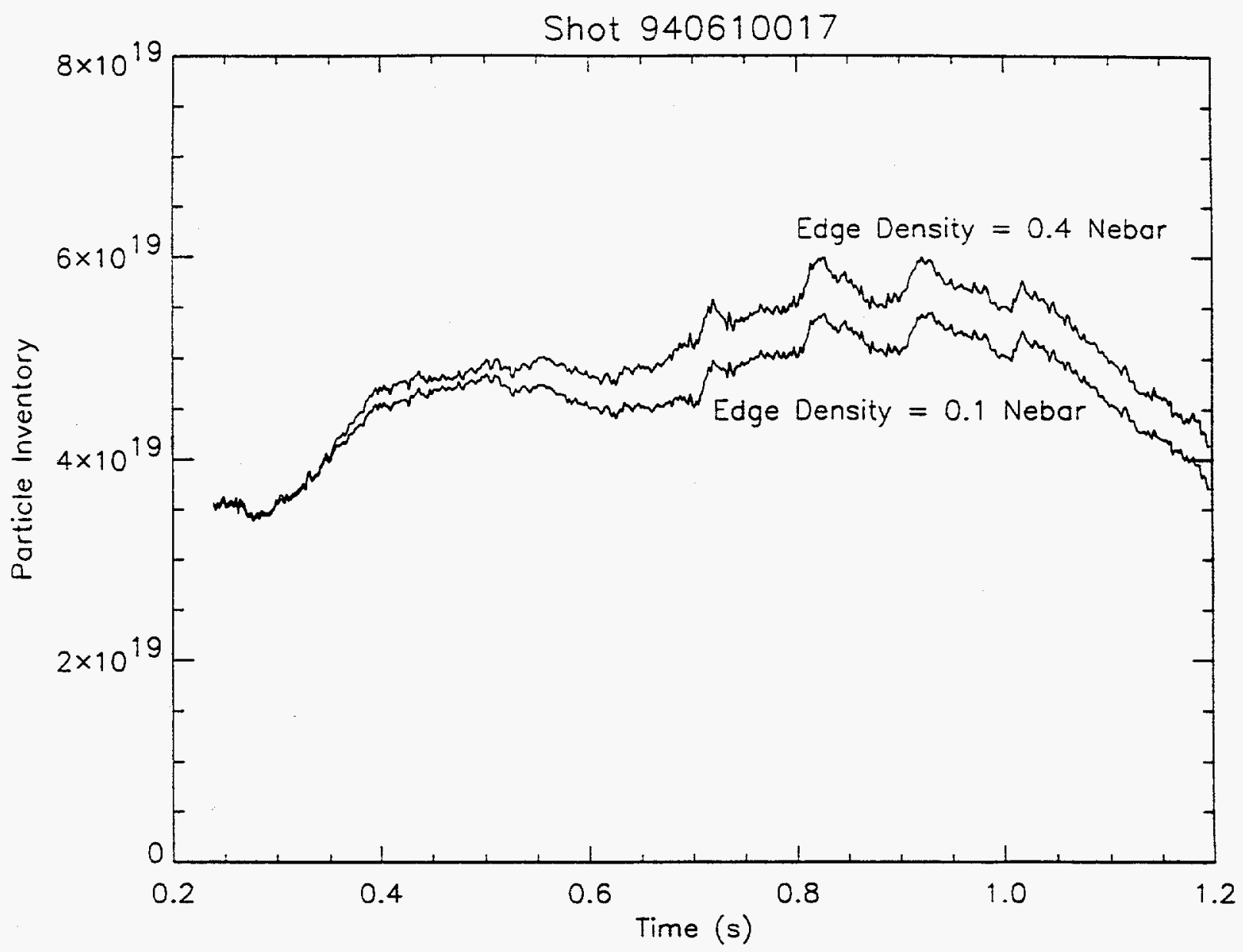

Figure 4.6: Comparison of the particle inventory as calculated from the inversion using two edge densities. 
consistency holds and the gradients along the field lines are small enough so that it is possible to extrapolate the probe measurements at probe 7 back to the midplane. For $\bar{n}_{e} / \pi_{\mathrm{p}}<1.5$, there is clearly little difference between the measurements near the divertor and those near the LCFS. Knowledge of the upstream temperature would allow the determination of the upstream density. The standard edge plasma heat transport model shows that the largest dependence of the upstream temperature is on the heat flux to the scrapeoff layer [89]. By power balance arguments,

$$
T_{\text {upstream }} \propto\left(\frac{P_{O H}-P_{R a d}}{A_{S e p}}\right)^{\frac{2}{7}}
$$

This dependence indicates that large changes in the power flux to the scrapeoff layer are required for significant changes in the upstream temperature During the gas modulation, the plasma current was constant to within $2 \%$ and the radiated power as measured by the bolometer arrays was constant to within $15 \%$. Such small changes in the power flows suggest that the upstream temperature did not change significantly during the gas modulation. By using the fast scanning probe during the gas modulated shots, the upstream temperature was measured at the time of the probe injection. It is reasonable to assume that this temperature remained constant during the current flattop. The upstream density can then be determined through

$$
n_{\text {upstream }}(t)=\frac{p(t)}{T_{\text {upstream }}(t)}
$$

There are now two continuous and independent measurements of the density profile in the scrapeoff layer: 1) the extrapolation of the divertor probe to the midplane 
and 2) the density measurement from the limiter probes. It is possible to determine the exponential curve through these two points. By solving

$$
n(\rho)=n_{L C F S} e^{\frac{\rho}{\lambda\left(\bar{n}_{e}, I_{p}\right)}},
$$

for the free parameters nLCFS and $\lambda$ at each timeslice, the density and slope at the LCFS can be determined as a function of time.

The biggest limitation to this model are that the points used to determine the exponential profile may be up to $0.5 \mathrm{~cm}$ from the LCFS. This introduces error through the extrapolation of the density and gradient to the separatrix. Thus, the error bars on the edge density can be up to $50 \%$ of the value. It will be shown in chapter 6 that even with these error bars, the value from this probe edge model agrees well with other estimates of the edge density.

To summarize, this second edge model determines the density at the LCFS through the following procedure:

1) Determine the pressure at divertor probe 9. Check that the measured pressure is consistent with the pressure measured by the fast scanning probe.

2) Use the fast scanning probe to measure the upstream temperature. Check the ohmic power and the bolometer measurements of the radiated power. If these are relatively constant, then the upstream temperature can be assumed to be constant also.

3) Determine the upstream density and its radial location..

4) With two values of the density on the midplane, fit an exponential curve to the points and determine the density and its gradient at the LCFS.

By fitting each timeslice, the edge density is now known. The resulting edge density from this technique can be compared to results from the reflectometer and the $\varepsilon$ analysis. In table 6.1, these values are compared. The most consistent value can be used as the best 
estimate for the background density level. To ensure the proper time history, the divertor probe signal can then be scaled and added to this background level. Although the density may not be conserved along the field lines in the scrapeoff during detachment, the time history of the density upstream should be highly correlated to the time history at the divertor and can therefore be used as a proxy for the time history of the edge density.

\subsection{Data Processing}

Before it is possible to invert for density profiles, the demodulator output must be combined and any vibrational error compensated.

\subsubsection{Phase Reconstruction}

To reconstruct the phases, any resets of 256 fringes in the fringe counters must first be removed. This is easily done by adding the appropriate \pm 250 fringes to the full phase count. The full count and partial fringe signal can then be straightforwardly added together.

The HeNe and $\mathrm{CO}_{2}$ signals are then used in eqn. 2.4 to generate the plasma phase shift. In the $100 \mathrm{~ms}$ period before the shots, the compensation should result in a zero phase shift in the absence of plasma. This period can be used to estimate the noise levels in the measurement. The four HeNe positions are first adjusted slightly to find the positions for an optimal subtraction before the shot begins. Typically, movements of approximately 1-2 mm are required. This corrects for small misalignments when lining up the $\mathrm{CO}_{2}$ and $\mathrm{HeNe}$ beams. The HeNe signals are then splined onto the positions of the $\mathrm{CO}_{2}$ channels. The $\mathrm{CO}_{2}$ positions are then also adjusted to yield the best compensations. Similarly, this procedure compensates for inaccuracies in the $\mathrm{CO}_{2}$ chord positions. 


\subsection{Experimental Checks of the Inversion}

A series of tests and checks were conducted on the inversion techniques to identify sources of systematic errors and limitations of the method. The inversion was quite robust. The phase shifts for various known density profiles were input to the analysis. Figure $4.7 \mathrm{ab}$ gives examples of the reconstruction for a peaked and a slightly hollow input profile. The inversion was able to reproduce the profiles even when randomly generated noise of up to $5 \%$ was added to the phase inputs. When the input profile became increasingly hollow, there was an increase in the error of the central density of the inverted profiles. In general, good agreement was achieved for profiles ranging from slightly hollow to peaked. Inversions of actual shots indicate that the inversion works quite well. One of the main goals of the TCI was to follow pellets. Figure 4.8 shows a good example of the inversion of a two pellet shot.

Studies done by Park indicate that inaccuracies in the magnetic flux surfaces can be a major source of error [86]. Figure 4.9 illustrates that for the SVD inversion, radial translations of the magnetic surfaces by up to $\pm 0.5 \mathrm{~cm}$ have small effects on the inverted profiles. Similarly, the inversion is relatively insensitive to those $\varepsilon$ which lie in the range of allowable values. In figure 4.10 , the inversion has been performed on the same timeslice with different values of $\varepsilon$. The range of acceptable $\varepsilon$ 's runs from 0.7 to 1.6. A minimum in the chi-square error occurs at $\varepsilon$ equal to 1.1 . From the figure, there appears to be only small differences in the inversion for the range of allowable $\varepsilon$.

In fig. 4.11 , the effects of the edge density on the inversion are shown. As the input edge density is varied by a factor of 5 , the resulting profiles goes from peaked to hollow. The edge density is the largest source of error for the inversion. Thus, it is essential that a good estimate of the edge density is made for the inversion analysis. It is worthwhile to note that the central density is less sensitive than the density at $a / 2$. The variation in the central density is $50 \%$ for a factor of 5 at the edge. Also, as the edge density increases, the first changes in the profile occur at psi values greater than 0.5 . 

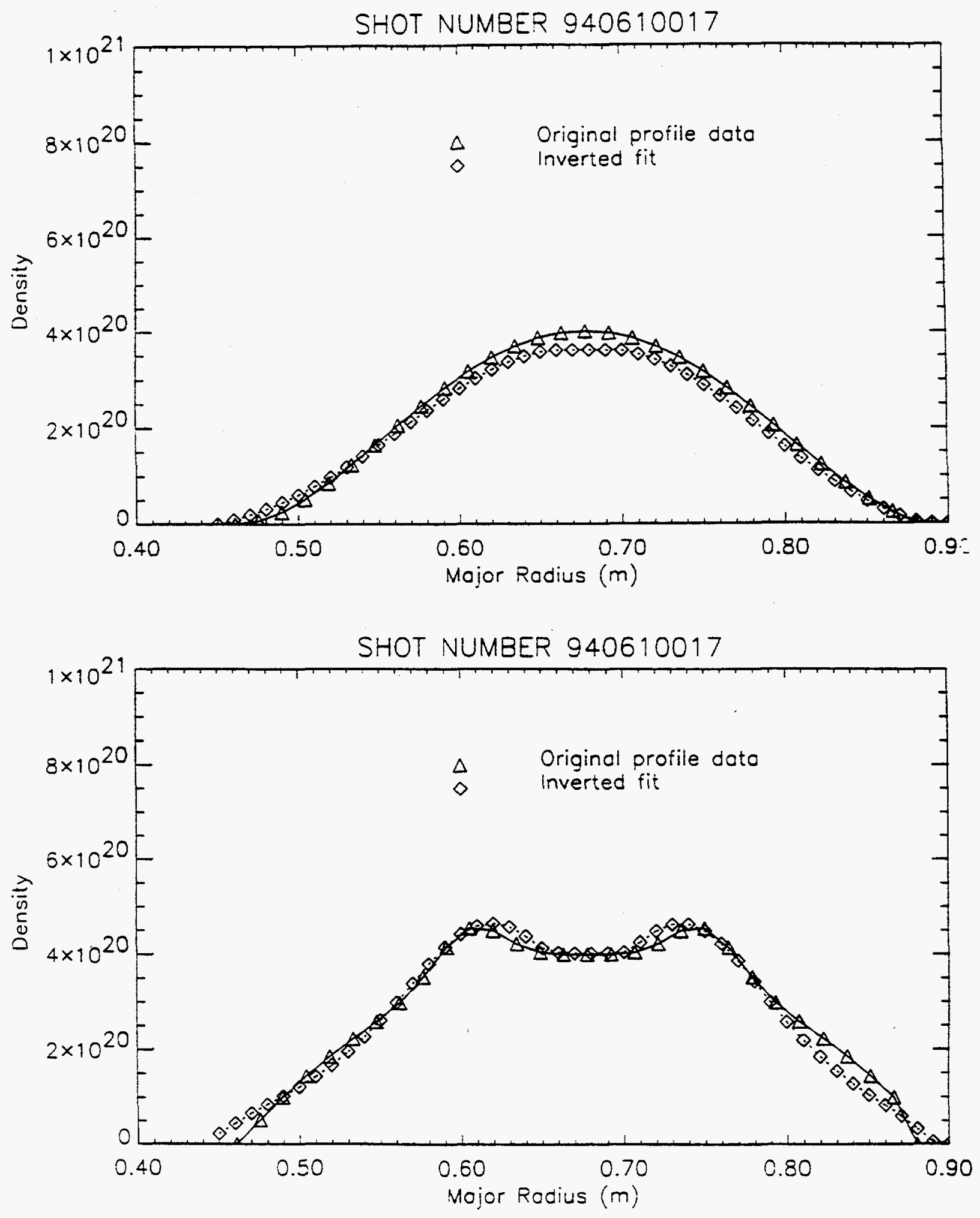

Figure 4.7: Comparison of the inverted and initial density profiles for a) a parabolic profile and b) a hollow profile. 


\section{Double Pellets: Shot 931027017}

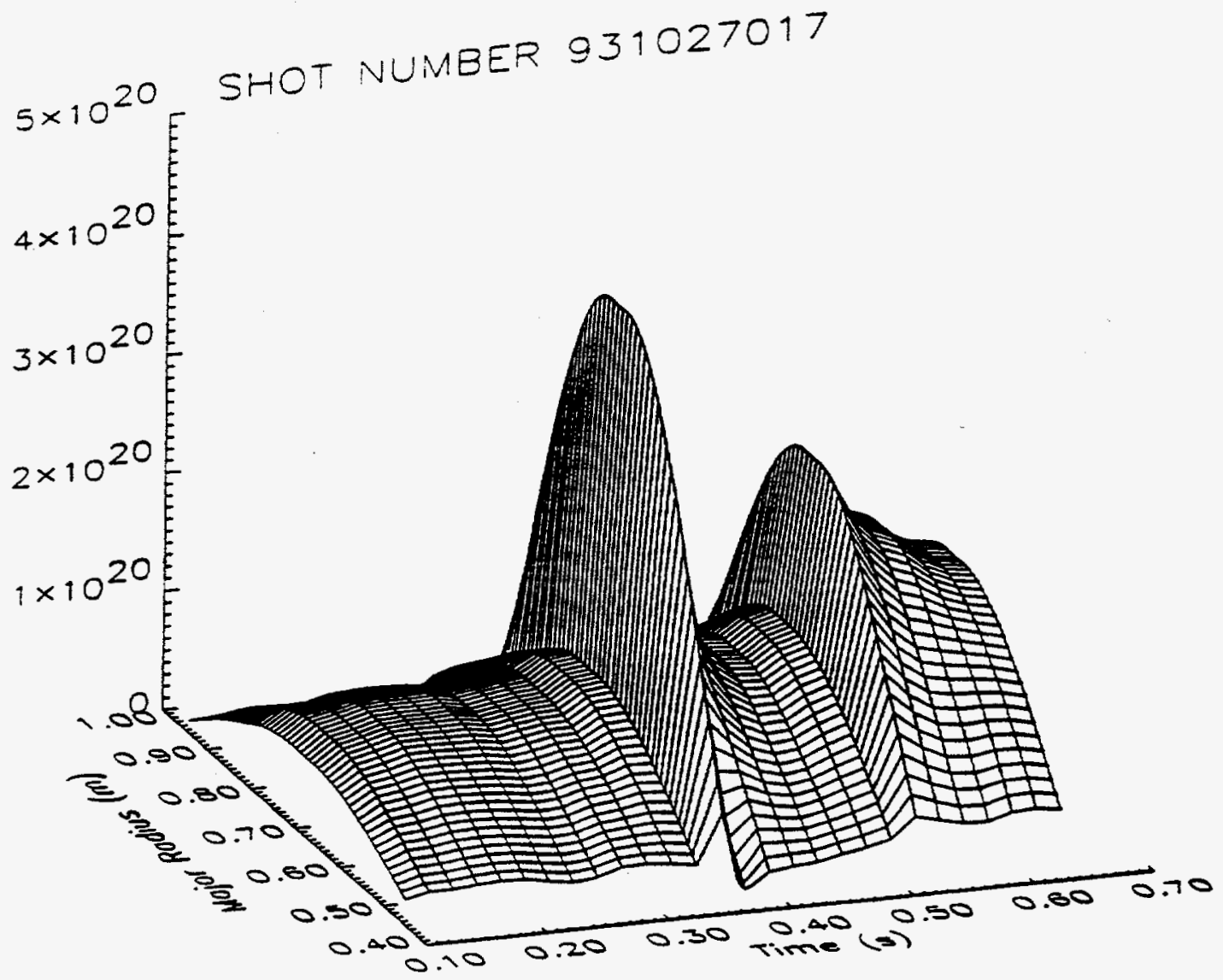

Figure 4.8: Inversion of double pellet shot. 
Thus, the presence of actual phase measurements inside of psi equal to 0.5 acts to constrain the allowable profile changes. It was also observed that the inversion using edge density values close to the best estimate (in this case .2 and $.3 \bar{n}_{e}$ ) exhibit less variation than the other curves. This implies that when the edge density is close to the true edge density, the true profile represents a convergence point for the inversion. A study on the effects of the edge gradient on the inversion also showed that the inverted profiles exhibit little change for different edge gradients. Figure 4.12 shows that the profile between $0.5<$ psi $<1.0$ adjusts to reflect the changing gradient at the LCFS but doesn't change much for psi $<0.5$. Again this reflects the fact that the phase measurements are constraining the changes in the shape of the density profile. Therefore, the magnitude of the edge density has an important role in the profile determination, while the inversion is relatively insensitive to the edge gradient, the magnetic geometry and variation of $\varepsilon$ within the allowable range. 


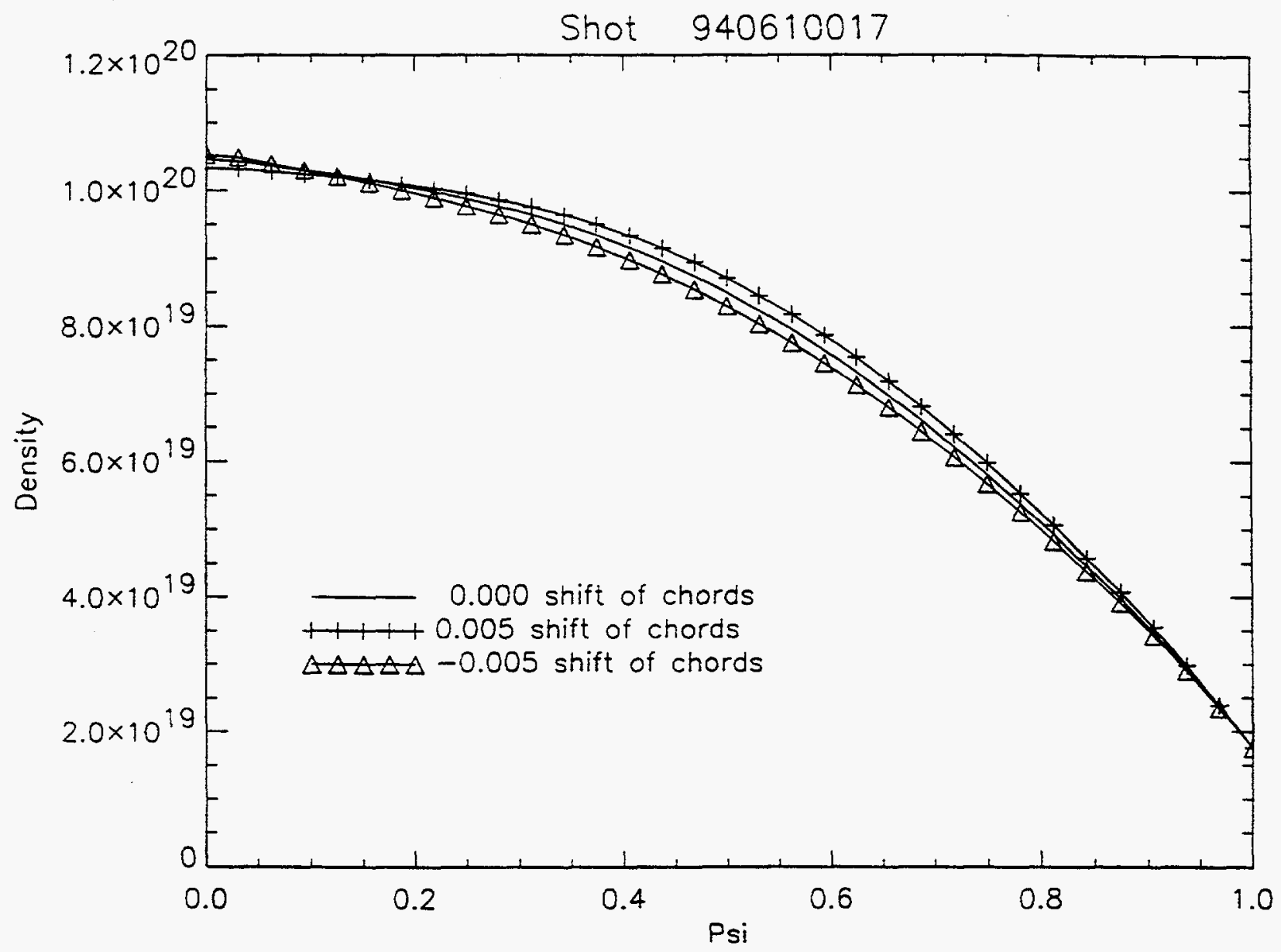

Figure 4.9: Effect of shifts in the magnetic geometry on the inverted profile. 


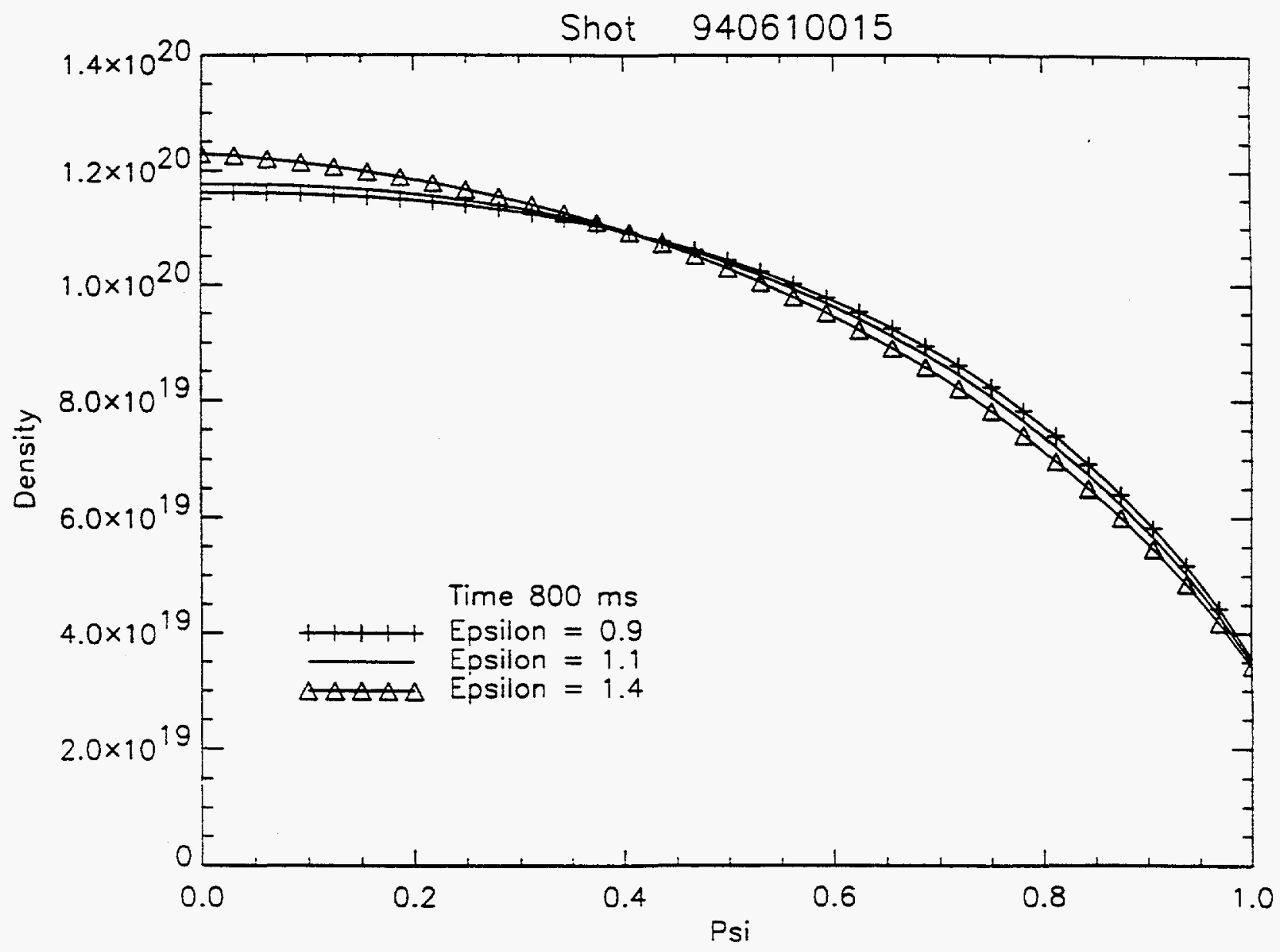

Figure 4.10: Effect of the smoothing weight, $\varepsilon$, on the inverted profile. 


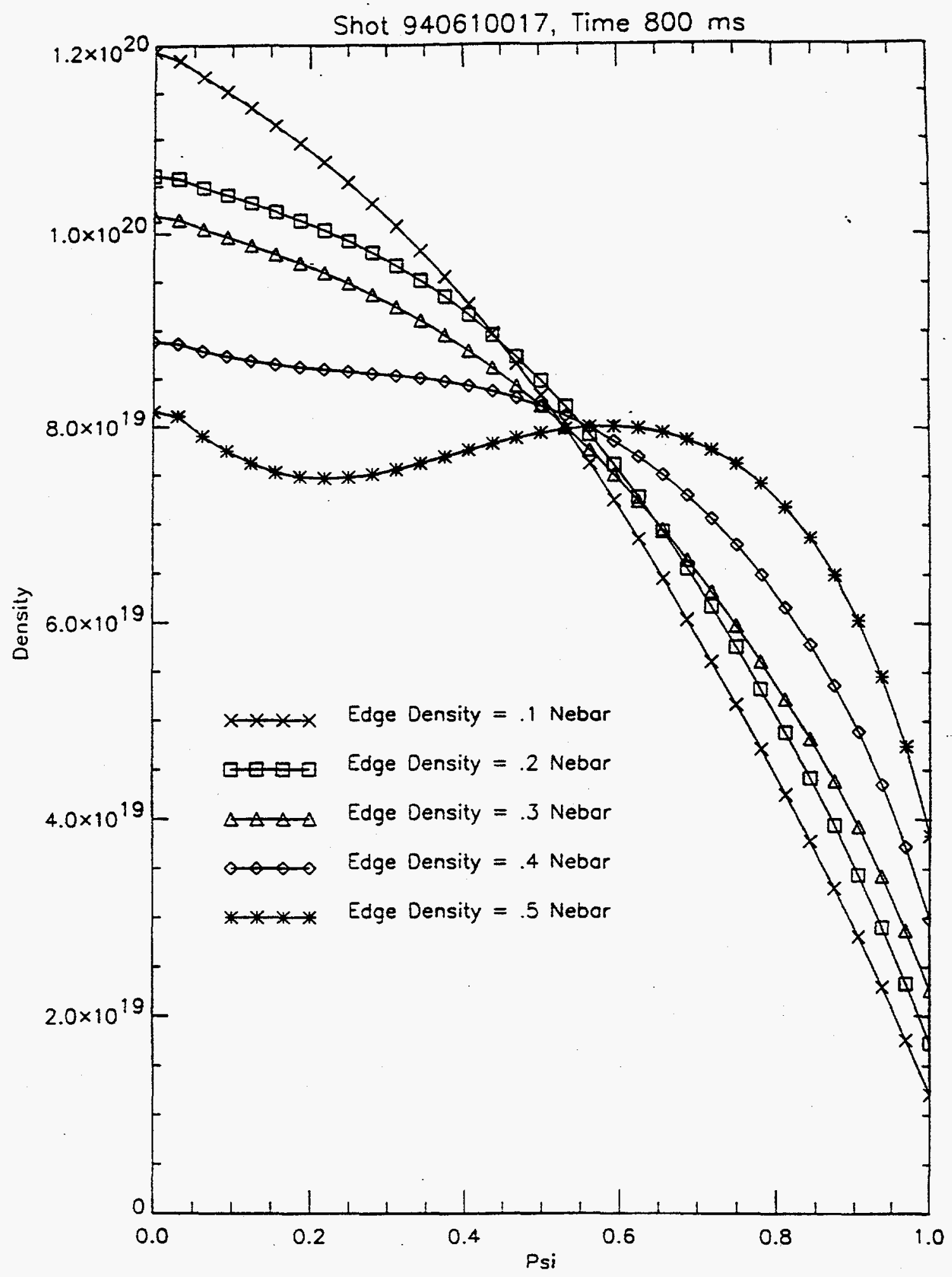

Figure 4.11: Effect of the edge density on the inverted profile. 


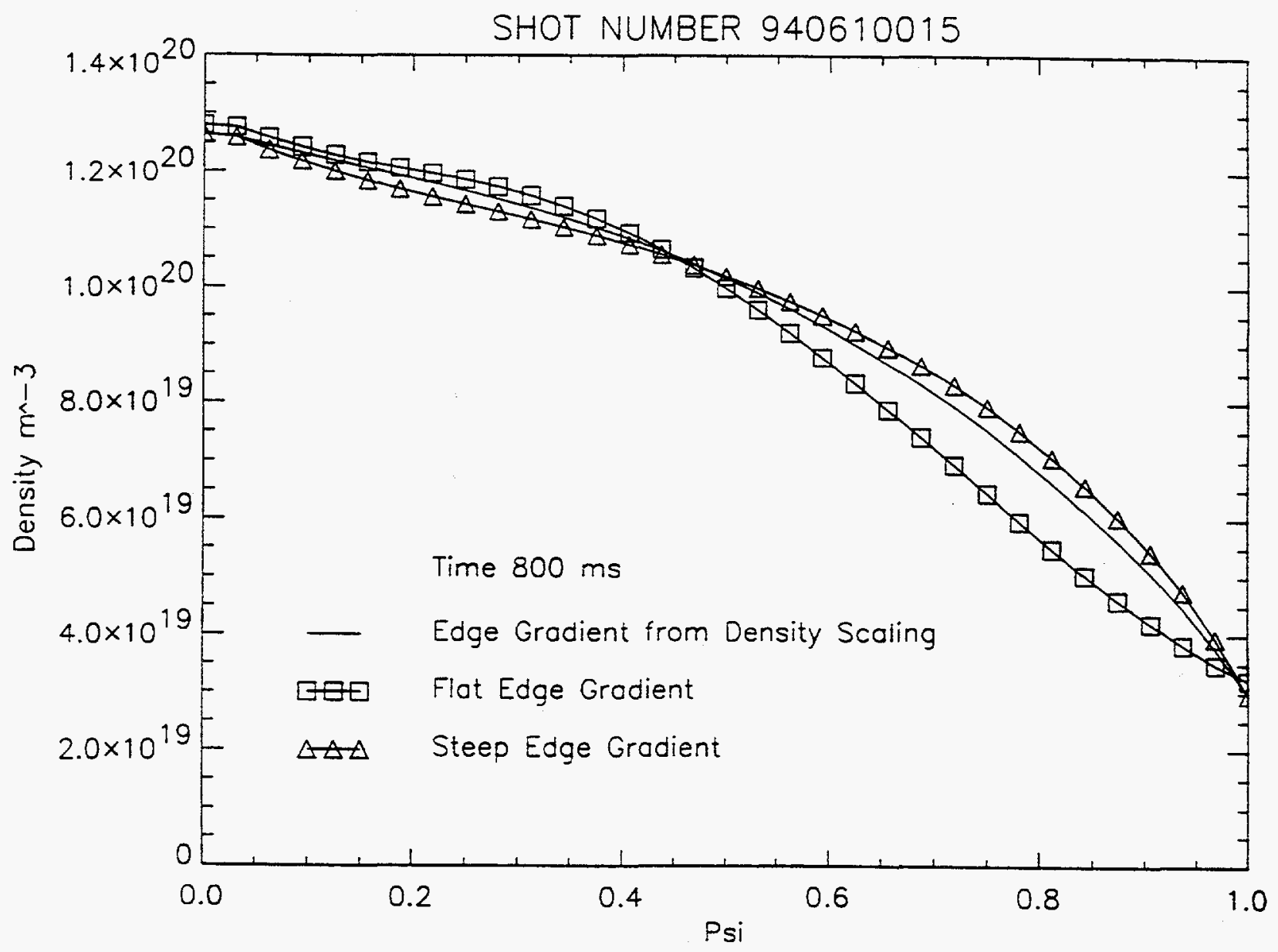

Figure 4.12: Effect of the edge gradient on the inverted profile. 


\section{Chapter 5}

\section{Transport Analysis}

To determine the transport coefficients, a two-prong analysis involving numerical simulation and regression in the time domain will be used. For each method, the exact methodology and any accompanying analysis will be presented in this chapter.

\subsection{Framework for Transport Analysis}

In fusion research, the particle and energy transport processes are driven by forces that move the plasmas towards thermal equilibrium. Gradients of density and temperature act as the sources of free energy for these fluxes. Similarly, external forces generate fluxes which drive the plasma away from equilibrium . At the point of balance, the plasma reaches a steady state or near equilibrium condition in which it can be parameterized and described by various plasma variables (e.g. $n, T_{e}, T_{j}$ ). It is in this state that the study of transport processes is possible.

\subsubsection{General Issues in Transport Analysis}

In general, transport analysis is derived from studying the velocity moments of the Boltzmann equation. By assuming that the plasma has a Maxwellian or near Maxwellian distribution function the equations governing the time evolution of plasma density, energy, and momentum can be derived [3]. Furthermore, because parallel transport is much faster than radial crossfield transport, $\mathrm{n}_{\mathrm{e}}$ and $\mathrm{T}_{\mathrm{e}}$ can be assumed to be flux quantities. Averaging over closed flux surfaces enables the equations to be reduced to a 1-D equation in $\Psi$, the flux surface parameter. The general form of a transport equation is 


$$
\frac{d g}{d t}+\frac{1}{\operatorname{Vol}^{\prime}(\Psi)} \frac{d\left(\operatorname{Vol}^{\prime}(\Psi) F\right)}{d \Psi}=S
$$

where $g$ is the state variable (e.g. $n_{e}, T_{e}, T_{i}, \ldots$ ), $F$ is the associated flux, $S$ is the source term (external or internal), $\Psi$ is the flux coordinate, and Vol is the volume enclosed by the flux surface [4]. The ' refers to a spatial derivative with respect to $\Psi$.

For particle transport, the form of the particle flux, $\mathrm{F}$ or $\Gamma$, is usually divided into two parts representing the diffusive flux and the convective flux.

$$
\Gamma=-D \frac{d n}{d \Psi}-n V
$$

$\mathrm{D}$ is the diffusion coefficient and $\mathrm{V}$ is the pinch velocity. Both coefficients are positive with a positive pinch velocity defined as outwards away from the magnetic axis. The pinch velocity is a general term that can include all convections due to temperature gradients and electric fields. This will be more obvious by comparison with eqn. 5.3.

Three equations exist for the density, momentum and temperature $\left(n_{i}, \Omega_{i}, T_{i}\right)$ for each species, $i$, but simplification through assumptions about collisionality, the mass ratios, and charge neutrality reduces that number significantly [4]. Typically, for a one ion species plasma, four equations are sufficient to describe the plasma transport: electron density, electron energy, ion energy, and angular momentum conservation.

With these equations, the basic analytical approach is to measure experimentally the time derivatives, spatial derivatives (to second order) and source terms. With this information, the fluxes can be calculated through eqn. 5.1 and then related back to the gradients of the state variables in order to deduce the transport coefficients. The resulting transport coefficients can be formulated into a transport matrix as follows, 


$$
\left[\begin{array}{c}
\Gamma \\
q_{e} \\
q_{i} \\
j
\end{array}\right]=-\left[\begin{array}{cccc}
D & n V_{E} & n V_{i} & n V_{N C} \\
q_{e c o n v} & \chi_{e} & q_{p e i} & q_{e E} \\
q_{i c o n v} & q_{p i e} & \chi_{i} & q_{i E} \\
j_{n} & j_{e} & j_{i} & \sigma
\end{array}\right]\left[\begin{array}{c}
\frac{\partial n}{\partial \rho} \\
\frac{\partial T_{e}}{\partial \rho} \\
\frac{\partial T_{i}}{\partial \rho} \\
E
\end{array}\right] .
$$

This matrix is symmetric for classical and neo-classical transport. However for turbulent transport, this symmetry may not hold. Also, if the coefficients are non-linear (have dependencies on the state variables and their derivatives) this analysis is not sufficient.

\subsection{Particle Balance Analysis}

A standard technique in transport analysis is to investigate the plasma in equilibrium. In this case, the time derivative is zero and the divergence of the flux balances the source terms within a given flux surface. This method allows for all coefficients in any row of the matrix to be determined although only diagonal terms are typically considered significant.

The advantages of such a global analysis are its generality and ease of use. The disadvantages are the need for well-measured density and temperature profiles and accurate calculation of the source terms. The source term can be a quantity quite difficult to measure. For energy measurements, in order to get the exact ion-electron coupling, accurate measurement of both $\mathrm{T}_{\mathrm{e}}$ and $\mathrm{T}_{\mathrm{i}}$ must be available. Also, there is a limitation to the number of coefficients that can be measured. With only four equations for a standard one ion plasma, only four coefficients out of ten independent quantities can be determined. Though many of these coefficients are insignificant, there is growing evidence that some 
of the off-diagonal terms that represent coupling between fluxes are non-negligible [ 91 , 92, 93, 94]. This analysis will not be able to account for such mechanisms. Finally, for spatial regions in which the source term is insignificant, only the ratio of the coefficients

can be found, For particle transport, this means that only $-\frac{V}{D}=\frac{1}{n} \frac{\partial n}{\partial r}=$ inverse length scale of profile can be uniquely determined. To resolve each individual coefficient, either a temporal variation or spatial source profile must be incorporated into the analysis.

\subsection{Perturbation Analysis}

Another widely used technique to analyze plasma transport is through perturbation experiments. In such an analysis, small perturbations of the state variable are created on top of a steady state background. The time evolution of these perturbations is then examined by linearizing the transport equations and calculating the fluxes. The relationship between the gradients of the state variables and the flux determine the diffusion coefficients. This methodology offers 1) improved accuracy since signals can be time averaged over several perturbations cycles, 2) the ability to measure local coefficients when the source itself is localized, 3) a well-defined source term, and 4) the opportunity to measure all the elements of the transport matrix. However, this type of analysis requires a diagnostic set with better temporal and spatial resolution. Furthermore, it is possible for a large perturbation (e.g., pellet injection) to invalidate the analysis since the transport may be non-linear.

Perturbation analysis is complementary to particle balance analysis. As pointed out by Cardozo, an equilibrium analysis gives the ratio of the flux to the gradient, for example $\mathrm{D}=\Gamma / \nabla \mathrm{n}[95]$. Perturbations allow the measurement of incremental coefficients or the instantaneous increase of flux due to changes in the gradients, $D_{\text {inc }}=\partial \Gamma / \partial \nabla n$. The difference is depicted pictorially in fig. 5.1. 


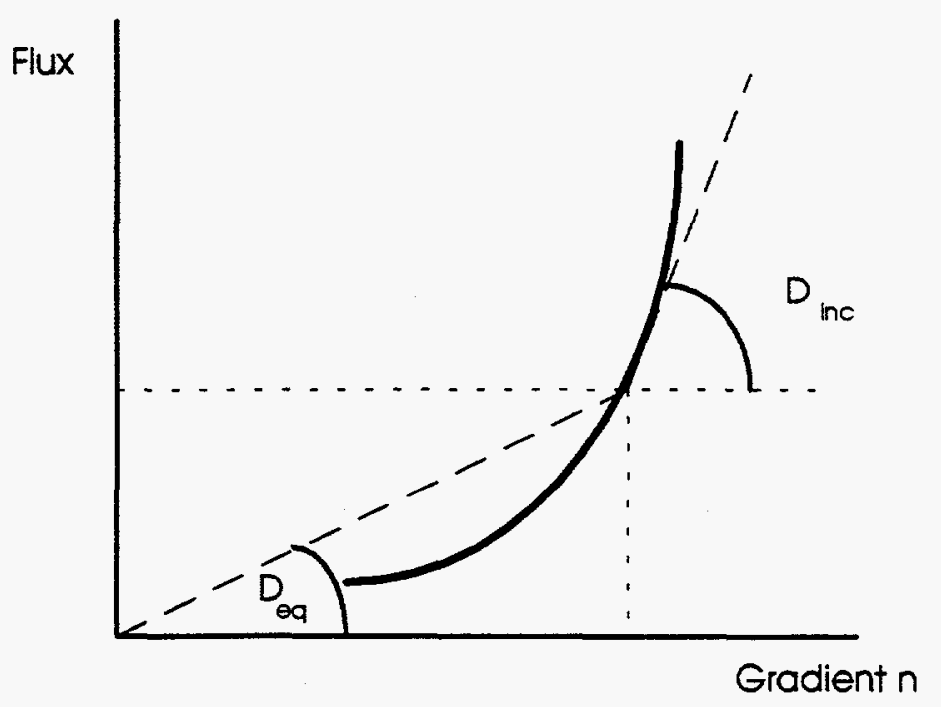

Figure 5.1: Pictorial representation of $D_{\text {eq }}$ and $D_{\text {inc. }}$ [95]

\subsubsection{Previous Results of Perturbation Analysis}

The use of perturbative analysis has been increasing with the development of temperature and density diagnostics with greater resolution and sensitivity. The study of sawteeth, pioneered by Callen and Jahns, has been performed at many different machines including Alcator C $[96,100]$. Measurements of the heat pulse are done typically with an ECE polychrometer with the corresponding density perturbation measured by either an interferometer or reflectometer $[97,98]$. On JET, both the outgoing heat pulse and the inward density pulse were observed. Diffusion coefficients were measured to be $D(r)=$ $(0.16 \pm 0.02)\left(1+\alpha \mathrm{r}^{2} / \mathrm{a}^{2}\right) \mathrm{m}^{2} / \mathrm{s}$ with a correspondingly high energy diffusivity, $\chi_{\mathrm{e}}$, of $3-5$ $\mathrm{m}^{2} / \mathrm{s}$. These values were consistent with the high $\chi_{\mathrm{e}} \mathrm{D}$ ratios measured by equilibrium studies [99].

Another frequently used perturbation is the injection of hydrogen or deuterium pellets into the plasma $[101,102,103]$. Diffusion coefficients were determined by simulating the decay of these large amplitude perturbations. By using the form of the flux as given by eqn. 5.2, good agreement between simulated and measured results was 
obtained. However, the deduced values of the diffusion coefficients were much larger than the neoclassical predictions. In all cases, a nonzero pinch velocity was needed to explain the measured data.

A smaller perturbation to the plasma can be induced by gas puffing. Modulated gas puffing experiments were first performed on T-10 by Bagdasarov [104]. Similar applications of gas puffing have been repeated on TEXT [105], ASDEX [106], JT-60 [107], MTX [74]and TFTR [108, 109]. Such experiments are popular because it allows for a controlled and repeatable perturbation. A variety of analyses, ranging from a Fourier analysis of the fundamental component of the perturbation to fitting with simulations can be performed. With better diagnostics, local measurements of the diffusion coefficients can be measured as opposed to simple fits of functional forms $[74,108]$.

Results confirm that a substantial inward pinch is required to match observed data. There also seems to be a consensus on the inverse scaling of $\mathrm{D}$ with density. This result was seen by Efthimion at TFTR, Gentile at ASDEX and also by Gentile at TEXT [105, 106, 108]. At TEXT an additional but weak dependence on $q$ was noted. This inverse scaling is consistent with the observation that particle confinement is initially increasing with density and then saturates. As observed in pellet injection experiments, the size of the perturbation can influence the transport. It was observed that there was little variation in the measured coefficients for perturbations in which the $\Delta \mathrm{n} / \mathrm{n}$ was less than $10 \%$ [108].

Another consistent observation is the increasing value of $\mathrm{D}$ and $\mathrm{V}$ towards the edge. This was seen clearly by Marinak at MTX and Efthimion at TFTR [74. 108]. Results were similar at TEXT but are less conclusive since this feature was built into the model for D. On ASDEX, Gentile found that the data could be fit by a constant value except when the density was near the density limit. In those cases, radially increasing diffusion coefficients were necessary. It should be noted that the ASDEX result does not preclude a spatially varying diffusion coefficient because such an analysis was not performed [106]. 


\subsection{Effects of Non-linearity of the Diffusion Coefficients}

It would be impossible to discuss all the separate issues involved in particle, energy and momentum transport in this thesis. Thus, in the remainder of this chapter, discussions of the transport analysis will be restricted to particle transport alone.

In the continuity equation, 5.1, one of the biggest assumptions required is the form of the $\mathrm{D}$ and $\mathrm{V}$ coefficients. Typically, the coefficients are defined as constant or as a polynomial function of $\mathrm{r}$ ( e.g. $\mathrm{V}=\frac{r}{a}$ or $\mathrm{V}=\frac{r^{2}}{a^{2}}$ ). A more complicated formalism has been developed by Gentile [94] that allows for forms of $D, V$ that depend nonlinearly on $\mathrm{T}_{\mathrm{e}}$ and $\mathrm{n}_{\mathrm{e}}$ and their spatial derivatives. The consequences of such dependencies are: 1) a divergence from the equilibrium diffusion coefficients when describing fast perturbations and 2) a coupling between $T_{e}$ and $n_{e}$ when dependencies on the $n_{e}$ and $T_{e}$ gradients exist. In the analysis of Gentile, which assumes a slab geometry, linearization of eqn 5.1 in $n_{e}$, $\mathrm{T}_{\mathrm{e}}, \nabla \mathrm{n}$, and $\nabla \mathrm{T}$ about the equilibrium solution, leads to the following expansions for the flux and the continuity equation

$$
\begin{gathered}
-\tilde{\Gamma}=D \frac{d \tilde{n}}{d x}+V \tilde{n}+\left(\tilde{n}_{x} \frac{d D}{d \tilde{n}_{x}}+\tilde{n} \frac{d D}{d n}+\tilde{T}_{x} \frac{d D}{d T_{x}}+\tilde{T} \frac{d D}{d T}\right) \frac{d n_{0}}{d x}+ \\
\left(\tilde{n}_{x} \frac{d V}{d \tilde{n}_{x}}+\tilde{n} \frac{d V}{d n}+\tilde{T}_{x} \frac{d V}{d T_{x}}+\tilde{T} \frac{d V}{d T}\right) n_{0}
\end{gathered},
$$

where $n_{x}$ and $T_{x}$ represent $\nabla \mathrm{n}$ and $\nabla \mathrm{T}$. 


$$
\begin{aligned}
\frac{d \tilde{n}}{d t} & =\left(D+\frac{\partial D}{\partial n_{x}} \frac{\partial n_{0}}{\partial x}+\frac{\partial V}{\partial n_{x}} n_{0}\right) \frac{\partial^{2} \tilde{n}}{\partial x^{2}}+\left(\frac{\partial D}{\partial T_{x}} \frac{\partial n_{0}}{\partial x}+\frac{\partial V}{\partial T_{x}} n_{0}\right) \frac{\partial^{2} \tilde{T}}{\partial x^{2}} \\
& +\left[\frac{d D}{d x}+V+\frac{d}{d x}\left(\frac{\partial D}{\partial n_{x}} \frac{\partial n_{0}}{\partial x}\right)+\frac{\partial D}{\partial n} \frac{\partial n_{0}}{\partial x}+\frac{\partial V}{\partial n} n_{0}+\frac{d}{d x}\left(\frac{\partial V}{\partial n_{x}} n_{0}\right)\right] \frac{d \tilde{n}}{d x} \\
& +\left[\frac{d V}{d x}+\frac{d}{d x}\left(\frac{\partial D}{\partial n} \frac{\partial n_{0}}{\partial x}\right)+\frac{d}{d x}\left(\frac{\partial V}{\partial n} n_{0}\right)\right] \tilde{n} \\
& +\left[\frac{d}{d x}\left(\frac{\partial D}{\partial T_{x}} \frac{\partial n_{0}}{\partial x}\right)+\frac{\partial D}{\partial T} \frac{\partial n_{0}}{\partial x}+\frac{d}{d x}\left(\frac{\partial V}{\partial T_{x}} n_{0}\right)+\frac{\partial V}{\partial T} n_{0}\right] \frac{d \tilde{T}}{d x} \\
& +\left[\frac{d}{d x}\left(\frac{\partial D}{\partial T} \frac{\partial n_{0}}{\partial x}\right)+\frac{d}{d x}\left(\frac{\partial V}{\partial T} n_{0}\right)\right] \tilde{T}
\end{aligned}
$$

with

$$
\frac{d}{d x}=\left(\frac{\partial^{2} n_{0}}{\partial x^{2}}\right) \frac{\partial}{\partial n_{x}}+\left(\frac{\partial n_{0}}{\partial x}\right) \frac{\partial}{\partial n}+\left(\frac{\partial^{2} T_{0}}{\partial x^{2}}\right) \frac{\partial}{\partial T_{x}}+\left(\frac{\partial T_{0}}{\partial x}\right) \frac{\partial}{\partial T}+\frac{\partial}{\partial x}
$$

It is clear that when there are dependencies of $\mathrm{D}$ and $\mathrm{V}$ on $\nabla \mathrm{n}$ and $\nabla \mathrm{T}$ the effective diffusion coefficients for the perturbation are changed.

While it is difficult to experimentally separate the individual dependencies, various simplifying assumptions can lead to qualitative insights about the resulting transport. The first is that if the perturbation evolves on a timescale faster than the equilibration time, then convective effects are small and only the highest order time derivatives need to be retained in the solution. Also, gradients in $\mathrm{T}$ and $\mathrm{n}$ act as sources of free energy to drive the anomalous transport. A simple dependence of the coefficients on $\nabla \mathrm{n}$ results in enhanced diffusion. Similarly, any spatial dependence (direct or indirect) of the coefficients results in extra convection. Gentile showed further that a dependence on $\nabla T$ results in a coupling of the energy and particle transport equations [94]. 


$$
\frac{\partial \tilde{n}}{\partial t}=\left(D+\frac{\partial D}{\partial n_{x}} \frac{\partial n_{0}}{\partial x}+\frac{\partial V}{\partial n_{x}} n_{0}\right) \frac{\partial^{2} \tilde{n}}{\partial x^{2}}+\left(\frac{\partial D}{\partial T_{x}} \frac{\partial n_{0}}{\partial x}+\frac{\partial V}{\partial T_{x}} n_{0}\right) \frac{\partial^{2} \tilde{T}}{\partial x^{2}}
$$

$$
\begin{aligned}
\frac{3}{2} \frac{\partial \tilde{T}}{\partial t} & =\left(\frac{T_{0}}{n_{0}}\right) \frac{\partial \tilde{n}}{\partial t}+\left(\frac{\partial \chi}{\partial n_{x}} \frac{\partial T_{0}}{\partial x}+1.5 T_{0} \frac{\partial U}{\partial n_{x}}\right) \frac{\partial^{2} \tilde{n}}{\partial x^{2}} \\
& +\left(\chi+\frac{\partial \chi}{\partial T_{x}} \frac{\partial T_{0}}{\partial x}+1.5 T_{0} \frac{\partial U}{\partial T_{x}}\right) \frac{\partial^{2} \tilde{T}}{\partial x^{2}}
\end{aligned}
$$

These equations can be solved as a coupled oscillator problem with fast and slow solutions. The fast solution corresponds to a diffusion rate higher than the equilibrium rate and the slow solution reflects transport at a rate slightly less than equilibrium.

Although $\mathrm{D}$ and $\mathrm{V}$ are defined as functions of space, in reality, the actual profiles are function not of position but of the plasma parameters at that location. The analysis of Gentile indicates that equilibrium transport coefficients will be modified if dependencies on the state variables exist. However, it is virtually impossible to extract these dependencies experimentally. The usefulness of such analysis is in the fact that experimental profiles can place constraints on the allowable dependencies. For example, experimental observations indicate the need for a strong inward pinch [101]. Any model that depends only on $\nabla \mathrm{n}$ and $\nabla \mathrm{T}$ would not exhibit this required behavior and could therefore be disproved. This methodology provides a useful and testable tool to link theoretical models and experimental evidence. 


\subsection{Regression Analysis}

In order to determine the diffusion coefficients, a multilinear regression technique was developed and implemented. Developed in 1886 by Francis Galton to study the height of children relative to their parents, regression analysis has become a versatile and invaluable technique to study statistically the dependence of one variable on one or more independent quantities [110].

Typically, for perturbative experiments, it is often the practice to linearize the transport equation and work with perturbed quantities by subtracting off an appropriate equilibrium profile. In the regression analysis, this is unnecessary and can actually introduce additional error if the equilibrium profile is incorrect. Starting with eqn 5.1, the continuity equation, the first step is to take a volume integral over a flux surface $\Psi$.

$$
\oint_{V(\Psi)} d V \frac{d n}{d t}=\oint_{V(\Psi)}(-\nabla \cdot \Gamma+S(\rho)) d V
$$

Here, the particle flux, $\Gamma$, is assumed to have the form given by eqn. 5.2. For surfaces deep in the plasma core (e.g. more than $5 \mathrm{~cm}$ inside the LCFS), the source term can be neglected. Thus, the right hand side (RHS) is reduced to the single integral over the divergence of the flux which can be converted to a surface integral by Gauss' Law. On the left hand side (LHS), the order of the time derivative and the volume integral are interchanged. This change gives

$$
\frac{d \oint d V n}{d t}=\oint_{s(\Psi)}\left(D \frac{d n}{d \Psi}+n V\right) d a
$$

Defining $\mathrm{N}_{\text {part }}$ to be the volume integral over $\mathrm{n}$ and assuming that the flux is constant over the surface, it is clear that for each flux surface there is a linear relationship between 
the time derivative of the particles inside the surface and the density and first spatial derivative on the surface. Defining $A(\Psi)$ as the surface area of the flux surface,

$$
\frac{d N_{\text {part }}}{d t}=D\left(\frac{d n}{d \Psi} A(\Psi)\right)+V(n A(\Psi))
$$

From the density profiles, all three quantities can be calculated as a function of time. The surface area and volume of the flux surfaces are provided by EFIT. By performing the multilinear regression over many timeslices, the coefficients of the regression or diffusion coefficients can be extracted as functions of both time and space.

\subsection{Numerical Simulation}

A second method of extracting the diffusion coefficients is through numerical simulation of the time history of the interferometer signals [111]. Since the equation of motion is known for the particle density, eqn 5.1, it is possible to simulate the behavior of the density profiles under gas modulation. This problem can be set up in a very similar fashion to the regression analysis. However, in this case, the analysis is predictive. By assuming some profile for $\mathrm{D}$ and $\mathrm{V}$, the time history of the density profiles can be simulated and the predicted measurements for the TCI can be computed by knowing the magnetic geometry. These predicted signals can then be compared to the actual measurements and the diffusion coefficients can be adjusted until the time behavior fits the observations. A unique model of the coefficient profiles allows the central and edge values of the profile to be adjusted independently. The general form for the transport coefficient $\mathrm{C}(\mathrm{r})$ is given by

$$
C(r)=\frac{C_{1}}{\left(1+C_{0}\right)}\left(r^{2}+C_{0} r^{8}\right),
$$


where $r$ is a normalized radius. Figure 5.2 shows that for different values of the parameter $\mathrm{C}_{0}$, the central coefficient profile flattens or steepens without affecting the edge. The edge value can then be matched to any desired value by scaling the entire profile.

In addition, the source size at the edge is set so that throughout the simulation the total particle inventory is matched. This includes a period of $10 \mathrm{~ms}$ before the puff, when the plasma should be near equilibrium. This extra condition is enough to uniquely determine the problem. For a given equilibrium profile, the ratio V/D is uniquely determined. For this V/D ratio, only one source size will accurately match the density on axis. The exact details of this source is inconsequential in determining the magnitude of the density profile in the core because the source is highly localized at the edge [106]. All that is necessary is the total source size. In the simulation for Alcator C-Mod, a Gaussian profile is used. It is centered on the LCFS with a width of $1 \mathrm{~cm}$. Details of the source term and tests of the sensitivity of the simulation to the source profile are given in Appendix A. However, there is still a family of curves that satisfy the same V/D ratio. By fitting the time history of the interferometer chords, this last free parameter is determined.

This analysis was implemented and used for the gas puffing experiments. Results are presented in the section 6.3 and details of the simulation are presented in Appendix A.

\subsection{Tests of the Analysis Techniques}

In order to ensure the validity of the output from both the regression and simulation analysis, tests were conducted on both methods. The diffusion equation can be solved in closed form for several simple cases. The simplest is that of a constant diffusion coefficient and a pinch velocity of zero in a circular geometry. The analytic solution for this case is given by

$$
n(r, t)=n_{0} e^{-\left(\frac{D(2.4)^{2} t}{a^{2}}\right)} J_{0}\left(\frac{2.4 \sqrt{D}}{a} r\right)
$$




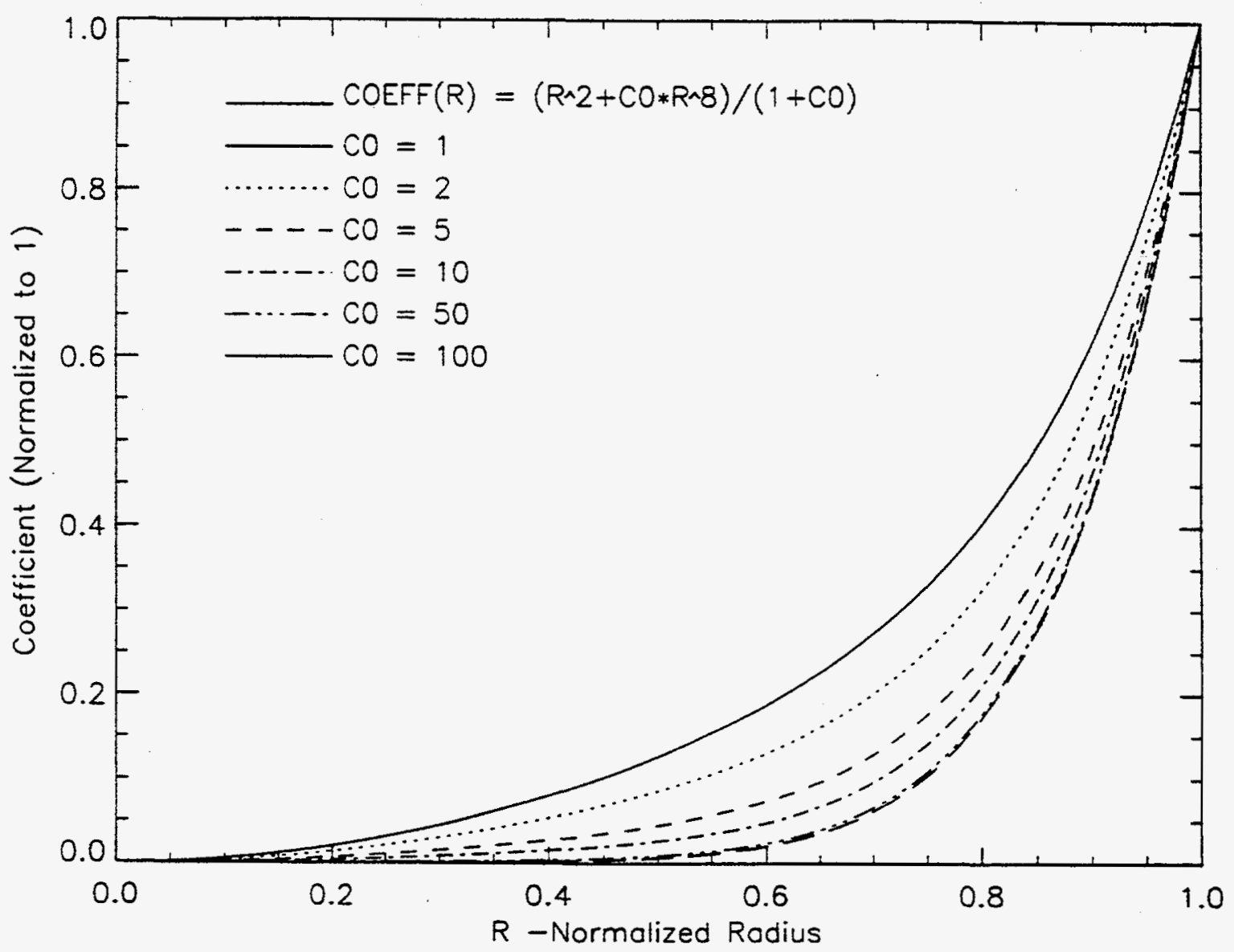

Figure 5.2: Comparison of coefficient profile as a function of $\mathrm{C} 0$. 
where $\mathrm{a}$ is the minor radius, $\mathrm{D}$ is the diffusion coefficient, and $\mathrm{n}_{0}$ is the initial central density. Tests of the simulation with the above conditions gave exact agreement between the simulated profiles and the analytic solution.

To test the regression technique, a $100 \mathrm{~ms}$ period was generated with the simulation. The simulation included a gas puff from the edge at $25 \mathrm{~ms}$ for $25 \mathrm{~ms}$. The resulting time history of the density profiles was input into the regression analysis and the resulting diffusion coefficients were compared with the original coefficient profiles used in the simulation. Figure 5.3 illustrates the very good agreement between the input and regressed coefficient profiles even when $1 \%$ or roughly $3^{\circ}$ of noise was added to the profiles. The results of these two tests gives additional validation to our analysis technique. Sensitivities of the resulting coefficient profiles to the assumptions made in the analysis will be presented in the next chapter. 
Shot 940610015
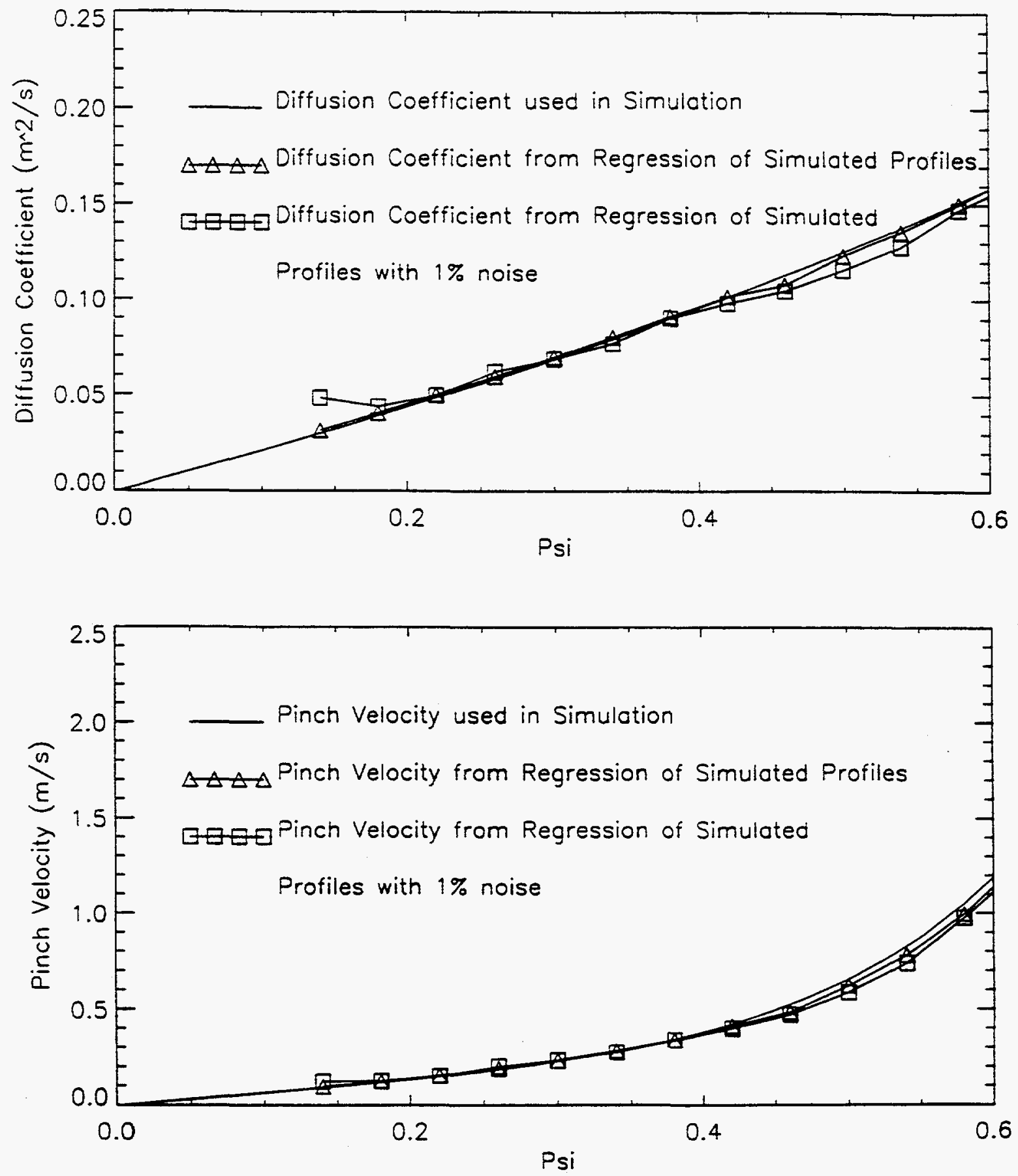

Figure 5.3: Comparison of simulated coefficient profile with regressed profiles using $0 \%$ and $1 \%$ noise. 


\section{Chapter 6}

\section{Experimental Observations}

\subsection{Overview}

There are many issues involved with a proper inversion and transport analysis. In this chapter, the results of both the inversion and transport analysis for the gas modulated shots will be discussed. The chapter will be divided as follows. Section 6.2 will examine the phenomenology of the gas puffing and the resulting density profiles. Especially important are the various sensitivities of these profiles to sources of systematic errors such as the determination of an appropriate edge density and the proper choice of weighting for the smoothing. The next section will review the results of the regression analysis used to extract the diffusion coefficients. Again, attention will be paid to justifying the various assumptions made in order to perform the regressions. Section 6.4 will present the results of the simulation analysis. Agreement between the simulation and regression analysis was good.

\subsection{Phenomenology of Gas Puffing}

A series of gas modulation experiments was performed for a range of line integrated densities at the same toroidal field and plasma current. The plasma was diverted into the lower divertor at approximately $600 \mathrm{~ms}$. The plasma was either immediately detached or attached depending on whether the line integrated density at the time of diversion was above or below the detachment threshold. The line averaged electron density was varied between $7.0 \times 10^{19}-1.4 \times 10^{20} \mathrm{~m}^{-3}$. Three or four gas puffs were injected from a piezoelectric valve on horizontal Port $A$ at a frequency of $10 \mathrm{~Hz}$. To generate the puffs, the voltage to the valve was increased by $30-40 \mathrm{~V}$ for $10-20 \mathrm{~ms}$. 
The puffs were clearly visible on the line integrated densities. Figure 6.1 shows the time history of several of the interferometer chords before and after a single gas puff. The density begins to rise a few milliseconds after the puff is injected and peaks roughly $5 \mathrm{~ms}$ after the puff stops. The density perturbation slowly decays over the next $60 \mathrm{~ms}$ back to their original level. The size of the puffs was $3-5 \times 10^{19} \mathrm{~m}^{-3}$ particles and represents between $5-10 \%$ of the total particle inventory. In terms of the peak density, the fractional change was typically $7 \%$. The profile generally broadened with the puffs and peaked up as the puffs decayed away. This effect can be seen in the slight modulation of the peaking factor, peak density over volume averaged density, as the puffs were injected.

The first puff was injected at 650 or $700 \mathrm{~ms}$ which allowed $50-100 \mathrm{~ms}$ for the plasma to reach equilibrium after diverting. It appears, however, that in many of the shots, the plasma was not fully relaxed by the time of the first puff. The density was still rising and the position of the x-point was still moving. The first puff would have an extraordinarily large rise or the density didn't decay afterwards. By the second and third puffs, the plasma had stabilized and the density rose and fell back to the same level. In fig. 6.2 , the first puff at $700 \mathrm{~ms}$ didn't decay at all and continued to rise until the puff at 800 ms. The second and third puffs behaved as expected.

\subsubsection{Stability of the Shots}

It is essential in a perturbation experiment to isolate the source of the perturbations. As a result, the shots must be as stable and as consistent as possible. The position and the shape of the plasma should not be changing during gas puffing. Otherwise, the changes in density profile due to transport will be confused with those due to shifts in the magnetic configuration. Measurements by the magnetic diagnostics and the EFIT reconstructions of the flux surfaces indicated that there was very little change in the plasma position and shape. The shots were all taken at the same plasma current of $630 \mathrm{kA}$ and toroidal field of 5.2. T. The plasma major radius was typically $.678 \mathrm{~m}$ with 


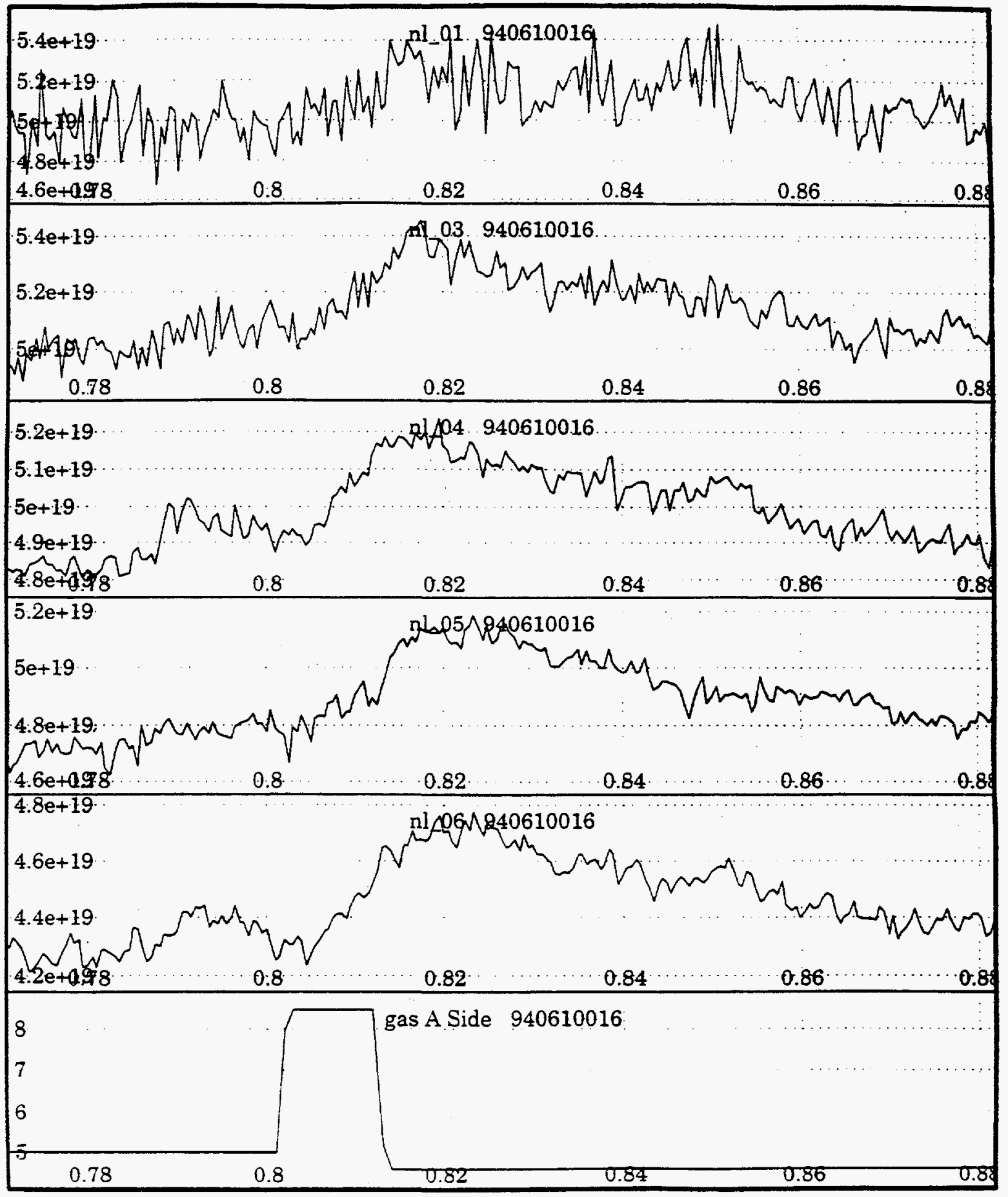

Figure 6.1: Interferometer signals on chords $1,3,4,5$, and 6 during gas puff. 


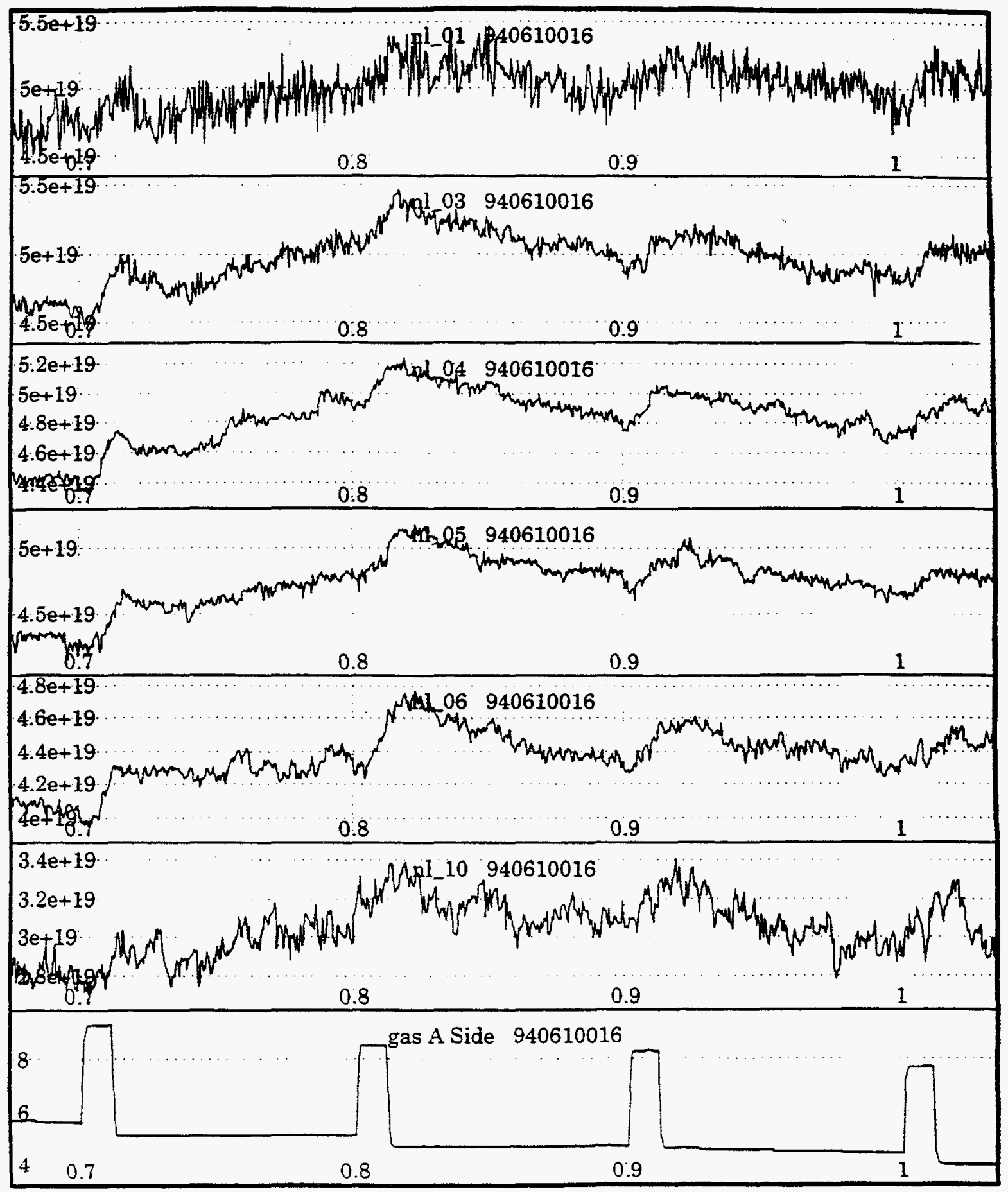

Figure 6.2: Interferometer signals for repeated gas puffs. 
movements of less than $2 \mathrm{~mm}$ over the time of the puffs. The minor radius varied at most $1 \mathrm{~cm}$ from its nominal value of $21 \mathrm{~cm}$. Plasma elongation varied between 1.6 and 1.8 but for each shot the variation was less than $2 \%$. Figure 6.3 shows the typical range of variation for the position of the magnetic axis, Kappa and minor radius. The bottom trace indicates the timing and duration of gas puffs from the piezoelectric valve.

\subsubsection{Profile Inversions}

The inversion analysis was performed on the gas modulated shots of $94 / 06 / 10$. The standard processed outputs of the inversion, as shown in figure 6.4 are: the total particle inventory, line averaged density, the peak density and the peaking factor. The puffs can be seen in each of these signals. Of special note is the dips in the peaking factor as the puffs are injected. The profile broadened as the gas was injected and raised the edge density before being transported into the main plasma. In fig 6.5 the time evolution of the inversion shows the effects of the puff. Profiles rose and broadened $(820 \mathrm{~ms})$ and then peaked up as the density on axis continued rising but the edge fell $(860 \mathrm{~ms})$. After $860 \mathrm{~ms}$, the profile returned to its initial shape and the next puff was injected at $900 \mathrm{~ms}$. The inverted profiles in general were peaked, with hollow profiles being rare, even during puffing. The typical peaking factors during the gas puffing period ranged from 1.5 to 2.0 with a trend towards higher peaking for the lower density shots.

\subsubsection{Results of Analysis to Determine Smoothing Weight}

In chapter 4 , a technique was prescribed to determine the proper smoothing factor to be used in the inversion. For edge densities ranging from 0.1 to $0.5 \bar{n}_{e}$, this procedure was applied. In figure 6.6 , both the error in fitting the edge and the error in the chi-

squared distribution is shown for a range of edge densities from $0.7-1.3 \times 10^{20} \mathrm{~m}^{-3}$. 


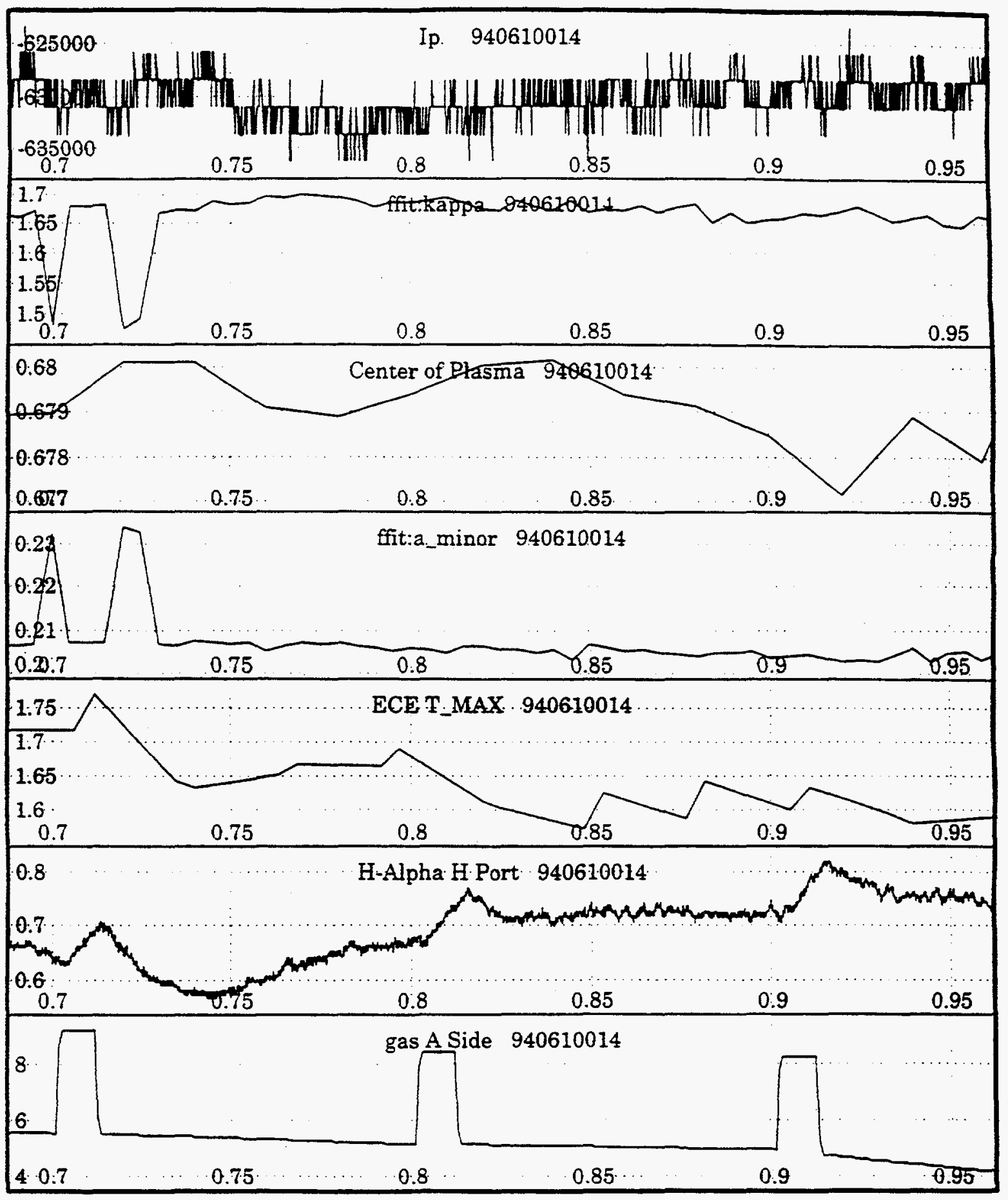

Figure 6.3: Time histories of plasma parameters during gas modulation. All parameters exhibit good stability during the puffing. 


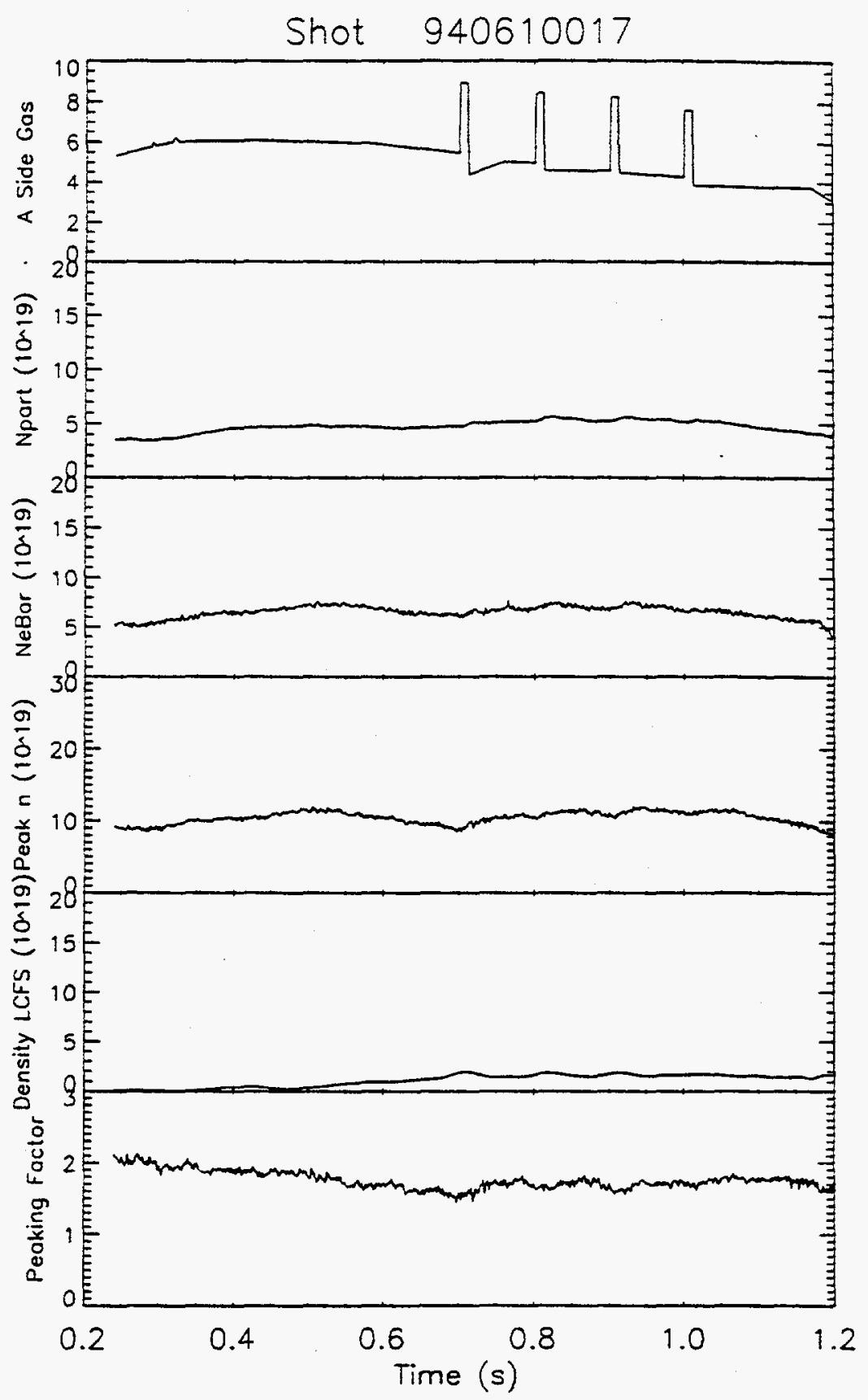

Figure 6.4: Standard outputs of inversion analysis: particle inventory, peak density, line averaged and peaking factor. 


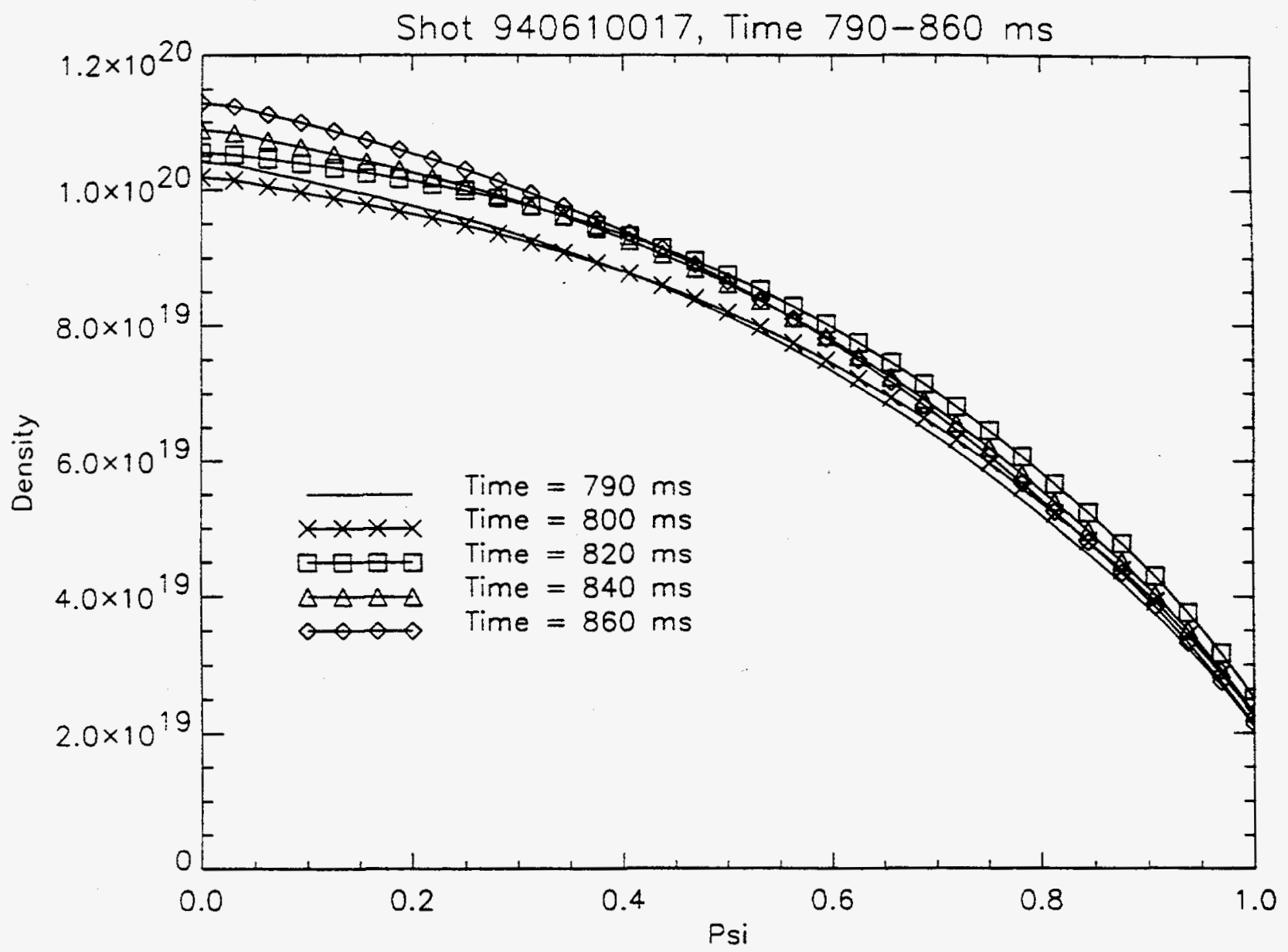

Figure 6.5: Time evolution of density profile during a gas puff. 
When the edge density was either too high or too low, the set of smoothing parameters that would fit both the desired edge density value and the chord measurements was small. Even when there were allowable values of epsilon, these tended to be small so that the resulting profile was unphysical. Recall that an allowable value of epsilon is one in which the edge error is less than 0.1 and the $\chi^{2}$ error is less than 0.5 . This was the case for fig $6.6 \mathrm{a}, \mathrm{d}$, and e. When the edge density was in the expected range, both the edge density and the chords were easily fit. The range of epsilon values was substantially larger as can be seen in figures $6.6 \mathrm{~b}$ and $\mathrm{c}$. In these cases, a relative evaluation of which edge density was better could be made. For this shot, an edge value of $2.5 \times 10^{19} \mathrm{~m}^{-3}$ was judged as best estimate. The corresponding range of epsilons was 0.9 to 1.4. A final value of 1.1 was chosen since it represented a minimum in the chi-squared error.

\subsubsection{Determination of the Edge Density}

An important input into the inversion was the magnitude of the density at the

LCFS. As seen in chapter 4, the profile is sensitive to this value although it is not sensitive to the shape of the edge region. Several different measurements were used to give a best estimate of the edge density. Table 6.1 compares the results from the model based on the divertor and limiter probes, the estimation of epsilon, and the reflectometer.

The agreement between the probe model, the epsilon analysis and the reflectometer was quite good. Between the probes and the epsilon analysis there was only one case in which the estimates vary more than $25 \%$. Preliminary results from the reflectometer showed that for the lower density shots, there was reasonable agreement with the epsilon analysis. Figure 6.7 shows that the predicted reflectometer signals based on the edge density estimated by the $\varepsilon$ analysis agrees quite well with the actual measurements. If an incorrect edge density is chosen, $100 \%$ errors can occur. (see figure 6.8) Thus, the reflectometer was useful to validate the estimates from the other edge 
a)

SHOT NUMBER 940610015

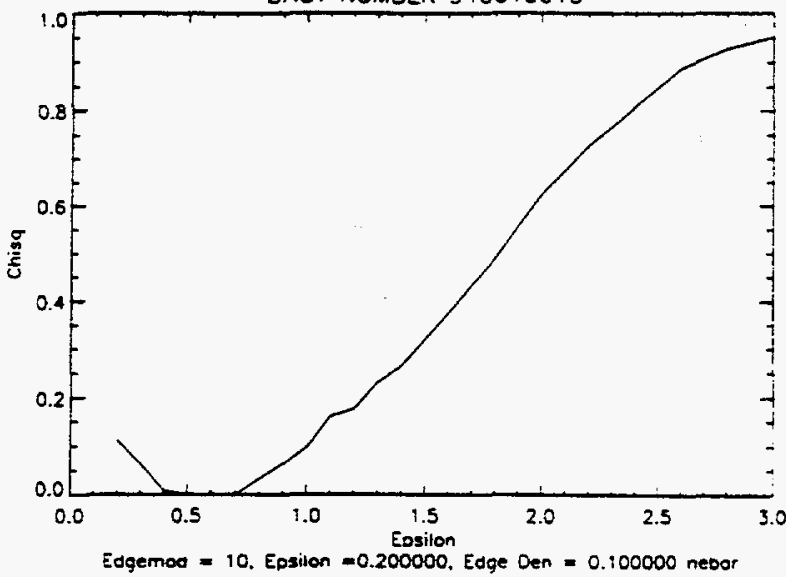

b)

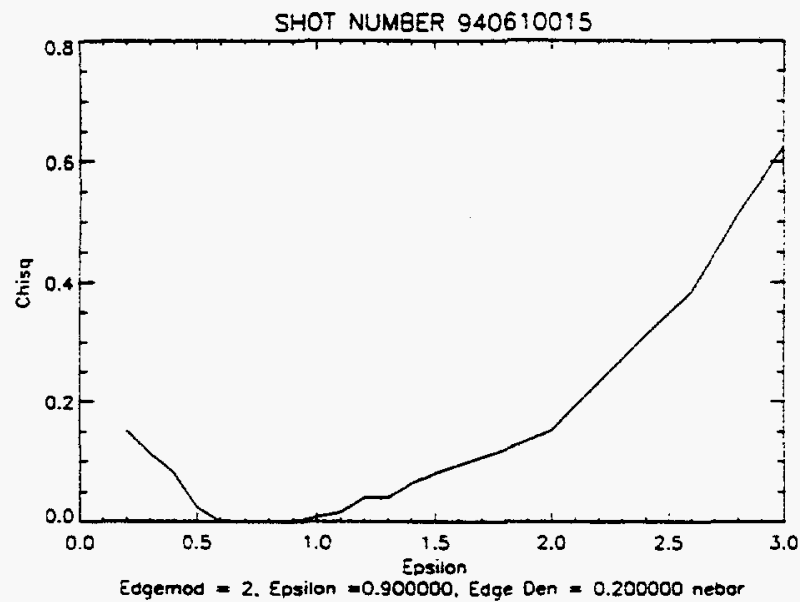

c)

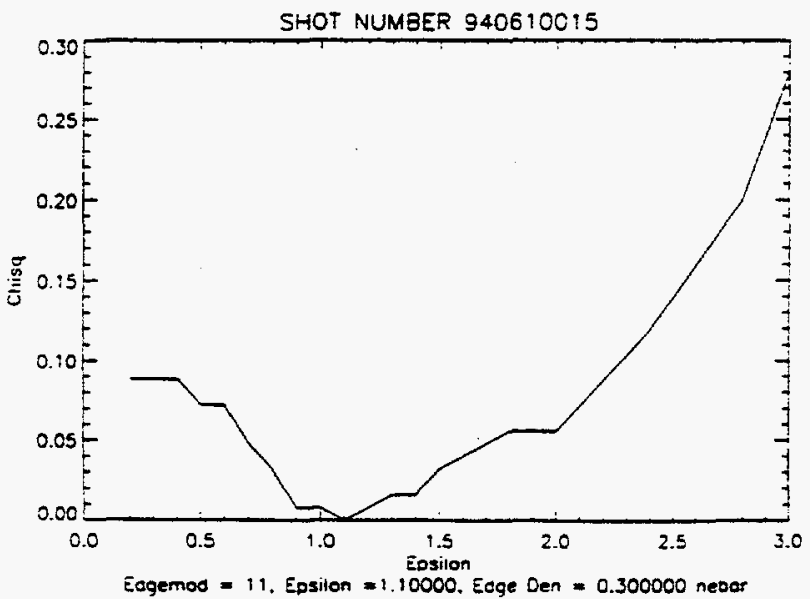

SHOT NUMBER 9405:0015
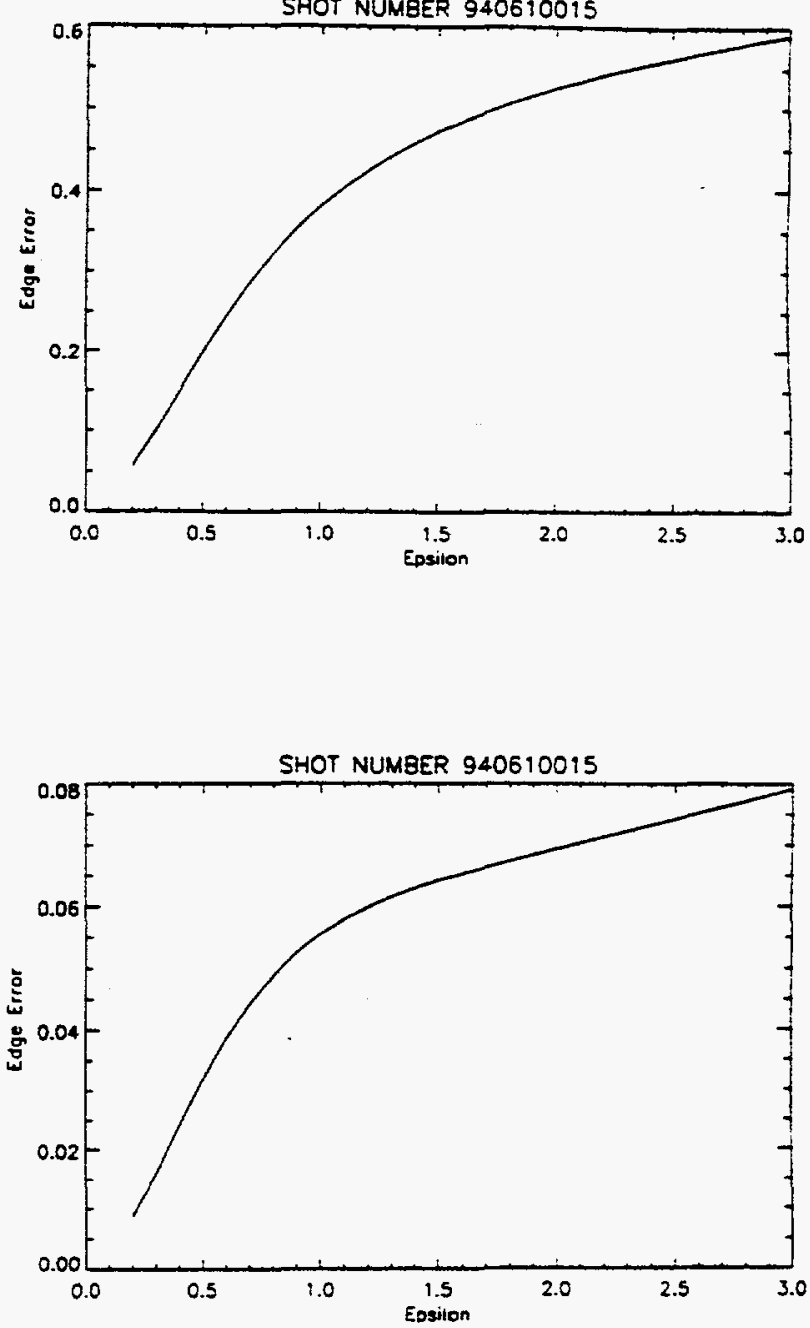
d)
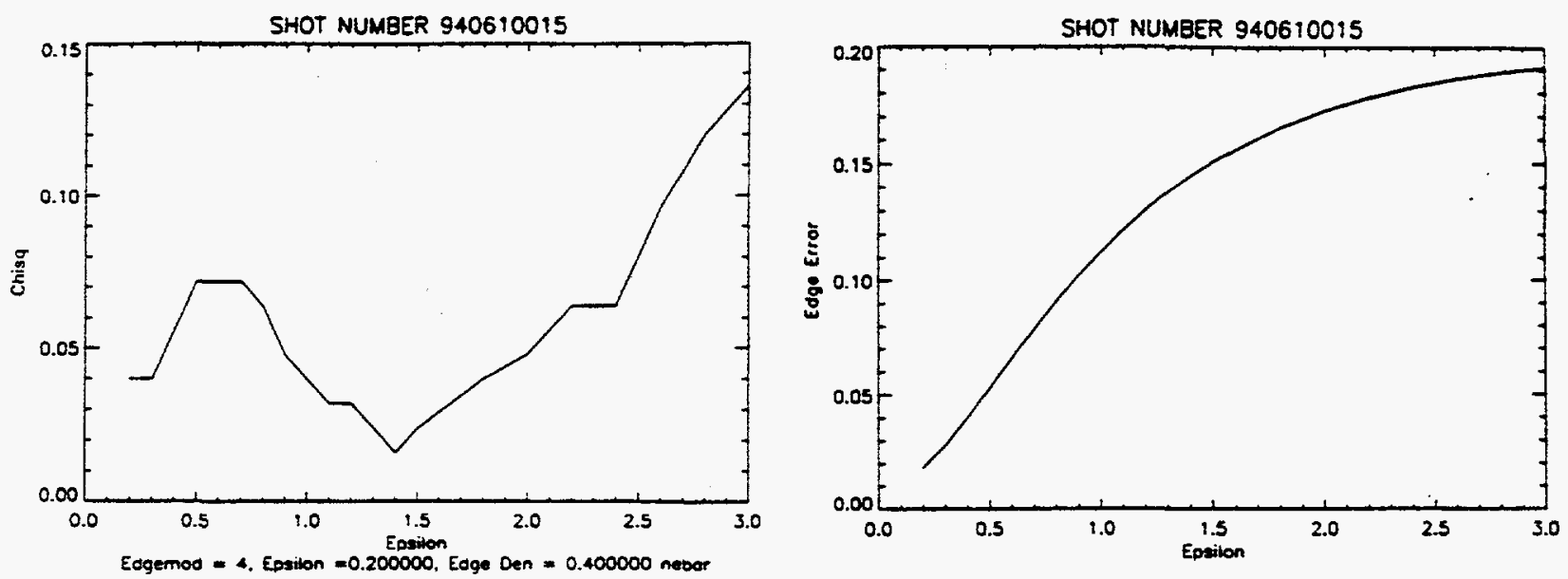

e)
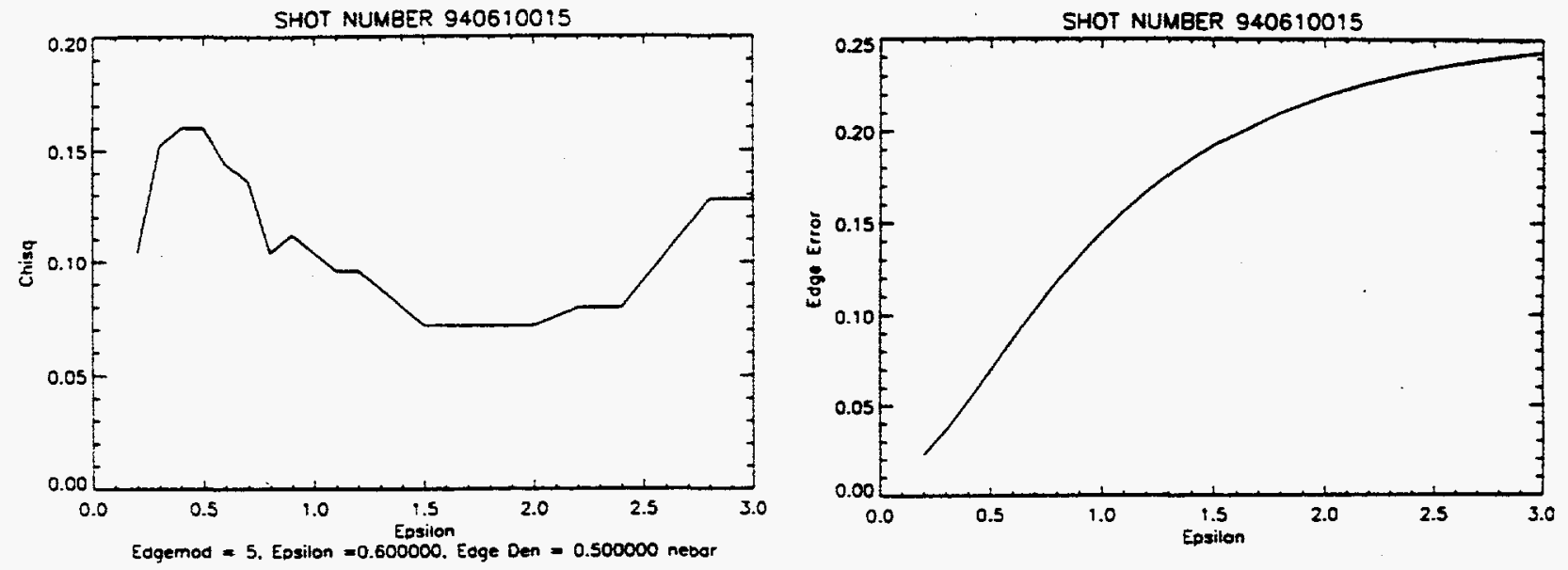

Figure 6.6: Errors from epsilon analysis for inversions with different edge densities: a) 0.1 $\bar{n}_{e}$, b) $0.2 \bar{n}_{e}$, c) $0.3 \bar{n}_{e}$, d) $0.4 \bar{n}_{e}$, and e) $0.5 \bar{n}_{e}$. Allowable epsilons are given by edge errors $<0.1$ and chisq errors $<0.05$. 
models. In general, the range of allowable edge densities from each method of analysis was comparable.

\begin{tabular}{|c|c|c|c|}
\hline Shot & Epsilon & Probes & Reflectometer \\
\hline & $10^{19} \mathrm{~m}^{-3}$ & $10^{19} \mathrm{~m}^{-3}$ & $10^{19} \mathrm{~m}^{-3}$ \\
\hline 940610005 & 3.9 & - & 2.6 \\
\hline 940610006 & 3.6 & - & - \\
\hline 940610007 & 3.1 & - & $1.0-3.1$ \\
\hline 940610009 & 2.5 & - & 1.7 \\
\hline 940610014 & 2.5 & 2.0 & 1.7 \\
\hline 940610015 & 2.5 & 1.3 & $0.9-1.7$ \\
\hline 940610016 & 1.4 & 1.7 & 1.5 \\
\hline 940610017 & 1.4 & 1.2 & 0.7 \\
\hline 940610023 & 2.9 & - & - \\
\hline 940610024 & 4.9 & 4.2 & - \\
\hline 940610025 & 5.0 & 5.0 & - \\
\hline 940610028 & 3.0 & 3.0 & - \\
\hline
\end{tabular}

Table 6.1: Estimates of the edge density at the LCFS as calculated from several sources.

By no means were these models exact and a calibrated reflectometer, edge Thomson Scattering measurement, or an edge interferometer chord is still necessary to pinpoint the edge density. However, consistent independent estimates gave confidence that the best estimate of the edge density was acceptable and can be used for the density inversion and transport analysis. 

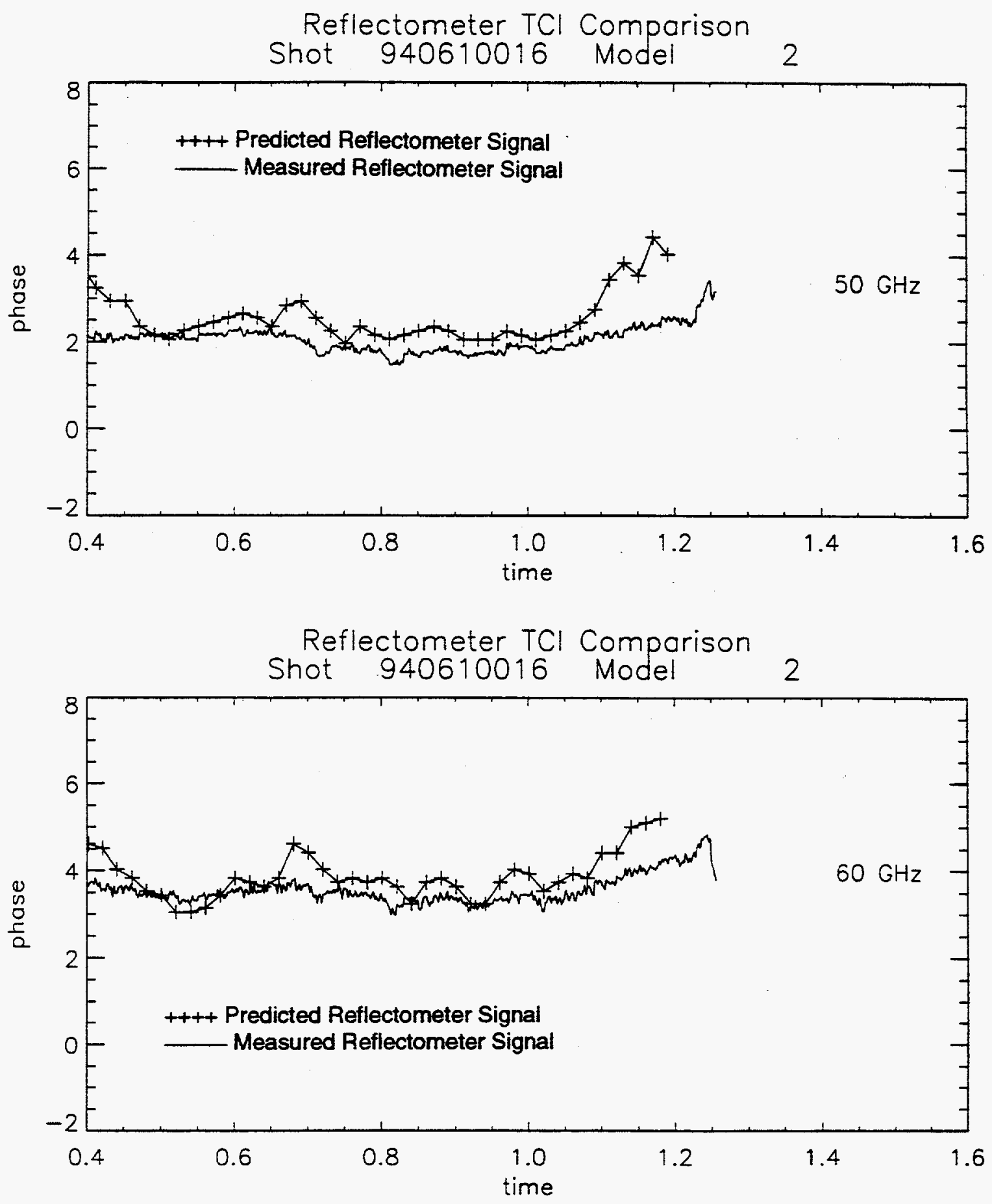

Figure 6.7: Comparison of predicted and measured reflectometer signals for the best estimate of the edge density from the epsilon analysis. 

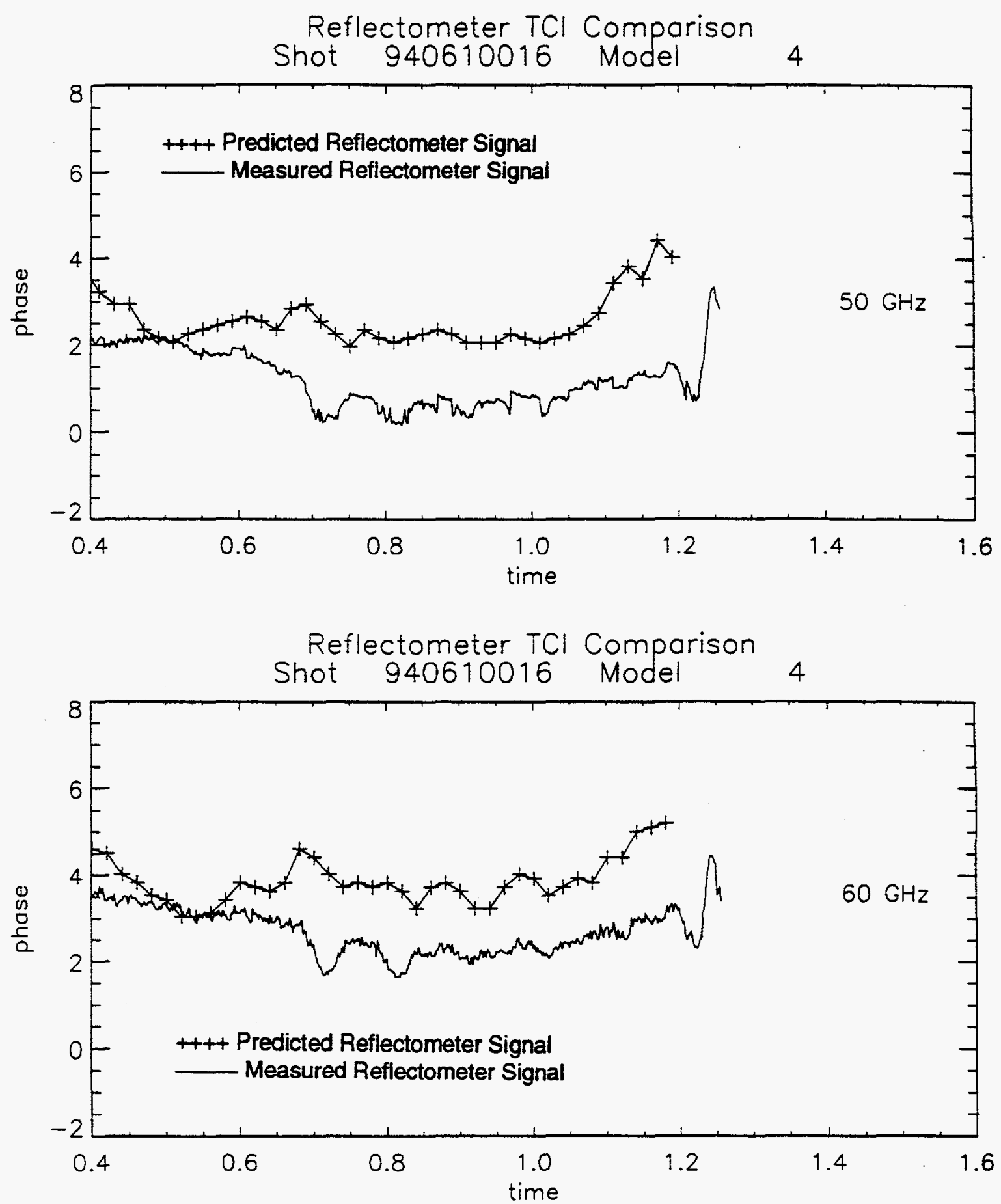

Figure 6.8: Comparison of predicted and measured reflectometer signals for the 2 times the best estimate of the edge density from the epsilon analysis. 


\subsection{Results from the Regression Analysis}

The regression analysis was applied to the inverted profiles to yield the transport coefficients. The coefficients were determined for the inversions using the best estimate of the edge density from the epsilon analysis.

The profiles of both $\mathrm{D}(\mathrm{r})$ and $\mathrm{V}(\mathrm{r})$ show an increasing value of the coefficient with increasing radius. The typical behavior of the coefficients can be seen in fig. 6.9. The shape of the profile agrees with those found by Efthimion, Marinak and Gentile [74, 105, $106,108]$. The increasing profile is consistent with equilibrium profile shapes. $\mathrm{D}$ and $\mathrm{V}$ values are typically small on axis since in the core the density gradients are small while the density is the greatest. As the radius increases, the density decreases and the gradients increase, resulting in an decrease of the scale length. An increase in both $D$ and V but with $V$ increasing faster will allow the density scale length and the $D / V$ ratio to remain equal in regions which are source-free.

The value of the diffusion coefficients also decreases with the background density. This is illustrated in figure 6.10 where shots of similar background density have been averaged. As the density increases, both the $\mathrm{D}$ and $\mathrm{V}$ profiles fall. For a $50 \%$ rise in the line average density, a drop by a factor of 3 occurs. At the highest densities of $\bar{n}_{e}>1.0 \times$ $10^{20} \mathrm{~m}^{-3}$, the measured values at $\Psi$ equal to 0.5 are extremely low with $\mathrm{D}<0.05 \mathrm{~m}^{2} / \mathrm{s}$ and $\mathrm{V}<0.5 \mathrm{~m} / \mathrm{s}$.

\subsubsection{Sensitivity of the Regression Analysis}

The two greatest sources of systematic error are the estimates of the edge density and the selection of the smoothing parameter. Of these two, the regression analysis is clearly more sensitive to the value of the edge density. In fig. 6.11 , the regressed transport coefficients for a shot are presented with the edge density ranging from 0.1 to $0.5 \bar{n}_{e}$. The value of the coefficients clearly fall as the edge density rises. This behavior is due to the flattening of the density profile as the edge density rises; in chapter 4 , the 

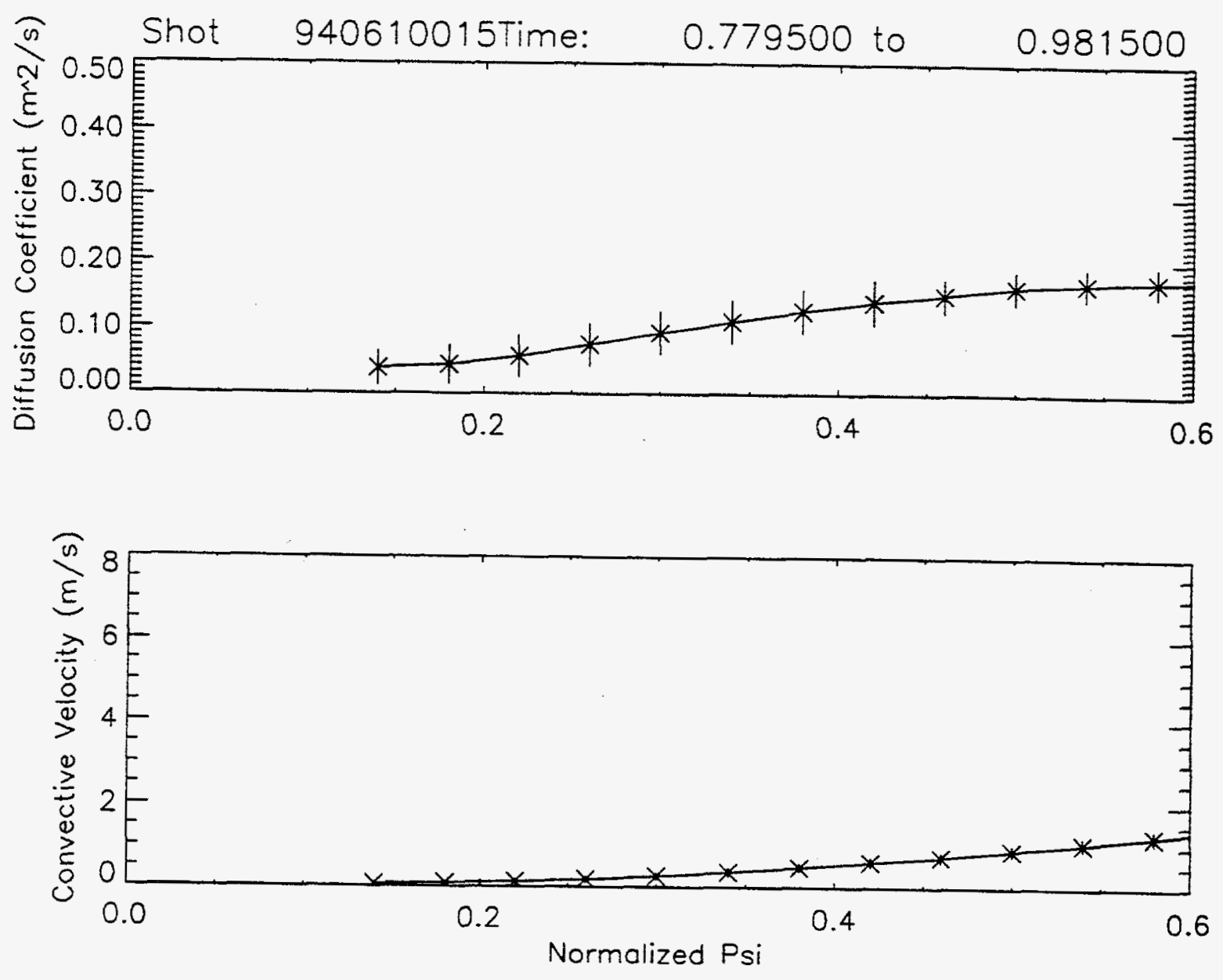

Figure 6.9: Transport coefficient profiles from regression. 

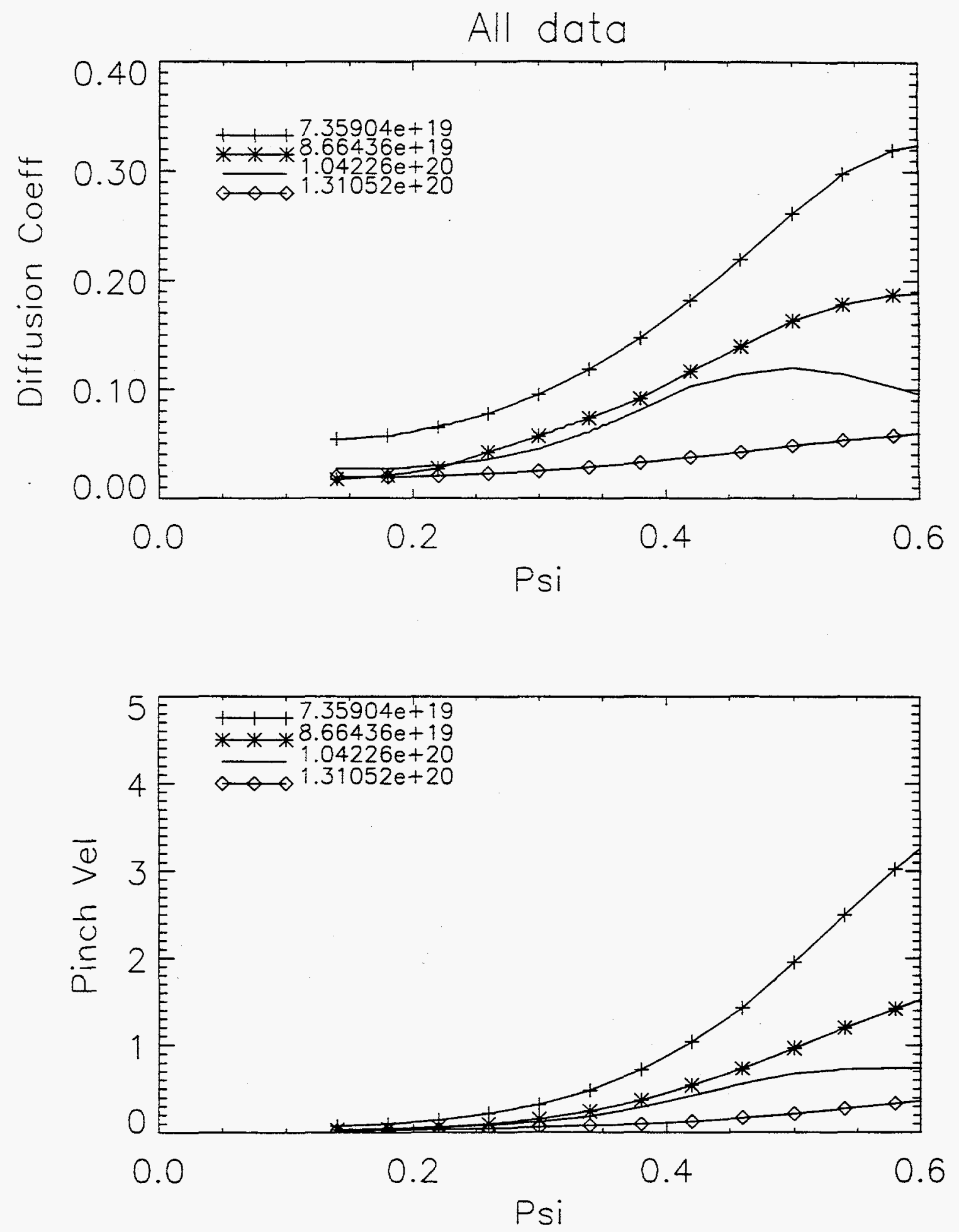

Figure 6.10: Comparison of transport coefficient profiles for line averaged densities of $7.4 \times 10^{19}$ to $1.31 \times 10^{20} \mathrm{~m}^{-2}$. 
profiles were shown to become flatter and even hollow. To remain in agreement with the increasing density length scale, the coefficients must become smaller, with $V$ decreasing more than D. The resulting V/D ratios confirm that this fall in the coefficient values is the result of profile shape changes. For the lower edge densities, the profiles are the most peaked and consistently, the V/D ratio is the greatest. This is seen in figure 6.12. It should also be noted that when the edge density is in the acceptable range as determined by the epsilon analysis, the variation of the transport coefficients decrease. The values of the coefficients become much more comparable at those densities. This is observed in fig 6.11 where the curves for an edge density of .2 and $.3 \bar{n}_{e}$ exhibit less variation between each other than between the other curves.

In chapter 4 , it was observed that the inversion is relatively insensitive to the smoothing parameter. As long as $\varepsilon$ was in the allowable range, the profiles show little change in shape but there is approximately a $5 \%$ change in the peak density. Figure 6.13 shows the variation in the corresponding coefficient profiles as derived from the regression analysis. There are small differences in the diffusion coefficients but this error is less than that due to an error in the edge density.

When the regression is applied, there is an implicit assumption that during the analysis period the transport can be explained by a temporally constant value. To test this assumption, the regressed diffusion coefficients were used to reconstruct the predicted change in particle inventory inside a flux surface. This was then compared to the actual measured change as calculated from the density profiles. For a good shot, in which the puff was sufficiently large to affect the profiles at the core, reasonable agreement was observed between the reconstructed and measured quantities. Figure 6.14 shows the time rate of change of the particle inventory over three puffs. The regression has the same general shape as the measured time derivative but fails to reproduce the correct amplitude. This indicates that not all the profile changes have been captured by the density inversion. 
Shot 940610017

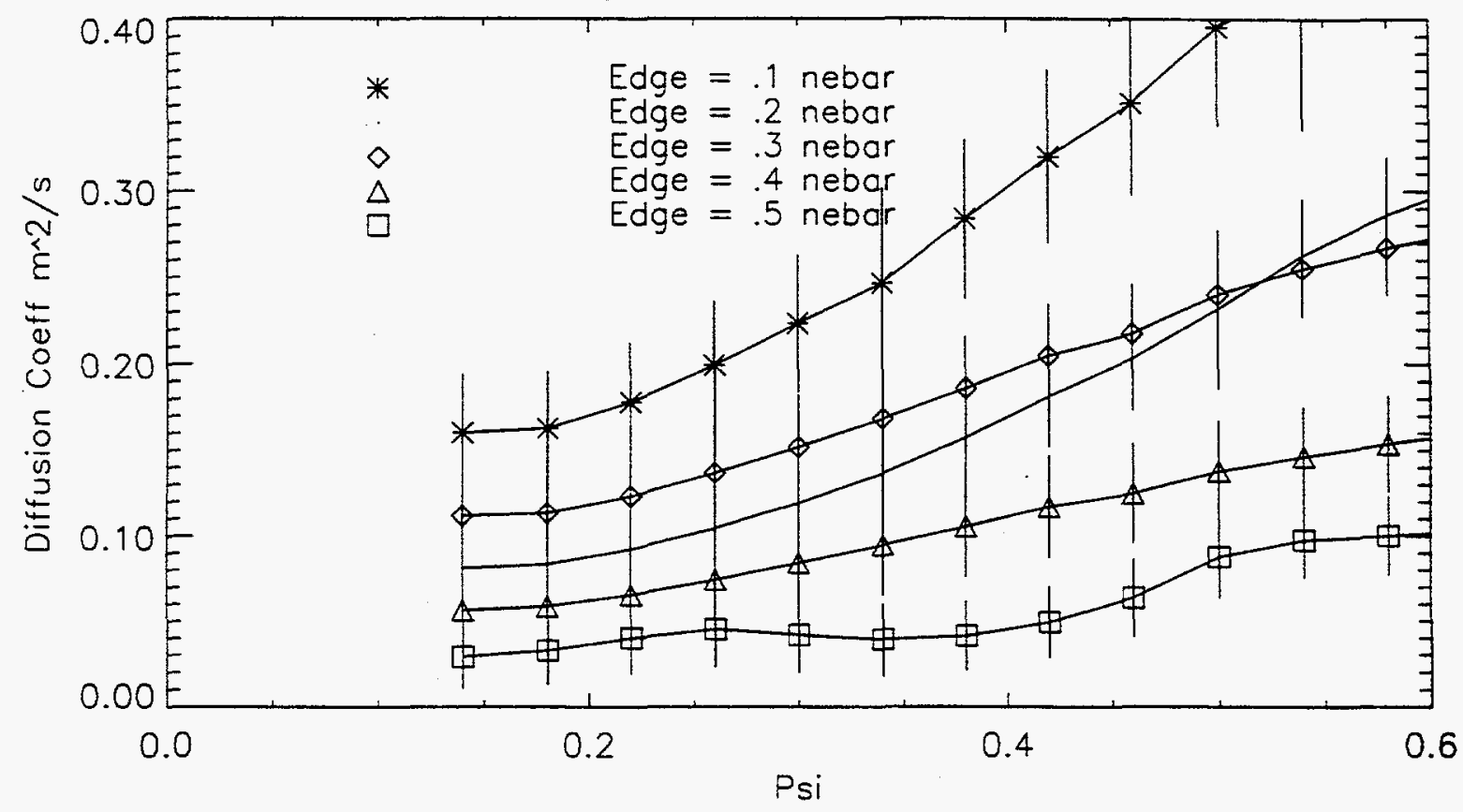

Shot 940610017

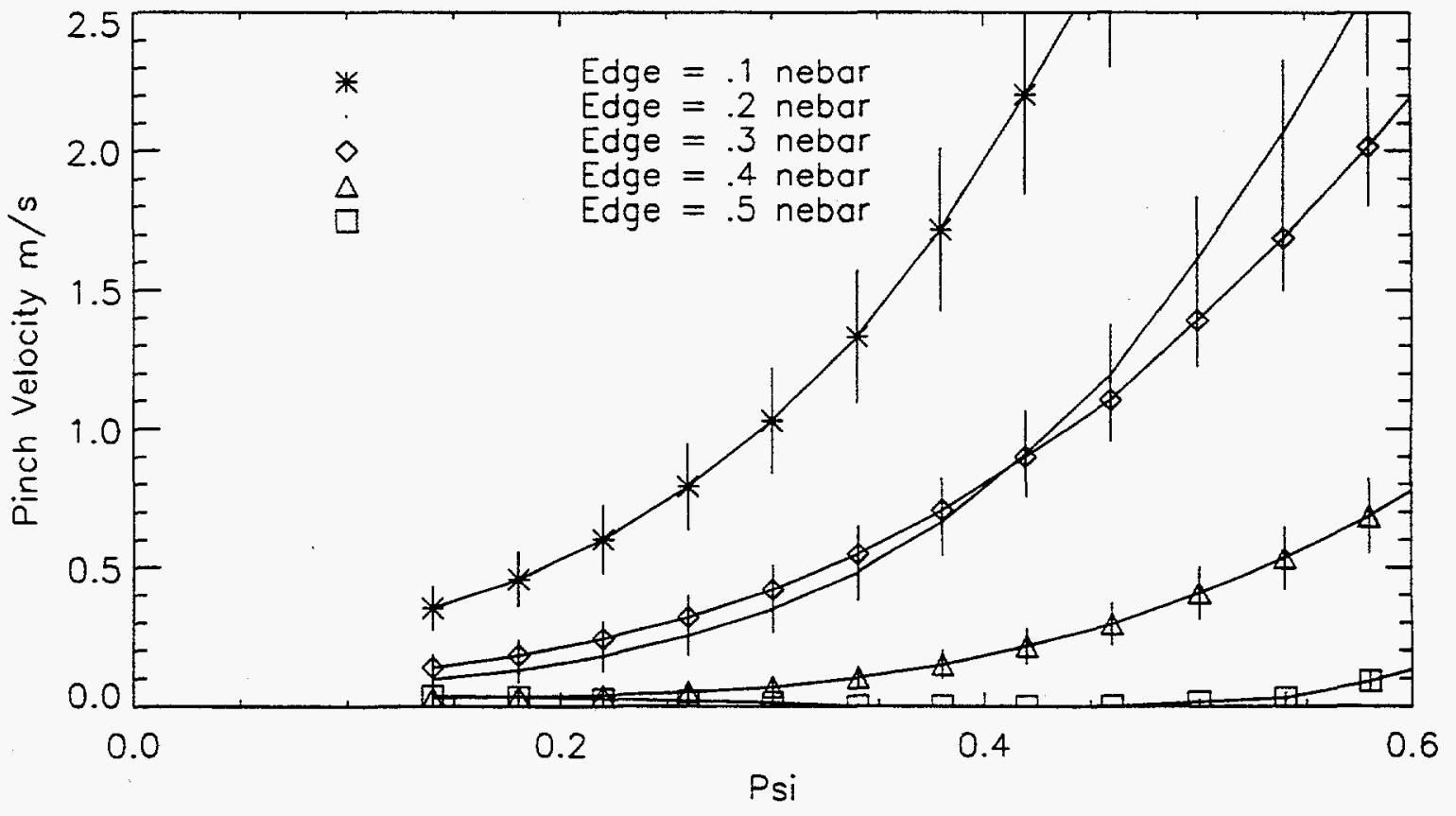

Figure 6.11: Variation of regressed transport coefficients with edge density. 


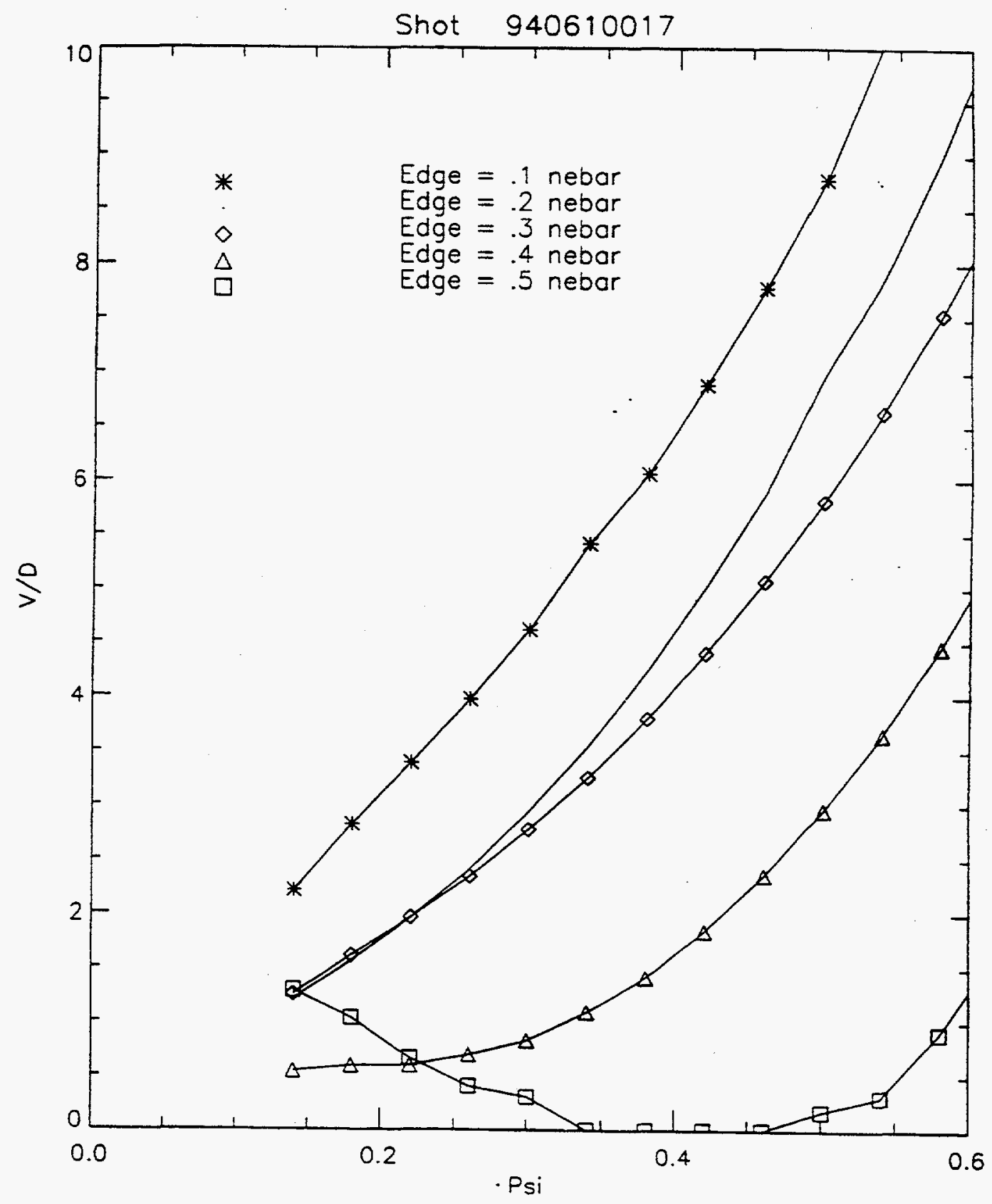

Figure 6.12: Variation of regressed V/D ratio with edge density. 
Shot 940610015
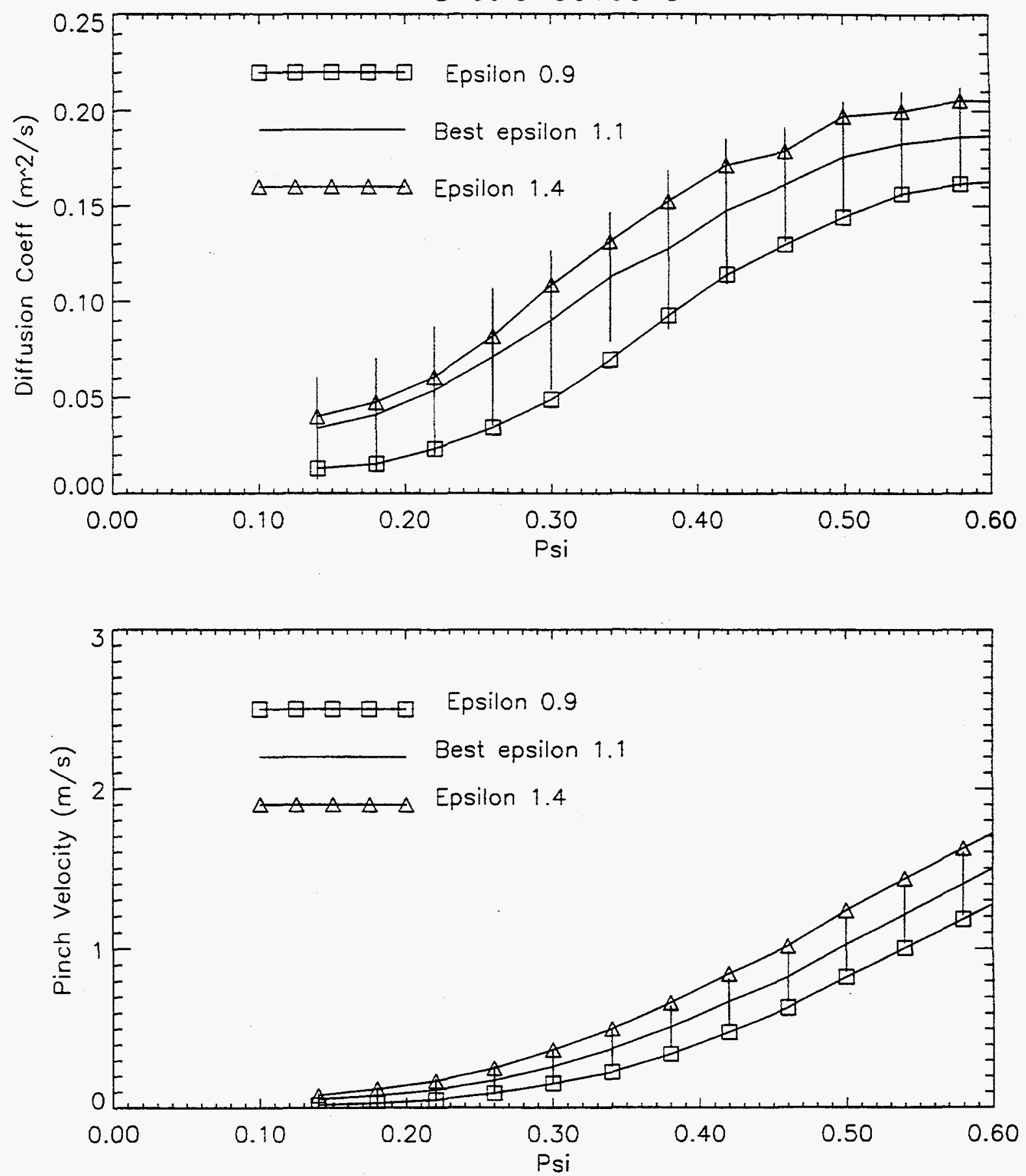

Figure 6.13: Variation of transport coefficients profiles with smoothing weight, $\varepsilon$. 


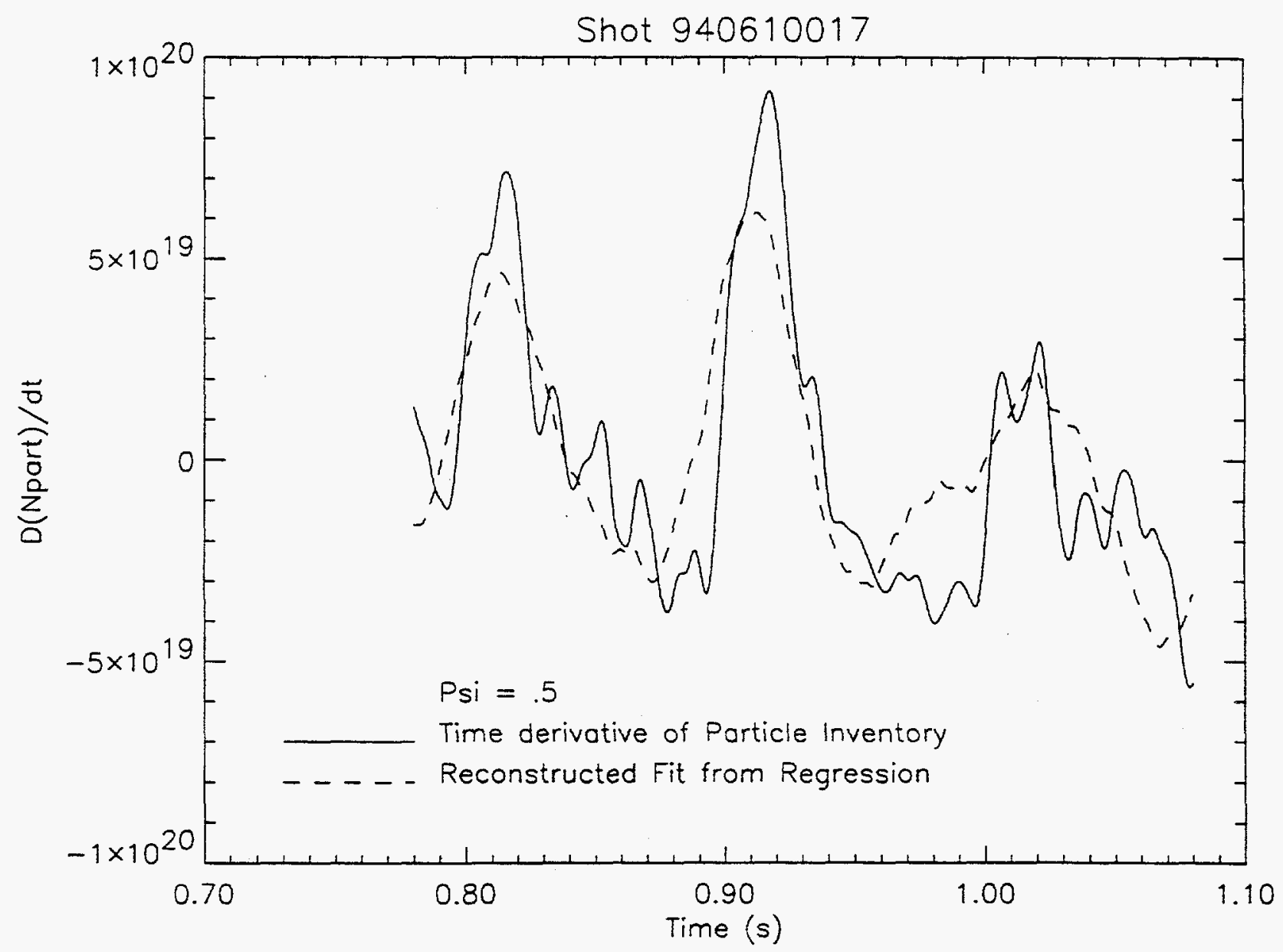

Figure 6.14: Comparison of reconstructed time derivative of the particle inventory from the regression and the measured time derivative. 
An additional test of the assumption can be made by taking regressions over a smaller time interval. The choice of the time window is an important issue when doing the regressions. Typically, the time interval was taken to incorporate 2 or 3 puffs. More importantly, the puffs were chosen so that there was both a noticeable rise and decay of the density. Often this meant discarding the first puff since the density did not decay but remained high. This indicated that the plasma had not yet reached equilibrium after diverting. Figure 6.15 shows the regressed coefficients from the entire regression window and those from the individual regressions of each puff. Good agreement between all these regressions indicate that the assumption of time constant diffusion coefficients is valid.

In chapter 4 , it was shown that the inversions were insensitive to small radial translations of the plasma. This analysis was extended to the regression analysis. The result, as seen in Figure 6.16, is that the regressed transport coefficients are also insensitive to the shift. The variation is well within the error bars of the measured profiles and is not the largest source of systematic error.

\subsection{Results from the Simulation Analysis}

A numerical simulation as described in chapter 5 was also applied to the gas modulated shots. The resulting coefficient profiles agreed well with those from the regression. Figure 6.17 compares the regressed coefficient profile and the simulated profile for a typical shot. Agreement is quite good with the simulation falling within the error bars of the regression. Comparisons beyond $\Psi=0.6$ were not done since there are no phase measurements in that region. In fig 6.18, the results are compared for four shots, one at each of the densities shown in fig 6.9. The profiles are within the error bars of the regression for $\Psi<0.5$. The diffusion coefficients exhibit the same general shape and the same inverse dependence with the density. Comparisons of the simulation with measured quantities was also good. For each shot, the source terms were adjusted so that the total particle inventory was matched to the measured inventory to better than $2 \%$. Figure 6.19 
Shot 940610016
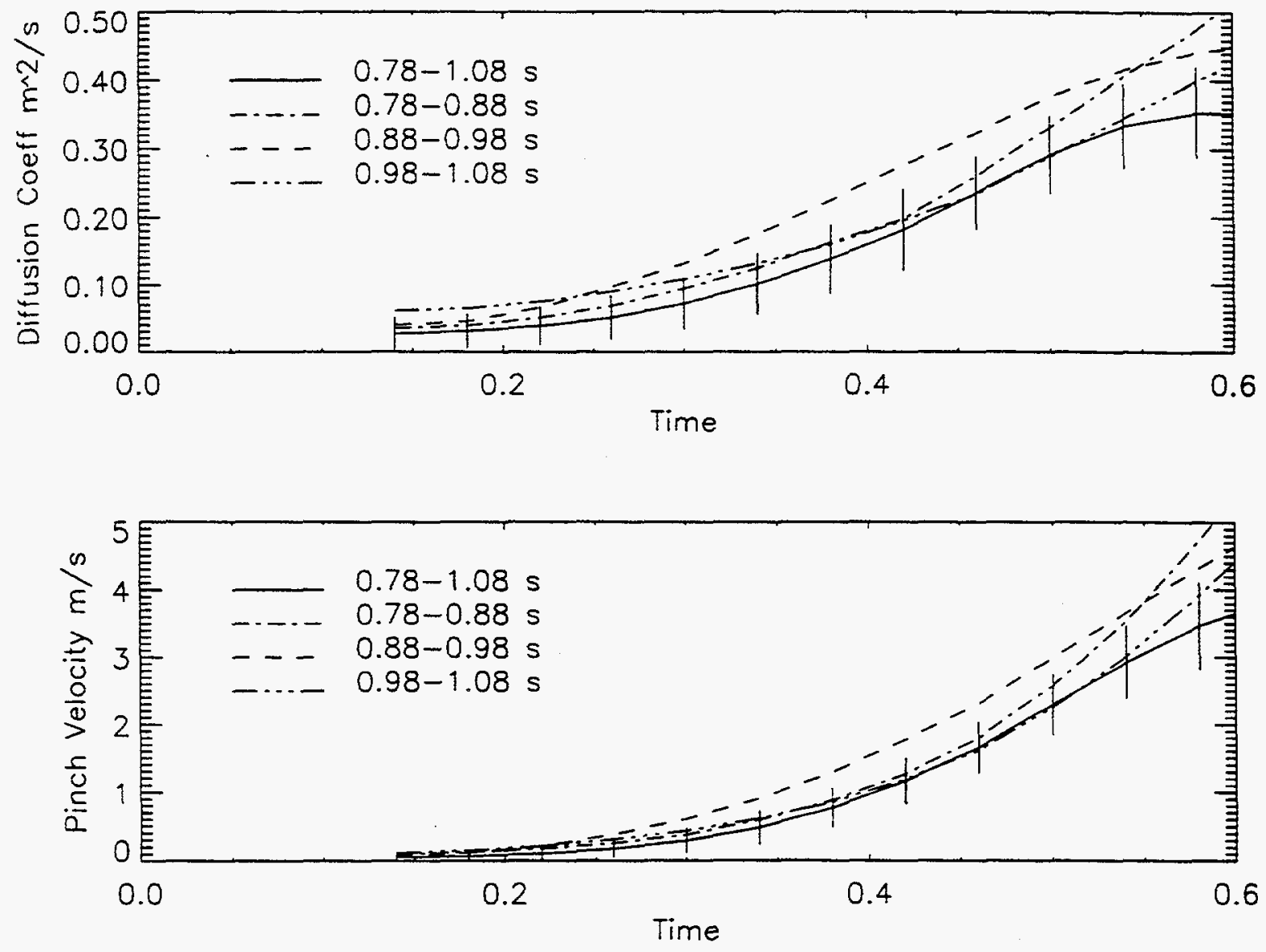

Figure 6.15: Comparison of regressed coefficient profiles for regressions over three puffs individually and over all three puffs simultaneously. 
Shot 940610017
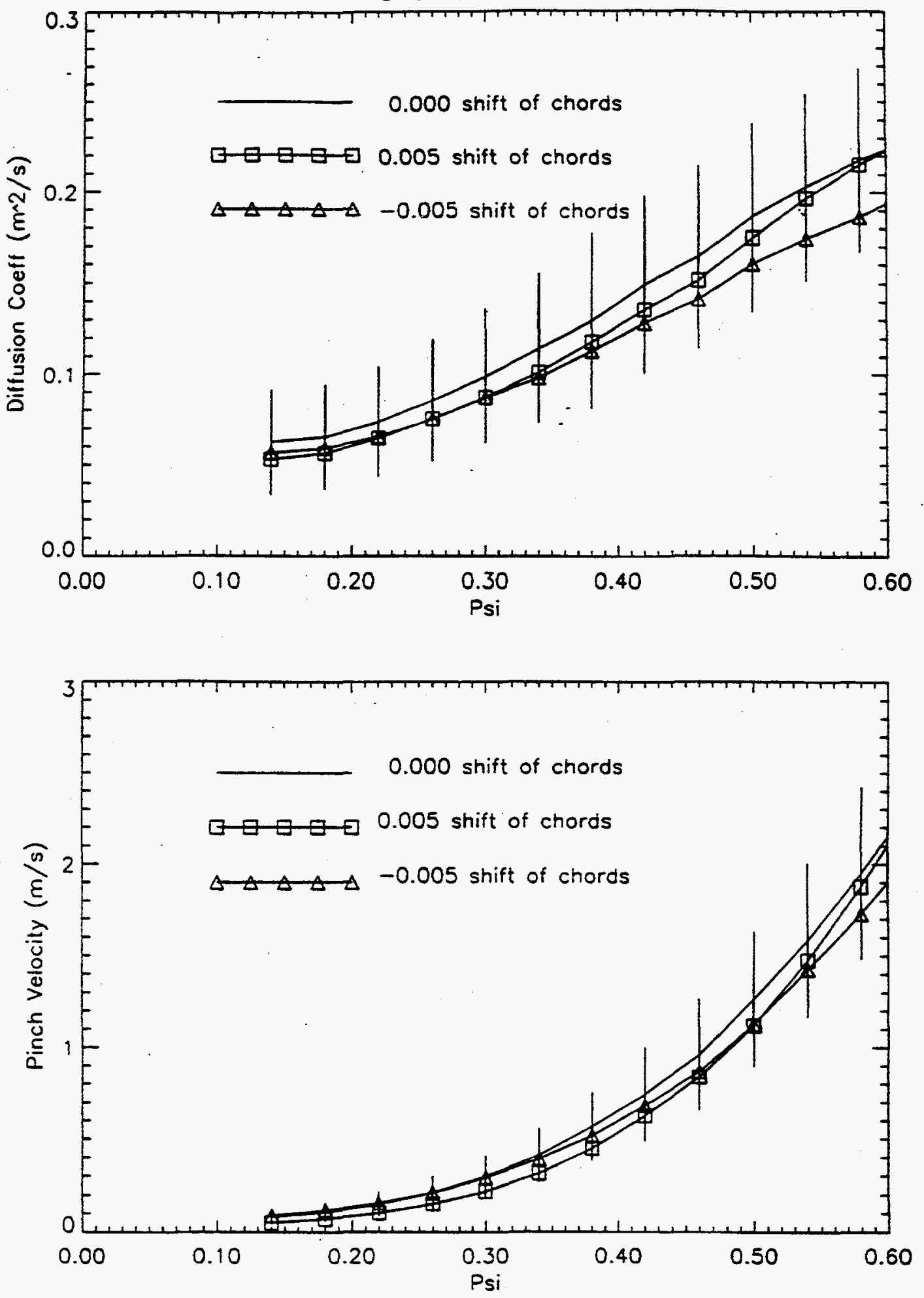

Figure 6.16: Variation of coefficient profiles with shifts in the magnetic geometry. 
SHOT NUMBER 940610015

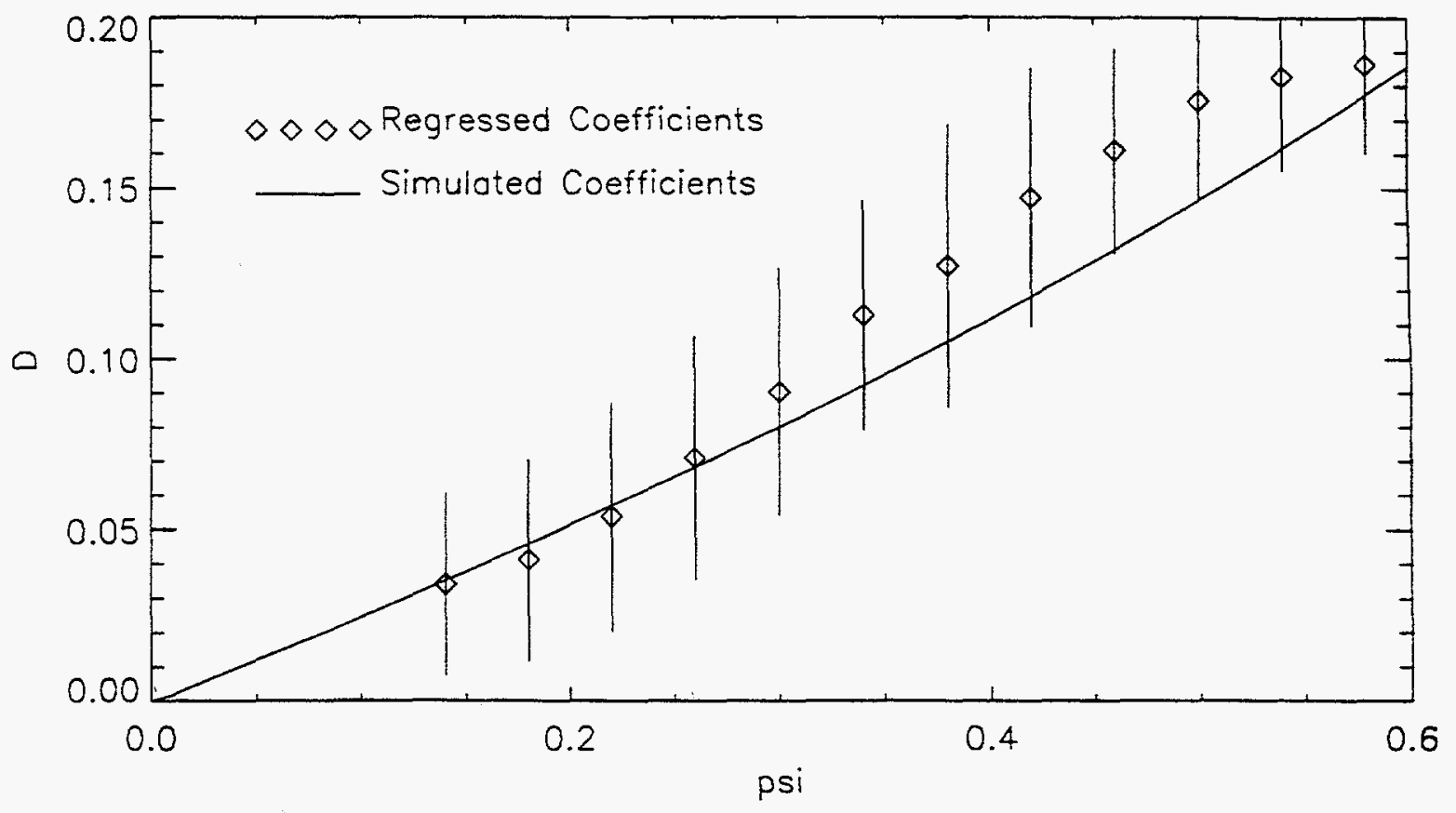

SHOT NUMBER 940610015

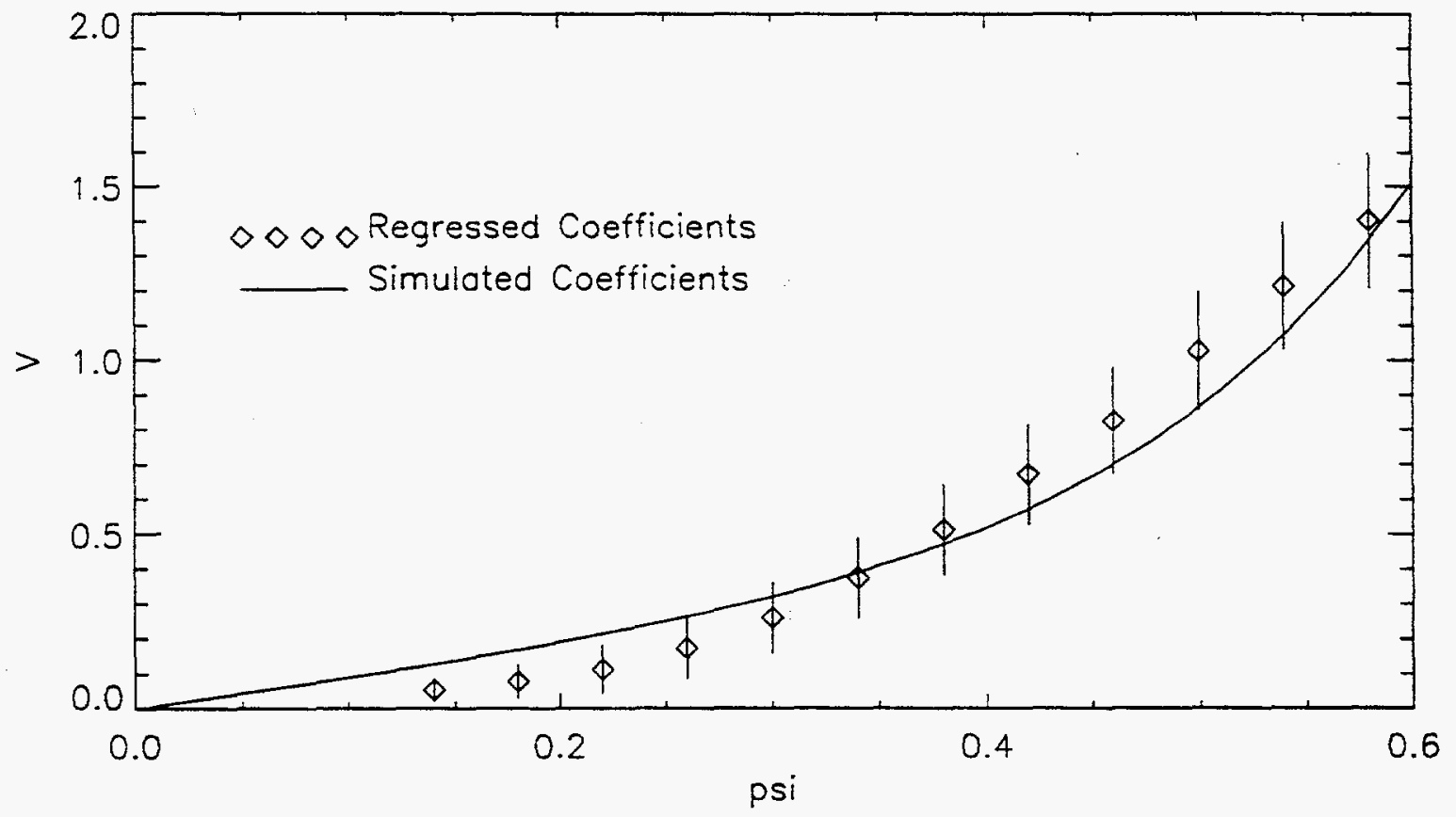

Figure 6.17: Comparison of transport coefficient profiles from the regression analysis and the numerical simulation. 

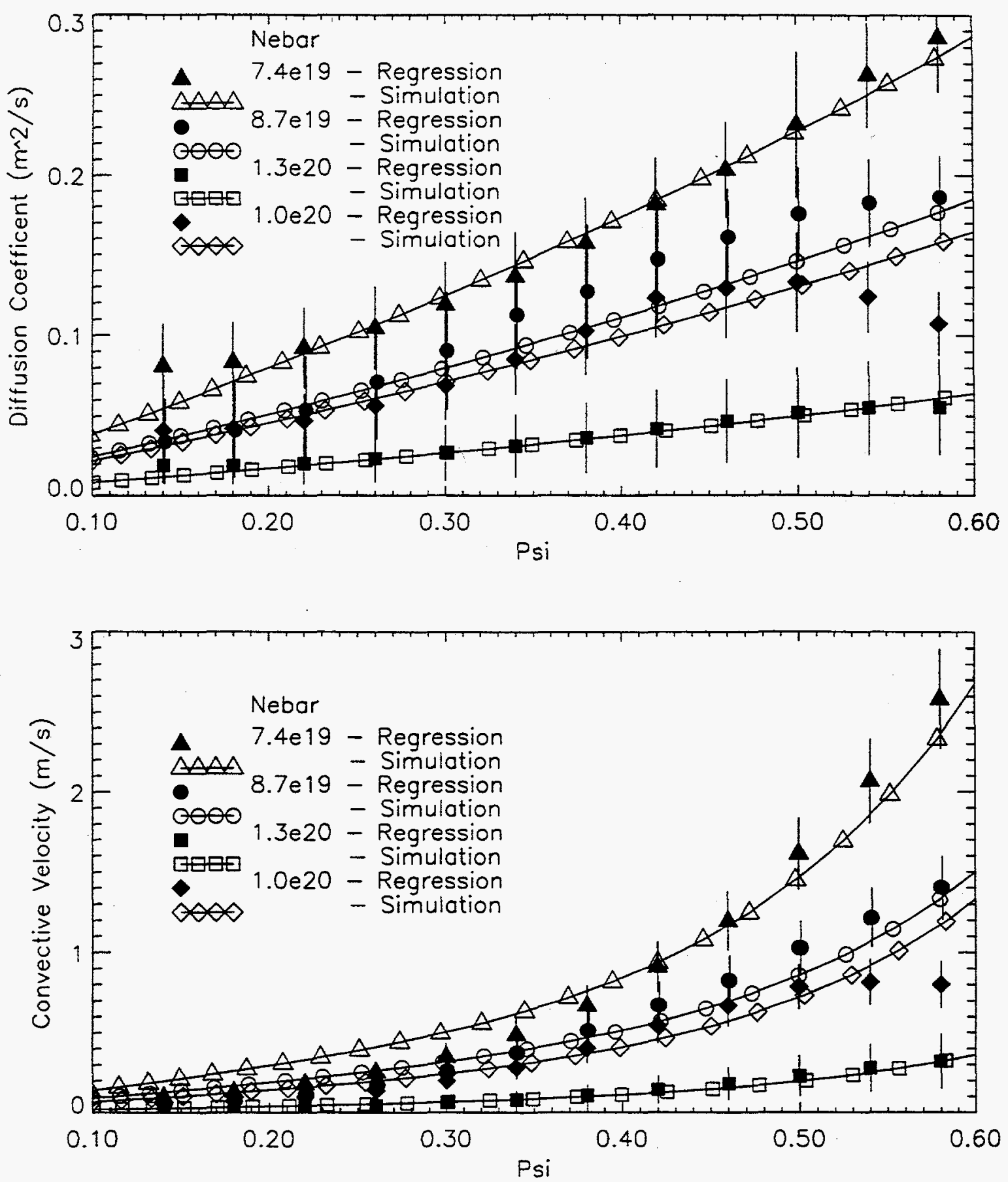

Figure 6.18: Comparison of transport coefficient profiles from the regression analysis and the numerical simulation for the same background densities as fig. 6.9 . 
shows the particle inventories and the fractional error of the simulation for a puff at 800 ms. The simulation was conducted for $100 \mathrm{~ms}$. Simultaneously, the phase measurements were also matched to within less than $2 \%$ error by adjusting the coefficient profiles. The match of the phase signal and the corresponding error is presented in fig 6.20 for a central, inner and outer chords.

\subsubsection{Sensitivity of the Numerical Simulation}

Because there are 6 free parameters when simulating the gas puff, two for each diffusion coefficient profile, the size of the background source and the size of the puff, it is necessary to investigate the uniqueness of the solution. In chapter 5 , it was noted that the system is overdetermined since there are 10 phase measurements to match. Thus, the solution would be a best fit to all 10 chords. Furthermore, since the profile shape restricts the V/D ratio, a match to the time histories during a gas puff should result in only one set of suitable profiles. Studies with the simulation have indicated that for different background source sizes, the coefficient profiles that satisfy the equilibrium profile shape, result in the same V/D profile. To test the sensitivity of the solution, the variation of the coefficients must be done in such a way that this V/D ratio is maintained. It is improper to vary only one of the coefficients at a time since this will change the V/D ratio and the resulting coefficients will not be consistent with the initial equilibrium profile.

In the simulation, a check of the best fit solution found that the resulting diffusion coefficients were close to their equilibrium values. Simulations with the diffusion coefficients perturbed by $\pm 10 \%$ from the best fit also resulted in reasonable fit to the equilibrium density profile. Thus, small changes in the coefficient profiles that maintain the V/D ratio are indistinguishable by looking at only the equilibrium profiles. However, when these same coefficient profiles were used to simulate the gas modulation, the time histories did not match. Figure 6.21 shows the simulations with the varied coefficients. Variation of the coefficients away from the best fit increases the relative errors in both the 
Shot 940610015

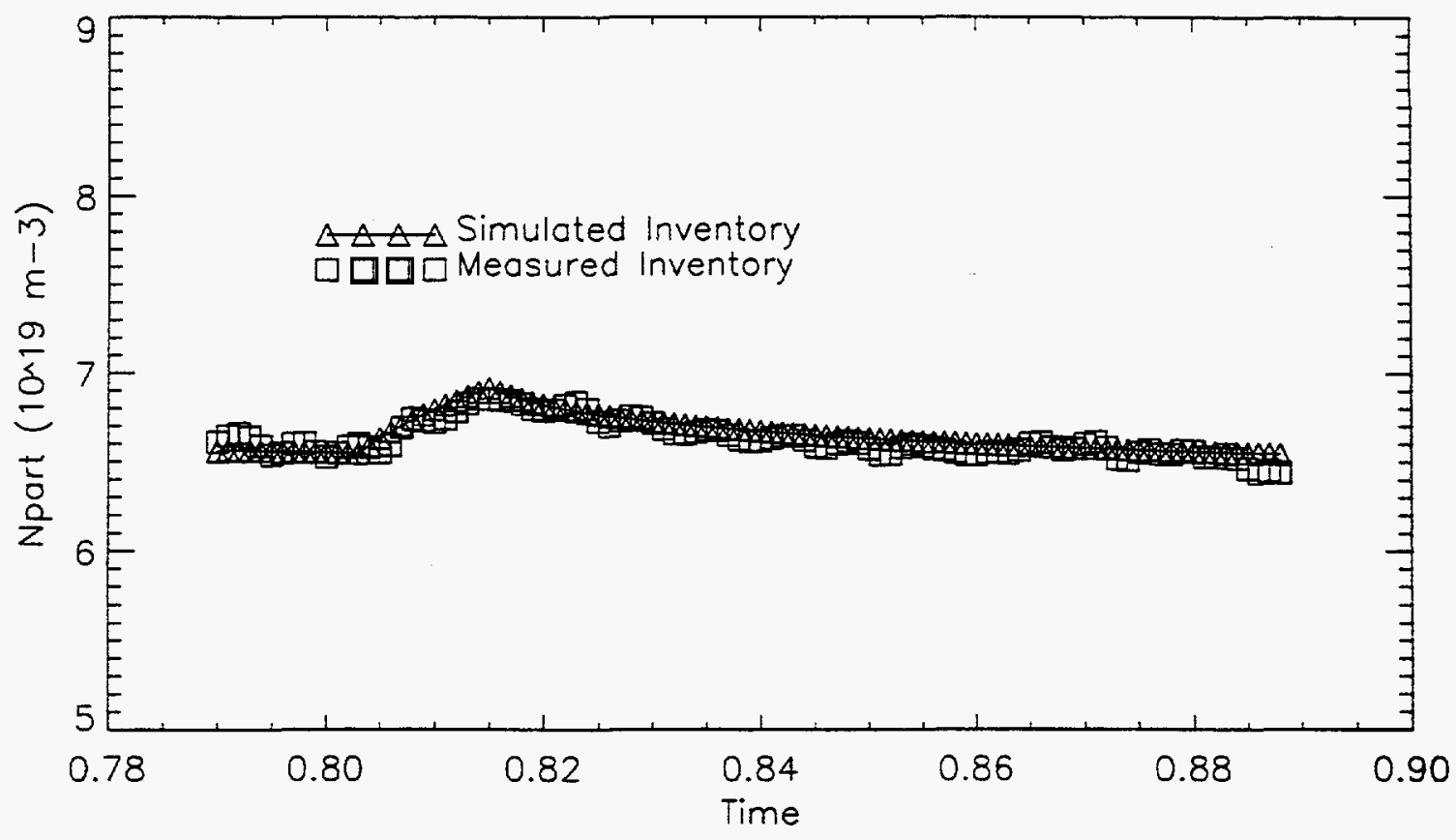

SHOT NUMBER 940610015

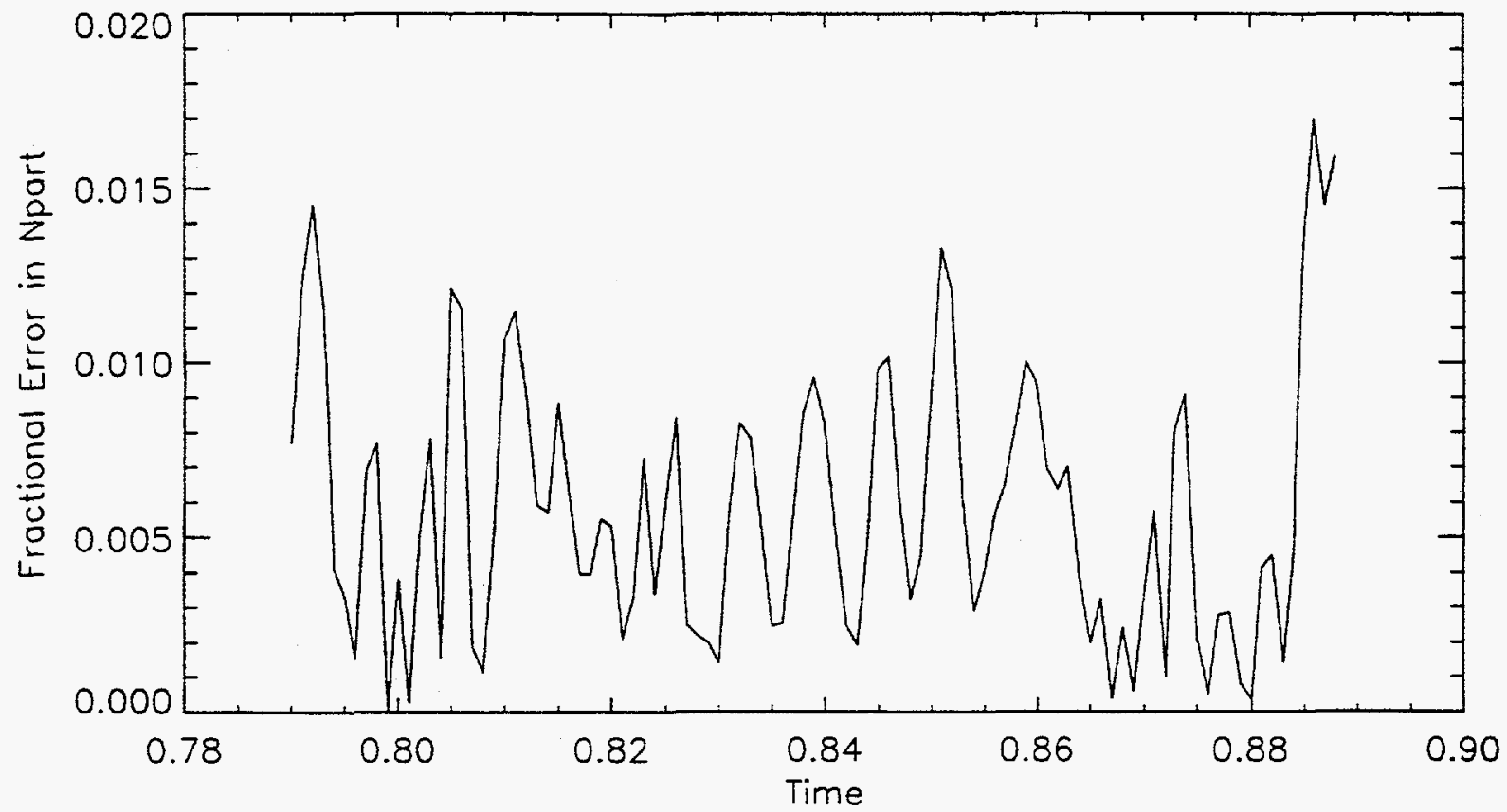

Figure 6.19: Comparison of simulated and measured particle inventory time histories. 
Shot 940610015 Chord at 0.655556

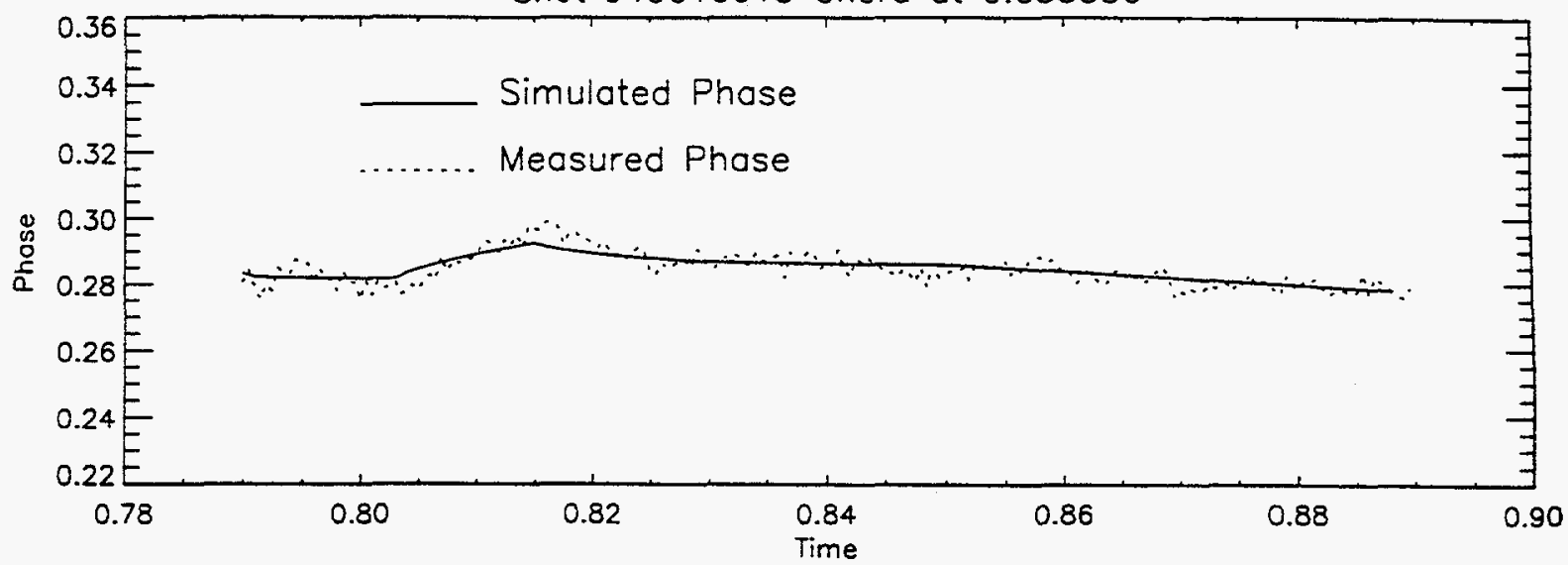

Chord at 0.708889

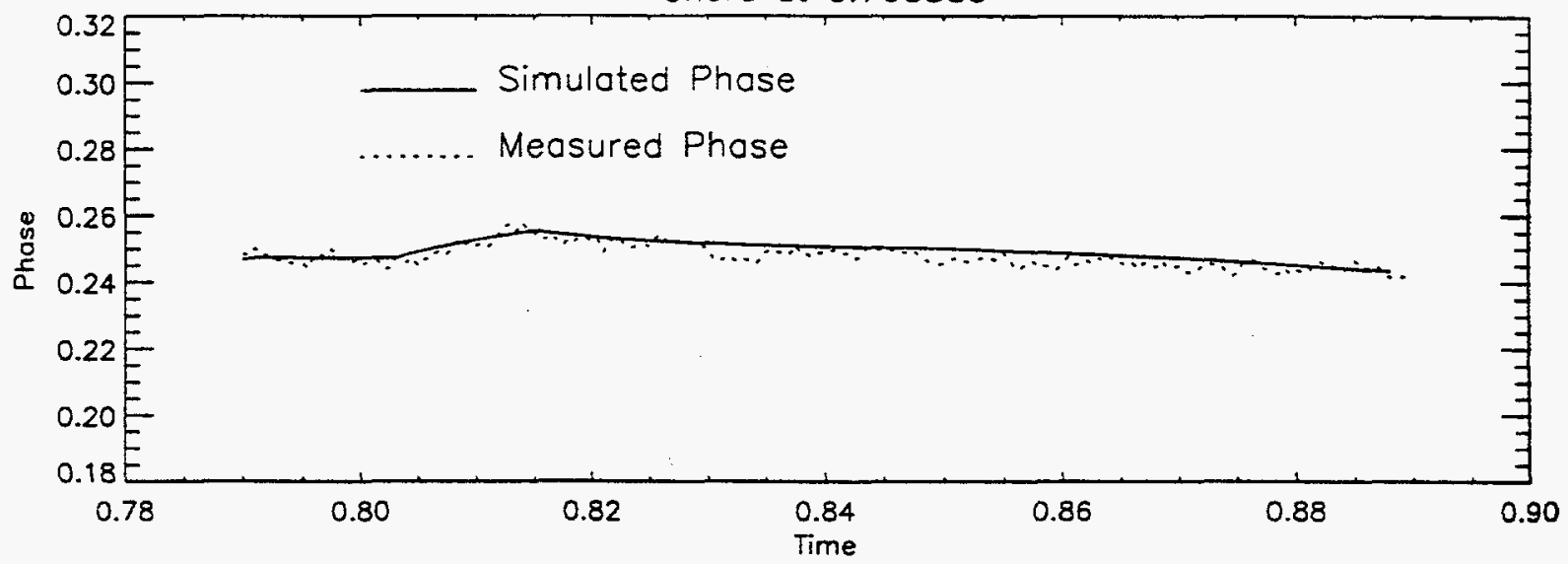

Chord at 0.780000

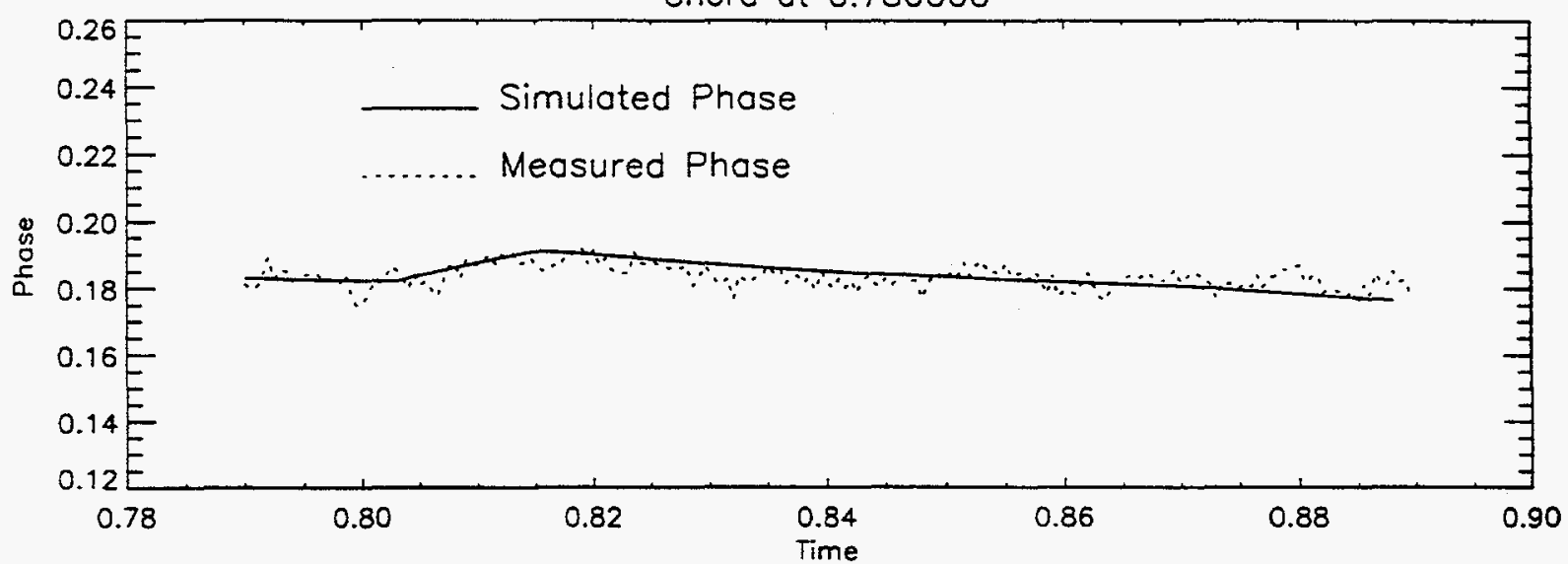

Figure 6.20: Comparison of the measured and simulated time histories of the interferometer signals. 
fits to the phase and total particle inventory by a factor of 2 . Thus, the simulation is sensitive to changes in the profiles at the $10 \%$ level.

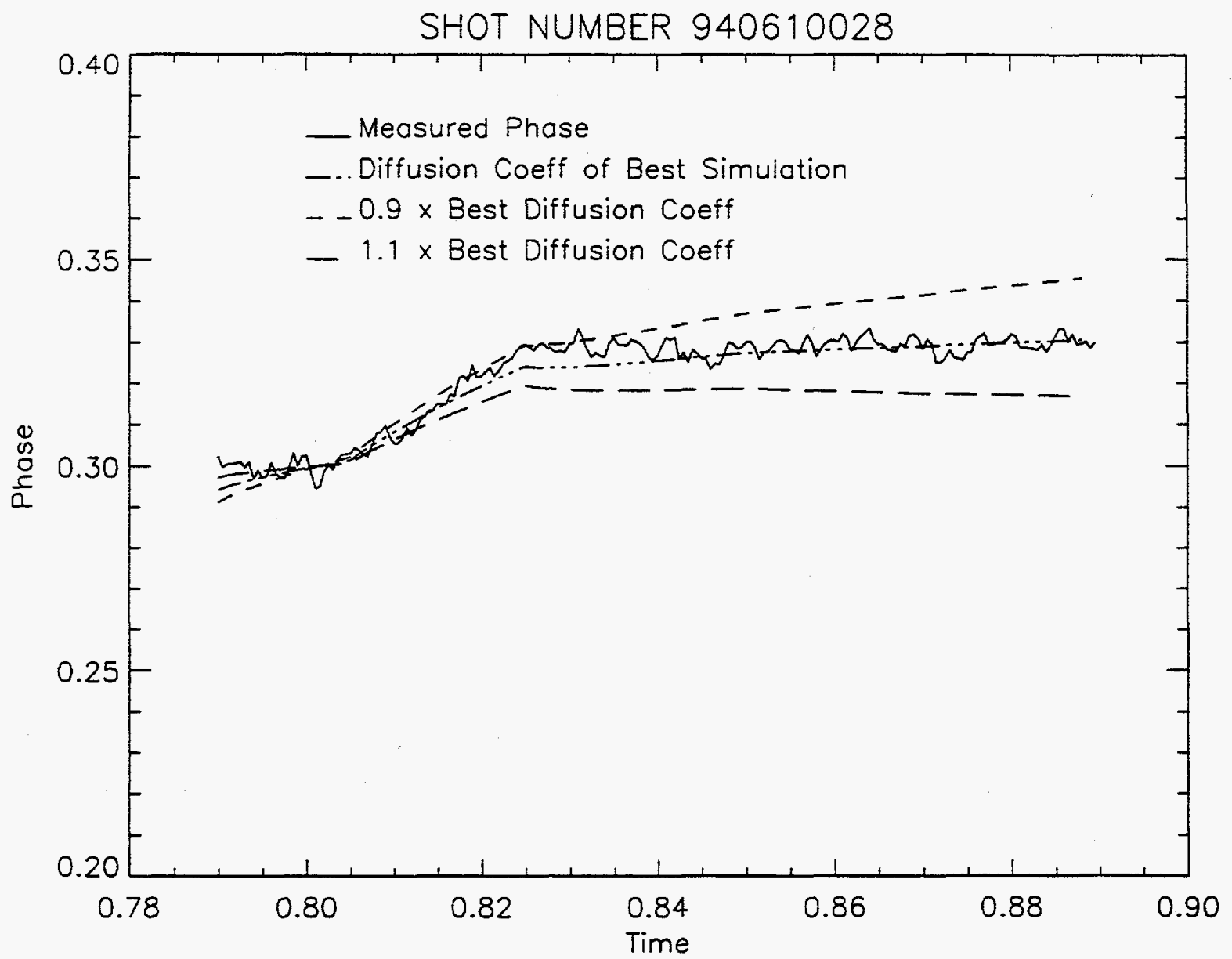

Figure 6.21: Variation of the simulated phase signals when the transport coefficients are varied by $\pm 10 \%$ while maintaining the $V / D$ ratio. 


\section{Chapter 7}

\section{Interpretation of Results}

In this chapter, a consistent explanation for the central density rise after

detachment will be presented. Comparison of results with other measurements on Alcator C-Mod will be made as well as comparisons to particle transport measurements made on other tokamaks.

\subsection{Interpretation of Core Particle Measurements on C-Mod}

The magnitude of the transport coefficients measured on Alcator C-Mod are consistent with measurements made by other experiments. Experimentally measured values of $D(r)$ and $V(r)$ fall in the range of $0.005<D<1.0 \mathrm{~m}^{2} / \mathrm{s}$ and $0<V<10.0 \mathrm{~m} / \mathrm{s}$. The values for Alcator C-Mod fall well within these boundaries. Furthermore, the predicted neoclassical values have been calculated at $\Psi$ equal to 0.5 using:

$$
\begin{aligned}
& D_{P S}=q^{2} v_{e i} \rho_{L}{ }^{2}, \\
& D_{\text {Banana }}=\varepsilon^{-\frac{3}{2}} q^{2} v_{e i} \rho_{L}{ }^{2}, \\
& V_{\text {Ware }}=\varepsilon^{\frac{1}{2}} \frac{E_{\text {parallel }}}{B_{P}},
\end{aligned}
$$

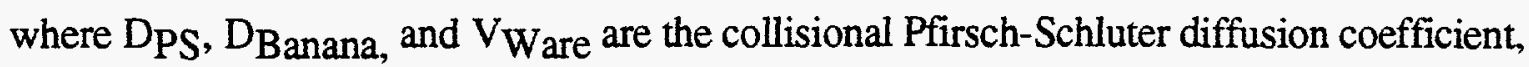
the Banana regime diffusion coefficient and the Ware pinch velocity, respectively [124, 125]. The variables are: $q$, the safety factor; $\rho_{L}$, the Lamour radius; $v_{e i}$, the electron-ion collision frequency, $\varepsilon$ the ratio the minor and major radii; $E_{\text {parallel, the parallel electric }}$ 
field; and $\mathrm{B}_{\mathrm{p}}$, the poloidal magnetic field. The predicted values were at least a factor of 2 smaller than the measured profiles for all except the highest density. At $1.3 \times 10^{20} \mathrm{~m}^{-3}$, the measured and predicted values were comparable. The value of the Ware pinch was anomalously small for all cases. The results are summarized in Table 7.1. They are in agreement with results from PLT, T-10, MTX, and TFTR which observed measured transport coefficients that were up to 100 times larger than neoclassical predictions [74, $104,108,113]$.

\begin{tabular}{|c|c|c|c|c|c|c|}
\hline Density & Temp & $\begin{array}{c}\text { Measured } \\
\text { Diffusion } \\
\text { Coefficient }\end{array}$ & $\begin{array}{c}\text { Pfirsch- } \\
\text { Schluter } \\
\text { Diffusion }\end{array}$ & $\begin{array}{c}\text { Banana } \\
\text { Regime } \\
\text { Diffusion }\end{array}$ & $\begin{array}{c}\text { Measured } \\
\text { Pinch } \\
\text { Velocity }\end{array}$ & $\begin{array}{c}\text { Ware } \\
\text { Pinch }\end{array}$ \\
\hline $\mathrm{m}^{-3}$ & $\mathrm{keV}$ & $\mathrm{m}^{2} / \mathrm{s}$ & $\mathrm{m}^{2} / \mathrm{s}$ & $\mathrm{m}^{2} / \mathrm{s}$ & $\mathrm{m} / \mathrm{s}$ & $\mathrm{m} / \mathrm{s}$ \\
\hline $8.68 \times 10^{19}$ & 1.05 & 0.23 & .003 & .054 & 1.5 & 0.16 \\
\hline $1.02 \times 10^{20}$ & 0.89 & 0.17 & .004 & .068 & 0.95 & 0.16 \\
\hline $1.13 \times 10^{20}$ & 0.90 & 0.14 & .004 & .076 & 0.78 & 0.16 \\
\hline $1.22 \times 10^{19}$ & 0.80 & 0.06 & .005 & .087 & 0.25 & 0.16 \\
\hline
\end{tabular}

Table 7.1 Comparison of Measured and Neoclassical transport coefficients at $\Psi=0.5$.

From the results of chapter 6 , the transport shows a dependence on the background density. In figure 6.10 , the profiles of the coefficients decrease as $\bar{n}_{e}$ increases. The coefficients at $\Psi$ equal to 0.5 of the gas modulated shots are plotted as a function of $1 / \bar{n}_{e}$ in figure 7.1. The points agree well with an inverse scaling for both $\mathrm{V}$ and D. This dependence with density is similar to the scaling found at TFTR, T-10, Text, and ASDEX $[104,105,106,108]$. At ASDEX Gentle found that $D$ and $V$ both decrease by an order of magnitude as the density increases by a factor of 5 . However, this decrease saturates for the saturated Ohmic confinement mode (SOC) at higher densities. In the 

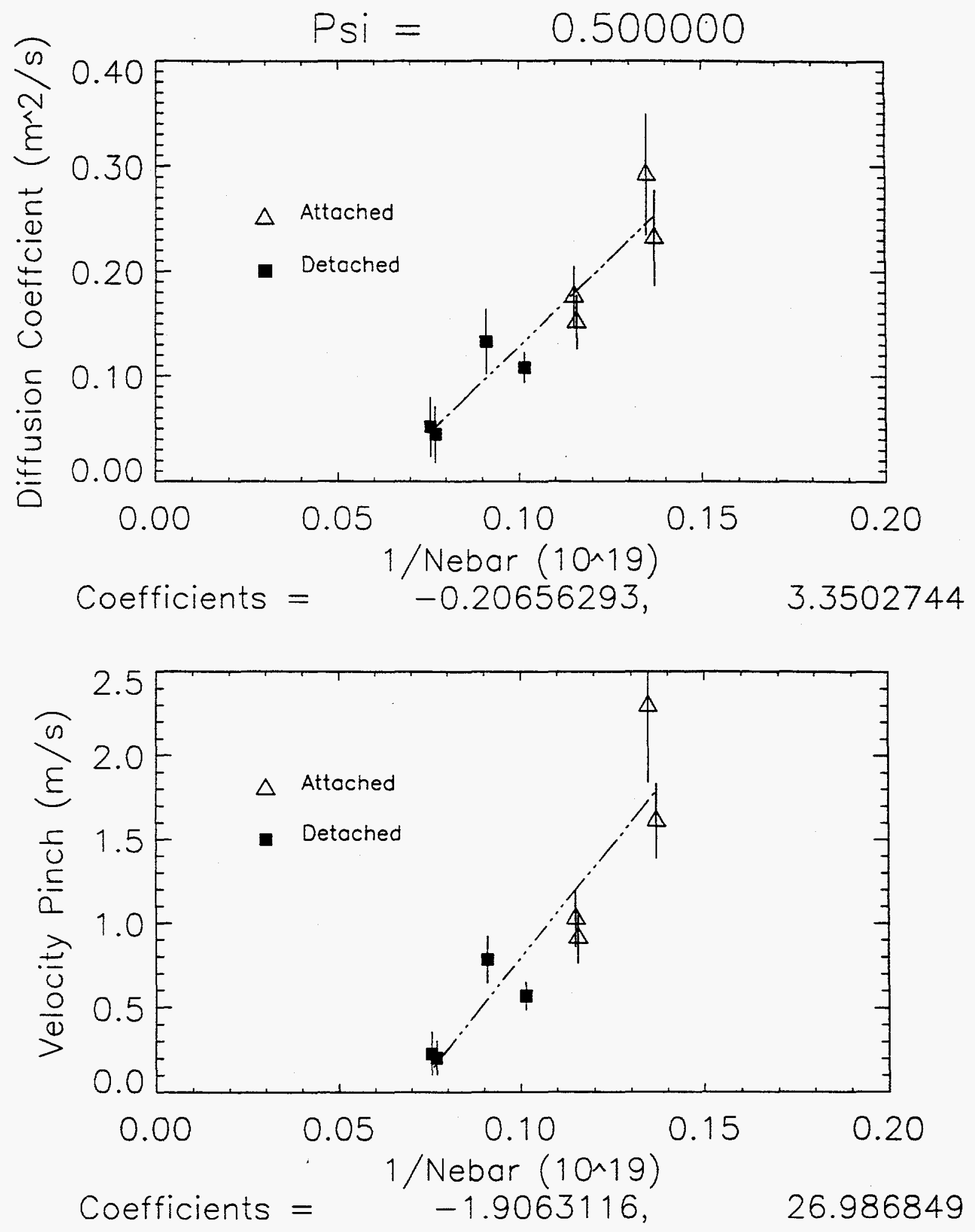

Figure 7.1 Scaling of transport coefficients at $\Psi=0.5$ with the inverse density 
improved Ohmic confinement mode (IOC), the decrease in the coefficients continues with rising density and approaches a factor of 4 at the higher densities. Both particle and energy confinement exhibit this behavior and this reduction in the diffusion coefficient was large enough as to result in an observed density rise even without gas fueling [106]. At TEXT, a clear dependence on $1 / \bar{n}_{e}$ was measured for fixed $\mathrm{B}_{\mathrm{T}}$ and Ip [105]. Similarly, Efthimion observed that the profile averaged diffusion coefficients scaled as $1 / \mathrm{n}_{\mathrm{e}}$ on TFTR. At MTX, experiments showed that the diffusion coefficients increased with density but the measurements were done near the density limit where the plasma behavior may be quite different [74].

While the density scaling is consistent with previous experiments, it is of greater note that both detached and attached shots follow the same scaling. This would indicate that the rise in central density is not due to a change in the fundamental transport. The detached divertor is not a new confinement mode. Otherwise, there should be a discontinuity in the transport scaling when the plasma detaches. The reduction in the transport coefficients is consistent with a rise in density and no anomalous changes can be seen in the core transport.

\subsection{Energy Confinement}

Measurements of the energy confinement time, $\tau_{\mathrm{e}}$, have been made by Greenwald for the gas modulated shots [114]. Estimates of the stored energy were made by using diamagnetic loop measurements and the calculation of the kinetic energy from temperature and density measurements. The estimates from each method were in good agreement and the measured confinement times are given in Table 7.2. 


\begin{tabular}{|c|c|c|}
\hline Shot & $\begin{array}{c}\text { Attached or } \\
\text { Detached }\end{array}$ & $\begin{array}{c}\text { Confinement } \\
\text { Time (ms) }\end{array}$ \\
\hline 940610015 & A & $35 \pm 5$ \\
\hline 940610016 & A & $31 \pm 3$ \\
\hline 940610017 & A & $28 \pm 5$ \\
\hline 940610023 & D & $30 \pm 5$ \\
\hline 940610024 & D & $33 \pm 3$ \\
\hline 940610025 & D & $30 \pm 3$ \\
\hline
\end{tabular}

Table 7.2 Energy Confinement time for Gas Modulated Shots [114].

The energy confinement time exhibits negligible change when the plasma detaches. This is consistent with the hypothesis that detachment doesn't represent a new confinement mode. If there is no change in the underlying particle transport, the energy confinement would be expected to remain constant also. The confinement times are consistent with L-Mode scaling which exhibits very weak dependence on density.

\subsection{Impurity Transport}

As described in chapter 2, Alcator C-Mod has an operating laser ablation system that can be used for the study of impurity transport. By ablating small quantities of impurities into the plasma, the impurity transport can be followed by the VUV spectrometer, soft $\mathrm{x}$-ray array and $\mathrm{x}$-ray spectrometer. The measured brightness and emissivities can be used to determine the time history of the density profiles for the different charge states. Figure 7.2 gives an example of the decay of the line emission radiation from scandium which was ablated at $550 \mathrm{~ms}$. The MIST transport code is then used to model the decay time to determine the transport coefficients [62]. Typical measured values for the diffusion coefficients are given in Table 7.3. 

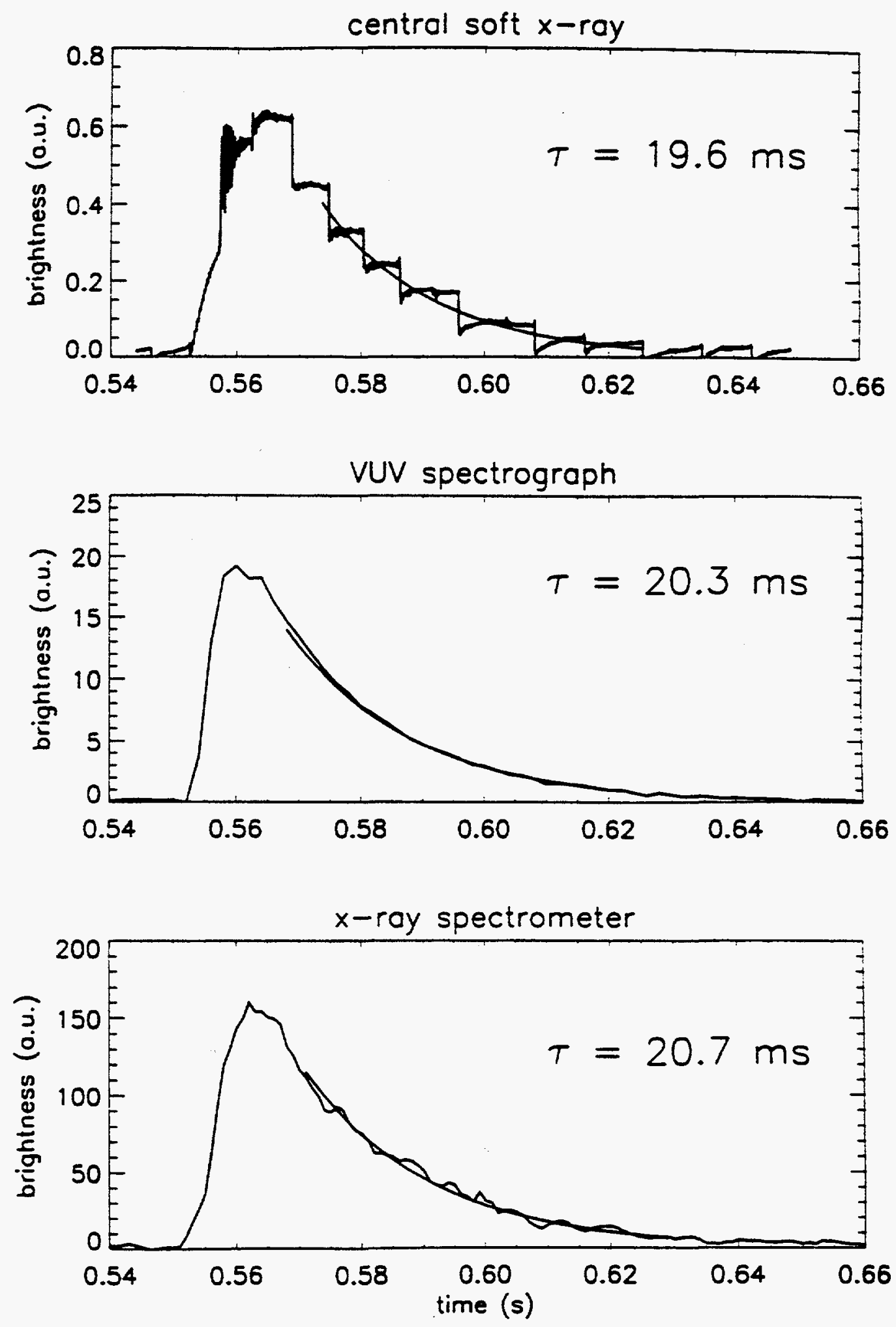

Figure 7.2: Time history of the brightness of the Scandium line emission as measured by $\mathrm{x}$-ray and VUV diagnostics. Scandium ablation was at $550 \mathrm{~ms}$. 


\begin{tabular}{|c|c|c|}
\hline $\begin{array}{c}\text { Plasma Current } \\
(\mathrm{kA})\end{array}$ & $\begin{array}{c}\text { Diffusion } \\
\text { Coefficient }\left(\mathrm{m}^{2} / \mathrm{s}\right)\end{array}$ & $\begin{array}{c}\text { Pinch Velocity } \\
(\mathrm{m} / \mathrm{s})\end{array}$ \\
\hline 600 & 0.65 & 3.25 \\
\hline 800 & 0.5 & 2.5 \\
\hline
\end{tabular}

Table 7.3: Impurity transport coefficients measured by the laser ablation technique [115]

Measurements also show that there is no noticeable change in the impurity transport when a divertor detachment occurs. Again, this supports the hypothesis that the underlying transport doesn't change because it would be inconsistent for only particle transport to be improved without seeing similar changes in the impurity and energy transport.

\subsection{Improvement in Fueling Efficiency}

The experimental results presented here imply that there is no change in the particle transport that would account for the rise in central density during detachment. An alternative explanation for detachment is that the source term changes. Measurements of the argon density in the core by the $\mathrm{x}$-ray spectrometer indicate that during detachment the argon density increases. This is illustrated in figure 7.3 where the argon density rises quickly after the detachment at $600 \mathrm{~ms}$. The characteristic drop in divertor probe signal and the rise in electron density are also shown.

If there is no change in the particle/impurity transport, the increase in argon is due to a change in the source; either the source has increased in magnitude or the screening of argon neutrals is diminished and the argon is being ionized deeper in the plasma. The main source of argon in Alcator C-Mod is by injection from a piezo-electric valve. It is a controlled source so it is very unlikely that the actual source size has increased. However, a decrease in the effectiveness of the plasma shielding is possible. It has been observed 


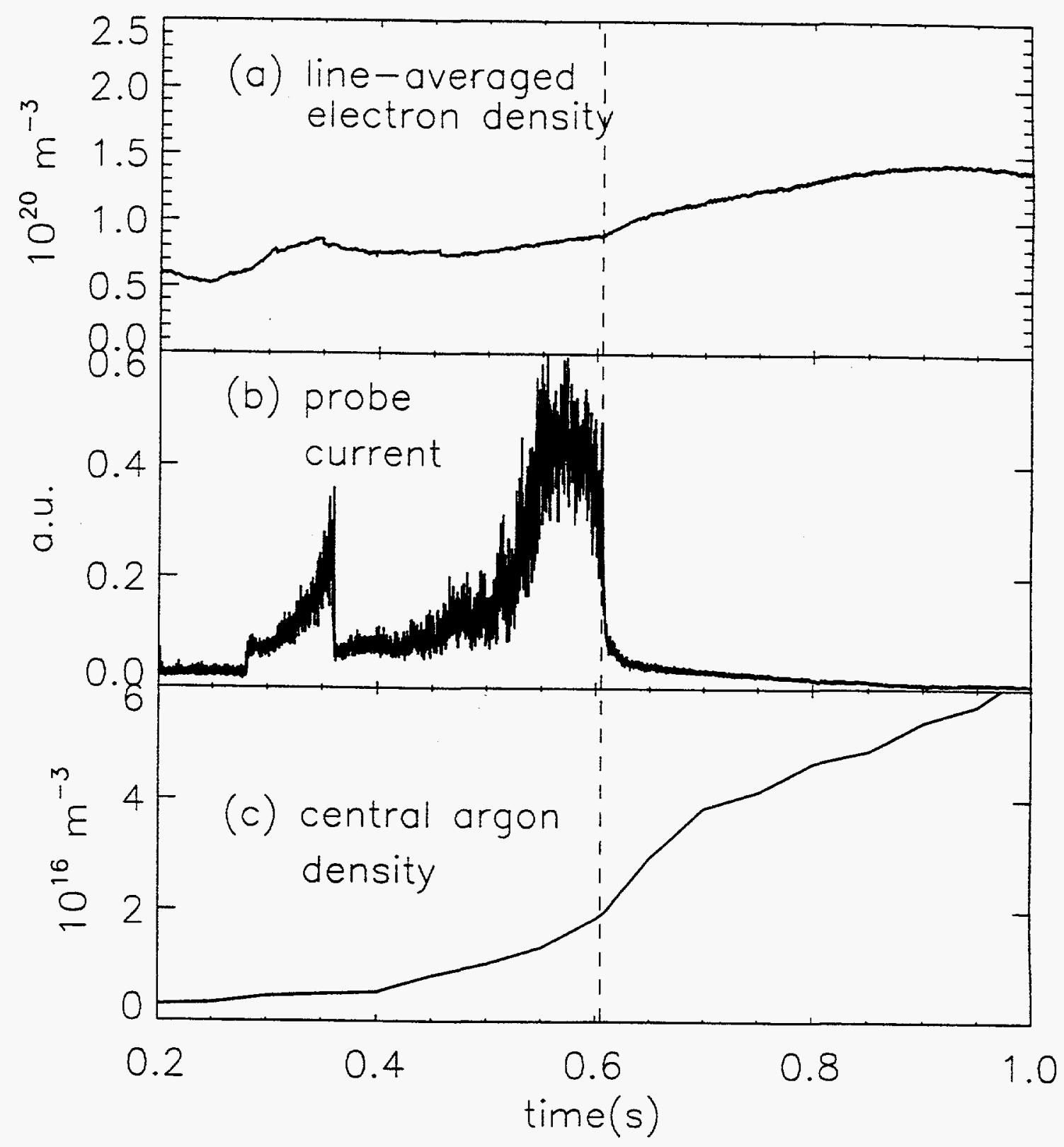

Figure 7.3: Rise in the central Argon density after detachment. 
that for attached, ohmic plasmas, the penetration of impurities into the core decreases with increasing plasma density. This is due to a reduction in the mean free path of impurity neutrals in the scrapeoff layer. Similarly, a possible explanation for the increase in argon after detachment is that the mean free path of the impurity neutrals increases after detachment due to a drop in the temperature of the scrapeoff layer. The temperature during detachment is below $5 \mathrm{eV}$ at the divertor plates. Thus, impurity neutrals penetrate further into the plasma before being ionized. This would increase the likelihood that the impurity atoms would be transported to the core of the plasma and result in the higher core argon density.

\subsection{Measurements from the $H_{\alpha}$ Tomography}

Measurements of the $H_{\alpha}$ radiation have been made during divertor detachment. A tomographic inversion technique has been developed by Kurz to provide detailed information about the spatial distribution of the $\mathrm{H}_{\alpha}$ radiation [116]. Figures 7.4 and 7.5 illustrates the distribution of radiation before and after a divertor detachment. The radiation source has moved closer to the $\mathrm{x}$-point and inside the separatrix after the detachment. This implies that the electron source has moved deeper into the plasma and is consistent with the concept that the central density rises due to a change in the source term. By folding in the density and temperature profiles from the ECE and TCI diagnostics, it is possible to calculate the number of ionizations inside the LCFS. Figure 7.6 shows that at the time of detachment, the source term within the LCFS increases significantly. This agrees well with estimates of the source size from the simulation. Simulations were run for profiles before and after detachment. An equilibrium source and transport coefficients were determined before detachment. Using the same transport coefficients, a new source size was then determined for after detachment. This new source was estimated to be a factor of 2 larger than the pre-detachment source. 


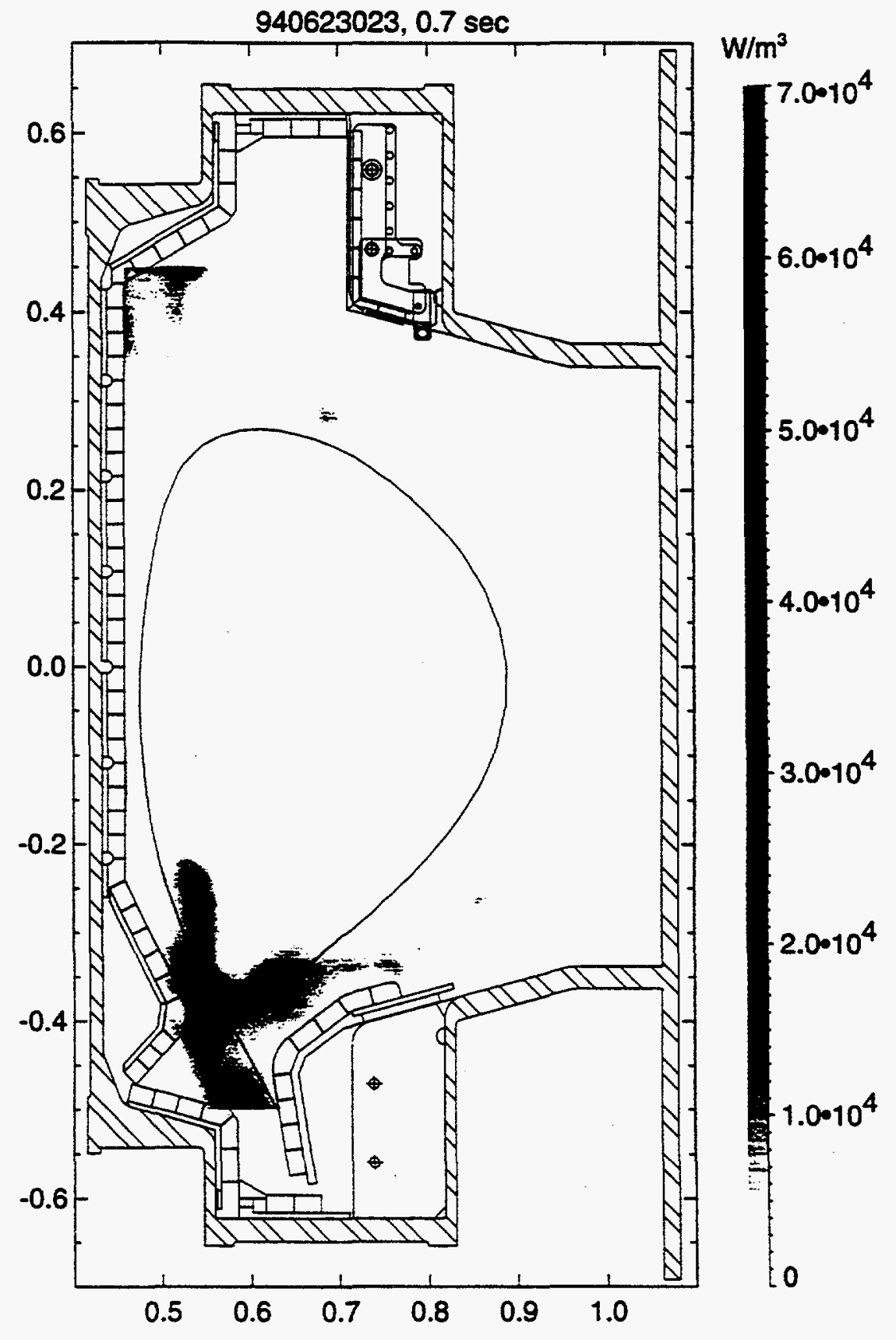

Figure 7.4: Spatial distribution of the $\mathrm{H} \alpha$ radiation before detachment at $800 \mathrm{~ms}$. 


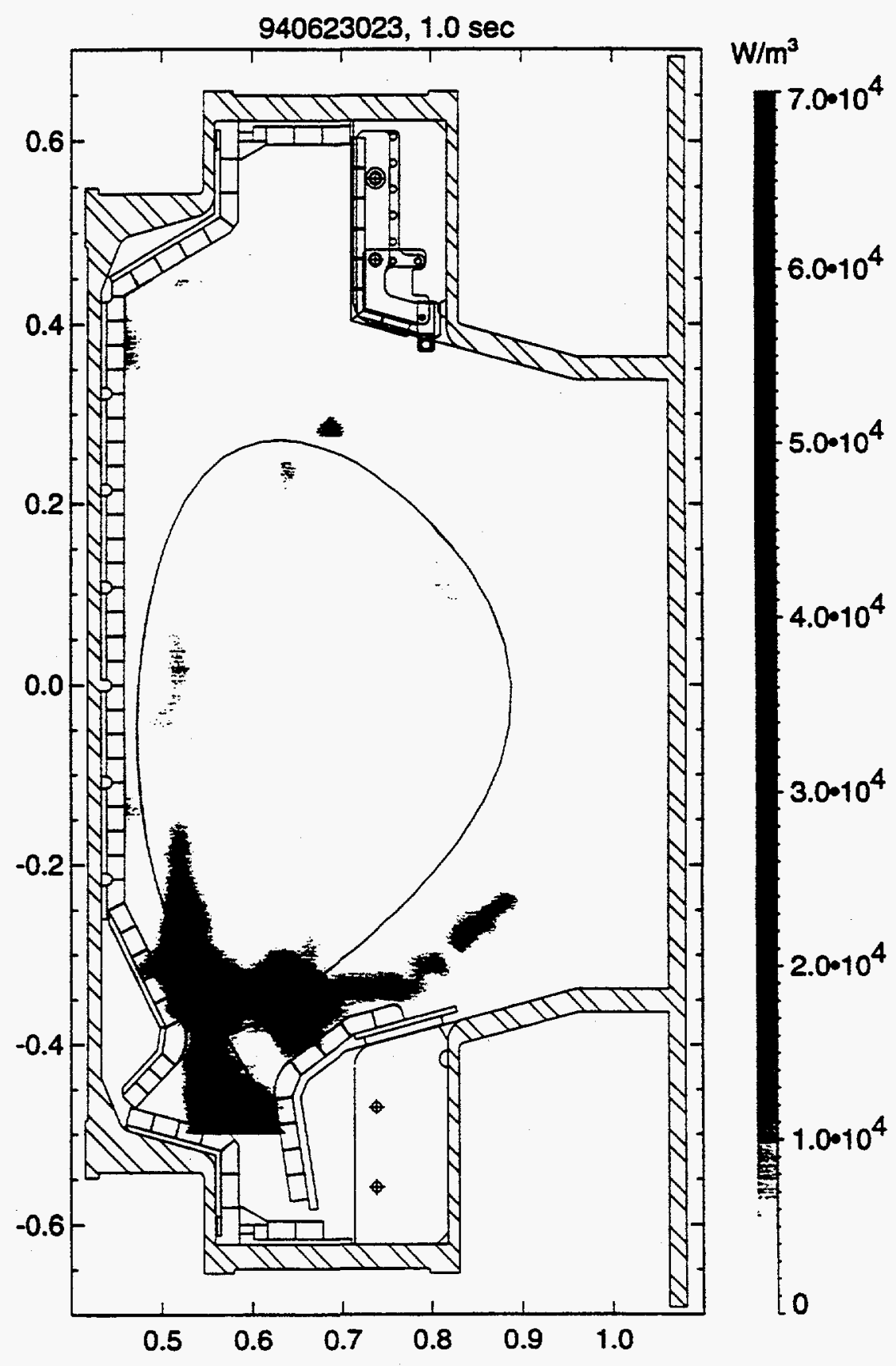

Figure 7.5: Spatial distribution of the $\mathrm{H} \alpha$ radiation after detachment at $800 \mathrm{~ms}$. The source increases and moves inside the LCFS. 


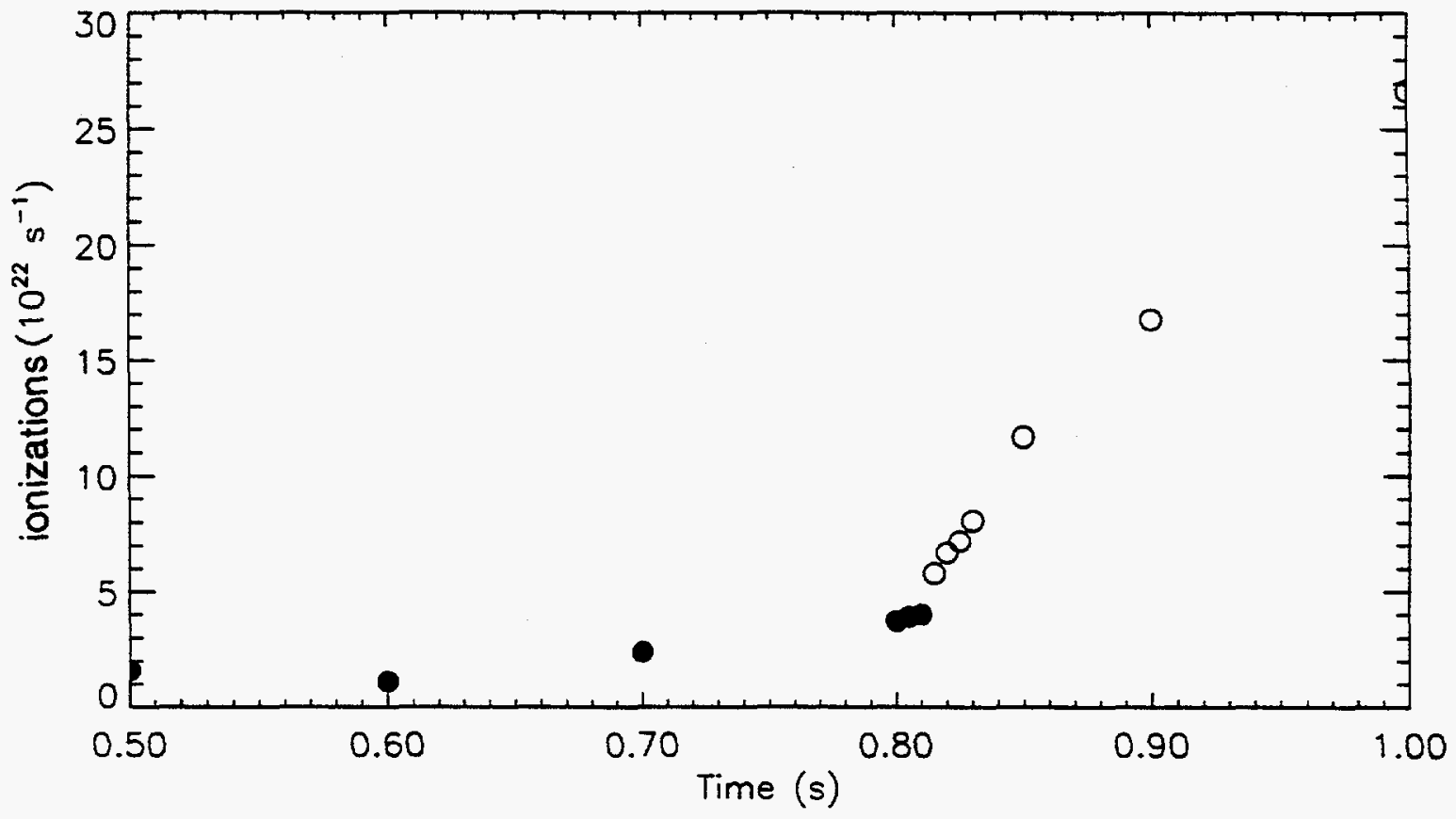

Figure 7.6: Number of ionizations inside the LCFS. Detachment occurs at $800 \mathrm{~ms}$. 


\subsection{Hypothesis for the Rise in Central Density After Detachment}

The rise in central electron density is not due to a change in the fundamental processes governing the core transport of Alcator C-Mod. Measurements of the transport coefficients indicate that both attached and detached plasmas follow the same scaling with the underlying plasma density. Furthermore, there are no indications of changes in the energy or impurity transport after detachment. If the density rise were due to a transition to a new confinement mode with different transport mechanisms, changes in the transport of all three quantities would be expected.

A more consistent explanation for the density increase is a change in the electron source term. Measurements of the Argon transport show that the mean free path of neutral Ar increases after detachment. An analogous argument can be made for hydrogen or deuterium neutrals. Measurement of the $\mathrm{H}_{\alpha}$ radiation indicate that the source term moves deeper inside the plasma. The size of the total source also increases which is consistent with simulation results that estimate that there is an increase in the source by a factor of 2. The number of ionizations has clearly increased within the LCFS. However, it is not possible to differentiate between an increase of the ionizations due to a larger overall source or a decrease in plasma screening of the neutrals. The temperature in the scrapeoff layer has decreased significantly which would increase the mean free path but this is balanced by the effect of a higher edge density. 


\section{Chapter 8}

\section{Conclusion}

\subsection{Summary of Experimental Results}

There is a great need to develop the ability to control the plasma density and the shape of the density profile. Alcator C-Mod, with its high field, high density operating regime and its set of high temporal and spatial resolution diagnostics offers a unique opportunity to conduct particle transport studies. To perform these dedicated studies, a two color interferometer has been constructed to provide reliable measurements of the particle density with $2 \mathrm{~cm}$ spatial resolution and $1.0 \mu \mathrm{s} \mathrm{temporal} \mathrm{resolution.} \mathrm{It} \mathrm{is} \mathrm{capable}$ of following many different plasma phenomena ranging from simple parabolic profiles to double pellet injection and snakes.

An inversion technique based on the SVD matrix inversion methodology is applied to the line integrated measurements to provide density profiles. A detailed analysis has been developed to determine an edge density consistent with reflectometer, divertor probe and limiter probe measurements. Sensitivity studies show that the largest source of error for the inversion is from the estimation of the edge density. The effect of the edge gradient and magnetic geometry are secondary effects.

During the operation of Alcator C-Mod, a phenomena known as divertor detachment was observed. During the detachment, the particle and energy flux to the divertor plates dropped dramatically. The central electron density exhibited a sudden rise by as much as $50 \%$. To investigate this phenomena, a series of gas modulation shots were performed at a constant magnetic field of $5.3 \mathrm{~T}$ and a plasma current of $630 \mathrm{kA}$. The line

averaged electron density was varied between $7.0 \times 10^{19}-1.4 \times 10^{20} \mathrm{~m}^{-3}$. Care was taken to keep the perturbation levels below $10 \%$ and to maintain the stability of the 
plasma. The perturbed diffusion coefficients were extracted through a time regression analysis and also a numerical simulation analysis. The profiles of the transport coefficients are in good agreement from both analyses as can be seen in fig. 6.18. Again sensitivity studies of the regression analysis show that the greatest source of error in this analysis is the value of the edge density. For different edge densities, there is sufficient change in the inverted profiles that the regressed transport coefficients exhibit a decrease with the edge density. The regression was performed with the inversion using the best estimate of the edge density from several measurements. Again, other sources of errors such as the smoothing weight and magnetic geometry were negligible.

The profiles of $\mathrm{V}$ and $\mathrm{D}$ increase toward the plasma edge. Measured values of the diffusion coefficients are consistent with those found in other particle transport experiments and are a factor of 10 above neoclassical predictions [104,105,106, 108]. The most significant result is the inverse dependence of the transport coefficients on the plasma density. From fig 7.1 , both the attached and detached shots follow a $1 / \bar{n}_{e}$ scaling which is well documented by other experiments. This density effect would not be seen in either the energy transport which follows L-mode scaling and has little density dependence or in the impurity transport which exhibits a weak correlation with the plasma density. The scaling is indicative that the underlying particle transport remains unchanged after detachment and that the detached plasma does not represent a new enhanced confinement mode.

However, measurements of the $\mathrm{H}_{\alpha}$ radiation indicate that the number of ionizations inside the last closed flux surface increases significantly. This is consistent with results from the numerical transport simulation which estimate an increase of 2 in the source size. Measurements show that Ar density increases in the core after detachment. This is explained by a decrease in the plasma shielding and greater penetration of $\mathrm{Ar}$ neutrals inside the LCFS. Thus, the experimental results indicate that the rise in the 
central density is consistent with an increase in the electron source after detachment and not a change in the underlying transport processes.

\subsection{Future Plans}

The current series of gas modulation experiments has been able to rule out the detached plasmas as a new confinement mode. Fortunately, there are many other possible transport studies to be done on Alcator C-Mod. A natural extension would be a study of the dependence of the transport coefficients on plasma current. The effect of H-Mode and ICRF heating on the transport coefficients can also be examined. Finally, the study of pellet ablation and density effects such as snakes can be accomplished. The addition of the reflectometer and the Thomson Scattering measurements will greatly facilitate the determination of the edge density and help the inversion of the density profiles. The gas modulation technique has proven to be a useful and valuable tool for particle transport studies and the groundwork laid by this thesis will provide a good foundation for fruitful results in the future. 


\section{Appendix A}

\section{Numerical Simulation}

This appendix will provide the details concerning the numerical simulation analysis. The basic idea behind the analysis is to simulate the time evolution of the density profile and, given the magnetic geometry, to calculate the expected phase shifts on the interferometer from the time history of the density. These computed phase shifts can be compared to actual measurements. The diffusion coefficients can then be adjusted until there is a match. The methodology has been described in section 5.6. The equation that is being simulated is the particle continuity equation given by eq. 5.1 ,

$$
\frac{d g}{d t}+\frac{1}{\operatorname{Vol}^{\prime}(\Psi)} \frac{d\left(\operatorname{Vol}^{\prime}(\Psi) F\right)}{d \Psi}=S
$$

Here, $\mathrm{g}$ is the quantity of interest, Vol is the volume of the flux surface, $\mathrm{F}$ is the flux associated with $\mathrm{g}, \mathrm{S}$ is the source term and $\Psi$ is the flux variable. This equation can be generally reduced by making the assumption of $\mathrm{g}$ being a flux quantity and integrating over the poloidal variable. For the electron density, eq. A.1 can be reduced to the more familiar equation,

$$
\frac{d n}{d t}=-\nabla \cdot \Gamma+S
$$

The time derivative of the electron density, $\mathrm{n}$, is equal to the divergence of the particle flux, $\Gamma$, plus the source term, $S$. In the simulation, the flux is composed of a diffusive term and a convective term, 


$$
\Gamma=-D \nabla n-n V
$$

A simple finite differencing code was developed to model this time evolution. The change in density on a flux surface is given by,

$$
\Delta n_{j}=\frac{\Delta t}{V o l_{j}} \cdot\left(F_{j} A_{j}-F_{j-1} A_{j-1}\right)
$$

$A_{j}$ is the surface area of the $j^{\text {th }}$ flux surface, $\Delta t$ is the time step of the simulation, Vol $j$ is the plasma volume between the $j^{\text {th }}$ and $j-1$ th flux surfaces and $F_{j}$ is the particle flux at the $j^{\text {th }}$ surface which can be written as,

$$
F_{j}=D_{j} \frac{n_{j+1}-n_{j-1}}{2 \Delta r}+n_{j} V_{j}
$$

$D_{j}$ is the diffusion coefficient at $j, V_{j}$ is the pinch velocity, and $\Delta r$ is the spatial step. Care must be taken to correctly define the location of the flux surfaces and the locations where the density is defined. Furthermore, the temporal and spatial stepsize must be adjusted to prevent instabilities in the time evolution.

The source term is a Gaussian function centered at the minor radius and with a width of $1 \mathrm{~cm}$. Sensitivity studies were done varying the width of the source from $1 \mathrm{~cm}$ to $5 \mathrm{~cm}$. For the lowest density case, in which the source term would have the greatest impact, Fig. A.1 illustrates the simulated profiles for source widths of 1 and $5 \mathrm{~cm}$. There was a negligible effect in the simulated transport coefficient profiles due to the source width. In addition, the neutral density profile was calculated with the FRANTIC code using density and temperature profiles with a peak density of $7.5 \times 10^{19} \mathrm{~m}-3$ and a peak temperature of $1.8 \mathrm{keV} \mathrm{[126].} \mathrm{This} \mathrm{scenario} \mathrm{represents} \mathrm{a} \mathrm{low} \mathrm{density,} \mathrm{high} \mathrm{temperature}$ 
case in which the neutrals should have the largest penetration. From Fig. A.2, the neutral density falls a factor of 10 in the first $2 \mathrm{~cm}$. This validates the use of a $1 \mathrm{~cm}$ width in the numerical simulation. For most of the core plasma the source term is negligible when the fueling is localized at the edge. Thus the source term is given by

$$
S(r)=S_{0} e^{\frac{-(r-a)^{2}}{2 w_{0}}} .
$$

$\mathrm{S}_{0}$ is the source size, $\mathrm{w}_{0}$ is the source width and $\mathrm{a}$ is the minor radius.

The final consideration is the boundary conditions of the simulation. The first derivative of the density profile is set to zero by keeping the first two density points equal. Also, a point outside the minor radius is set to zero to act as a sink of electrons. Additional details of numerical simulations are given by Vasins $[87,111,120]$. 

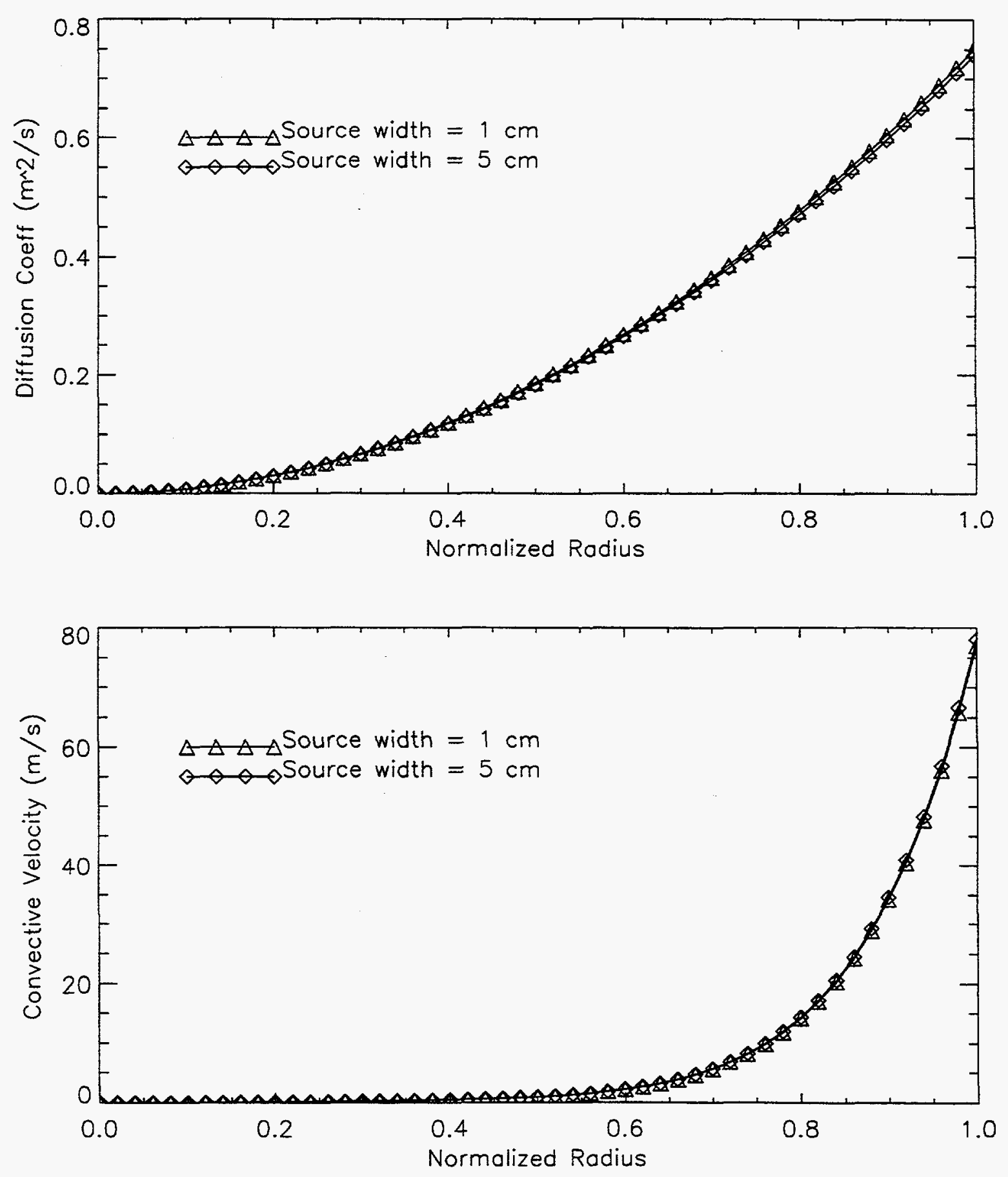

Figure A.1: Sensitivity of simulated transport coefficient profiles with source width. 


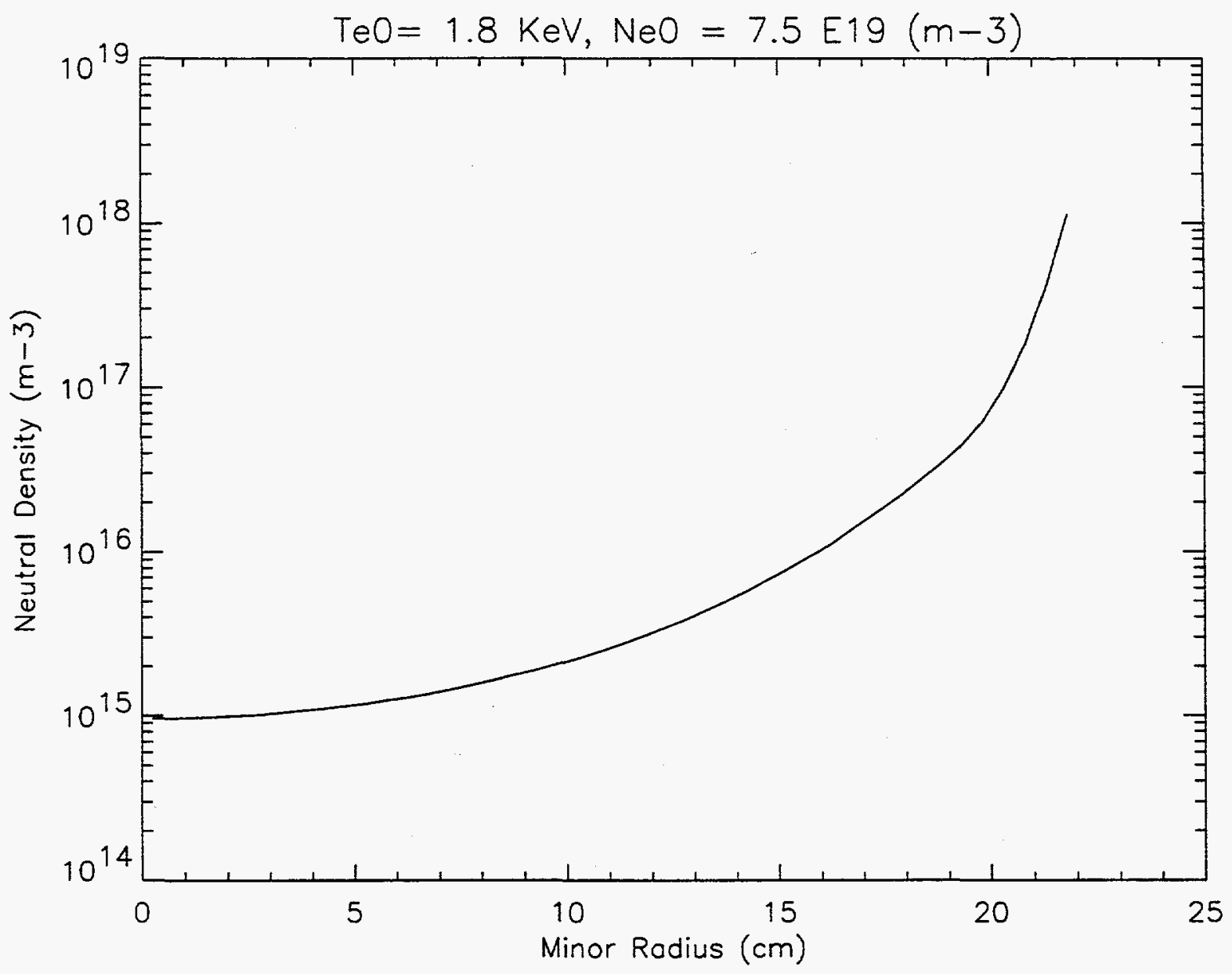

Figure A.2: Neutral density profile from FRANTIC code. 


\section{References}

[1] J. D. Callen, Phys. Fluids B2, 2869 (1990).

[2] J. Hugill, Nucl. Fusion 23, 331 (1983).

[3] S. I. Braginskii, in Review of Plasma Physics, edited by M. A. Leontovich, (Consultants Bureau, NewYork, 1965)l, Vol. 1, p. 205.

[4] K. H. Burrell et al., Phys. Fluids B2, 2904 (1990).

[5] R.J. Groebner, W. Pfeiffer, F. P. Blau, K. H. Burrell, E. S. Fairbanks, R. P. Seraydarian, H. St. John, and R.E. Stockdale, Nucl. Fusion 26, 543 (1985)

[6] R.J. Fonck, R. Howell, K. Jaehnig, L. Roquemore, G. Schilling, S. Scott, M. C. Zarnsdorff, C. Bush, R. Goldston, H. Hsuan, D. Johnson, A. Ramsey, J. Schivell, and H. Towner, Phys. Rev Lett. 63, 520 (1989).

[7] S. M. Kaye and R.J Goldston, Nucl Fusion 25, 65 (1985).

[8] R. Parker, M. Greenwald, S. Luckhardt, E. Marmar, M. Porkolab, and S. Wolfe, Nucl. Fusion 25, 1127 (1985).

[9] R. D. Stambaugh, Phys. Fluids B2, 2941 (1990).

[10] F. Wagner et al., Phys. Rev. Lett. 49, 1408 (1982).

[11] F. Soldner, E. R. Muller, F. Wagner, and H. S. Bosch, Phys. Rev. Lett. 61, 1105 (1988).

[12] T. Watari et al., Nucl. Fusion 30, 1197 (1990).

[13] M. J. Greenwald et al., Phys. Rev. Lett. 53, 352 (1984).

[14] M. Kauffman et al., Nucl. Fusion 28, 827 (1988).

[15] M. G. Bell and the TFTR Group, in Plasma Physics and Controlled Nuclear Fusion Research 1988, Nice (IAEA, Vienna 1989). Vol I. p. 27.

[16] K.H. Burrell, Plasma Phys. Controlled Fusion 31, 1649 (1989).

[17] J. D. Strachan et al., Phys. Rev. Lett. 58, 1004 (1987). 
[18] B. Coppi, M. N. Rosenbluth, and R. Z. Sagdeev, Phys. Fluids 10, 582 (1967).

[19] W. Horton, D. Choi, and W. M. Tang, Phys. Fluids 24, 1077 (1981).

[20] P. Guzdar et al., Phys. Fluids 29, 3291 (1986).

[21] R. J Fonck et al., in Proceeding of the Fourth Symposium on Heating in Toroidal Plasmas (ENEA, Rome, 1984), Vol. 1, P. 37.

[22] ASDEX Team, Nucl. Fusion 29, 1959 (1989).

[23] C.E. Bush et al., Bull. Amm. Phys. Soc. 34, 2012 (1989).

[24] M. Keilhacker and the JET Team, in Plasma Physics and Controlled Nuclear Fusion Research 1988, Nice ( IAEA, Vienna, 1989), Vol. I, p. 159.

[25] K. McGuire et al., Plasma Physics and Controlled Nuclear Fusion Research 1984, London ( IAEA, Vienna, 1985), Vol. I, p. 117.

[26] A. Tanga et al., Plasma Physics and Controlled Nuclear Fusion Research 1986, Kyoto ( IAEA, Vienna, 1987), Vol. I, p. 65.

[27] M. Matsumoto, A. Fumakoshi, M. Hasewawa, K. Hoshino, and S. Kasai, in Proceedings of Fourteenth European Conference on Controlled Fusion and Plasma Physics, Madrid (EPS, Petit-Lancy, Switzerland, 1987), Vol. I, p. 5.

[28] K. Steingmetz et al., Phys. Rev. Lett. 58, 124 (1987).

[29] T.H. Osborne et al., Nucl. Fusion 30, 2023 (1990).

[30] M. Keilhacker et al., in Plasma Physics and Controlled Nuclear Fusion Research 1984, London ( IAEA, Vienna, 1985), Vol. I, p. 71.

[31] M. A. Mahdavi, in Proceedings of Sixteenth European Conference on Controlled Fusion and Plasma Physics, Venice (EPS, Petit-Lancy, Switzerland, 1989), p. 249.

[32] O. Gruber et al., in Proceedings of Twelfth European Conference on Controlled Fusion and Plasma Physics, Budapest (EPS, Petit-Lancy, Switzerland, 1985), Part. I, p. 18.

[33] R. Childs, J. Goetz, M. Graf, A. Hubbard, J. Rice and T. Toland, Fifteenth IEEE Nuclear and Plasma Science Symposium on Fusion Eng., Hyannis (1993).

[34] N. Ohyabu, G. L. Jahns, R. D. Stambaugh, and E. J . Strait, Phys. Rev. Lett. 58, 20 (1987). 
[35] S. M. Kaye et al., J. Nucl. Mater. 121, 115 (1984).

[36] G. Becker, ASDEX-Team, and Neutral Injection Team, Nucl. Fusion 22, 1589 (1982).

[37] M. Watkins, Plasma Phys. Controlled Fusion 31, 1713 (1989).

[38] F. Wagner et al., Phys. Rev. Lett. 54, 1453 (1984)

[39] M. J. Greenwald et al., in Plasma Physics and Controlled Nuclear Fusion Research 1984, London (IAEA, Vienna, 1985), Vol. I, p. 45.

[40] S. M. Wolfe et al., Nucl. Fusion 26, 329 (1986).

[41] M. Kaufmann et al., in Plasma Physics and Controlled Nuclear Fusion Research 1988, Nice (IAEA, Vienna, 1989), Vol. I, p. 229.

[42] D. P. Schissel et al., Phys. Fluids B1, 1843 (1989)

[43] J. M. Noterdaeme, in Plasma Physics and Controlled Nuclear Fusion Research 1988, Nice ( IAEA, Vienna, 1989), Vol. I, p. 583.

[44] R. D. Petrasso, D. J. Sigmar, K. W. Wenzel, and J.E. Hopf, Phys. Rev. Lett. 57, 707 (1986)

[45] K. Kawahata et al., in Plasma Physics and Controlled Nuclear Fusion Research 1988, Nice ( IAEA, Vienna, 1989), Vol. I, p. 287.

[46] D. L. Brower et al., Phys. Rev. Lett. 59, 48 (1987).

[47] P. C. Stangeby, and G. M. McCracken, Nucl. Fusion 30, 1225 (1990).

[48] M. Zarnstorff et al., in Proceedings of Fifteenth European Conference on Controlled Fusion and Plasma Physics, (EPS, Petit-Lancy, Switzerland, 1985), Vol. 12B, Part. I, p. 95 .

[49] R.J. Fonck, R. Howell, K. Jaehnig, L. Roquemore, Phys. Rev. Lett. 63, 520 (1989).

[50] R.J. Groebner et al., Nucl. Fusion 26, 543 (1986)

[51] M. Zarnstorff et al., in Plasma Physics and Controlled Nuclear Fusion Research 1988, Nice ( IAEA, Vienna, 1989), Vol. I, p. 183. 
[52] T.W. Petrie et al., Proceeding of the Tenth International Conference on PlasmaSurface Interactions on Controlled Fusion Devices, Journal of Nuclear Materials, 848 (1992).

[53] G. Janeschitz et al., Proceedings from the Nineteenth European Conference on Controlled Fusion and Plasma Physics, Innsbruck (EPS, Petit-Lancy, Switzerland, 1985), Vol. 16C, Part. II, p. 727.

[54] I. H. Hutchinson et al., Phys. Plasmas 1, 1511 (1994)

[55] B. Labombard, B. Lipshultz, and D. Jablonski, Bull. Am. Phys. Soc.38 (1993)

[56] E. Apgar, B. Coppi, A. Gondhalekkar, H. Helava, D. Komm et al., in Plasma Physics and Controlled Fusion Research 1976, Berchtesgaden (IAEA, Vienna, 1977), Vol I, p. 247.

[57] M. Gaudreau, A Gondalekar, M. H. Hughes, D. Overskei, and D .S. Pappas, Phys. Rev. Lett. 39, 199 (1977)

[58] Alcator Group, The Physics and Engineering of Alcator C-Mod, MIT Plasma Fusion Center Report PFC/RR-88-11, (1988)

[59] P. C. Stek, J. H. Irby, LAE Technical Meeting on Microwave Reflectometry for Fusion Plasma Diagnostics, 1994.

[60] B. Lipshultz et al., Proc. Eleventh Int. Conf. on Plasma-Surface Interactions, Mito, Japan, 1994 ( to be published).

[61] E. S. Marmar, J. L .Cecchi, S. A. Cohen, Rev. of Scientific Instruments, 46, 1149 (1975).

[62] M. A. Graf, J. E. Rice, J. L. Terry, et al., Rev. of Scientific Instrument, 1994 ( to be published)

[63] A. Gondhalekkar, R. S. Granetz, et al., in Plasma Physics and Controlled Fusion Research 1978, Innsbruuck ( IAEA, Vienna, 1979), Vol I, p. 199.

[64] PLT Group, in Plasma Physics and Controlled Fusion Research 1978, Innsbruuck ( IAEA, Vienna, 1979), Vol I, p. 167.

[65] C.S. Chang, F. L. Hinton, Phys. Fluids 25, 1493 (1982)

[66] Alcator Group, Alcator C Status and Program Plan, MIT Plasma Fusion Center Report PFC/RR-82-03, (1982) 
[67] S. Golovato et al., in Proc. Twentieth Eur. Conf. on Controlled Fusion and Plasma Physics, Montpelier 1994, (1994). (to be published)

[68] M. Greenwald et al.,Proc. Fifteenth Int. Conf. on Plasma Physics and Controlled Nuclear Fusion Research., Seville 1994 ( to be published).

[69] J. A. Snipes et al, First Ohmic H-Modes in Alcator C-Mod, MIT Plasma Fusion Center Report PFC/JA-94-02, (1994)

[70] D. Veron, in Infrared and Millimeter Waves, K. J. Buuton ed., Vol. 2. New York: Academic Press, (1979).

[71] I. H. Hutchinson, in Principles of Plasma Diagnostics, Cambridge: Cambridge University Press, (1987).

[72] B. W. Rice, Thesis, Poloidal Magnetic Field Measurements on the Microwave Tokamak Experiment Using Far-Infrared Interferometry, University of California at Davis (1992).

[73] J.. H Irby, E. S. Marmar, E. Sevillano, and S. M. Wolfe, Rev. Sci. Instrum. 59, 1568 (1988)

[74] M. M. Marinak, Thesis, Behaviour of the Particle Transport Coefficient Near the Density Limit in MTX, University of California at Berkeley (1993)

[75] S. K. Kim et al., Rev. Sci. Instrum. 59, 1551 (1988)

[76] G. Braithwaite et al., Rev. Sci. Instrum. 60, 2825 (1989)

[77] T. N. Carlstrom, D. R. Ahlgren, and J. Crosbie, Rev. Sci. Instrum. 59, 1063 (1988).

[78] T. Luke, J. H. Irby, Bull. Am. Phys. Soc.35 (1990)

[79] T. Luke, J. H. Irby, Bull. Am. Phys. Soc.34 (1989)

[80] P. Innocente and S. Martini, Rev. Sci. Instrum. 59, 1571 (1988)

[81] G. Gorini, and N. Gottardi, Jet Report, JET-R(90)03, (1990)

[82] N. Gottardi, J. Appl. Phys. 50, 2647 (1979).

[83] L. R. Baylor et al., Oak Ridge National Laboratory, ORNL/TM-11758, (1991)

[84] Y. Yasutomo et al. IEEE Trans. Plasma Sci. PS-9, 18 (1981) 
[85] H. K. Park, Plasma Phys Controlled Fusion 31, 2035 (1989)

[86] H. K. Park, Rev. Sci. Instrum. 61, 2879 (1990).

[87]W. H. Press, B. P. Flannery, L. A. Teukolsky, W. T. Vetterling, Numerical Recipes: The Art of Scientific Computing, Cambridge University Press, (1986).

[88] L. L. Lao, H. St. John, R. D. Stambaugh, A. G. Kellman, and W. Pfeiffer, NuCl. Fusion 25, 1611 (1985)

[89] B. Labombard, D. Jablonski, B. Lipshultz, G. McCracken, J. Goetz, Eleventh Int. Conf. on Plasma-Surface Interactions, Mito, Japan, 1994 ( to be published).

[90] B. Labombard, private communication.

[91] B. Coppi, and N. Sharky, Nucl. Fusion 21, 1363 (1981).

[92] E. D. Fredrickson et al., Nucl. Fusion 26, 849 (1986).

[93] J. D. Callen, J. P. Christiansen, J .G. Cordey, P. R. Thomas, and K. Thomsen, Nucl. Fusion 27, 1857 (1987).

[94] K. W. Gentle, Phys. Fluids 31, 1105 (1988).

[95] N. J Cardozo et al., Plasma Phys. Controlled Fusion 32, 983 (1990).

[96] J. D. Callen and G. L .Jahns, Phys. Rev. Lett. 38, 491 (1977).

[97] S. K. Kim, D. L. Brower, W. A. Peebles and N. C. Luhmann Jr., Phys. Rev. Lett. 60, 577 (1988).

[98] A. C. C. Sips, A.. E. Costley, J. C. M. Haas, and R. Prentice, in Proceedings of the Sixteenth European Conference on Controlled Fusion and Plasma Physics (EPS, PetitLancy, Switzerland, 1989, Vol.1, p. 99.

[99] A. Cheetham et al., in Plasma Physics and Controlled Nuclear Fusion Research 1988, Nice ( IAEA, Vienna, 1989).

[100] C. C. Gomez, Thesis, Massachusetts Institute of Technology, (1986).

[101] L. R. Baylor et al., Nucl. Fusion 31, 1249 (1991).

[102] R. A. Hulse et al., in Proc. Fourteenth Eur. Conf. on Controlled Fusion and Plasma Phys. 1987, Madrid, Vol. 11D, Part 1, p. 318. 
[103] M. Greenwald et al., in Plasma Physics and Controlled Nuclear Fusion Research 1986, Kyoto (IAEA, Vienna, 1987), Vol. I, p. 139.

[104] A. A. Bagdasarov, N. L. Vasin et al., in Plasma Physics and Controlled Nuclear Fusion Research 1984, London ( IAEA, Vienna, 1985), Vol. I, p. 181.

[105] K. W. Gentle, B. Richards, and F. Waelbroeck, Plasma Phys Controlled Fusion 29, 1077 (1987)

[106] K. W. Gentle, O. Gehre, and K. Kreiger, Nucl. Fusion 32, 217 (1992).

[107] K. Nagashima, A. Sakasai, and T. Fukada, Nucl. Fusion 33, 1677 (1993).

[108] P. C. Efthimion et al.,in Plasma Physics and Controlled Nuclear Fusion Research 1988, Nice ( IAEA, Vienna, 1989), Vol. I, p. 307.

[109] D. K. Mansfield et al., in Proc. Fourteenth Eur. Conf. on Controlled Fusion and Plasma Phys. 1987, Madrid, Vol. 11D, Part 1, p. 314.

[110] F. Galton, Proc. Royal Society, London, Vol. 40, (1886).

[111] N. L. Vasins, V. A. Vershkov, V. A. Zhuravlev, Sov. J. Plasma Physics 10, 525 (1984)

[112] B. Lipshultz, B. Labombard, E. S. Marmar et al., Nucl. Fusion 24, 977 (1984).

[113] J. D. Strachan et al., Nucl. Fusion 22, 1145 (1982)

[114] M. Greenwald, private communication.

[115] M. Graf, private communication.

[116] C. Kurz, B. Lipshultz, G. McCracken, M. Graf, J. Snipes, J. Terry and B. Welsh, Eleventh Int. Conf. on Plasma-Surface Interactions, Mito, Japan, 1994 ( to be published).

[117] J. Irby, private communication.

[118] Y. Takase, private communication.

[119] J. Goetz, private communication.

[120] B. Lipshultz, Plasma Fusion Center, Quarterly Review, (1993).

[121] K. Nagashima and A. Sakasai, Rep. JAERI-M 92-057, JAERI, Ibaraki (1992). 
[122] R. J. Hawryluk, 'An Empirical approach to Tokamak Transport'. in Physics of Plasmas Close to Thermonuclear Conditions, ed. by B. Coppi, et al., (CEC, Brussels, 1980), Vol. 1, p. 19.

[123] JET Team,in Plasma Physics and Controlled Nuclear Fusion Research 1988, Nice (IAEA, Vienna, 1989), Vol. I, p. 215.

[124] J. Wesson, Tokamaks, (Oxford University Press, New York, 1987), p. 94.

[125] F. L. Hinton and R. D. Hazeltine, Rev. Mod. Phys. 48, 230 (1976).

[126] S. Tamour, SAI Report \# 023-79-10J6LJ, (1979). 